\title{
A straightforward and Lagrangian proof of the Einsteinian equivalence between the mass and the inernal energy (i.e. rest energy) V2
}

Özgür Berké, Portet-sur-Garonne, ozgur.berke@live.fr

I propose a Lagrangian proof of Einstein's well-known law that the mass of any system is its internal energy. The interest of this proof is to show how the distinction between internal degrees of freedom and the center of mass appears in the Lagrangian formalism. Considering that the Lagrangian depends on a particular set of variables for the internal degree of freedom, I show in a standard Lagrangian way how one can naturally find the desired law. This proof does not use the tensors of energy-momentum and can be easily used by students familiar with Lagrangian mechanics and the basis of Special Relativity. I apply the method for the particles and for the field, using the scalar field for simplification but it is easy to generalize for other fields (containing only the first derivative in Lagrangian). I give the example for the gravitation field. The method permits us to observe a strong relation between the Einstein's $E=m c^{2}$ law and his other famous law of the time dilation. I carefully analyze the meaning of the particular choice of the variable and showing a sort of a modified speed addition formula without contradicting, of course, the one of Einstein (\& Poincaré). I also try to untangle, with this point of view, the relation between the mass and the origin of the energy scale. Finally I analyze the reason why in Newtonian mechanic we don't have a such law. In the annex I give some elements of the Hamiltonian analysis checking again the coherence of the particular set of variables and I apply this way of thinking to the old Lorentz-Poincaré model of the electron (useful for an explicit classical renormalization of the mass).

Keywords: Special Relativity, $\mathrm{E}=\mathrm{mc}^{2}$, center of mass, Internal Energy, Lagrangian, Lorentz Transformation

I declare that:

- there was no funding used for this article;

- there is no conflict of interest;

- there is no competing interest;

Acknowledgement : To my wife 


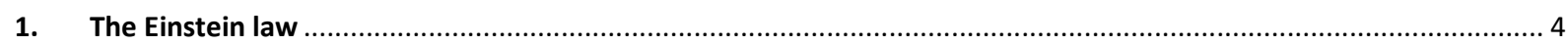

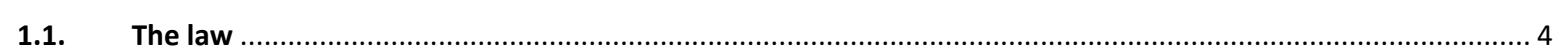

1.2. The current proof (I work in the Landau-Lifchitz notation Books [1],[2]) ............................................ 6

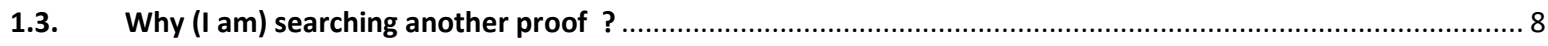

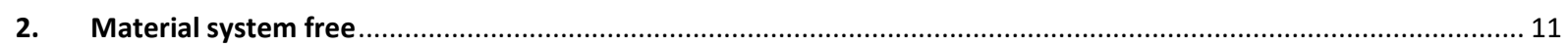

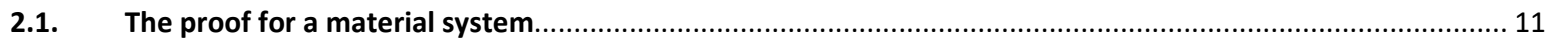

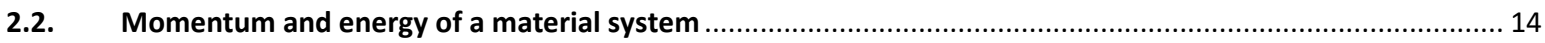

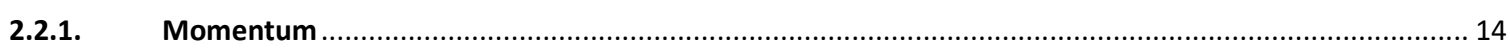

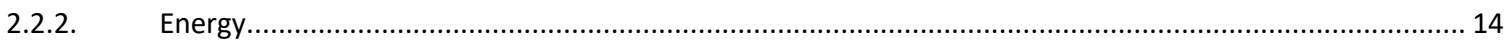

2.3. The Euler-Lagrange equation for the internal particles and the mass center .......................................... 15

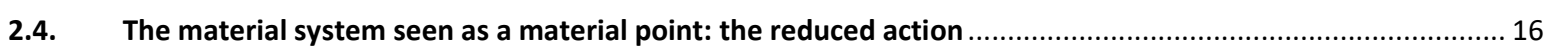

2.5. The material system seen as a material point: the internal dynamic is known .......................................... 18

2.6. A strong link between the Einstein law and the dilation of time .......................................................... 20

2.7. Questions about the meaning of events and physical quantities used in the proof ...................................... 24

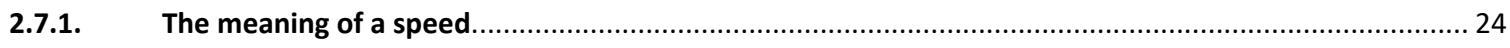

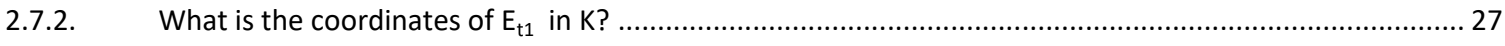

2.7.3. What is the difference of coordinates of the particle for infinitesimal interval $\mathrm{dt}$, seen in $\mathrm{K}$ ? .................. 30

2.7.4. What is the difference of coordinates of the particle for infinitesimal interval $\mathrm{dt}$, seen in $\mathrm{K}^{*} \ldots \ldots \ldots \ldots \ldots \ldots . . . . . . . . .32$

2.7.5. What is the expression of the speed in $\mathrm{K}$ and $\mathrm{K}^{*}$ and what are their relation (velocity addition formula)? 35

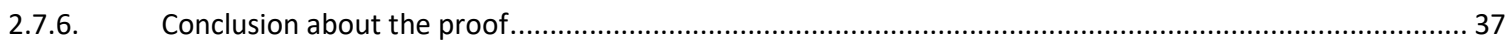

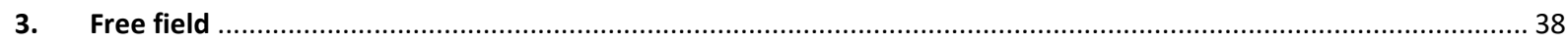

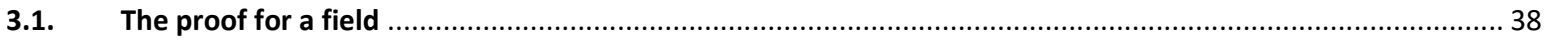

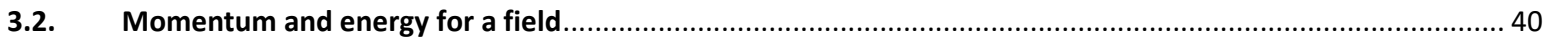

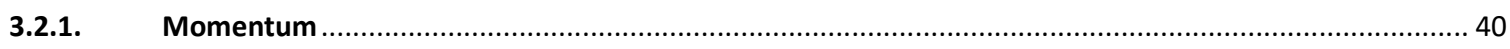

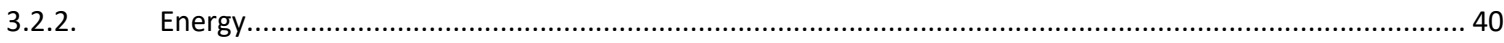

3.3. The Euler-Lagrange equation for the internal field and the mass center ................................................. 41

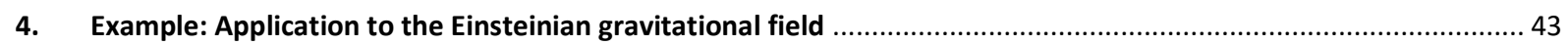

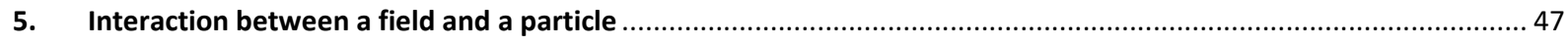

6. Does the mass of a body depend on the indeterminacy of the origin of energy? ............................................... 48

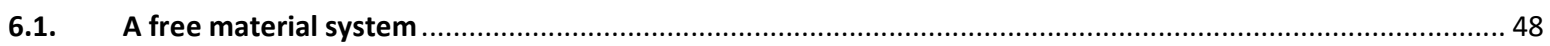

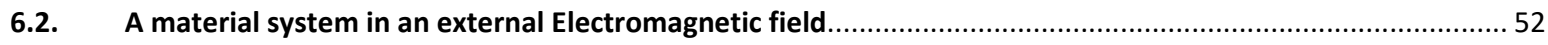

6.3. A material system in a non-Minkowskian space-time (General Relativity) .............................................. 57

7. Why the Einstein law (the mass as internal energy) does not appear in Newtonian mechanics? The crucial role of

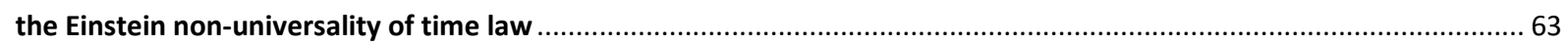

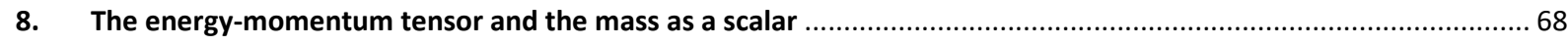

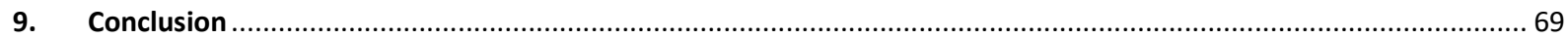

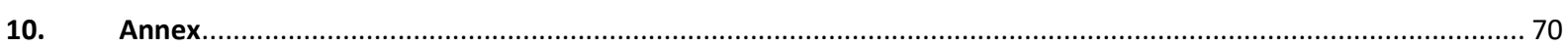

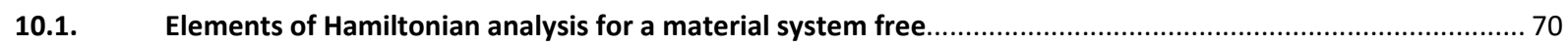

10.2. Application: the electromagnetic model of the electron [3], [9 ], [23]-[25] .......................................... 75 
10.3. Hamiltonian analysis: Hamilton-Jacobi equation(an attempt) for a material system free

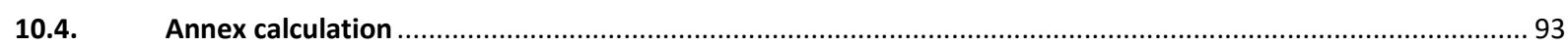

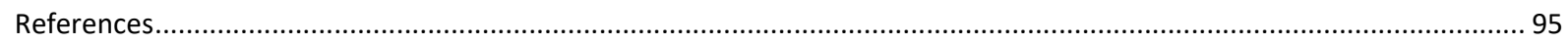




\section{The Einstein law}

\subsection{The law}

According the expression of the law of physics via the principle of least action [1] and the relativistic invariance: the mass $m_{a}$ of a material point " $a$ " is simply the multiplicative coefficient appearing in the Lagrangian of this material point, interacting or not with an external field.

$$
S\left[\boldsymbol{r}_{\boldsymbol{a}}(t)\right]=-\int_{s_{a, 1}}^{s_{a, 2}} m_{a} \cdot c \cdot d s_{a}+\cdots=-\int_{t_{1}}^{t_{2}} \frac{m_{a} \cdot c^{2}}{\gamma\left(\boldsymbol{v}_{\boldsymbol{a}}\right)} d t+\cdots
$$

In 1905, Einstein tells us that whatever the system: a set of material points (dynamically characterised with a Lagrangian $L\left(\left\{\boldsymbol{r}_{\boldsymbol{a}}\right\},\left\{\frac{d \boldsymbol{r}_{\boldsymbol{a}}}{d t}\right\}\right)$ ) or a field (dynamically characterised with the Lagrangian $\left.\Lambda\left(\varphi, \frac{\partial \varphi}{\partial r}, \frac{\partial \varphi}{\partial t}\right)\right)$ we should have:

$$
S\left[\boldsymbol{R}_{\boldsymbol{c}}(t), \ldots\right]=-\int_{t_{1}}^{t_{2}} \frac{E^{*}}{\gamma\left(\boldsymbol{V}_{\boldsymbol{c}}\right)} d t+\cdots
$$

$\circ$ With $E^{*}=\sum_{a} \frac{d r_{a}^{*}}{d t^{*}} \frac{\partial L^{*}}{\partial \frac{d r_{a}^{*}}{d t^{*}}}-L^{*}\left(\left\{\boldsymbol{r}_{\boldsymbol{a}}^{*}\right\},\left\{\frac{d \boldsymbol{r}_{a}^{*}}{d t^{*}}\right\}\right)$ for a material system;

$\circ \quad$ Or $E^{*} \equiv \iiint\left(\frac{\partial \varphi^{*}}{\partial t^{*}} \frac{\partial}{\partial\left(\frac{\partial \varphi^{*}}{\partial t^{*}}\right)} \Lambda^{*}-\Lambda^{*}\right) d V^{*}$ for a scalar field (for example).

Where the quantities with a star * are relative to the reference frame associated to the mass center $\mathrm{K}^{*}$. So $E^{*}$ is the internal energy.

Thus, every system has a centre of mass which has a Lagrangian, analogous to a material point with a mass $M=\frac{E^{*}}{c^{2}}$. This is the famous law of Einstein.

The possibility to highlight the existence of a such theoretical result, emerging from the dynamics of Special Relativity, greatly impress its discoverer: this law "has an extraordinary theoretical impact" (Einstein in [17]) in physics. Indeed:

- The sole fundamental, a priori invariable, characteristic, the mass, of the only object in Newtonian Mechanical Theory, the material point, is suggested to be an effective dynamical description of a variable quantity, the internal energy. The latter could be related to:

- a collection of material points;

$\circ$ and rather a more fundamental object of other kind.

- The first possibility, the most conservative one, gives rise to the astonishing idea that

- every energy (if and only if one can see it at rest) could constituted a part of a mass;

- every energy (without restriction) could be transformed to a mass by the common transformation of this energy in an internal energy, this is always possible via the energetic channels surfing on the (Lagrangian) coupling (directly or indirectly) between all kind of system: kinetic energy, heat, electromagnetic... ;

(this is why $E^{*}=M c^{2}$ is abusively [12] changing into a " $E=M c^{2 "}$ or noted more correctly $\Delta E=\Delta M c^{2}$, the latter law have to be carefully understand as conjunction of 2 laws:

- the possibility to transform every energy variation $\Delta E$ in a part of an internal energy $E^{* \prime}=E^{*}+$ $\Delta E$, calling an absorption

- $\quad$ and the true Einstein law: $E^{*}=M c^{2}$ 
- $\quad \Rightarrow \Delta E=E^{*^{\prime}}-E^{*}=M^{\prime} c^{2}-M c^{2}=\Delta M c^{2}$

- The second possibility is I think the final logical step to the application of the Einstein law: as every material point could be always suspected to be an effective description, the only other object which as far as we know can replace it is a field. Then the Einstein law demands/suggests in fact the substitution of the material points by fields: we slide slowly from one object to another, Special Relativity suggests itself to be a (Special-)Relativistic Fields Theory ;

(But, because of the experimental stability of the mass (the existence of some irreducibility values), in contrast to the variability of a field, this (Special-)Relativistic Fields Theory necessitates therefore another stability principle in order to be experimentally relevant: this new (or combination of) principle(s) is(are) actually playing by the Quantum (Special-Relativistic) Field Theory). 


\subsection{The current proof (I work in the Landau-Lifchitz notation Books [1],[2])}

This law was clearly formulated as a general law of physic since its first conceptual-invention and publication in 1905 by Einstein. The future famous physicist has established his new law in a very convincing way but as an explorer did, combining rigorous mathematic with (as often in a first breakthrough) an a priori implicit (and intuitively) well motivated hypotheses, but necessary restricted to a certain context (see [10] for an example of a posterior critical review). After this first breakthrough the law was re-demonstrated more clearly by Einstein himself in a different ways (cf. [12] and the piece of Einstein articles [14]\&[15]-[21]), and also with the aid of other physicist like Von Laue, Felix Klein (cf. [11]) in order, as it should for honest and thus perfectionists physicists, to reassuring themselves by searching to face against any possible objection which could coming in their mind (their own judge) about the general validity of the law. The most general proof (that the author know and read firstly in [1], in conjunction with [3]), is to demonstrate that the momentum is a 4-vector.

Indeed, tanks to the stress energy tensor $T^{i k}$ of the system, we can always ([1]) associate to it a 4vector $P^{i}\left(K^{*}\right) \equiv \frac{1}{c} \iiint \int_{\text {space-time,K }} T^{i k} d S_{k}$, where we choose the hyper-surface of integration as the hyperplane of the reference frame $\mathrm{K}^{*}\left(t^{*}=c t e\right)$.

In any frame ([3]), $P^{i}\left(K^{*}\right)$ can be written equivalently

$$
P^{i}\left(K^{*}\right)=\frac{1}{c} \iiint \int_{\text {space-time }} T^{i k} \delta\left(n_{l m} x^{l} \eta^{m}\left(K^{*}\right)\right) \cdot \eta_{k}\left(K^{*}\right) d^{4} x
$$

where $\eta_{k}\left(K^{*}\right)$ is an orthogonal vector of the hyperplane $t^{*}=$ cte of $\mathrm{K}^{*}$ such that $\eta^{*}{ }_{k}\left(K^{*}\right)=(1,0,0,0)$ in $K^{*}$.

Thus, the Lorentz transformations tells us:

$$
P^{i}\left(K^{*}\right)=\frac{1}{c} \iiint \int_{\text {space-tim }} L_{r}^{i} L_{s}^{k} T^{* r s} \delta\left(t^{*}\right) \cdot L_{k}^{m} \cdot \eta_{m}^{*}\left(K^{*}\right) d^{4} x^{*}=L_{r}^{i} \frac{1}{c} \iiint_{x^{* \alpha} \in V^{*}} T^{* r 0}\left(0, x^{* \alpha}\right) d V^{*}
$$

So $P^{i}\left(K^{*}\right)=L_{r}^{i} P^{* r}\left(K^{*}\right)$ where $P^{* r}\left(K^{*}\right)=\frac{1}{c} \iiint_{\text {Space }} T^{* r 0}\left(0, x^{* \alpha}\right) d V^{*}$

But $E^{*} \equiv \iiint_{\text {Space }} T^{* 00}\left(0, x^{* \alpha}\right) d V^{*}$ and $P^{* \alpha}\left(K^{*}\right) \equiv 0$ by definition of $\mathrm{K}^{*}$

So we have $P^{i}\left(K^{*}\right)=\left(\gamma \frac{E^{*}}{c}, \gamma \frac{E^{*}}{c^{2}} \boldsymbol{V}_{\mathbf{K} * / \mathbf{K}}\right)$, hence $\boldsymbol{P}=\gamma \frac{E^{*}}{c^{2}} \boldsymbol{V}_{\mathbf{K} * / \mathbf{K}}=>M=\frac{E^{*}}{c^{2}}$

That is to say, the 3-momentum of any system is the same as a material point:

○ with a mass $M=\frac{E^{*}}{c^{2}}$;

$\circ \quad$ and a speed $\boldsymbol{v}=\boldsymbol{V}_{\mathbf{K} * / \mathbf{K}}$.

2 remarks:

○ $P^{i}\left(K^{*}\right)$ is here relative to the particular time $t^{*}=0$ and is not a priori constant; 
- $P^{i}\left(K^{*}\right)$ is not the only one 4-momentum since we can define a different one for each frame of reference, $P^{i}(K), P^{i}\left(K^{\prime}\right), P^{i}\left(K^{*}\right) \ldots$, all are associated to different hyperplanes of simultaneity linked to each possible (an infinity) frame of reference $K, K^{\prime}, K^{*} \ldots$ (see [3]).

It exists a particular case where there is only one 4-momentum $P^{i}: P^{i}(K)=P^{i}\left(K^{\prime}\right)=P^{i}\left(K^{*}\right) \ldots$.. [n [1] we know that (in a general field theory):

0 if the system is locally conserved : the stress-energy tensor has a null divergence $\partial_{k} T^{i k}=0$;

0 and if there is "nothing (other than gravitation field)" in infinity (in the sense of convergence to infinity).

○ $\quad P^{i}(K) \equiv \frac{1}{c} \iiint \int_{\text {space-tim }, K} T^{i k} d S_{k}$ is conserved and doesn't depend on the hyperplane of integration (thanks to the conservation law).

In a less general theorem (but more old) from Von Laue (cf. [4]) we can also say that if $\partial_{0} T^{i k}=0$ (and nothing to infiny):

$$
P^{i}=\frac{1}{c} \iiint_{\text {Space }} T^{i 0} d V \text { is a 4-momentum } \Leftrightarrow \frac{1}{c} \iiint_{\text {Space }} T^{\alpha \beta} d V=0
$$




\subsection{Why (I am) searching another proof ?}

Firstly, I was often embarrassing of the proof given in some textbook where I found the demonstrations very expeditious (but of course I well understand that it is for pedagogical reasons that many authors do not expand to this particular question). Indeed we can frequently read either just the kinetic energy theorem (which is actually only one piece of reasoning conducting to the Einstein proof), either the supposition that what we can prove for a material point (for example the norm of the famous 4-momentum) can also be applied to a complex system, but this is exactly this supposition that Einstein try to prove by saying that the energy at rest (=by putting the speed equal to zero) is equal to the internal energy (see for example his 1912 Relativity unpublished manuscript [18])... However there are already general proof of the law, as the different ones given by Einstein (cf. [14]-[21] for a part of the Einstein articles and [7], [10], [12] for a review and a fine criticism) and also notably for example by the very interesting one of Langevin as presented by Yvan Simon in [5]. But despite their very convincing reasoning they are frequently applied to a particular context. Einstein use frequently an electromagnetic context (the resulting Einstein law, without any doubt, can be generally extend to any other context tanks to the direct and even indirect chain of coupling between electromagnetic field with other fields (implicit or explicit in Einstein mind)) and Langevin a thermodynamic one. These particular contexts (which again do not limit the validity of their proof) do not facilitate, for me, a clear reasoning in order to handle easily the Einstein law. Indeed, this gives me some difficulty to respond at my several personal questioning about the mass:

○ the affirmation that "the energy scale is fixed by the mass" as stated by Landau [1] but not explicitly given/proven (and not at all evident for me), the latter affirmation, was precisely used by Landau to prove the Einstein law. Indeed this affirmation, in conjunction with the calculation that $\mathrm{mc}^{2}$ (via the kinetic energy theorem) is precisely the origin of the energy scale for a material point, implies that the mass is necessary the internal energy of the eventual complex system which is contained/hidden in the material point. The problem is then to demonstrate the first Landau affirmation. This question is also related to gauge invariance(=indeterminacy of the Lagrangian), see just below.

the difference between the Einstein and the Newtonian mass: why in Newtonian theory the mass is not the origin of the energy scale ? We can say, because, the energy has no origin in mechanic. But why there is no origin in Newtonian theory but there is an origin in the Einstein one? We can respond that because the Lagrangian has some indeterminacy. But, this is also the case in Relativity....If one use the relativistic invariance to respond, we can again protest by saying that the gauge invariance of some fields (like electromagnetic field) generates also a Lagrangian indeterminacy which is already a relativistic invariant and therefore cannot be evacuated by only requiring the latter...

- to untangle a particular technical argument during the historical debate between the 2 genius Einstein \& Lemaitre about the role of the cosmological constant (the "little Lambda" discussion read in [6]) : this "technical argument" was again the origin of the energy scale and its relation with mass. Lemaître answered Einstein's criticism (the unnecessarily complexity of maintaining a not null cosmological constant, despite its natural mathematical existence) by saying that we need the cosmological constant 
partially because energy, in Relativity, is also defined with respect to an arbitrary constant. We understand that, in the Einstein gravitation theory where all energy gravitates (not only the mass, that is to say internal energy), this could worry the priest by giving rise some arbitrariness of the source of gravitation.

- The classical "renormalization" of the mass. Landau in [2] shows a divergence of any mass of a material point in Relativity due to the singular coupling between the material point and the continuous electromagnetic field. In his reasoning he warns the reader (for example after the Virial relativistic theorem proof) to pay attention to the impact of the divergence which requires the renormalization of the mass for any electromagnetic expression. Moreover in the electromagnetic radiation theory he shows an unrealistic behaviour of the material point due to the action of his own radiation on it. According to Lanfau, this behaviour is related to the difficult handling of the infinite electromagnetic mass. In order to untangle the relation with the mass, its electromagnetic divergence and other $4 / 3$ factor, it would be interesting (for pedagogical purposes) to understand a little more the useful model of electron from Lorentz \& Poincaré. This is of course already done by Poincaré in 1905 [23] and carefully analyzed in [3], [9] and [24],[25]. However, there is no discussion on the relation between the dynamics of the center of mass and the internal dynamic.

Secondly, the general proof that I gave above does not use the Lagrangian directly but indirectly via the (very useful but none-pedagogical) stress energy tensor. However, the base of all dynamics in physics laws is (until now) always to start from the Lagrangian of a system with the appropriate variables (including degrees of freedom). We should be able to select the center of mass and the complementary degrees of freedom (which we called logically the internal degrees of freedom since they are seen in the "hidden" $\mathrm{K}^{*}$ ). Unfortunately (for myself at least...), I never found any proof using this point of view. With the current approach (even if it is sufficient for physics) it is not clear, for me, how the centre of mass appears in the Lagrangian, in parallel with the internal degrees of freedom. Indeed the Lagrangian is reconstructed only a posterior, after to demonstrate that $\boldsymbol{P}_{\boldsymbol{c}}=\gamma \frac{E^{*}}{c^{2}} \boldsymbol{V}_{\mathbf{C}}$ (using the stress-energy tensor) (see [1]). So we don't clearly see the passage:

- From an initial Lagrangian $S\left[\left\{\boldsymbol{r}_{\boldsymbol{a}}(t)\right\}\right]=\int_{t_{1}}^{t_{2}} L\left(\left\{\boldsymbol{r}_{\boldsymbol{a}}\right\},\left\{\frac{d \boldsymbol{r}_{\boldsymbol{a}}}{d t}\right\}\right) d t$ or $S[\{\varphi(x, t)\}]=\frac{1}{c} \iiint \int \Lambda\left(\varphi, \frac{\partial \varphi}{\partial \boldsymbol{r}}, \frac{\partial \varphi}{\partial t}\right) d \Omega$

- To a Lagrangian of an apparent material point $S\left[\boldsymbol{R}_{\boldsymbol{c}}(t), \ldots\right]=-\int_{t_{1}}^{t_{2}} \frac{E^{*}}{\gamma\left(\boldsymbol{V}_{\boldsymbol{c}}\right)} d t+\cdots$

In this article, I propose, using directly the Lagrangian formalism with a particular choice of variable (which at first sight looks like a relativist heresy), to give the proof, for a material system (to present the method), for a field (scalar in order to simplified) and finally a system where the two interact. Moreover, in order to develop the analysis and to check the consistency of the new choice of variables introduced:

- I apply the method to the Einsteinian gravitation field;

- I show that the method permits us to observe a strong relation (an equivalence in a given context of general hypotheses) between the Einstein's $E^{*}=m c^{2}$ law and his other famous law of the time dilation. I illustrate this relation by showing how the empiric deviation of each laws should be linked;

- I carefully analyze the meaning of the particular choice of the variables in order to understand the nature of the events implied. By pushing the reasoning as much as possible in order to test 
the solidity of the reasoning, I came to a sort of a modified speed addition formula which doesn't contradict the one of Einstein-Poincare since the 2 laws are not talking about the same events;

- I also try to untangle, with this point of view, the relation between the mass and the origin of the energy scale, that is to say the meaning of the Landau affirmation that the mass is the origin of the energy scale in Relativity;

- I analyze the reason why in Newtonian mechanic we don't have an Einstein law;

- In the annex I give some elements of the Hamiltonian analysis (Hamilton and Hamilton-Jacobi equations) checking again the coherence of the particular set of variables;

- Finally, in order to apply my reasoning to a concrete example, I apply this way of thinking to the old Lorentz-Poincaré model of the electron (useful for an explicit (and so intelligible) classical renormalization of the mass). 


\section{Material system free}

\subsection{The proof for a material system}

We begin with the action principle for a set of particles:

$$
S\left[\left\{\boldsymbol{r}_{\boldsymbol{a}}(t)\right\}\right]=\int_{t_{1}}^{t_{2}} L\left(\left\{\boldsymbol{r}_{\boldsymbol{a}}\right\},\left\{\frac{d \boldsymbol{r}_{\boldsymbol{a}}}{d t}\right\}\right) d t
$$

In this expression, we are using coordinates in a Galilean reference frame K.

The degrees of freedom are the vectors $\left\{\boldsymbol{r}_{\boldsymbol{a}}\right\}$, and we integrate the expression between the plan $\mathrm{H}_{1}$ $\left(t_{1}=c t e\right)$, and $\mathrm{H}_{2}\left(t_{2}=c t e^{\prime}\right)$ in this frame.

We want now separate:

- the internal degree of freedom $\left\{\boldsymbol{r}_{\boldsymbol{a}}^{*}\right\}$, defined in the frame $\mathrm{K}^{*}$ of the center of mass ;

- from the external degree of freedom $\boldsymbol{R}_{\boldsymbol{c}}$ defined in the Galilean frame K.

So the degrees of freedom $\left\{\boldsymbol{r}_{\boldsymbol{a}}\right\}$, are equivalent to the degree o freedom $\left\{\boldsymbol{r}_{\boldsymbol{a}}^{*}, \boldsymbol{R}_{\boldsymbol{c}}\right\}$.

Note 1: a plane $t=c t e$ is seen differently for different internal particle in the frame of the center of mass $\mathrm{K}^{*}$

Thanks to the relativist invariance we know that each terms of the action associated to a particle is invariant $(L . d t=$ $\sum_{a}-m_{a} . c d s_{a}$ ). However in the frame $\mathrm{K}^{*}$, the border plan $\mathrm{H}_{1}$ and $\mathrm{H}_{2}$ are associated to different time for each particle (in Einstein Relativity the simultaneity is relative to a frame).

More explicitly, the Lorentz transformation said that a coordinate $t^{\prime}$ seen in the frame $\mathrm{K}$ is expressed like

$$
t^{\prime}-t=\gamma(t)\left(\left(t^{\prime *}-t_{c(t)}^{*}\right)+\frac{\boldsymbol{\beta}(t)}{c} \boldsymbol{r}^{*}\right)
$$

With:

- $\gamma(t)=\gamma\left(\boldsymbol{V}_{\mathbf{C}}(t)\right)$,

- $V_{\mathbf{C}} \equiv V_{\mathbf{K} * / \mathbf{K}}(t)$,

- $\quad$ and $t_{c}^{*}$, the time measured by a clock in C: $t_{c(t)}^{*}=\int_{0}^{t} \frac{d t^{\prime}}{\gamma\left(t^{\prime}\right)}$

, in the frame $\mathrm{K}^{*}(t)$ at the instant $\mathrm{t}\left(\mathrm{t}^{\prime} \neq \mathrm{t}\right.$, a priori, since $\mathrm{t}^{\prime}$ is a generic coordinate of $\mathrm{K}$ but $\mathrm{t}$ defines the time of $\mathrm{K}$ for which the center of mass has the speed $\left.\boldsymbol{V}_{\mathbf{C}}(t)\right)$.

So a plane $t^{\prime}=$ cte in $\mathrm{K}$ is seen like a plane $\gamma(t)\left(\left(t^{\prime *}-t_{c(t)}^{*}\right)+\frac{\beta}{c} \boldsymbol{r}^{*}\right)+t=c t e$ in the frame $\mathrm{K}^{*}(t)$ around $\mathrm{t}$.

Thus a particle at the position $\boldsymbol{r}_{\boldsymbol{a}}^{*}$, sees the plane $t^{\prime}=c t e$ at the instant $t^{\prime *}=\frac{t^{\prime}-t}{\gamma(t)}-\frac{\boldsymbol{V}_{\mathrm{C}}}{c^{2}} \boldsymbol{r}_{\boldsymbol{a}}^{*}+t_{c(t)}^{*}$

This is the proof of the assertion in the title.

Note 2: measurement of a clock fixed on $\mathrm{K}^{*}(t)$

Around $\mathrm{t}$ (t given and constant), a clock in $\boldsymbol{r}^{*}$ of $\mathrm{K}^{*}(t)$, and always in $\boldsymbol{r}^{*}$, measures the duration time

$t^{\prime *}-t_{c(t)}^{*}=\frac{t^{\prime}-t}{\gamma(t)}-\frac{V_{\mathrm{C}}(t)}{c^{2}} \boldsymbol{r}^{*}$ between the event $\left(c t_{c(t)}^{*}, 0^{*}\right)_{\mathrm{K} *(t)}$ associated to $\mathrm{C}$ in $\mathrm{K}^{*}(t)$ and a certain event $\left(c t^{\prime *}, \boldsymbol{r}^{*}\right)_{\mathrm{K} *(t)}$ localised, by definition, in a different position than C: that is to say $\boldsymbol{r}^{*}$.

If we demand to this clock to measures now the duration between 2 events localised in its own position, the duration is now $\Delta\left(t^{\prime *}-t_{c(t)}^{*}\right)=\Delta\left(\frac{t^{\prime}-t}{\gamma(t)}-\frac{\boldsymbol{V}_{\mathrm{C}}(t)}{c^{2}} \boldsymbol{r}^{*}\right)<=>\left(\Delta t^{\prime *}-0\right)=\left(\frac{\Delta t^{\prime}-0}{\gamma(t)}-0\right)$ since $\gamma(t), \boldsymbol{V}_{\mathbf{C}}(t), \mathrm{t}$ are constant since we work always in the same reference frame $\mathrm{K}^{*}(t)$. More over $\boldsymbol{r}^{*}=\boldsymbol{c t e}$ by definition of the 2 events considered. 
So we have $\Delta t^{\prime *}=\frac{\Delta t^{\prime}}{\gamma(t)}$ and $d t^{\prime *}=\frac{\boldsymbol{d} t^{\prime}}{\gamma(t)}$ for 2 infinitesimal events.

When we observe 2 events associated to a particle, we study the duration time between 2 hyperplanes $t^{*}=c t$ of $\mathrm{K}^{*}(t)$ where the 2 successive positions of the particle occurred. The duration is always measured by a clock fixed in $\mathrm{K}^{*}(t)$. So we can apply the relation above for the duration time associated to a particle:

$$
\forall \text { particle } a: d t_{a}^{*}=d t^{*}=\frac{d t}{\gamma(t)}
$$

\section{Note 3 : On the Lorentz transformation}

A more general Lorentz transformation is:

$$
\left(\begin{array}{c}
t_{a}-t \\
\boldsymbol{r}_{\boldsymbol{a}}\left(t_{a}\right)-\boldsymbol{R}_{\boldsymbol{c}}(t)
\end{array}\right)=L(t) \cdot\left(\begin{array}{c}
t_{a}^{*}-t_{c}^{*} \\
\boldsymbol{r}_{\boldsymbol{a}}^{*}
\end{array}\right)<=>\left\{\begin{array}{c}
t_{a}-t=\gamma(t)\left(\left(t_{a}^{*}-t_{c}^{*}\right)+\frac{\boldsymbol{\beta}}{c} \boldsymbol{r}_{\boldsymbol{a}}^{*}\right) \\
\boldsymbol{r}_{\boldsymbol{a}}\left(t_{a}\right)-\boldsymbol{R}_{\boldsymbol{c}}(t)=c\left(t_{a}^{*}-t_{c}^{*}\right) \gamma(t) \boldsymbol{\beta}+\boldsymbol{r}_{\boldsymbol{a}}^{*}+(\gamma-1) \frac{\boldsymbol{\beta}}{\beta^{2}} .\left(\boldsymbol{\beta} \boldsymbol{r}_{\boldsymbol{a}}^{*}\right)
\end{array}\right.
$$

For a movement of $\mathrm{K}^{*}$ along $\mathrm{x}$, we have the Special Lorentz transformation principally used in this article:

$$
\left\{\begin{array}{l}
t_{a}-t=\gamma(t)\left(\left(t_{a}^{*}-t_{c(t)}^{*}\right)+\frac{\beta(t)}{c} \cdot x_{a}^{*}\right) \\
x_{a}-X_{c}=\gamma(t)\left(c\left(t_{a}^{*}-t_{c(t)}^{*}\right) \beta(t)+x_{a}^{*}\right)
\end{array}\right.
$$

Now we express the action in the local frame $K^{*}(t)$ :

$$
S\left[\left\{\boldsymbol{r}_{\boldsymbol{a}}^{*}\left(t^{*}\right), \boldsymbol{R}_{\boldsymbol{c}}(t)\right\}\right]=\int_{\left\{\boldsymbol{t}_{\boldsymbol{a}, \mathbf{1}}^{*}\right\}}^{\left\{\boldsymbol{t}_{\boldsymbol{a}, 2}^{*}\right\}} L^{*}\left(\left\{\boldsymbol{r}_{\boldsymbol{a}}^{*}\right\},\left\{\frac{d \boldsymbol{r}_{\boldsymbol{a}}^{*}}{d t^{*}}\right\}, \boldsymbol{R}_{\mathbf{C}}, \boldsymbol{V}_{\mathbf{C}}\right) d t^{*}
$$

Taking account $d t^{*}=\frac{d t}{\gamma(t)}$ and returnig to the Galilean frame $\mathrm{K}$ we have:

$$
S=\int_{\left\{\boldsymbol{t}_{\boldsymbol{a}, \mathbf{1}}^{*}\right\}}^{\left\{\boldsymbol{t}_{\boldsymbol{a}, 2}^{*}\right\}} L^{*}\left(\left\{\boldsymbol{r}_{\boldsymbol{a}}^{*}\right\},\left\{\frac{d t}{d t^{*}} \frac{d \boldsymbol{r}_{\boldsymbol{a}}^{*}}{d t}\right\}, \boldsymbol{R}_{\mathbf{C}}, \boldsymbol{V}_{\mathbf{C}}\right) \frac{d t^{*}}{d t} d t=\int_{t_{1}}^{t_{2} L^{*}\left(\left\{\boldsymbol{r}_{\boldsymbol{a}}^{*}\right\},\left\{\gamma\left(\boldsymbol{V}_{\mathbf{C}}\right) \frac{d \boldsymbol{r}_{\boldsymbol{a}}^{*}}{d t}\right\}, \boldsymbol{R}_{\mathbf{C}}, \boldsymbol{V}_{\mathbf{C}}\right)} \underset{\gamma\left(\boldsymbol{V}_{\mathbf{C}}\right)}{d t}
$$

So far, nothing new.

The important point to keep in mind is that we are not considering the variation of the internal degree of freedom $\boldsymbol{r}_{\boldsymbol{a}}^{*}$ :

- relative to the internal time $t^{*}$ of $\mathrm{K}^{*}: \frac{d \boldsymbol{r}_{a}^{*}}{d t^{*}}$;

- but instead relative to time $\mathrm{t}$ of $\mathrm{K}: \frac{d \boldsymbol{r}_{a}^{*}}{d t}$.

That is to say, the Lagrangian considered is $L^{\prime}\left(\left\{\boldsymbol{r}_{\boldsymbol{a}}^{*}\right\},\left\{\frac{d \boldsymbol{r}_{\boldsymbol{a}}^{*}}{d t}\right\}, \boldsymbol{R}_{\mathbf{C}}, \boldsymbol{V}_{\mathbf{C}}\right) \equiv \frac{L^{*}\left(\left\{\boldsymbol{r}_{\boldsymbol{a}}^{*}\right\},\left\{\gamma\left(\boldsymbol{V}_{\mathbf{C}}\right) \frac{d \boldsymbol{r}_{\boldsymbol{a}}^{*}}{d t}\right\}\right)}{\gamma\left(\boldsymbol{V}_{\mathbf{C}}\right)}$, instead of

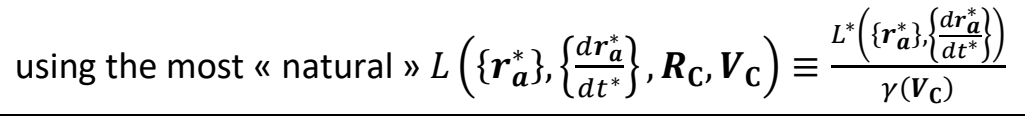

So, we can now calculate the momentum of the center of mass, with $\boldsymbol{V}_{\mathbf{C}} \equiv \boldsymbol{V}_{\mathrm{K} * / \mathbf{K}}$ : 


$$
\begin{gathered}
\boldsymbol{P}_{\boldsymbol{c}} \equiv \frac{\partial L^{\prime}}{\partial \boldsymbol{V}_{\mathrm{C}}}=\frac{\partial}{\partial \boldsymbol{V}_{\mathrm{C}}} \frac{L^{*}\left(\left\{\boldsymbol{r}_{\boldsymbol{a}}^{*}\right\},\left\{\gamma\left(\boldsymbol{V}_{\mathrm{C}}\right) \frac{d \boldsymbol{r}_{\boldsymbol{a}}^{*}}{d t}\right\}\right)}{\gamma\left(\boldsymbol{V}_{\mathrm{C}}\right)} \\
=L^{*}\left(\left\{\boldsymbol{r}_{\boldsymbol{a}}^{*}\right\},\left\{\gamma\left(\boldsymbol{v}_{\mathrm{C}}\right) \frac{d \boldsymbol{r}_{\boldsymbol{a}}^{*}}{d t}\right\}\right) \frac{\partial}{\partial \boldsymbol{v}_{\mathrm{C}}} \frac{1}{\gamma\left(\boldsymbol{V}_{\mathrm{C}}\right)}+\frac{1}{\gamma\left(\boldsymbol{V}_{\mathrm{C}}\right)} \frac{\partial}{\partial \boldsymbol{v}_{\mathrm{C}}} L^{*}\left(\left\{\boldsymbol{r}_{\boldsymbol{a}}^{*}\right\},\left\{\gamma\left(\boldsymbol{V}_{\mathrm{C}}\right) \frac{d \boldsymbol{r}_{\boldsymbol{a}}^{*}}{d t}\right\}\right)
\end{gathered}
$$

- $\frac{\partial}{\partial v_{\mathrm{c}}} \frac{1}{\gamma\left(V_{\mathrm{c}}\right)}=\frac{\partial}{\partial V_{\mathrm{c}}} \sqrt{1-\frac{V_{\mathrm{c}}^{2}}{c^{2}}}=\frac{-\frac{1}{2} 2 \frac{V_{\mathrm{C}}}{c^{2}}}{\sqrt{1-\frac{v_{\mathrm{c}}^{2}}{c^{2}}}}=-\gamma\left(V_{\mathrm{C}}\right) \frac{V_{\mathrm{c}}}{c^{2}}$

- $\frac{\partial}{\partial v_{\mathrm{c}}} L^{*}\left(\left\{\boldsymbol{r}_{a}^{*}\right\},\left\{\gamma\left(\boldsymbol{V}_{\mathrm{c}}\right) \frac{d \boldsymbol{r}_{a}^{*}}{d t}\right\}\right)=\sum_{a} \frac{\partial \gamma\left(v_{\mathrm{c}}\right) \frac{d r_{a}^{*}}{\partial t}}{\partial v_{\mathrm{c}}} \frac{\partial L^{*}}{\partial \gamma\left(v_{\mathrm{c}}\right) \frac{d r_{a}^{*}}{d t}}=\sum_{a} \frac{d r_{a}^{*}}{d t} \frac{\partial\left(1-\frac{v_{\mathrm{c}}^{2}}{c^{2}}\right)^{-1 / 2}}{\partial v_{\mathrm{c}}} \frac{\partial L^{*}}{\partial \frac{d r_{a}^{*}}{d t^{*}}}$

$$
\begin{aligned}
& =\sum_{a} \frac{d \boldsymbol{r}_{\boldsymbol{a}}^{*}}{d t}\left(\frac{\frac{1}{2} 2 \frac{\boldsymbol{V}_{\mathrm{C}}}{\boldsymbol{c}^{2}}}{\left(1-\frac{\boldsymbol{V}_{\mathrm{C}}^{2}}{\boldsymbol{c}^{2}}\right)^{3 / 2}}\right) \frac{\partial L^{*}}{\partial \frac{d \boldsymbol{r}_{\boldsymbol{a}}^{*}}{d t^{*}}}=\sum_{a} \frac{d \boldsymbol{r}_{\boldsymbol{a}}^{*}}{d t} \gamma^{3}\left(\boldsymbol{V}_{\mathrm{C}}\right) \frac{\boldsymbol{V}_{\mathrm{C}}}{\boldsymbol{c}^{2}} \frac{\partial L^{*}}{\partial \frac{d \boldsymbol{r}_{\boldsymbol{a}}^{*}}{d t^{*}}} \\
& \boldsymbol{P}_{\boldsymbol{c}}=L^{*}\left(\left\{\boldsymbol{r}_{\boldsymbol{a}}^{*}\right\},\left\{\gamma\left(\boldsymbol{V}_{\mathrm{C}}\right) \frac{d \boldsymbol{r}_{\boldsymbol{a}}^{*}}{d t}\right\}\right)\left(-\gamma\left(\boldsymbol{V}_{\mathrm{C}}\right) \frac{\boldsymbol{V}_{\mathrm{C}}}{\boldsymbol{c}^{2}}\right)+\frac{1}{\gamma\left(\boldsymbol{V}_{\mathrm{C}}\right)} \sum_{a} \frac{d \boldsymbol{r}_{\boldsymbol{a}}^{*}}{d t} \gamma^{3}\left(\boldsymbol{V}_{\mathrm{C}}\right) \frac{\boldsymbol{V}_{\mathrm{C}}}{\boldsymbol{c}^{2}} \frac{\partial L^{*}}{\partial \frac{d \boldsymbol{r}_{\boldsymbol{a}}^{*}}{d t^{*}}}
\end{aligned}
$$$$
=\gamma\left(\boldsymbol{V}_{\mathrm{C}}\right) \frac{\boldsymbol{V}_{\mathbf{C}}}{\boldsymbol{c}^{2}}\left(-L^{*}\left(\left\{\boldsymbol{r}_{\boldsymbol{a}}^{*}\right\},\left\{\gamma\left(\boldsymbol{V}_{\mathrm{C}}\right) \frac{d \boldsymbol{r}_{\boldsymbol{a}}^{*}}{d t}\right\}\right)+\sum_{a} \gamma \frac{d \boldsymbol{r}_{\boldsymbol{a}}^{*}}{d t} \frac{\partial L^{*}}{\partial \frac{d \boldsymbol{r}_{\boldsymbol{a}}^{*}}{d t^{*}}}\right)
$$$$
=\gamma\left(\boldsymbol{V}_{\mathbf{C}}\right) \frac{\boldsymbol{V}_{\mathbf{C}}}{c^{2}}\left(\sum_{a} \gamma \frac{d \boldsymbol{r}_{\boldsymbol{a}}^{*}}{d t} \frac{\partial L^{*}}{\partial \frac{d \boldsymbol{r}_{\boldsymbol{a}}^{*}}{d t^{*}}}-L^{*}\left(\left\{\boldsymbol{r}_{\boldsymbol{a}}^{*}\right\},\left\{\gamma\left(\boldsymbol{V}_{\mathbf{C}}\right) \frac{d \boldsymbol{r}_{\boldsymbol{a}}^{*}}{d t}\right\}\right)\right)
$$

$$
\begin{gathered}
=\gamma\left(\boldsymbol{V}_{\mathbf{C}}\right) \frac{V_{\mathbf{c}}}{c^{2}}\left(\sum_{a} \frac{d r_{a}^{*}}{d t^{*}} \frac{\partial L^{*}}{\partial \frac{d r_{a}^{*}}{d t^{*}}}-L^{*}\left(\left\{\boldsymbol{r}_{\boldsymbol{a}}^{*}\right\},\left\{\frac{d r_{a}^{*}}{d t^{*}}\right\}\right)\right) \text { since } \frac{d r_{a}^{*}}{d t^{*}}=\gamma\left(\boldsymbol{V}_{\mathbf{C}}\right) \frac{d r_{a}^{*}}{d t} \\
\boldsymbol{P}_{\boldsymbol{c}}=\gamma \frac{E^{*}}{c^{2}} \boldsymbol{V}_{\mathbf{C}}
\end{gathered}
$$

where $E^{*} \equiv \sum_{a} \frac{d \boldsymbol{r}_{a}^{*}}{d t^{*}} \frac{\partial L^{*}}{\partial \frac{d r_{a}^{*}}{d t^{*}}}-L^{*}\left(\left\{\boldsymbol{r}_{\boldsymbol{a}}^{*}\right\},\left\{\frac{d r_{a}^{*}}{d t^{*}}\right\}\right)$ is the internal energy.

So we have our relation.

$E^{*}$ is relative to the hyperplane $t^{*}=c t e$, the mass $M=\frac{E^{*}}{c^{2}}$ is dealing with events ( the spatiotemporal positions of the particles) simultaneous in the frame $K^{*}$ and not in the frame $K$. This is well defined since $t^{*}=\int_{0}^{t} \frac{d t^{\prime}}{\gamma\left(t^{\prime}\right)}$.

$$
M=M\left(t^{*}\right)=M\left(\int_{0}^{t} \frac{d t^{\prime}}{\gamma\left(t^{\prime}\right)}\right)
$$

We see that we don't need the 4 vector momentum to demonstrate it (we don't even use the expression $L . d t=\sum_{a}-m_{a} . c d s_{a}$ ). 
We have to note, in the proof, the importance to freeze the right variable $\left\{\frac{d r_{a}^{*}}{d t}\right\}$ (and not $\left\{\frac{d r_{a}^{*}}{d t^{*}}\right\}$ ) in order to have the good expression.

\subsection{Momentum and energy of a material system}

\subsubsection{Momentum}

We can also notice that $\boldsymbol{P}_{\boldsymbol{a}} \equiv \frac{\partial L^{\prime}}{\partial \frac{d_{a}^{*}}{d t}}=\frac{\partial \frac{L^{*}}{\gamma\left(V_{\mathbf{C}}\right)}}{\partial \frac{d r_{a}^{*}}{d t}}=\frac{1}{\gamma\left(\boldsymbol{V}_{\mathbf{C}}\right)} \frac{\partial L^{*}}{\partial \frac{r_{a}^{*}}{d t}}=\frac{\partial L^{*}}{\partial \frac{d r_{a}^{*}}{\frac{d t}{\gamma}}}=\frac{\partial L^{*}}{\partial \frac{d r_{a}^{*}}{d t^{*}}}=\boldsymbol{P}_{\boldsymbol{a}}^{*}$. We can also verify it more explicitly for notably for a free particle where we show also $\boldsymbol{P}_{\boldsymbol{a}} \equiv \frac{\partial L^{\prime}}{\partial \frac{r_{a}^{*}}{d t}}=\gamma_{a}^{*} m_{a} \cdot \frac{d \boldsymbol{r}_{a}^{*}}{d t^{*}}=\boldsymbol{P}_{\boldsymbol{a}}^{*}$.

This is surprising but reassuring for the intelligibility of this quantity: this is the same as the one we would have in the frame of the centre of mass $\mathrm{K}^{*}$.

More over the total momentum $\boldsymbol{P}_{\text {total }}$ associated to the Lagrangian $L^{\prime}\left(\left\{\boldsymbol{r}_{\boldsymbol{a}}^{*}\right\},\left\{\frac{d \boldsymbol{r}_{a}^{*}}{d t}\right\}, \boldsymbol{R}_{\mathbf{C}}, \boldsymbol{V}_{\mathbf{C}}\right)$ is $\boldsymbol{P}_{\text {total }}=\sum_{a} \frac{\partial L^{\prime}}{\partial \frac{d r_{a}^{*}}{d t}}+\frac{\partial L^{\prime}}{\partial v_{\mathrm{c}}}=\sum_{a} \boldsymbol{P}_{\boldsymbol{a}}+\boldsymbol{P}_{\boldsymbol{c}}=\boldsymbol{P}_{\boldsymbol{c}}$ since by definition of $\mathrm{K}^{*}: \sum_{a} \boldsymbol{P}_{\boldsymbol{a}} \equiv 0$. This is interesting since despite considering the internal variables on the same level as the mass center, we obtain as it should the total momentum is the one associated to the mass center.

\section{Proof:}

For a free particle we have $L \cdot d t=-\sum_{a} m_{a} \cdot c d s_{a}=>L=-\sum_{a} m_{a} \cdot c \frac{d s_{a}}{d t}=-\sum_{a} m_{a} \cdot c \frac{d s_{a}}{d t^{*}} \frac{d t^{*}}{d t}=-\sum_{a} m_{a} \cdot c^{2} \frac{1}{\gamma_{a}^{*}} \frac{1}{\gamma}$

But $\frac{1}{\gamma \cdot \gamma_{a}^{*}}=\frac{1}{\gamma} \sqrt{1-\frac{\left(\frac{d r^{*}}{d t^{*}}\right)^{2}}{c^{2}}}=\sqrt{\frac{1}{\gamma^{2}}-\frac{\frac{1}{\gamma^{2}\left(\frac{d r_{a}^{*}}{d t^{*}}\right)^{2}}}{c^{2}}}=\sqrt{\frac{1}{\gamma^{2}}-\frac{\left(\frac{d r_{a}^{*}}{d t}\right)^{2}}{c^{2}}}$ since $\frac{d r_{a}^{*}}{d t^{*}}=\gamma\left(V_{\mathrm{C}}\right) \frac{d r_{a}^{*}}{d t}$

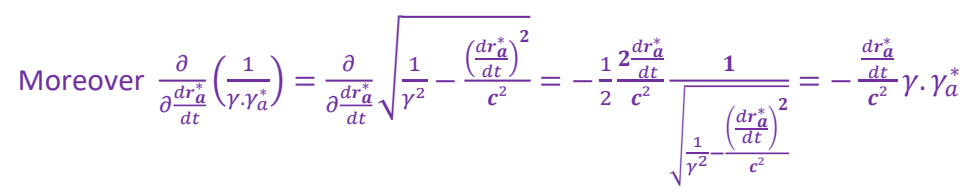

So $\boldsymbol{P}_{\boldsymbol{a}}=-\frac{\partial}{\partial \frac{d r_{a}^{*}}{d t}} \sum_{a^{\prime}} m_{a} \cdot c^{2} \frac{1}{\gamma_{a^{\prime}}^{*}} \frac{1}{\gamma}=m_{a} \cdot c^{2} \frac{\frac{d r_{a}^{*}}{d t}}{c^{2}} \gamma \cdot \gamma_{a}^{*}=m_{a} \cdot \frac{d r_{a}^{*}}{d t^{*}} \gamma_{a}^{*}$

\subsubsection{Energy}

By definition the energy associated to the Lagrangian $L^{\prime}\left(\left\{\boldsymbol{r}_{\boldsymbol{a}}^{*}\right\},\left\{\frac{d \boldsymbol{r}_{a}^{*}}{d t}\right\}, \boldsymbol{R}_{\mathbf{C}}, \boldsymbol{V}_{\mathbf{C}}\right)$ is:

$$
E^{\prime} \equiv \sum_{a} \frac{\partial L^{\prime}}{\partial \frac{d \boldsymbol{r}_{a}^{*}}{d t}} \frac{d \boldsymbol{r}_{a}^{*}}{d t}+\frac{\partial L^{\prime}}{\partial \boldsymbol{V}_{\mathrm{C}}} \boldsymbol{V}_{\boldsymbol{c}}-L^{\prime}
$$

We can re-express it as: 


$$
\begin{aligned}
& E^{\prime}=\sum_{a} \boldsymbol{P}_{\boldsymbol{a}} \frac{d \boldsymbol{r}_{\boldsymbol{a}}^{*}}{d t}+\boldsymbol{P}_{\boldsymbol{c}} \boldsymbol{V}_{\boldsymbol{c}}-\frac{L^{* \prime}}{\gamma} \text { since } L^{\prime}=\frac{L^{* \prime}}{\gamma} \\
& =\sum_{a} \frac{\partial L^{*}}{\partial \frac{d r^{*} *}{d t^{*}}} \frac{d r_{a}^{*}}{d t}+\left(\gamma \frac{E^{*}}{c^{2}} \cdot \boldsymbol{V}_{\boldsymbol{c}}\right) \boldsymbol{V}_{\boldsymbol{c}}-\frac{L^{* \prime}}{\gamma} \text { since } \boldsymbol{P}_{\boldsymbol{a}} \equiv \frac{\partial L^{\prime}}{\partial \frac{d r_{a}^{*}}{d t}}=\frac{\partial L^{*}}{\partial \frac{d r_{a}^{*}}{d t^{*}}} \\
& =\sum_{a} \frac{\partial L^{*}}{\partial \frac{d \boldsymbol{r}_{\boldsymbol{a}}^{*}}{d t^{*}}} \frac{d \boldsymbol{r}_{\boldsymbol{a}}^{*}}{d t}-\frac{L^{* \prime}}{\gamma}+\gamma \frac{E^{*}}{c^{2}} \cdot \boldsymbol{V}_{\boldsymbol{c}}^{2}
\end{aligned}
$$

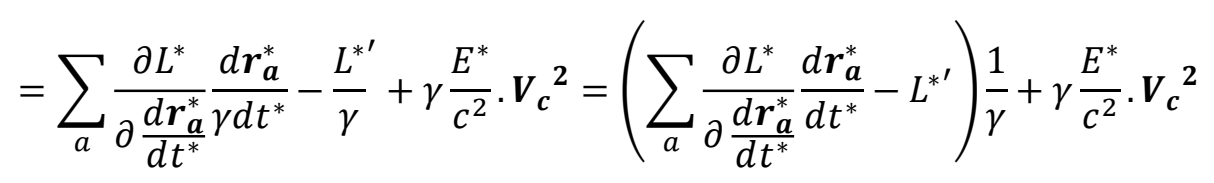

$$
\begin{aligned}
& =\frac{E^{*}}{\gamma}+\gamma \frac{E^{*}}{c^{2}} \cdot \boldsymbol{V}_{\boldsymbol{c}}{ }^{2}=\frac{E^{*}}{\gamma}+\gamma \frac{E^{*}}{c^{2}} \cdot \boldsymbol{V}_{\boldsymbol{c}}{ }^{2}=\frac{E^{*}+\gamma^{2} \frac{E^{*}}{c^{2}} \cdot \boldsymbol{V}_{\boldsymbol{c}}{ }^{2}}{\gamma}=E^{*} \frac{1+\gamma^{2} \cdot \beta^{2}}{\gamma}=E^{*} \frac{1+\frac{\beta^{2}}{1-\beta^{2}}}{\gamma} \\
& =E^{*} \frac{\frac{1-\beta^{2}+\beta^{2}}{1-\beta^{2}}}{\gamma}=E^{*} \frac{\frac{1}{1-\beta^{2}}}{\gamma}=E^{*} \frac{\gamma^{2}}{\gamma}=\gamma E^{*}
\end{aligned}
$$

We find that, as it should :

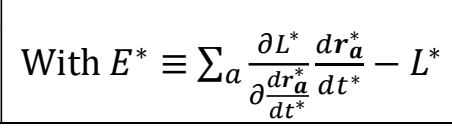

$$
E^{\prime}=\gamma E^{*}
$$

Indeed, it is the same relation that we had with the energy associated to the classical Lagrangian $L\left(\left\{\boldsymbol{r}_{\boldsymbol{a}}\right\},\left\{\frac{d \boldsymbol{r}_{a}}{d t}\right\}\right)$

$$
E \equiv \sum_{a} \frac{\partial L}{\partial \frac{d \boldsymbol{r}_{\boldsymbol{a}}}{d t}} \frac{d \boldsymbol{r}_{\boldsymbol{a}}}{d t}-L=\gamma E^{*}
$$

We can conclude that $E^{\prime}=E$

We can also conventionally note: $E=E^{*}+(\gamma-1) E^{*}$ where we observe, for a closed system $(\mathrm{E}=\mathrm{cte})$, an exchange of Energy between the internal energy $E^{*}$ and the kinetic energy $(\gamma-1) E^{*}$, the one depending of the center of mass.

\subsection{The Euler-Lagrange equation for the internal particles and the mass center}

The Euler-Lagrange equations are :

$$
\begin{aligned}
& \frac{d}{d t} \frac{\partial}{\partial \boldsymbol{V}_{\mathrm{C}}} L^{\prime}\left(\left\{\boldsymbol{r}_{\boldsymbol{a}}^{*}\right\},\left\{\frac{d \boldsymbol{r}_{\boldsymbol{a}}^{*}}{d t}\right\}, \boldsymbol{R}_{\mathrm{C}}, \boldsymbol{V}_{\mathrm{C}}\right)=\frac{\partial}{\partial \boldsymbol{R}_{\mathrm{C}}} L^{\prime}\left(\left\{\boldsymbol{r}_{\boldsymbol{a}}^{*}\right\},\left\{\frac{d \boldsymbol{r}_{\boldsymbol{a}}^{*}}{d t}\right\}, \boldsymbol{R}_{\mathrm{C}}, \boldsymbol{V}_{\mathrm{C}}\right) \\
& \forall a \frac{d}{d t} \frac{\partial}{\partial \frac{d \boldsymbol{r}_{\boldsymbol{a}}^{*}}{d t}} L^{\prime}\left(\left\{\boldsymbol{r}_{\boldsymbol{a}}^{*}\right\},\left\{\frac{d \boldsymbol{r}_{\boldsymbol{a}}^{*}}{d t}\right\}, \boldsymbol{R}_{\mathrm{C}}, \boldsymbol{V}_{\mathrm{C}}\right)=\frac{\partial}{\partial \boldsymbol{r}_{\boldsymbol{a}}^{*}} L^{\prime}\left(\left\{\boldsymbol{r}_{\boldsymbol{a}}^{*}\right\},\left\{\frac{d \boldsymbol{r}_{\boldsymbol{a}}^{*}}{d t}\right\}, \boldsymbol{R}_{\mathrm{C}}, \boldsymbol{V}_{\mathrm{C}}\right)=\frac{\partial}{\partial \boldsymbol{r}_{\boldsymbol{a}}^{*}} \frac{L^{*}\left(\left\{\boldsymbol{r}_{\boldsymbol{a}}^{*}\right\},\left\{\gamma\left(\boldsymbol{V}_{\mathrm{C}}\right) \frac{d \boldsymbol{r}_{\boldsymbol{a}}^{*}}{d t}\right\}\right)}{\gamma\left(\boldsymbol{V}_{\mathrm{C}}\right)} \\
& =\frac{1}{\gamma\left(\boldsymbol{V}_{\mathrm{C}}\right)} \frac{\partial}{\partial \boldsymbol{r}_{\boldsymbol{a}}^{*}} L^{*}\left(\left\{\boldsymbol{r}_{\boldsymbol{a}}^{*}\right\},\left\{\gamma\left(\boldsymbol{V}_{\mathrm{C}}\right) \frac{d \boldsymbol{r}_{\boldsymbol{a}}^{*}}{d t}\right\}\right)
\end{aligned}
$$

Taking account the momentum expression above we have therefore: 
$\frac{d}{d t}\left(\gamma \frac{E^{*}}{c^{2}} \boldsymbol{V}_{\mathrm{C}}\right)=\frac{\partial}{\partial \boldsymbol{R}_{\mathrm{C}}} L^{\prime}\left(\left\{\boldsymbol{r}_{\boldsymbol{a}}^{*}\right\},\left\{\frac{d \boldsymbol{r}_{\boldsymbol{a}}^{*}}{d t}\right\}, \boldsymbol{R}_{\mathrm{C}}, \boldsymbol{V}_{\mathrm{C}}\right)$

$\forall a \frac{d}{d t}\left(\gamma_{a}^{*} m_{a} \cdot \frac{d \boldsymbol{r}_{\boldsymbol{a}}^{*}}{d t^{*}}\right)=\frac{1}{\gamma\left(\boldsymbol{V}_{\mathrm{C}}\right)} \frac{\partial}{\partial \boldsymbol{r}_{\boldsymbol{a}}^{*}} L^{*}\left(\left\{\boldsymbol{r}_{\boldsymbol{a}}^{*}\right\},\left\{\gamma\left(\boldsymbol{v}_{\mathbf{C}}\right) \frac{d \boldsymbol{r}_{\boldsymbol{a}}^{*}}{d t}\right\}\right)$

As $d t^{*}=\frac{d t}{\gamma\left(v_{\mathrm{c}}\right)}$, the second equation can be re-write:

$$
<=>\frac{d}{d t^{*}}\left(\gamma_{a}^{*} m_{a} \cdot \frac{d \boldsymbol{r}_{\boldsymbol{a}}^{*}}{d t^{*}}\right)=\frac{\partial}{\partial \boldsymbol{r}_{\boldsymbol{a}}^{*}} L^{*}\left(\left\{\boldsymbol{r}_{\boldsymbol{a}}^{*}\right\},\left\{\frac{d \boldsymbol{r}_{\boldsymbol{a}}^{*}}{d t^{*}}\right\}\right)
$$

It is remarkable that we obtain the same equation that we should obtain for the dynamic in a $\mathrm{K}^{*}$ frame. However, we should notice that, since the center of mass can a priori accelerate, this is the equation for a material system in a local Galilean frame. Indeed, $d t^{*}$ is not constant as it is equal to $d t^{*}=\frac{d t}{\gamma\left(v_{\mathrm{c}}\right)}$ where $\mathrm{dt}$ is the true constant differential element. $\boldsymbol{V}_{\mathrm{C}}$ varies, so $d t^{*}$ has to vary also.

\subsection{The material system seen as a material point: the reduced action}

We can write:

$$
\begin{gathered}
S\left[\left\{\boldsymbol{r}_{\boldsymbol{a}}^{*}\left(t^{*}\right)\right\}, \boldsymbol{R}_{\boldsymbol{c}}(t)\right]=\int_{t_{1}}^{t_{2}} L^{\prime}\left(\left\{\boldsymbol{r}_{\boldsymbol{a}}^{*}\right\},\left\{\frac{d \boldsymbol{r}_{\boldsymbol{a}}^{*}}{d t}\right\}, \boldsymbol{R}_{\mathbf{C}}, \boldsymbol{V}_{\mathbf{C}}\right) d t \\
=\int_{t_{1}}^{t_{2}}\left[\sum_{a} \boldsymbol{P}_{\boldsymbol{a}} \cdot \frac{d \boldsymbol{r}_{\boldsymbol{a}}^{*}}{d t}+\boldsymbol{P}_{\boldsymbol{c}} \cdot \boldsymbol{V}_{\boldsymbol{c}}-E\right] d t \\
=\int_{t_{1}}^{t_{2}}\left[\sum_{a} \boldsymbol{P}_{\boldsymbol{a}} \cdot \frac{d \boldsymbol{r}_{\boldsymbol{a}}^{*}}{d t}+\left(\gamma \frac{E^{*}}{c^{2}} \boldsymbol{V}_{\mathbf{C}}\right) \cdot \boldsymbol{V}_{\boldsymbol{c}}-\gamma E^{*}\right] d t \\
=\int_{t_{1}}^{t_{2}}\left[\sum_{a} \boldsymbol{P}_{\boldsymbol{a}} \cdot \frac{d \boldsymbol{r}_{\boldsymbol{a}}^{*}}{d t}+\gamma E^{*}\left(\beta^{2}-1\right)\right] d t=\int_{t_{1}}^{t_{2}}\left[\sum_{a} \boldsymbol{P}_{\boldsymbol{a}} \cdot \frac{d \boldsymbol{r}_{\boldsymbol{a}}^{*}}{d t}-\frac{E^{*}}{\gamma}\right] d t
\end{gathered}
$$

So

$$
S\left[\left\{\boldsymbol{r}_{\boldsymbol{a}}^{*}\left(t^{*}\right)\right\}, \boldsymbol{R}_{\boldsymbol{c}}(t)\right]=\int_{t_{1}}^{t_{2}}\left[\sum_{a} \boldsymbol{P}_{\boldsymbol{a}} \cdot \frac{d \boldsymbol{r}_{\boldsymbol{a}}^{*}}{d t}-\frac{E^{*}}{\gamma}\right] d t
$$

If we ignore the final position of the internal degree of freedom, we have like a "spatial Maupertuis principle" (instead of a temporal used in [2]):

$$
\delta S\left[\left\{\boldsymbol{r}_{\boldsymbol{a}}^{*}\left(t^{*}\right)\right\}, \boldsymbol{R}_{\boldsymbol{c}}(t)\right]-\left(\sum_{a} \boldsymbol{P}_{\boldsymbol{a}} \cdot \delta \boldsymbol{r}_{\boldsymbol{a}}^{*}\right)_{H_{2}}=0
$$

We can see that if all the internal momentum are constant, it exists a reduced action principle:

$$
S_{0}\left[\boldsymbol{R}_{\boldsymbol{c}}(t)\right]=-\int_{t_{1}}^{t_{2}} \frac{E^{*}}{\gamma} d t
$$


We can surely generalize it for closed systems with internal separable variables where we've chosen well the variables with constant momentum. In this case, we see that for "stationary" system, in this restrict sense, the center of mass dynamic is the same as a material point.

\section{Remark on my particular choice of variables:}

My idea to consider naively the quantities $\left\{\frac{d r_{a}^{*}}{d t}\right\}$ instead of the a priori most natural $\left\{\frac{d r_{a}^{*}}{d t^{*}}\right\}$ comes to me initially from the tentative, after several unsuccessful efforts, to make appear the Lagrangian of the apparent material point with this reduced action (in the same manner we make appear the virtual work theorem: $\delta \int_{t_{1}}^{t_{2}}\left[\sum_{a} \boldsymbol{P}_{\boldsymbol{a}} \cdot d \boldsymbol{r}-H\left[\boldsymbol{P}_{\boldsymbol{a}}, \boldsymbol{r}_{\boldsymbol{a}}\right] d t\right]+\left(\sum_{a} \boldsymbol{P}_{\boldsymbol{a}} \cdot \delta \boldsymbol{r}_{\boldsymbol{a}}\right)_{H_{2}}=0$ and $\boldsymbol{P}_{\boldsymbol{a}}=\boldsymbol{c t e}=>$ $\left.\delta \int_{t_{1}}^{t_{2}} H_{\boldsymbol{P}_{\boldsymbol{a}}=c t e}\left(\left\{\boldsymbol{r}_{\boldsymbol{a}}\right\}\right) d t=0\right)$, cf .[2]).

\section{Proof:}

Indeed (do the same that [2] but for space and not for time)):

$$
\begin{gathered}
\delta S\left[\left\{\boldsymbol{r}_{\boldsymbol{a}}^{*}\left(t^{*}\right)\right\}, \boldsymbol{R}_{\boldsymbol{c}}(t)\right]-\left(\sum_{a} \boldsymbol{P}_{\boldsymbol{a}} \cdot \delta \boldsymbol{r}_{\boldsymbol{a}}^{*}\right)_{H_{2}}=0 \\
<=>\delta \int_{t_{1}}^{t_{2}} d \sum_{a}\left[\boldsymbol{P}_{\boldsymbol{a}} \cdot \boldsymbol{r}_{\boldsymbol{a}}^{*}\right]+\delta \int_{t_{1}}^{t_{2}}\left[-\frac{E^{*}}{\gamma}\right] d t-\left(\sum_{a} \boldsymbol{P}_{\boldsymbol{a}} \cdot \delta \boldsymbol{r}_{\boldsymbol{a}}^{*}\right)_{H_{2}}=0 \\
<=>\delta\left[\sum_{a} \boldsymbol{P}_{\boldsymbol{a}} \cdot \boldsymbol{r}_{\boldsymbol{a}}^{*}\right]_{H_{2}}+\delta \int_{t_{1}}^{t_{2}}\left[-\frac{E^{*}}{\gamma}\right] d t-\left(\sum_{a} \boldsymbol{P}_{\boldsymbol{a}} \cdot \delta \boldsymbol{r}_{\boldsymbol{a}}^{*}\right)_{H_{2}}=0 \\
<=>\delta \int_{t_{1}}^{t_{2}}\left[-\frac{E^{*}}{\gamma}\right] d t=0
\end{gathered}
$$


2.5. The material system seen as a material point: the internal dynamic is known

As already written:

$$
\begin{gathered}
S\left[\left\{\boldsymbol{r}_{\boldsymbol{a}}^{*}\left(t^{*}\right)\right\}, \boldsymbol{R}_{\boldsymbol{c}}(t)\right]=\int_{t_{i}}^{t_{f}} L^{\prime}\left(\left\{\boldsymbol{r}_{\boldsymbol{a}}^{*}\right\},\left\{\frac{d \boldsymbol{r}_{\boldsymbol{a}}^{*}}{d t}\right\}, \boldsymbol{R}_{\mathbf{C}}, \boldsymbol{V}_{\mathbf{C}}\right) d t \\
=\int_{t_{i}}^{t_{f}}\left[\sum_{a} \boldsymbol{P}_{\boldsymbol{a}} \cdot \frac{d \boldsymbol{r}_{\boldsymbol{a}}^{*}}{d t}-\frac{E^{*}}{\gamma}\right] d t=\int_{t_{i}}^{t_{f}}\left[\sum_{a} \gamma_{a}^{*} m_{a} \cdot \frac{d \boldsymbol{r}_{\boldsymbol{a}}^{*}}{d t^{*}} \frac{d \boldsymbol{r}_{\boldsymbol{a}}^{*}}{d t}-\frac{E^{*}}{\gamma}\right] d t
\end{gathered}
$$

We decide to say that we know the internal dynamic of the system.

That is to say we know the maps:

- $\left\{\boldsymbol{r}_{\boldsymbol{a}}^{*}\left(t^{*}\right)\right\}$

- $\left\{\frac{d r_{a}^{*}}{d t^{*}}\left(t^{*}\right)\right\}$

So, it results that the mass center is in the field (in the [2] terms) of the internal degree of freedom $\left\{\boldsymbol{r}_{\boldsymbol{a}}^{*}\right\}$. We can inject this information $\left\{\boldsymbol{r}_{\boldsymbol{a}}^{*}\left(t^{*}\right)\right\},\left\{\frac{d \boldsymbol{r}_{\boldsymbol{a}}^{*}}{d t^{*}}\left(t^{*}\right)\right\}$ in the Action :

$$
\begin{array}{r}
S\left[\left\{\boldsymbol{r}_{\boldsymbol{a}}^{*}\left(t^{*}\right)\right\}, \boldsymbol{R}_{\boldsymbol{c}}(t)\right]=\int_{t_{i}}^{t_{f}} L^{\prime}\left(\left\{\boldsymbol{r}_{\boldsymbol{a}}^{*}\right\},\left\{\frac{d \boldsymbol{r}_{\boldsymbol{a}}^{*}}{d t}\right\}, \boldsymbol{R}_{\mathbf{C}}, \boldsymbol{V}_{\mathbf{C}}\right) d t \\
=\int_{t_{i}}^{t_{f}}\left[\sum_{a} \gamma_{a}^{*} m_{a} \cdot \frac{d \boldsymbol{r}_{\boldsymbol{a}}^{*}}{d t^{*}} d \boldsymbol{r}_{\boldsymbol{a}}^{*}-\frac{E^{*}\left(t^{*}\right)}{\gamma} d t\right] \\
=\int_{\left\{\boldsymbol{t}_{\boldsymbol{a}, \boldsymbol{i}}^{*}\right\}}^{\left\{\boldsymbol{t}_{\boldsymbol{a}, \boldsymbol{f}}^{*}\right\}}\left[\sum_{a} \gamma_{a}^{*} m_{a} \cdot \frac{d \boldsymbol{r}_{\boldsymbol{a}}^{*}}{d t^{*}} d \boldsymbol{r}_{\boldsymbol{a}}^{*}\right]+\int_{t_{i}}^{t_{f}}-\frac{E^{*}\left(t^{*}\right)}{\gamma} d t \\
=\int_{\left\{\boldsymbol{t}_{\boldsymbol{a}, \boldsymbol{i}}^{*}\right\}}^{\left\{\boldsymbol{t}_{\boldsymbol{a}, \boldsymbol{f}}^{*}\right\}} d f\left\{t_{a}^{*}\right\}+\int_{t_{i}}^{t_{f}}-\frac{E^{*}\left(t^{*}\right)}{\gamma} d t
\end{array}
$$

The least action principle can therefore be express with the following action:

$$
S^{\prime \prime}\left[\boldsymbol{R}_{\boldsymbol{c}}(t), t\right]=\int_{t_{i}}^{t_{f}} L^{\prime \prime}\left(t, \boldsymbol{R}_{\mathbf{C}}, V_{\mathbf{C}}\right) d t=\int_{t_{i}}^{t_{f}}-\frac{E^{*}\left(t^{*}\right)}{\gamma\left(V_{\mathbf{C}}\right)} d t
$$

With $t^{*}=t^{*}(t)=\int_{t_{i}}^{t} \frac{d t^{\prime}}{\gamma\left(t^{\prime}\right)}$

It is important to not that we a priori don't know the expression of $t^{*}$ although we know the internal dynamic express relative to it. Indeed, knowing $t^{*}$ required to know the map $\boldsymbol{V}_{\mathbf{C}}(t)$ (part of the solution we are looking for) since $t^{*}=\int_{t_{i}}^{t} \frac{d t^{\prime}}{\gamma\left(t_{1}\right)^{\prime}}$, which is absurd. Another proof: knowing $t^{*}$, implies the undesirable consequence that $\frac{E^{*}\left(t^{*}(t)\right)}{\gamma} d t=E^{*}\left(t^{*}(t)\right) d t^{*}(t)=d f\left(t^{*}(t)\right)=d g(t)$. . This would suppress (according to the least action principle) the only one term of the action that we want to maintain in order to find the trajectory. We see therefore that the center of mass is again in the field of a variable : his own proper time $t^{*}$, as for a material point.

It seems difficult to find any relevant way in order to take account the constraint $t^{*}=\int_{t_{i}}^{t} \frac{d t^{\prime}}{\gamma\left(t^{\prime}\right)}$ in the Lagrangian. The interest to do it would be to see if the dynamic would be affected in a general way. 
Despite this problem, we can make a stronger supposition that we know, in addition to the internal dynamic, the behaviour of the energy relative to $t$ (and not): $E^{*}\left(t^{*}(t)\right)$ noted abusively $E^{*}(t)$.

Indeed even if we don't know $t^{*}(t)$ we can pretend to know $E^{*}(t)$. More precisely

$E^{*}\left(t^{*}(t)\right)=\left(E^{*} o t^{*}\right)(t)$. Knowing the map $\left(E^{*} o t^{*}\right)$ is not sufficient to know the map $t^{*}$ since the inverse map $E^{*-1}$ could eventually not exist.

Knowing $E^{*}\left(t^{*}(t)\right)$ and inject it in the Lagrangian, is equivalent to say that the center of mass is now in the field of the energy.

This situation is automatically realized in the classical case where we put $t^{*} \approx t$ in the Energy. However, we do not make the same approximation for $d t^{*}$, indeed we put $d t^{*} \approx d t\left(1-\frac{1}{2} \frac{V c^{2}}{c^{2}}\right)$. Otherwise, all the information would be lost:

we do $\frac{E^{*}\left(t^{*}(t)\right)}{\gamma} d t \approx E^{*}(t) d t\left(1+\frac{1}{2} \frac{V c^{2}}{c^{2}}\right)$ but not $\frac{E^{*}\left(t^{*}(t)\right)}{\gamma} d t \approx E^{*}(t) d t$ 
2.6. A strong link between the Einstein law and the dilation of time

$$
\begin{gathered}
\boldsymbol{P}_{\boldsymbol{c}} \equiv \frac{\partial L^{\prime}}{\partial \boldsymbol{v}_{\mathrm{C}}}=\frac{\partial}{\partial \boldsymbol{v}_{\mathrm{C}}} \frac{L^{*}\left(\left\{\boldsymbol{r}_{\boldsymbol{a}}^{*}\right\},\left\{\gamma\left(\boldsymbol{V}_{\mathrm{C}}\right) \frac{d \boldsymbol{r}_{\boldsymbol{a}}^{*}}{d t}\right\}\right)}{\gamma\left(\boldsymbol{V}_{\mathrm{C}}\right)} \\
=L^{*}\left(\left\{\boldsymbol{r}_{\boldsymbol{a}}^{*}\right\},\left\{\gamma\left(\boldsymbol{v}_{\mathrm{C}}\right) \frac{d \boldsymbol{r}_{\boldsymbol{a}}^{*}}{d t}\right\}\right) \frac{\partial}{\partial \boldsymbol{v}_{\mathrm{C}}} \frac{1}{\gamma\left(\boldsymbol{v}_{\mathrm{C}}\right)}+\frac{1}{\gamma\left(\boldsymbol{V}_{\mathrm{C}}\right)} \frac{\partial}{\partial \boldsymbol{v}_{\mathrm{C}}} L^{*}\left(\left\{\boldsymbol{r}_{\boldsymbol{a}}^{*}\right\},\left\{\gamma\left(\boldsymbol{v}_{\mathrm{C}}\right) \frac{d \boldsymbol{r}_{\boldsymbol{a}}^{*}}{d t}\right\}\right)
\end{gathered}
$$

Since in Special Relativity, the space is isotropic (इthe laws of a material system in a homogeneous \& isotropic gravitational field are isotropic) $\gamma\left(V_{C}\right)$ depends only on the norm of $\boldsymbol{V}_{\mathbf{C}}$ or equivalently on $v_{\mathrm{C}}{ }^{2}$.

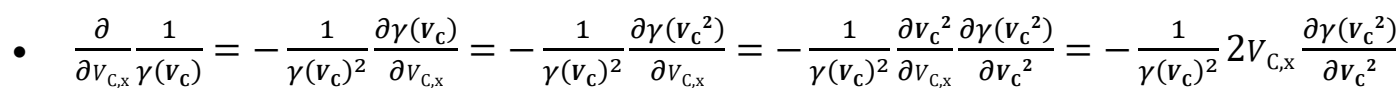

- $\frac{\partial}{\partial V_{\mathrm{C}, \mathrm{x}}} L^{*}\left(\left\{\boldsymbol{r}_{\boldsymbol{a}}^{*}\right\},\left\{\gamma\left(\boldsymbol{V}_{\mathrm{C}}\right) \frac{d r_{a}^{*}}{d t}\right\}\right)=\sum_{a} \frac{\partial \gamma\left(v_{\mathrm{c}}\right) \frac{d r_{a}^{*}}{\partial V_{\mathrm{C}, \mathrm{x}}}}{\partial \gamma\left(v_{\mathrm{c}}\right) \frac{d r_{a}^{*}}{d t}}=\sum_{a}\left(\frac{d r_{a}^{*}}{d t} \frac{\partial \gamma\left(v_{\mathrm{c}}\right)}{\partial V_{\mathrm{C}, \mathrm{x}}}\right) \frac{\partial L^{*}}{\partial \frac{d r_{a}^{*}}{d t^{*}}}=$ $\sum_{a}\left(\frac{d r_{a}^{*}}{d t} 2 V_{\mathrm{C}, \mathrm{x}} \frac{\partial \gamma\left(v_{\mathrm{c}}{ }^{2}\right)}{\partial v_{\mathrm{c}}{ }^{2}}\right) \frac{\partial L^{*}}{\partial \frac{d r_{a}^{*}}{d t^{*}}}=\sum_{a}\left(\frac{d r_{a}^{*}}{d t} 2 V_{\mathrm{C}, \mathrm{x}} \frac{\partial \gamma\left(V_{\mathrm{c}}{ }^{2}\right)}{\partial V_{\mathrm{c}}{ }^{2}}\right) \frac{\partial L^{*}}{\partial \frac{r_{a}^{*}}{d t^{*}}}$

$$
\begin{aligned}
& P_{c, x}=\frac{\partial L^{\prime}}{\partial V_{\mathrm{C}, \mathrm{x}}}=L^{*}\left(\left\{\boldsymbol{r}_{\boldsymbol{a}}^{*}\right\},\left\{\gamma\left(\boldsymbol{V}_{\mathrm{C}}\right) \frac{d \boldsymbol{r}_{\boldsymbol{a}}^{*}}{d t}\right\}\right)\left(-\frac{1}{\gamma\left(\boldsymbol{V}_{\mathrm{C}}\right)^{2}} 2 V_{\mathrm{C}, \mathrm{x}} \frac{\partial \gamma\left(\boldsymbol{V}_{\mathbf{C}}{ }^{2}\right)}{\partial \boldsymbol{v}_{\mathbf{C}}{ }^{2}}\right) \\
& +\frac{1}{\gamma\left(\boldsymbol{V}_{\mathrm{C}}\right)} \sum_{a}\left(\frac{d \boldsymbol{r}_{\boldsymbol{a}}^{*}}{d t} 2 V_{\mathrm{C}, \mathrm{x}} \frac{\partial \gamma\left(\boldsymbol{V}_{\mathrm{C}}{ }^{2}\right)}{\partial \boldsymbol{V}_{\mathrm{C}}{ }^{2}}\right) \frac{\partial L^{*}}{\partial \frac{d \boldsymbol{r}_{\boldsymbol{a}}^{*}}{d t^{*}}} \\
& =2 V_{\mathrm{C}, \mathrm{X}} \frac{\partial \gamma\left(\boldsymbol{V}_{\mathrm{C}}^{2}\right)}{\partial \boldsymbol{v}_{\mathrm{C}}{ }^{2}} \frac{1}{\gamma\left(\boldsymbol{V}_{\mathrm{C}}\right)^{2}}\left[\gamma\left(\boldsymbol{V}_{\mathrm{C}}\right) \sum_{a}\left(\frac{d \boldsymbol{r}_{\boldsymbol{a}}^{*}}{d t}\right) \frac{\partial L^{*}}{\partial \frac{d \boldsymbol{r}_{\boldsymbol{a}}^{*}}{d t^{*}}}-L^{*}\left(\left\{\boldsymbol{r}_{\boldsymbol{a}}^{*}\right\},\left\{\gamma\left(\boldsymbol{V}_{\mathrm{C}}\right) \frac{d \boldsymbol{r}_{\boldsymbol{a}}^{*}}{d t}\right\}\right)\right] \\
& =2 V_{\mathrm{C}, \mathrm{x}} \frac{\partial \gamma\left(\boldsymbol{V}_{\mathrm{C}}^{2}\right)}{\partial \boldsymbol{v}_{\mathrm{C}}{ }^{2}} \frac{1}{\gamma\left(\boldsymbol{V}_{\mathrm{C}}\right)^{2}}\left[\sum_{a} \frac{d \boldsymbol{r}_{\boldsymbol{a}}^{*}}{d t^{*}} \frac{\partial L^{*}}{\partial \frac{d \boldsymbol{r}_{\boldsymbol{a}}^{*}}{d t^{*}}}-L^{*}\left(\left\{\boldsymbol{r}_{\boldsymbol{a}}^{*}\right\},\left\{\gamma\left(\boldsymbol{v}_{\mathrm{C}}\right) \frac{d \boldsymbol{r}_{\boldsymbol{a}}^{*}}{d t}\right\}\right)\right] \\
& =2 V_{\mathrm{C}, \mathrm{x}} \frac{\partial \gamma\left(\boldsymbol{V}_{\mathrm{C}}{ }^{2}\right)}{\partial \boldsymbol{V}_{\mathrm{C}}{ }^{2}} \frac{E^{*}}{\gamma\left(\boldsymbol{V}_{\mathrm{C}}\right)^{2}}=V_{\mathrm{C}, \mathrm{x}}\left(\frac{2 c^{2}}{\gamma\left(\boldsymbol{V}_{\mathrm{C}}\right)^{2}} \frac{\partial \gamma\left(\boldsymbol{V}_{\mathrm{C}}{ }^{2}\right)}{\partial \boldsymbol{V}_{\mathrm{C}}{ }^{2}}\right) \frac{E^{*}}{c^{2}}
\end{aligned}
$$

Starting from $\boldsymbol{P}_{\boldsymbol{c}} \equiv \frac{\partial L^{\prime}\left(\left\{\boldsymbol{r}_{a}^{*}\right\},\left\{\frac{d r_{a}^{*}}{d t}\right\}, \boldsymbol{R}_{\mathrm{C}}, \boldsymbol{V}_{\mathrm{C}}\right)}{\partial V_{\mathrm{C}}}$, the fact that the space is isotropic in Special Relativity and without express explicitly $\gamma\left(\boldsymbol{V}_{\mathrm{C}}\right)$, we have:

$$
\begin{aligned}
& \boldsymbol{P}_{c}=V_{\mathrm{C}} \cdot \gamma^{e f f}\left({V_{\mathrm{c}}}^{2}\right) \frac{E^{*}}{c^{2}} \\
& \text { With } \gamma^{e f f}\left(\boldsymbol{V}_{\mathbf{C}}\right) \equiv \frac{2 c^{2}}{\gamma\left(\boldsymbol{V}_{\mathrm{c}}{ }^{2}\right)^{2}} \frac{d \gamma\left(\boldsymbol{V}_{\mathrm{c}}{ }^{2}\right)}{d \boldsymbol{V}_{\mathrm{c}}{ }^{2}}
\end{aligned}
$$

And of course $\gamma\left(V_{\mathbf{C}}{ }^{2}\right) \equiv \frac{d t}{d t^{*}}$ is dilation of time 
This is the expression of the 3-momentum of a material system without knowing explicitly the relation between the dilation of time and the speed of the mass center $\boldsymbol{V}_{\mathbf{C}}$.

Now using this general result, we want to know if the Einstein law is sufficient to obtain the right expression of the dilation of time $\gamma$ relative to $\boldsymbol{V}_{\mathbf{C}}$, that is to say the expression $\gamma\left(\boldsymbol{V}_{\mathbf{C}}{ }^{2}\right)$

We start from $\frac{E^{*}}{c^{2}}=M$. This expression means that the form of the impulsion of a system, with internal energy $E^{*}$, is the same of a material point of mass $M$ verifying $\frac{E^{*}}{c^{2}}=M$.

But for a material point we have $\boldsymbol{P}_{\boldsymbol{c}}=\boldsymbol{V}_{\mathbf{C}} \cdot \gamma\left(\boldsymbol{V}_{\mathrm{C}}{ }^{2}\right) M$, so the Einstein law implies

$$
\begin{aligned}
& \frac{E^{*}}{c^{2}}=M=>\gamma^{e f f}\left({V_{\mathrm{C}}}^{2}\right)=\gamma\left(\boldsymbol{V}_{\mathrm{C}}\right)
\end{aligned}
$$

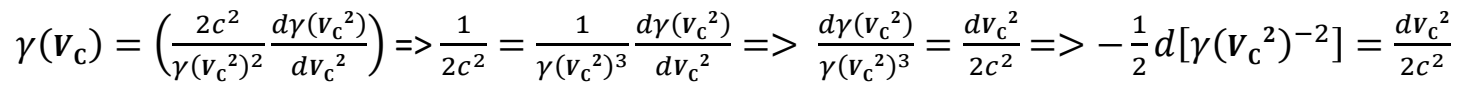

$$
\begin{aligned}
& =>-\frac{1}{2} d\left[\gamma\left(\boldsymbol{V}_{\mathrm{C}}{ }^{2}\right)^{-2}\right]=\frac{d V_{\mathrm{C}}{ }^{2}}{2 c^{2}}=>-\frac{1}{2}\left[\gamma\left(\boldsymbol{V}_{\mathrm{C}}{ }^{2}\right)^{-2}\right]_{0}^{\boldsymbol{V}_{\mathrm{C}}{ }^{2}}=\frac{\left[\boldsymbol{V}_{\mathrm{c}}{ }^{2}\right]_{0}^{\boldsymbol{V}_{\mathrm{C}}{ }^{2}}}{2 c^{2}}=>-\left[\gamma\left(\boldsymbol{V}_{\mathrm{C}}{ }^{2}\right)^{-2}-\gamma(0)^{-2}\right]=\frac{\boldsymbol{V}_{\mathrm{c}}{ }^{2}}{c^{2}} \\
& =>\gamma(0)^{-2}-\frac{\boldsymbol{V}_{\mathbf{C}}{ }^{2}}{c^{2}}=\gamma\left(\boldsymbol{V}_{\mathrm{C}}{ }^{2}\right)^{-2}=>\gamma\left(\boldsymbol{V}_{\mathrm{C}}{ }^{2}\right)=\frac{1}{\sqrt{\gamma(0)^{-2}-\frac{\boldsymbol{V}_{\mathrm{C}}{ }^{2}}{c^{2}}}} \\
& \text { But } \gamma^{e f f}(\mathbf{0})=\gamma(\mathbf{0})=1=>1=\frac{2 c^{2}}{\gamma(\mathbf{0})^{2}}\left(\frac{d \gamma\left(\boldsymbol{V}_{\mathrm{c}}{ }^{2}\right)}{d \boldsymbol{V}_{\mathbf{C}}{ }^{2}}\right)_{\boldsymbol{V}_{\mathbf{c}}{ }^{2}=0}=\frac{2 c^{2}}{\gamma(\mathbf{0})^{2}}\left(-\frac{1}{c^{2}} \frac{-1}{2} \frac{1}{\left(\gamma(0)^{-2}-\frac{V_{\mathrm{c}}{ }^{2}}{c^{2}}\right)^{3 / 2}}\right)_{\boldsymbol{V}_{\mathbf{c}}{ }^{2}=0}= \\
& \frac{c^{2}}{\gamma(\mathbf{0})^{2}} \frac{1}{c^{2}} \frac{1}{\left(\gamma(0)^{-2}\right)^{3 / 2}}=\frac{c^{2}}{\gamma(\mathbf{0})^{2}} \frac{1}{c^{2}} \gamma(0)^{3}=\gamma(0) \\
& \text { So } \frac{E^{*}}{c^{2}}=M \text { with } \boldsymbol{P}_{\boldsymbol{c}}=\boldsymbol{V}_{\mathrm{C}} \cdot \gamma\left(\boldsymbol{V}_{\mathrm{C}}{ }^{2}\right) M=>\gamma\left(\boldsymbol{V}_{\mathrm{C}}{ }^{2}\right)=\frac{1}{\sqrt{1-\frac{v^{2}}{c^{2}}}}
\end{aligned}
$$

We have the final result:

Starting from $\boldsymbol{P}_{\boldsymbol{c}} \equiv \frac{\partial L^{\prime}\left(\left\{r_{a}^{*}\right\},\left\{\frac{d r_{a}^{*}}{d t}\right\}, \boldsymbol{R}_{\mathbf{C}}, \boldsymbol{V}_{\mathbf{C}}\right)}{\partial \boldsymbol{V}_{\mathbf{c}}}$, the fact that the space is isotropic in Special Relativity and without express explicitly $\gamma\left(\boldsymbol{V}_{\mathrm{C}}\right)$, we have the equivalence:

$$
E^{*}=M c^{2}<=>\gamma\left({V_{\mathrm{C}}}^{2}\right)=\frac{1}{\sqrt{1-\frac{V_{\mathrm{C}}{ }^{2}}{c^{2}}}}
$$

With the definition

$$
\left\{\frac{E^{*}}{c^{2}}=M\right\} \equiv
$$

$\equiv\left\{\right.$ the form of the impulsion of a system, with internal energy $E^{*}$,

is the same of a material point of mass M verifying $\frac{E^{*}}{c^{2}}=M$. 
Hence the Einstein law is not only a necessary condition of special Relativity (via kinematic and least action principle), but also a sufficient condition for the dilation factor expression $\gamma\left(\boldsymbol{V}_{\mathbf{C}}{ }^{2}\right)$.

In this sense, this theorem shows that the dilation of time and the Einstein law are strongly related. So any proof of the dilation of time, is a proof of the Einstein Law and inversely.

This can also be illustrated by showing that any empirical deviation of the Einstein law $\Delta \equiv \frac{E^{*}}{c^{2}}-M$ is linked to a deviation of the Special Relativity relation $\frac{1}{\gamma\left(V_{\mathrm{C}}{ }^{2}\right)^{2}}=1-\frac{V_{\mathrm{C}}^{2}}{c^{2}}$.

$$
\begin{aligned}
& \Delta \equiv \frac{E^{*}}{c^{2}}-M=\frac{\gamma\left(\boldsymbol{V}_{\mathrm{C}}{ }^{2}\right)}{\gamma^{e f f}\left({\boldsymbol{V}_{\mathrm{C}}}^{2}\right)} M-M=M\left(\frac{\gamma\left(\boldsymbol{V}_{\mathrm{C}}{ }^{2}\right)}{\gamma^{e f f}\left(\boldsymbol{V}_{\mathrm{C}}{ }^{2}\right)}-1\right)=M\left(\frac{\gamma\left(\boldsymbol{V}_{\mathrm{C}}{ }^{2}\right)}{\frac{2 c^{2}}{\gamma\left(\boldsymbol{V}_{\mathbf{C}}{ }^{2}\right)^{2}} \frac{d \gamma\left(\boldsymbol{V}_{\mathrm{C}}{ }^{2}\right)}{d \boldsymbol{V}_{\mathrm{C}}{ }^{2}}}-1\right) \\
& =M\left(\frac{\gamma\left(\boldsymbol{V}_{\mathbf{C}}^{2}\right)^{3}}{2 c^{2} \frac{d \gamma\left(\boldsymbol{V}_{\mathrm{C}}{ }^{2}\right)}{d \boldsymbol{V}_{\mathrm{C}}{ }^{2}}}-1\right)=M\left(\frac{1}{\frac{2 c^{2}}{-2} \frac{d\left[\gamma\left(\boldsymbol{V}_{\mathrm{C}}{ }^{2}\right)^{-2}\right]}{d \boldsymbol{V}_{\mathrm{C}}{ }^{2}}}-1\right)=-M\left(1+\frac{1}{c^{2} \frac{d\left[\gamma\left(\boldsymbol{V}_{\mathbf{C}}\right)^{-2}\right]}{d \boldsymbol{V}_{\mathrm{C}}{ }^{2}}}\right)
\end{aligned}
$$

So we have

$$
\Delta \equiv \frac{E^{*}}{c^{2}}-M=-M\left(1+\frac{1}{c^{2} \frac{d\left[\gamma\left(v_{\mathrm{c}}\right)^{-2}\right]}{d v_{\mathrm{c}}{ }^{2}}}\right) \text { or } c^{2} \frac{d\left[\gamma\left(v_{\mathrm{c}}{ }^{2}\right)^{-2}\right]}{d v_{\mathrm{c}}{ }^{2}}=\left(\frac{1}{\frac{\Delta}{-M}-1}\right)=\left(\frac{-1}{1+\frac{\Delta}{M}}\right) \approx-1+\frac{\Delta}{M}
$$

If we measures $\frac{1}{\gamma\left(v_{\mathbf{c}}\right)^{2}}$ in function of ${V_{\mathbf{C}}}^{2}$ (like Bertozzi Experiment [5]), we can obtain an empiric law like

$$
\begin{aligned}
& \frac{1}{\gamma\left(v_{\mathrm{c}}{ }^{2}\right)^{2}}=\sum_{n=0}^{\infty}\left(a_{n}^{S R}+\varepsilon_{n}\right) \cdot\left\|\frac{\nu_{c}}{c}\right\|^{2 n} \text { with } a_{n}^{S R}=(1,-1,0,0,0, \ldots) \\
& \Leftrightarrow=>\frac{1}{\gamma\left(v_{\mathrm{c}}^{2}\right)^{2}}=\left(1+\varepsilon_{0}\right)+\left(-1+\varepsilon_{1}\right) \cdot\left(\frac{V_{c}}{c}\right)^{2}+\sum_{n=2}^{\infty} \varepsilon_{n} \cdot\left\|\frac{V_{c}}{c}\right\|^{2 n} \\
& =>\frac{d\left[\gamma\left(\boldsymbol{V}_{\mathbf{C}}^{2}\right)^{-2}\right]}{d \boldsymbol{V}_{\mathbf{C}}{ }^{2}}=\frac{1}{c^{2}} \frac{d\left[\gamma\left(\boldsymbol{V}_{\mathbf{C}}{ }^{2}\right)^{-2}\right]}{d\left(\frac{V_{\mathbf{C}}}{c}\right)^{2}}=\frac{1}{c^{2}}\left(\varepsilon_{1}-1+2 \sum_{n=2}^{n} \varepsilon_{n} \cdot\left\|\frac{V_{\mathbf{C}}}{c}\right\|^{2 n-1}\right)
\end{aligned}
$$

Thus we have the following relation between the empiric deviation of the 2 laws:

$$
\varepsilon_{1}+2 \sum_{n=2}^{n} \varepsilon_{n} \cdot\left\|\frac{V_{\mathrm{C}}}{c}\right\|^{2 n-1}=\frac{\Delta}{M}
$$


Any deviation of the Einstein law is linked to a deviation of the expression of the dilation of time :

$$
\varepsilon_{1}+2 \sum_{n=2}^{n} \varepsilon_{n} \cdot\left\|\frac{V_{C}}{c}\right\|^{2 n-1} \approx \frac{\Delta}{M}
$$

with

- $\Delta \equiv \frac{E^{*}}{c^{2}}-M$

- $\frac{1}{\gamma\left(v_{\mathrm{c}}\right)^{2}}=\left(1+\varepsilon_{0}\right)+\left(-1+\varepsilon_{1}\right) \cdot\left(\frac{V_{c}}{c}\right)^{2}+\sum_{n=2}^{\infty} \varepsilon_{n} \cdot\left\|\frac{V_{c}}{c}\right\|^{2 n}$

This is another way to express the link between the 2 laws. 


\subsection{Questions about the meaning of events and physical quantities used in the proof}

\subsubsection{The meaning of a speed}

There is a priori a problem with the speed $\frac{d r_{a}^{*}}{d t}$ since it combines 2 quantities that each relies to 2 different references frames: $\mathrm{K}^{*}$ for $d \boldsymbol{r}_{\boldsymbol{a}}^{*}$ and $\mathrm{K}$ for $d t$. It may be thought to be ill-defined, which would break the demonstration.

In many textbook like in [5] \& [5'] we can "traditionally" correctly write $\frac{d r_{a}^{*}}{d t}=\frac{d r_{a}^{*}}{d t^{*}} \frac{d t^{*}}{d t}$, and according to the Lorentz Transformation $\frac{d t^{*}}{d t}=\frac{1}{\gamma\left(d t^{*}+\frac{\beta}{c} d x_{a}^{*}\right)}=\frac{1}{d t_{1}+d t_{2}}$ with $d t_{1} \equiv \gamma \cdot d t^{*}$ and $d t_{2} \equiv \gamma \frac{\beta}{c} d x_{a}^{*}$.

$$
\frac{d \boldsymbol{r}_{\boldsymbol{a}}^{*}}{d t}=\frac{d \boldsymbol{r}_{\boldsymbol{a}}^{*}}{d t_{1}+d t_{2}}=\frac{d \boldsymbol{r}_{\boldsymbol{a}}^{*}}{\gamma\left(d t^{*}+\frac{\beta}{c} d x_{a}^{*}\right)}
$$

However we don't use this textbook (or traditional) formula above in this article but another instead (consequently $d \boldsymbol{r}_{\boldsymbol{a}}^{*}$ has also another meaning):

$$
\frac{d \boldsymbol{r}_{\boldsymbol{a}}^{*}}{d t}=\frac{d \boldsymbol{r}_{\boldsymbol{a}}^{*}}{d t_{1}}=\frac{d \boldsymbol{r}_{\boldsymbol{a}}^{*}}{\gamma d t^{*}}
$$

So what the 2 expressions really mean, why are we using the second whereas the first ? and is there any sense to use the second ? The latter question is important since my proof is totally based on it.

a. $\quad$ In the first expression $\frac{d r_{a}^{*}}{d t}=\frac{d r_{a}^{*}}{\gamma\left(d t^{*}+\frac{\beta}{c} d x_{a}^{*}\right)}$, we are actually using the Lorentz transformation about the 2 same events seen in 2 different Galilean Frames $\mathrm{K}$ and $\mathrm{K}^{*}$ :

- $a_{1}=\left(c t_{K_{1}^{*}}, \boldsymbol{r}_{\boldsymbol{a}, K_{1}^{*}}\left(t_{K_{1}^{*}}\right)\right)_{K_{1}^{*}}=\left(c t_{1}, \boldsymbol{r}_{\boldsymbol{a}}\left(t_{1}\right)\right)_{K}$

- $a_{2}=\left(c\left(t_{K_{1}^{*}}+d t_{K_{1}^{*}}\right), \boldsymbol{r}_{\boldsymbol{a}, K_{1}^{*}}\left(t_{K_{1}^{*}}+d t_{K_{1}^{*}}\right)\right)_{K_{1}^{*}}=\left(c\left(t_{1}+\left(t_{2}-t_{1}\right)\right), \boldsymbol{r}_{\boldsymbol{a}}\left(t_{1}\right)+d \boldsymbol{r}_{\boldsymbol{a}}\left(t_{1}\right)\right)_{K}$ Indeed, at the time $t_{1}$ of $K$ we associate to the center of mass $C$, at the position $x_{c}\left(t_{1}\right)$. Then any coordinate $\left(c t_{K_{1}^{*}}, x_{K_{1}^{*}}\right)_{K_{1}^{*}}$ of the local (current Galilean ) reference frame $\mathrm{K}^{*}$ is related to that of $\mathrm{K}$ $(c t, x)_{K}$ with the Lorentz transformation:

$$
\begin{gathered}
\left\{\begin{array}{l}
\text { c.t }- \text { c. } t_{1}=\gamma_{t_{1}} \cdot\left(c\left(t_{K_{1}^{*}}-\int_{0}^{t_{1}} \frac{d t}{\gamma_{t}}\right)+\beta_{t_{1}} \cdot\left(x_{K_{1}^{*}}-x_{C, K_{1}^{*}}\right)\right) \\
x-x_{c}\left(t_{1}\right)=\gamma_{t_{1}} \cdot\left(\left(x_{K_{1}^{*}}-x_{C, K_{1}^{*}}\right)+\beta_{t_{1}} \cdot\left(t_{K_{1}^{*}}-\int_{0}^{t_{1}} \frac{d t}{\gamma_{t}}\right)\right)
\end{array}\right. \\
\quad \Leftrightarrow=\left\{\begin{array}{l}
c . t-c . t_{1}=\gamma_{t_{1}} \cdot\left(c\left(t_{K_{1}^{*}}-t_{C\left(t_{1}\right)}^{*}\right)+\beta_{t_{1}} \cdot x_{K_{1}^{*}}\right) \\
x-x_{c}\left(t_{1}\right)=\gamma_{t_{1}} \cdot\left(x_{K_{1}^{*}}+\beta_{t_{1}} \cdot\left(t_{K_{1}^{*}}-t_{C\left(t_{1}\right)}^{*}\right)\right)
\end{array}\right.
\end{gathered}
$$

- $t_{C\left(t_{1}\right)}^{*} \equiv \int_{0}^{t_{1}} \frac{d t}{\gamma_{t}}$ the time seen from the clock in C ;

- $x_{C, K_{1}^{*}} \equiv 0$ by deciding that $\mathrm{C}$ is the spatial origin of the current $\mathrm{K}^{*}$.

- $\gamma_{t_{1}}, t_{C\left(t_{1}\right)}^{*}, \beta_{t_{1}}$ are constants associated to the Lorentz transformation at the time $t_{1}$.

So we simply apply this transformation for the 2 events:

- On one hand:

$\mathrm{d}\left(c . t-c . t_{1}\right) \equiv\left(c . t-c . t_{1}\right)_{a_{2}}-\left(c . t-c . t_{1}\right)_{a_{1}}=(c . t)_{a_{2}}-c . t_{1}-(c . t)_{a_{1}}+c . t_{1}=c . d t$

- On the other hand: $\left(c . t-c . t_{1}\right) a_{2}-\left(c . t-c . t_{1}\right) a_{1}=$

$$
=\left[\gamma_{t_{1}} \cdot\left(c\left(t_{K_{1}^{*}}-t_{C\left(t_{1}\right)}^{*}\right)+\beta_{t_{1}} \cdot x_{K_{1}^{*}}\right)\right]_{a_{2}}-\left[\gamma_{t_{1}} \cdot\left(c\left(t_{K_{1}^{*}}-t_{C\left(t_{1}\right)}^{*}\right)+\beta_{t_{1}} \cdot x_{K_{1}^{*}}\right)\right]_{a_{1}}
$$

$=\gamma_{t_{1}}\left[\left(c\left(t_{K_{1}^{*}, a_{2}}-t_{K_{1}^{*}, a_{1}}\right)+\beta_{t_{1}} \cdot\left(x_{K_{1}^{*}, a_{2}}-x_{K_{1}, a_{1}}\right)\right)\right]$ since $\gamma_{t_{1}}, t_{C}^{*} \& \beta_{t_{1}}$ are constant

$=\gamma_{t_{1}}\left(c d t_{K_{1}^{*}}+\beta_{t_{1}} \cdot d x_{K_{1}^{*}}\right)$ 
So we got what we expected $c . d t=\gamma_{t_{1}}\left(c . d t_{K_{1}^{*}}+\beta_{t_{1}} \cdot d x_{K_{1}^{*}}\right)$

b. Now what is the meaning of the second expression $\frac{d r_{a}^{*}}{d t}=\frac{d r_{a}^{*}}{d t_{1}}=\frac{d r_{a}^{*}}{\gamma d t^{*}}$ ?

The answer of the question need to clarify what we are actually doing in the reasoning of this article. First, we start to suppose the knowledge of the movement of the center of mass $C$, for each time $t$ of $\mathrm{K}$. This knowledge imposes the movement of the reference frame $\mathrm{K}^{*}$ since we choose to define it such that, around each time $t$, it coincides with the family of Galilean reference frame $\left(\mathrm{K}^{*}(\mathrm{t})\right)_{\mathrm{t} \in \mathrm{R}}$

0 in a uniform rectilinear translation relative to $\mathrm{K}$ ( with the speed of $\mathrm{C}: \boldsymbol{V}_{\boldsymbol{C} / \mathbf{K}^{*}}$ );

$\circ$ and having for spatial origin the position of $\mathrm{C}$.

So we have parameterized the reference frame $\mathrm{K}^{*}$ with the time $t_{i}$ of $\mathrm{K}$ with a map, say $\mathrm{g}$ :

$$
\boldsymbol{g}: t_{i} \rightarrow K^{*}\left(t_{i}\right) \text { also noted } K_{i}^{*}
$$

Secondly, what are the events involved in the two frames? We are studying a particle "a" of a material system with $C$ as its mass center. We can a priori think that, at the instant $t_{1}$ of $K$, since we study an event $\left(c t_{1}, x_{a}\left(t_{1}\right)\right)_{K}$, we have to study in $K^{*}\left(t_{1}\right)$ the same event seen with the different coordinate due to the direct application of the Lorentz transformation to $\left(c t_{1}, x_{a}\left(t_{1}\right)\right)_{K} \ldots$ But it is actually not the case.

Indeed, at the instant $t_{1}$ of $\mathrm{K}$ we apply the map $\mathbf{g}$ defined above and we observe in $K^{*}\left(t_{1}\right)$ all the elements which are simultaneous with the event associated to the spatio-temporal position of $C$ : $\left(c t_{1}, x_{c}\right)_{K}$.

So contrary to the case 1 ), in the case 2 ): we are not studying the same event (the same spatiotemporal position of the particle "a") in two different frame but :

- An event $\left(c t_{1}, x_{a}\left(t_{1}\right)\right)_{K}$ in $\mathrm{K}$;

- And an event $\mathrm{E}_{1}=\left(c \cdot t_{C\left(t_{1}\right)}^{*}, x_{a, K_{1}^{*}}\right)_{K_{1}^{*}}$ in $K^{*}\left(t_{1}\right)$ defined by its simultaneity with $\left(c t_{1}, x_{c}\right)_{K}$.

By the relativity of the simultaneity, this event $\mathrm{E}_{1}$ in $K^{*}\left(t_{1}\right)$ cannot be associated to the instant $t_{1}$ of $\mathrm{K}$. In fact, only the event $\left(c t_{1}, x_{c}\right)_{K}$ is analysed with the two reference frame $\mathrm{K} \& K^{*}\left(t_{1}\right)$. So we understand why we cannot use the expression of the case a).

In order to visualize the situation, we show below the schematic view of what we are truly doing. 


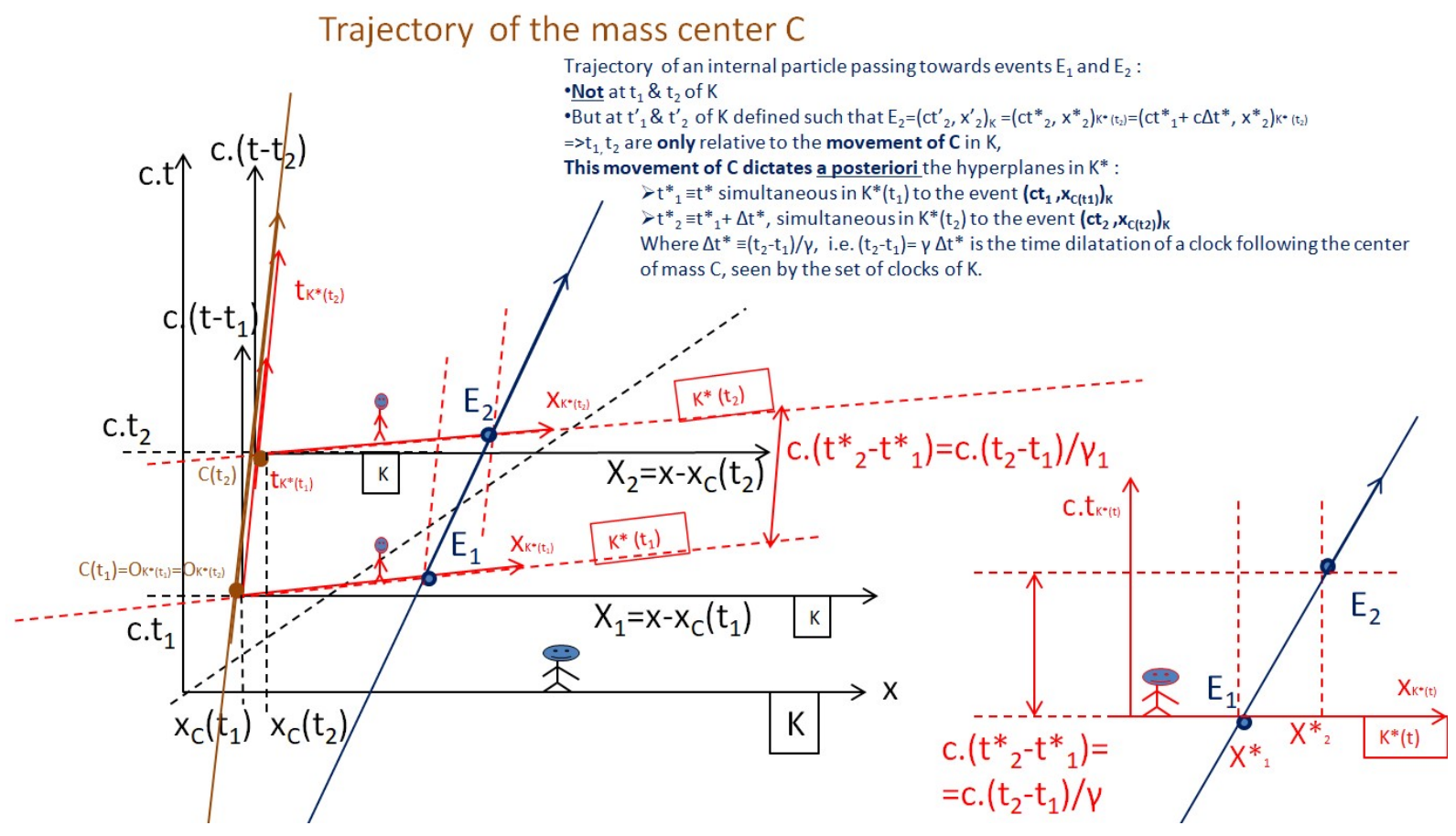

This schematic view use the 2 following expressions calculated in ANNEX:

- $\quad c \cdot t_{\left(x_{K_{i}^{*}}^{*}=c t e\right)}(x)=c \cdot t_{i}+\frac{x-x_{c}\left(t_{i}\right)}{\beta_{t_{i}}}-\frac{c t e}{\gamma_{t_{i}} \cdot \beta_{t_{i}}}$

- $\quad c . t_{\left(c t_{K_{i}^{*}}^{*}=c t e\right)}(x)=c \cdot t_{i}+\beta_{t_{i} \cdot}\left(x-x_{c}\left(t_{i}\right)\right)+\frac{c\left(c t e-t_{C\left(t_{i}\right)}^{*}\right)}{\gamma_{t_{i}}}$

We also use the fact that, according to the definition of the reference frame of the centre of mass, the orientation all the hyperplane of simultaneity of $K^{*}\left(t_{1}\right)$ are (around $t_{1}$ ):

○ the hyperplanes $t_{K_{1}^{*}}=t_{C\left(t_{1}\right)}^{*}$

$\bigcirc \quad$ and all the other separated by $d t_{K_{1}^{*}}=\frac{d t}{\gamma_{t_{1}}}$

Indeed, thanks to the Lorentz transformation between the reference frame $\mathrm{K}$ and $K_{i}^{*} \equiv K^{*}\left(t_{i}\right)$

$$
\begin{gathered}
\left\{\begin{array}{l}
c . t-c . t_{i}=\gamma_{t_{i}} \cdot\left(\mathrm{c}\left(t_{K_{i}^{*}}-t_{C\left(t_{i}\right)}^{*}\right)+\beta_{t_{i}} \cdot x_{K_{i}^{*}}\right) \\
x-x_{c}\left(t_{i}\right)=\gamma_{t_{i}} \cdot\left(x_{K_{i}^{*}}+\beta_{t_{i}} \cdot\left(t_{K_{i}^{*}}-t_{C\left(t_{i}\right)}^{*}\right)\right)
\end{array},\right. \text { we have } \\
\text { c.t }- \text { c. } t_{i}=\gamma_{t_{i}} \cdot\left(\mathrm{c}\left(t_{K_{i}^{*}}-t_{C\left(t_{i}\right)}^{*}\right)+\beta_{t_{i}} \cdot x_{K_{i}^{*}}\right)=>c . t=c . t_{i}+\gamma_{t_{i}} \cdot\left(\mathrm{c}\left(t_{K_{i}^{*}}-t_{C\left(t_{i}\right)}^{*}\right)+\beta_{t_{i}} \cdot x_{K_{i}^{*}}\right) \\
=>c . t_{\left(x_{K_{i}^{*}}=c t e\right)}\left(\mathrm{c} t_{K_{i}^{*}}\right)=c . t_{i}+\gamma_{t_{i}} \cdot\left(\mathrm{c}\left(t_{K_{i}^{*}}-t_{C\left(t_{i}\right)}^{*}\right)+\beta_{t_{i}} \cdot c t e\right)
\end{gathered}
$$

Its results that relative to $\mathrm{K}$, events situated, at rest, at the origin of $K_{1}^{*}$ (that is to say $\mathrm{C}$ ) and having the time $t_{K_{i}^{*}}$ are observed at the time $t_{\left(x_{K_{1}^{*}}=0\right)}\left(c t_{K_{i}^{*}}\right)=t_{1}+\gamma_{t_{1}} \cdot\left(t_{K_{1}^{*}}-t_{C\left(t_{1}\right)}^{*}\right)$.

This situation is of course relevant for the centre of mass $C$ between the instant $t_{1}$ and $t_{2}$ :

$$
t_{2}-t_{1}=\gamma_{t_{1}} \cdot\left(t_{K_{2}^{*}}-t_{1, K_{1}^{*}}\right)<=>t_{K_{1}^{*}}-t_{C\left(t_{1}\right)}^{*}=\frac{t_{2}-t_{1}}{\gamma_{t_{1}}}
$$


This relation also relevant to all couples of events having the same position (at rest) in $K^{*}\left(t_{1}\right)$. So, we have the relation affirmed in 2) and showed in the picture above.

The particle event of the reference frame $\mathrm{K}^{*}$ are also parameterized by the time $t$ of $\mathrm{K}$ Indeed, we can define for a particle "a" a map:

$$
\boldsymbol{g}_{\boldsymbol{a}}: t_{i} \rightarrow \mathrm{E}_{t_{i}}=\left(c . t_{C\left(t_{i}\right)}^{*}, x_{a, K_{i}^{*}}\right)_{K_{1}^{*}}
$$

That is to say, at each time $t_{i}$ of $\mathrm{K}$, we associate a frame $K^{*}\left(t_{i}\right)$, then the event $\mathrm{E}_{t_{i}}$ associated to the particle is the one localized in the hyperplane of $K^{*}\left(t_{i}\right)$ which contain also $\mathbf{C}$ at the instant $t_{i}$.

We are not saying that the particle "a" is seen at the instant $t_{i}$ in $K^{*}\left(t_{i}\right)$ (a non-sense in Relativity) but instead it is associated to the instant $t_{i}$ in the map $\boldsymbol{g}_{\boldsymbol{a}}$ sense: indeed, the hyperplane of simultaneity of $K^{*}\left(t_{i}\right)$ is parameterized by $t_{i}$.

In order to more untangle these relations, we give just below the explicit expression of $\mathrm{E}_{i}=\mathrm{E}_{t_{i}}$ in $\mathrm{K}$. To insist in the fact that $\mathrm{E}_{i}$ is parameterized by the time $t_{i}$, I will always write it $\mathrm{E}_{t_{i}}$.

\subsubsection{What is the coordinates of $E_{t 1}$ in $K$ ?}

We suppose the knowledge of the trajectory of $\mathrm{C}$ and the internal particle "a" relative to $\mathrm{K} x_{a}(t)$.

At $t_{1}, \mathrm{E}_{t_{1}}$ has the same plane $c . t^{*}=c t_{K_{1}^{*}}$ than $\mathrm{C}$ which has the coordinate $\left(c t_{C\left(t_{1}\right)}^{*}, 0^{*}\right)_{K_{1}^{*}}=$ $\left(c . \int_{0}^{t_{1}} \frac{d t}{\gamma_{t}}, 0^{*}\right)_{K_{1}^{*}}$ in $K^{*}\left(t_{1}\right)$.

Moreover at a given coordinate $\mathrm{x}$ of " $\mathrm{a}$ " in $\mathrm{K}$ we have:

$$
\text { c. } t_{\left(c . t^{*}=c t_{C\left(t_{1}\right)}^{*}\right)}(x)=c . t_{1}+\beta_{t_{1}} \cdot\left(x-x_{c}\left(t_{1}\right)\right)
$$

\section{What can we choose for $x$ ?}

The expression was calculated for a particle " $a$ " on the $\mathrm{x}$-axis of $\mathrm{K}$ at a time of $\mathrm{K}$ where the function $x_{a}(t)$ is the $x$-coordinate associate to $c . t_{\left(c . t^{*}=c t_{C\left(t_{1}\right)}^{*}\right.}$ which different from $t_{1}$ with a certain duration $\Delta t_{1}$. The time of $\mathrm{K}$ where $\mathrm{E}_{t_{1}}$ tooks place is :

$$
\text { c. } t_{\left(c . t^{*}=c t_{C\left(t_{1}\right)}^{*}\right)}\left(x_{a}\left(t_{1}+\Delta t_{1}\right)\right)=c . t_{1}+\beta_{t_{1}} \cdot\left(x_{a}\left(t_{1}+\Delta t_{1}\right)-x_{c}\left(t_{1}\right)\right)
$$

We can notice that, knowing the trajectories $x_{a}(t), x_{c}(t), \Delta t_{1}$ is a solution of the equation:

$$
\Delta t_{1}=\frac{\beta_{t_{1}}}{c} \cdot\left(x_{a}\left(t_{1}+\Delta t_{1}\right)-x_{c}\left(t_{1}\right)\right)
$$

- In a particular case where $x_{a}\left(t_{1}+\Delta t_{1}\right)$ can be developed at the first order, the latter equation is reduced to:

$$
\begin{gathered}
\Delta t_{1}{ }^{(1)} \approx \frac{\beta_{t_{1}}}{c} \cdot\left(x_{a}\left(t_{1}\right)+\frac{d x_{a}}{d t}\left(t_{1}\right) \Delta t_{1}{ }^{(1)}-x_{c}\left(t_{1}\right)\right) \\
<=>\Delta t_{1}{ }^{(1)}\left(1-\frac{\beta_{t_{1}}}{c} \frac{d x_{a}}{d t}\left(t_{1}\right)\right) \approx \frac{\beta_{t_{1}}}{c} \cdot\left(x_{a}\left(t_{1}\right)-x_{c}\left(t_{1}\right)\right) \\
<=>\Delta t_{1} \approx \Delta t_{1}{ }^{(1)} \equiv \frac{\beta_{t_{1}}}{c} \cdot \frac{x_{a}\left(t_{1}\right)-x_{c}\left(t_{1}\right)}{1-\beta_{t_{1}} \frac{V_{a}}{c}\left(t_{1}\right)}
\end{gathered}
$$


- In a particular case where $x_{a}\left(t_{1}+\Delta t_{1}\right)$ can be developed at the second order, the latter equation is reduced to:

$$
\begin{aligned}
& \Delta t_{1}{ }^{(2)} \approx \frac{\beta_{t_{1}}}{c} \cdot\left(x_{a}\left(t_{1}\right)+\frac{d x_{a}}{d t}\left(t_{1}\right) \cdot \Delta t_{1}{ }^{(2)}+\frac{d^{2} x_{a}}{d t^{2}}\left(t_{1}\right) \cdot \frac{\Delta t_{1}{ }^{(2)^{2}}}{2}-x_{c}\left(t_{1}\right)\right) \\
<= & 0 \approx\left[\frac{\beta_{t_{1}}}{2 c} \frac{d^{2} x_{a}}{d t^{2}}\left(t_{1}\right)\right] \Delta t_{1}{ }^{(2)^{2}}+\left[\frac{\beta_{t_{1}}}{c} \frac{d x_{a}}{d t}\left(t_{1}\right)-1\right] \Delta t_{1}{ }^{(2)}+\frac{\beta_{t_{1}}}{c}\left[x_{a}\left(t_{1}\right)-x_{c}\left(t_{1}\right)\right] \\
<=> & 0 \approx\left[\frac{\beta_{t_{1}}}{2} \frac{a_{a}}{c}\left(t_{1}\right)\right] \Delta t_{1}{ }^{(2)^{2}}-\left[1-\beta_{t_{1}} \frac{V_{a}}{c}\left(t_{1}\right)\right] \Delta t_{1}{ }^{(2)}+\Delta t_{1}{ }^{(1)}\left(1-\beta_{t_{1}} \frac{V_{a}}{c}\left(t_{1}\right)\right)
\end{aligned}
$$

We can try to solve it directly, using the standard solution of the second order equation, but it should be not useful since the solution will not be applicable in the usual case where there is no acceleration... However, there is another way to solve it with the perturbation $\varepsilon$ of the first order solution $\Delta t_{1}{ }^{(1)}: \Delta t_{1}{ }^{(2)}=\Delta t_{1}{ }^{(1)}+\varepsilon$

$$
\begin{gathered}
0 \approx\left[\frac{\beta_{t_{1}}}{2} \frac{a_{a}}{c}\left(t_{1}\right)\right] \Delta t_{1}{ }^{(2)^{2}}-\left(\Delta t_{1}{ }^{(2)}-\Delta t_{1}{ }^{(1)}\right)\left(1-\beta_{t_{1}} \frac{V_{a}}{c}\left(t_{1}\right)\right) \\
\Leftrightarrow=\Delta t_{1}{ }^{(2)}-\Delta t_{1}{ }^{(1)} \approx \frac{\left[\frac{\beta_{t_{1} a_{a}}}{c}\left(t_{1}\right)\right]}{1-\beta_{t_{1}} \frac{V a}{c}\left(t_{1}\right)} \Delta t_{1}{ }^{(2)^{2}}
\end{gathered}
$$

Using $\Delta t_{1}{ }^{(2)}=\Delta t_{1}{ }^{(1)}+\varepsilon$, we have:

$$
\begin{aligned}
& \varepsilon \approx \frac{\left[\frac{\beta_{t_{1}}}{2} \frac{a_{a}}{c}\left(t_{1}\right)\right]}{1-\beta_{t_{1}} \frac{V_{a}}{c}\left(t_{1}\right)}\left(\Delta t_{1}{ }^{(1)}+\varepsilon\right)^{2}=\frac{\left[\frac{\beta_{t_{1}}}{2} \frac{a_{a}}{c}\left(t_{1}\right)\right]}{1-\beta_{t_{1}} \frac{V_{a}}{c}\left(t_{1}\right)}\left(\Delta t_{1}{ }^{(1)^{2}}+\varepsilon^{2}+2 \varepsilon \Delta t_{1}{ }^{(1)}\right) \\
& <=>\varepsilon=\frac{\left[\frac{\beta_{t_{1}}}{2} \frac{a_{a}}{c}\left(t_{1}\right)\right]}{1-\beta_{t_{1}} \frac{V_{a}}{c}\left(t_{1}\right)}\left(\Delta t_{1}{ }^{(1)^{2}}+\varepsilon^{2}+2 \varepsilon \Delta t_{1}{ }^{(1)}\right) \\
& <=>\varepsilon \approx \frac{\left[\frac{\beta_{t_{1} a_{a}}}{2 c}\left(t_{1}\right)\right]}{1-\beta_{t_{1}} \frac{V a}{c}\left(t_{1}\right)}\left(\Delta t_{1}{ }^{(1)^{2}}+2 \varepsilon \Delta t_{1}{ }^{(1)}\right) \text { with } \Delta t_{1}{ }^{(1)} \gg \varepsilon \\
& <=>\varepsilon\left(1-2 \Delta t_{1}{ }^{(1)} \frac{\left[\frac{\beta_{t_{1}}}{2} \frac{a_{a}}{c}\left(t_{1}\right)\right]}{1-\beta_{t_{1}} \frac{V_{a}}{c}\left(t_{1}\right)}\right) \approx \frac{\left[\frac{\beta_{t_{1}}}{2} \frac{a_{a}}{c}\left(t_{1}\right)\right]}{1-\beta_{t_{1}} \frac{V_{a}}{c}\left(t_{1}\right)} \Delta t_{1}^{(1)^{2}} \\
& <=>\varepsilon \approx \frac{\frac{\left[\frac{\beta_{t_{1}}}{2} \frac{a_{a}}{c}\left(t_{1}\right)\right]}{1-\beta_{t_{1}} \frac{V_{a}}{c}\left(t_{1}\right)}}{1-2 \Delta t_{1}{ }^{(1)} \frac{\left[\frac{\beta_{t_{1}}}{2} \frac{a_{a}}{c}\left(t_{1}\right)\right]}{1-\beta_{t_{1}} \frac{V_{a}}{c}\left(t_{1}\right)}} \Delta t_{1}{ }^{(1)^{2}}
\end{aligned}
$$




$$
=>\varepsilon \approx \frac{\left[\frac{\beta_{t_{1}}}{2} \frac{a_{a}}{c}\left(t_{1}\right)\right]}{1-\beta_{t_{1}} \frac{V_{a}}{c}\left(t_{1}\right)} \cdot \Delta t_{1}^{(1)^{2}}\left(1+2 \Delta t_{1}{ }^{(1)} \frac{\left[\frac{\beta_{t_{1}}}{2} \frac{a_{a}}{c}\left(t_{1}\right)\right]}{1-\beta_{t_{1}} \frac{V_{a}}{c}\left(t_{1}\right)}\right) \approx \frac{\left[\frac{\beta_{t_{1}}}{2} \frac{a_{a}}{c}\left(t_{1}\right)\right]}{1-\beta_{t_{1}} \frac{V_{a}}{c}\left(t_{1}\right)} \cdot \Delta t_{1}{ }^{(1)^{2}}
$$

$$
\begin{gathered}
\Delta t_{1}^{(2)}=\Delta t_{1}^{(1)}+\frac{\left[\frac{\beta_{t_{1}}}{2} \frac{a_{a}}{c}\left(t_{1}\right)\right]}{1-\beta_{t_{1}} \frac{V_{a}}{c}\left(t_{1}\right)} \Delta t_{1}{ }^{(1)^{2}} \\
\text { With } \Delta t_{1}^{(1)} \equiv \frac{\beta_{t_{1}}}{c} \cdot \frac{x_{a}\left(t_{1}\right)-x_{c}\left(t_{1}\right)}{1-\beta_{t_{1}} \frac{V a}{c}\left(t_{1}\right)}
\end{gathered}
$$

The traditional calculation gives:

$$
\begin{aligned}
& \Delta=\left[1-\beta_{t_{1}} \frac{V_{a}}{c}\left(t_{1}\right)\right]^{2}-4 \cdot \frac{\beta_{t_{1}}}{2} \frac{a_{a}}{c}\left(t_{1}\right) \cdot \Delta t_{1}{ }^{(1)}\left(1-\beta_{t_{1}} \frac{V_{a}}{c}\left(t_{1}\right)\right) \\
& <=>\Delta=\left(1-\beta_{t_{1}} \frac{V_{a}}{c}\left(t_{1}\right)\right)\left(1-\beta_{t_{1}}\left(\frac{V_{a}}{c}\left(t_{1}\right)+\frac{a_{a}}{c}\left(t_{1}\right) \cdot 2 \Delta t_{1}{ }^{(1)}\right)\right) \\
& <=>\Delta=\left(1-\beta_{t_{1}} \frac{V_{a}}{c}\left(t_{1}\right)\right)\left(1-\beta_{t_{1}} \frac{V_{a}}{c}\left(t_{1}+2 \Delta t_{1}^{(1)}\right)\right) \\
& \Delta>0<=>1>\beta_{t_{1}} \frac{V_{a}}{c}\left(t_{1}+2 . \Delta t_{1}^{(1)}\right) \text { which is always true } \\
& =>\Delta t_{1}{ }^{(2)}=\frac{\left(1-\beta_{t_{1}} \frac{V_{a}}{c}\left(t_{1}\right)\right) \pm \sqrt{\left(1-\beta_{t_{1}} V_{a}\left(t_{1}\right)\right)\left(1-\beta_{t_{1}} \frac{V_{a}}{c}\left(t_{1}+2 \Delta t_{1}^{(1)}\right)\right)}}{\beta_{t_{1}} \frac{a_{a}}{c}\left(t_{1}\right)} \\
& <=>\Delta t_{1}^{(2)}=\frac{\left(1-\beta_{t_{1}} \frac{V_{a}}{c}\left(t_{1}\right)\right) \pm \sqrt{\left(1-\beta_{t_{1}} \frac{V_{a}}{c}\left(t_{1}\right)\right)\left(1-\beta_{t_{1}}\left(\frac{V_{a}}{c}\left(t_{1}\right)+\frac{a_{a}}{c}\left(t_{1}\right) \cdot 2 \Delta t_{1}^{(1)}\right)\right)}}{\beta_{t_{1}} \frac{a_{a}}{c}\left(t_{1}\right)} \\
& <=\Delta t_{1}^{(2)}=\frac{\left(1-\beta_{t_{1}} \frac{V_{a}}{c}\left(t_{1}\right)\right)}{\beta_{t_{1}} \frac{a_{a}}{c}\left(t_{1}\right)}\left[1 \pm \sqrt{\frac{1-\beta_{t_{1}}\left(\frac{V_{a}}{c}\left(t_{1}\right)+\frac{a_{a}}{c}\left(t_{1}\right) \cdot 2 \Delta t_{1}^{(1)}\right)}{1-\beta_{t_{1}} \frac{V_{a}}{c}\left(t_{1}\right)}}\right] \\
& <=>\Delta t_{1}{ }^{(2)}=\frac{\left(1-\beta_{t_{1}} V_{a}\left(t_{1}\right)\right)}{\beta_{t_{1}} \frac{a_{a}}{c}\left(t_{1}\right)}\left[1 \pm \sqrt{1-\frac{\beta_{t_{1}} \frac{a_{a}}{c}\left(t_{1}\right)}{1-\beta_{t_{1}} \frac{V_{a}}{c}\left(t_{1}\right)} \cdot 2 \Delta t_{1}^{(1)}}\right] \\
& <=>\Delta t_{1}{ }^{(2)} \approx \frac{\left(1-\beta_{t_{1}} \frac{V_{a}}{c}\left(t_{1}\right)\right)}{\beta_{t_{1}} \frac{a_{a}}{c}\left(t_{1}\right)}\left[1 \pm 1 \mp \frac{\beta_{t_{1}} \frac{a_{a}}{c}\left(t_{1}\right)}{1-\beta_{t_{1}} \frac{V_{a}}{c}\left(t_{1}\right)} \cdot \Delta t_{1}{ }^{(1)}\right] \\
& <=>\Delta t_{1}{ }^{(2)} \approx \mp \Delta t_{1}{ }^{(1)}+(1 \pm 1) \frac{\left(1-\beta_{t_{1}} \frac{V_{a}}{c}\left(t_{1}\right)\right)}{\beta_{t_{1}} \frac{a_{a}}{c}\left(t_{1}\right)} \\
& \Delta t_{1}{ }^{(2)} \approx \Delta t_{1}^{(1)} \equiv \frac{\beta_{t_{1}}}{c} \cdot \frac{x_{a}\left(t_{1}\right)-x_{c}\left(t_{1}\right)}{1-\beta_{t_{1}} \frac{V_{a}}{c}\left(t_{1}\right)} \\
& \text { or } \approx-\Delta t_{1}^{(1)}+2 \frac{\left(1-\beta_{t_{1}} \frac{V_{a}}{c}\left(t_{1}\right)\right)}{\beta_{t_{1}} a_{a}\left(t_{1}\right)}
\end{aligned}
$$


The position where $\mathrm{E}_{t_{1}}$ takes place in $\mathrm{K}$ is therefore $x_{a}\left(t_{1}+\Delta t_{1}\right)$ :

$$
\text { With } \Delta t_{1} \approx \Delta t_{1}{ }^{(1)}+\frac{\left[\frac{\beta_{t_{1} a} a}{2}\left(t_{1}\right)\right]}{1-\beta_{t_{1}} \frac{V a}{c}\left(t_{1}\right)} \Delta t_{1}^{(1)^{2}} \text {, and } \Delta t_{1}{ }^{(1)} \equiv \frac{\beta_{t_{1}}}{c} \cdot \frac{x_{a}\left(t_{1}\right)-x_{c}\left(t_{1}\right)}{1-\beta_{t_{1}} \frac{V a}{c}\left(t_{1}\right)}
$$

We have finally:

$$
\mathrm{E}_{t_{1}}=\left(c \cdot t_{C\left(t_{1}\right)}^{*}, x_{a, K_{1}^{*}}\right)_{K_{1}^{*}}=\left(c\left(t_{1}+\Delta t_{1}\right), x_{a}\left(t_{1}+\Delta t_{1}\right)\right)_{K}
$$

with $\Delta t_{1}=\frac{\beta_{t_{1}}}{c} \cdot\left(x_{a}\left(t_{1}+\Delta t_{1}\right)-x_{c}\left(t_{1}\right)\right)$, that we can call it the shift time : the time to wait after $t_{1}$ in order to have the event "the particle "a" arrives on the hyper plane of $K^{*}\left(t_{1}\right)$ ".

We can notice that:

- $\mathrm{E}_{t_{1}} \neq\left(\text { c. } t_{1}, \ldots\right)_{K}$

- $\boldsymbol{g}_{\boldsymbol{a}}: t_{i} \rightarrow \mathrm{E}_{t_{i}}=\left(c .\left(t_{i}+\Delta t_{i}\right), x_{a}\left(t_{i}+\Delta t_{i}\right)\right)_{K}$

We clearly see that $\mathrm{E}_{t_{1}}$ is parameterized by $t_{1}$ although it is not seen at this instant in $\mathrm{K}$ but at the instant $t=t_{1}+\Delta t_{1}$.

Another interesting point is that, at the $t_{1}$, the internal events that take place in $K^{*}\left(t_{1}\right)$ are not of the kind $\left(c\left(t_{1}\right), x_{a}\left(t_{1}\right)\right)_{K}$ but the "shifted" version $\left(c\left(t_{1}+\Delta t_{1}\right), x_{a}\left(t_{1}+\Delta t_{1}\right)\right)_{K}$. That is to say the internal events considered (spatio-temporal position of particle) will happen in the future (or the past, depending the position compared to the mass centre). The weird consequence (another one of Relativity...) is that the internal energy and so the mass, is relative to the future and the past of the material system (and also field as we will see below), in the point of view of $\mathrm{K}$.

\subsubsection{What is the difference of coordinates of the particle for infinitesimal interval dt, seen in $\mathrm{K}$ ?}

With the same reasoning, we have at the instant $t_{2}$ just after $t_{1}$ :

$$
\begin{gathered}
\mathrm{E}_{t_{2}}=\left(c \cdot t_{C\left(t_{2}\right)}^{*}, x_{a, K_{2}}\right)_{K_{2}^{*}}=\left(c\left(t_{2}+\Delta t_{2}\right), x_{a}\left(t_{2}+\Delta t_{2}\right)\right)_{K} \\
\text { With } \Delta t_{2} \approx \Delta t_{2}{ }^{(1)}+\frac{\left[\frac{\beta_{t_{2}} a_{a}}{2}\left(t_{2}\right)\right]}{1-\beta_{t_{2}} \frac{V a}{c}\left(t_{2}\right)} \Delta t_{2}{ }^{(1)^{2}} \text {, and } \Delta t_{2}{ }^{(1)} \equiv \frac{\beta_{t_{2}}}{c} \cdot \frac{x_{a}\left(t_{2}\right)-x_{c}\left(t_{2}\right)}{1-\beta_{t_{2}} \frac{V a}{c}\left(t_{2}\right)}
\end{gathered}
$$

So by doing the simple algebraic difference in $\mathrm{K}$, we have:

$$
\begin{gathered}
\mathrm{E}_{t_{2}}-\mathrm{E}_{t_{1}}=\left(c .\left(t_{2}+\Delta t_{2}\right), x_{a}\left(t_{2}+\Delta t_{2}\right)\right)_{K}-\left(c \cdot\left(t_{1}+\Delta t_{1}\right), x_{a}\left(t_{1}+\Delta t_{1}\right)\right)_{K} \\
=\left(c \cdot\left(t_{2}-t_{1}\right)+c\left(\Delta t_{2}-\Delta t_{1}\right),\left[x_{a}\right]_{t_{1}+\Delta t_{1}}^{t_{2}+\Delta t_{2}}\right)_{K}
\end{gathered}
$$

With $\left[x_{a}\right]_{t_{1}+\Delta t_{1}}^{t_{2}+\Delta t_{2}} \equiv x_{a}\left(t_{2}+\Delta t_{2}\right)-x_{a}\left(t_{1}+\Delta t_{1}\right)$ 
When $\left(t_{2}-t_{1}\right)$ tends to $\mathrm{dt}$ (no $2^{\text {nd }}$ degree), we have:

$$
\begin{aligned}
& \circ \beta_{t_{2}}=\beta_{t_{1}}+\left(\frac{d \beta_{t}}{d t}\right)_{t_{1}}\left(t_{2}-t_{1}\right) \\
& \circ \Delta t_{2}=\Delta t_{1}+\left(t_{2}-t_{1}\right)\left(\frac{d}{d t} \Delta t\right)_{t_{1}}
\end{aligned}
$$

With:

$$
\begin{aligned}
& \circ \Delta t_{1} \approx \Delta t_{1}{ }^{(1)}+\frac{\left[\frac{\beta_{1} a_{a}}{2}\left(t_{1}\right)\right]}{1-\beta_{t_{1}} \frac{V a}{c}\left(t_{1}\right)} \Delta t_{1}{ }^{(1)^{2}} \\
& \circ \quad \Delta t_{1}{ }^{(1)} \equiv \frac{\beta_{t_{1}}}{c} \cdot \frac{x_{a}\left(t_{1}\right)-x_{c}\left(t_{1}\right)}{1-\beta_{t_{1}} \frac{V a}{c}\left(t_{1}\right)} \\
& \circ\left(\frac{d}{d t} \Delta t_{1}\right)_{t_{1}}=\frac{d}{d t}\left(\Delta t_{1}{ }^{(1)}+\Delta t_{1}{ }^{(1)} \frac{1}{2 c} \frac{\beta_{t_{1} a_{a}}}{1-\frac{1}{c} \beta_{t_{1} V} V}\right)
\end{aligned}
$$

Moreover $\left[x_{a}\right]_{t_{1}+\Delta t_{1}}^{t_{2}+\Delta t_{2}}=x_{a}\left(t_{2}+\Delta t_{2}\right)-x_{a}\left(t_{1}+\Delta t_{1}\right)=x_{a}\left(t_{1}+\left(t_{2}-t_{1}\right)+\Delta t_{2}\right)-x_{a}\left(t_{1}+\Delta t_{1}\right)$

$=x_{a}\left(t_{1}+\left(t_{2}-t_{1}\right)+\Delta t_{2}\right)-x_{a}\left(t_{1}+\Delta t_{1}\right)$

$=x_{a}\left(t_{1}+\left(t_{2}-t_{1}\right)+\Delta t_{1}+\left(\frac{\mathrm{d} \Delta t}{d t}\right)_{t_{1}}\left(t_{2}-t_{1}\right)\right)-x_{a}\left(t_{1}+\Delta t_{1}\right)$

$=x_{a}\left(t_{1}+\Delta t_{1}+\left(t_{2}-t_{1}\right)\left[1+\left(\frac{\mathrm{d} \Delta t}{d t}\right)_{t_{1}}\right]\right)-x_{a}\left(t_{1}+\Delta t_{1}\right)$

$=x_{a}\left(t_{1}+\Delta t_{1}\right)+\left(\frac{\mathrm{d} x_{a}}{d t}\right)_{t_{1}+\Delta t_{1}}\left(t_{2}-t_{1}\right)\left[1+\left(\frac{\mathrm{d} \Delta t}{d t}\right)_{t_{1}}\right]-x_{a}\left(t_{1}+\Delta t_{1}\right)$

$\left[x_{a}\right]_{t_{1}+\Delta t_{1}}^{t_{2}+\Delta t_{2}}=\left(t_{2}-t_{1}\right) \cdot\left(\frac{\mathrm{d} x_{a}}{d t}\right)_{t_{1}+\Delta t_{1}} \cdot\left[1+\left(\frac{\mathrm{d} \Delta t}{d t}\right)_{t_{1}}\right]$

$=>\mathrm{E}_{t_{2}}-\mathrm{E}_{t_{1}}=\left(c \cdot\left(t_{2}-t_{1}\right)+c\left(\Delta t_{2}-\Delta t_{1}\right),\left[x_{a}\right]_{t_{1}+\Delta t_{1}}^{t_{2}+\Delta t_{2}}\right)_{K}$

$=\left(c \cdot\left(t_{2}-t_{1}\right)+c\left(\frac{\mathrm{d} \Delta t}{d t}\right)_{t_{1}}\left(t_{2}-t_{1}\right),\left(t_{2}-t_{1}\right) \cdot\left(\frac{\mathrm{d} x_{a}}{d t}\right)_{t_{1}+\Delta t_{1}} \cdot\left(1+\left(\frac{\mathrm{d} \Delta t}{d t}\right)_{t_{1}}\right)\right)_{K}$

$=c \cdot\left(t_{2}-t_{1}\right)\left(1+\left(\frac{\mathrm{d} \Delta t}{d t}\right)_{t_{1}}\right) \cdot\left(1, \frac{1}{c}\left(\frac{\mathrm{d} x_{a}}{d t}\right)_{t_{1}+\Delta t_{1}}\right)_{K}$

$=>\mathrm{E}_{t_{2}}-\mathrm{E}_{t_{1}}=c \cdot\left(t_{2}-t_{1}\right)\left(1+\left(\frac{\mathrm{d} \Delta t}{d t}\right)_{t_{1}}\right) \cdot\left(1, \frac{1}{c}\left(\frac{\mathrm{d} x_{a}}{d t}\right)_{t_{1}+\Delta t_{1}}\right)_{K}$

With:

$$
\begin{aligned}
& \circ \Delta t_{1} \approx \Delta t_{1}{ }^{(1)}+\frac{\left[\frac{\beta_{t_{1} a_{a}}}{2}\left(t_{1}\right)\right]}{1-\beta_{t_{1}} \frac{V a}{c}\left(t_{1}\right)} \Delta t_{1}{ }^{(1)^{2}} \\
& \circ \quad \Delta t_{1}{ }^{(1)} \equiv \frac{\beta_{t_{1}}}{c} \cdot \frac{x_{a}\left(t_{1}\right)-x_{c}\left(t_{1}\right)}{1-\beta_{t_{1}} \frac{V a}{c}\left(t_{1}\right)}
\end{aligned}
$$




$$
\circ\left(\frac{d}{d t} \Delta t_{1}\right)_{t_{1}}=\frac{d}{d t}\left(\Delta t_{1}{ }^{(1)}+\Delta t_{1}{ }^{(1)^{2}} \frac{1}{2 c} \frac{\beta_{t_{1} a_{a}}}{1-\frac{1}{c} \beta_{t_{1}} v_{a}}\right)
$$

We can use this difference of events in order to calculate the speed of a particle "a" with these 2 events, we have:

$$
\left(\frac{x_{\mathrm{E}_{t_{2}}}-x_{\mathrm{E}_{t_{1}}}}{t_{\mathrm{E}_{t_{2}}}-t_{\mathrm{E}_{t_{1}}}}\right)_{t_{1}, K}=V_{a / K}\left(t_{1}+\Delta t_{1}\right)
$$

The speed associated to the 2 events $\mathrm{E}_{t_{2}} \& \mathrm{E}_{t_{1}}$ is actually different than the one associated to the speed measured by $\mathrm{K}$ in the standard way. It is of course different to study in $\mathrm{K} 2$ events observed at the instant $t_{1} \& t_{1}+d t$ than the 2 others at $t_{1}+\Delta t_{1} \& t_{2}+\Delta t_{2}$.

We recover the standard speed at a given time $t$ when the particle is sufficiently close to the mass centre $\mathrm{C}=\Delta t_{1}{ }^{(1)} \approx 0$.

\subsubsection{What is the difference of coordinates of the particle for infinitesimal interval dt, seen in $\mathrm{K}^{*}$}

The first event is:

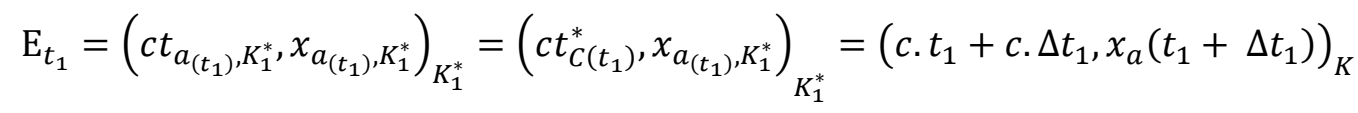

Remark: we use the expression $x_{a_{\left(t_{1}\right)}, K_{1}^{*}}$ as we have explained above that the events in $K^{*}\left(t_{1}\right)$ are parameterized via the map $\mathbf{g}_{\mathbf{a}}$.

According to Lorentz $\left\{\begin{array}{l}\left(c . t_{1}+c . \Delta t_{1}\right)-c \cdot t_{1}=\gamma_{t_{i}} \cdot\left(\mathrm{c}\left(t_{a_{\left(t_{1}\right)}, K_{1}^{*}}-t_{C\left(t_{1}\right)}^{*}\right)+\beta_{t_{1}} \cdot x_{a_{\left(t_{1}\right)}, K_{1}^{*}}\right) \\ x_{a}\left(t_{1}+\Delta t_{1}\right)-x_{c}\left(t_{1}\right)=\gamma_{t_{1}} \cdot\left(x_{a_{\left(t_{1}\right)}, K_{1}^{*}}+\beta_{t_{1}} \cdot\left(t_{a_{\left(t_{1}\right)}, K_{1}^{*}}-t_{C\left(t_{1}\right)}^{*}\right)\right)\end{array}\right.$

$$
\begin{aligned}
& <=>
\end{aligned}
$$

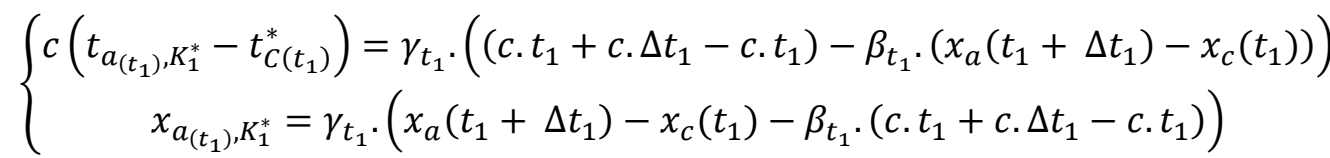

$$
\begin{aligned}
& \Leftrightarrow=\left\{\begin{array}{c}
t_{a_{\left(t_{1}\right)}, K_{1}^{*}}=\gamma_{t_{1} \cdot} \cdot\left(\beta_{t_{1} \cdot}\left(x_{a}\left(t_{1}+\Delta t_{1}\right)-x_{c}\left(t_{1}\right)\right)-\beta_{t_{1}} \cdot\left(x_{a}\left(t_{1}+\Delta t_{1}\right)-x_{c}\left(t_{1}\right)\right)\right) \\
x_{a_{\left(t_{1}\right)}, K_{1}^{*}}=\gamma_{t_{1}} \cdot\left(x_{a}\left(t_{1}+\Delta t_{1}\right)-x_{c}\left(t_{1}\right)-\beta_{t_{1}} \cdot c \cdot \Delta t_{1}\right)
\end{array}\right. \\
& <=\left\{\begin{array}{c}
c\left(t_{a_{\left(t_{1}\right)}, K_{1}^{*}}-t_{C\left(t_{1}\right)}^{*}\right)=0=>\text { as it should } \\
x_{a_{\left(t_{1}\right)}, K_{1}^{*}}=\gamma_{t_{1}} \cdot\left(x_{a}\left(t_{1}+\Delta t_{1}\right)-x_{c}\left(t_{1}\right)-\beta_{t_{1}} \cdot \beta_{t_{1}} \cdot x_{a}\left(t_{1}+\Delta t_{1}\right)-x_{c}\left(t_{1}\right)\right)
\end{array}\right. \\
& k=\left\{\begin{array}{c}
\left(t_{a_{\left(t_{1}\right)}, K_{1}^{*}}-t_{C\left(t_{1}\right)}^{*}\right)=0 \\
x_{a_{\left(t_{1}\right)}, K_{1}^{*}}=\frac{x_{a}\left(t_{1}+\Delta t_{1}\right)-x_{c}\left(t_{1}\right)}{\gamma_{t_{1}}}=\frac{c \Delta t_{1}}{\beta_{t_{1}} \cdot \gamma_{t_{1}}}
\end{array}\right.
\end{aligned}
$$

We use $\Delta t_{1}=\frac{\beta_{t_{1}}}{c} \cdot\left(x_{a}\left(t_{1}+\Delta t_{1}\right)-x_{c}\left(t_{1}\right)\right)$

The second event is:

$$
\mathrm{E}_{2}=\left(c t_{a_{\left(t_{2}\right)}, K_{2}^{*}}, x_{a_{\left(t_{2}\right)}, K_{2}^{*}}\right)_{K_{1}^{*}}=\left(c \cdot t_{C\left(t_{2}\right)}^{*}, x_{a_{\left(t_{2}\right)}, K_{2}^{*}}\right)_{K_{2}^{*}}=\left(c \cdot\left(t_{2}+\Delta t_{2}\right), x_{a}\left(t_{2}+\Delta t_{2}\right)\right)_{K}
$$


But, in point of view of $K_{1}^{*}$ we have also

$$
\mathrm{E}_{2}=\left(c \cdot t_{a_{\left(t_{2}\right)}, K_{1}^{*}}, x_{a_{\left(t_{2}\right)}, K_{1}^{*}}\right)_{K_{1}^{*}}=\left(c . t_{2}+\beta_{t_{2}} \cdot\left(x_{a}\left(t_{2}\right)-x_{c}\left(t_{2}\right)\right), x_{a}\left(t_{2}\right)\right)_{K}
$$

\section{Remark:}

- In the notation $t_{a_{\left(t_{2}\right), K_{1}^{*}}}$ we have to note the small change: this is the event in the hyperperplane of $K^{*}\left(t_{2}\right)$ parametrized at $t_{2}$ but seen by an observatory in the frame $K^{*}\left(t_{1}\right)$.

$\circ \quad$ c. $t_{a_{\left(t_{2}\right)}, K_{1}^{*}} \neq t_{C\left(t_{1}\right)}^{*}$ a priori

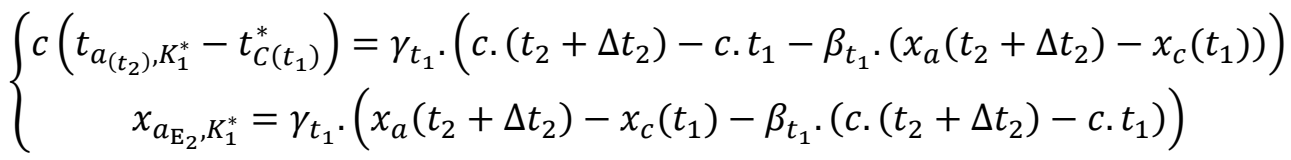

$$
\begin{aligned}
& c\left(t_{a_{\left(t_{2}\right)}, K_{1}^{*}}-t_{C\left(t_{1}\right)}^{*}\right)=\gamma_{t_{1}} \cdot\left(c .\left(t_{2}+\Delta t_{2}\right)-c . t_{1}-\beta_{t_{1}} \cdot\left(x_{a}\left(t_{2}+\Delta t_{2}\right)-x_{c}\left(t_{2}\right)-x_{c}\left(t_{1}\right)+x_{c}\left(t_{2}\right)\right)\right) \\
& =\gamma_{t_{1}} \cdot\left(c \cdot\left(t_{2}+\Delta t_{2}\right)-c \cdot t_{1}-c \frac{\Delta t_{2}}{\beta_{t_{2}}} \beta_{t_{1}}-\beta_{t_{1}}\left(-x_{c}\left(t_{1}\right)+x_{c}\left(t_{2}\right)\right)\right) \\
& =\gamma_{t_{1}} \cdot\left(c \cdot t_{2}+c \cdot \Delta t_{2}-c \cdot t_{1}-c \frac{\Delta t_{2}}{\beta_{t_{2}}} \beta_{t_{1}}-\left(t_{2}-t_{1}\right) \frac{\beta_{t_{1}}}{c} V_{c}\left(t_{1}\right)\right) \\
& =\gamma_{t_{1}} \cdot\left(c \cdot\left(t_{2}-t_{1}\right)+c \cdot \Delta t_{2}-c \frac{\Delta t_{2}}{\beta_{t_{2}}} \beta_{t_{1}}-\left(t_{2}-t_{1}\right) \beta_{t_{1}}^{2}\right) \\
& =\gamma_{t_{1}} \cdot c \cdot\left(\Delta t_{2}\left(1-\frac{\beta_{t_{1}}}{\beta_{t_{2}}}\right)+\left(t_{2}-t_{1}\right)\left(1-\frac{\beta_{t_{1}}^{2}}{c}\right)\right) \\
& \text { because } \Delta t_{2}=\Delta t_{1}+\left(t_{2}-t_{1}\right)\left(\frac{d}{d t} \Delta t_{1}\right)_{t_{1}} \\
& \text { and } \Delta t_{2}=\frac{\beta_{t_{2}}}{c} \cdot\left(x_{a}\left(t_{2}+\Delta t_{2}\right)-x_{c}\left(t_{2}\right)\right) \\
& =\gamma_{t_{1}} \cdot c \cdot\left(\Delta t_{2}\left(1-\frac{\beta_{t_{1}}}{\beta_{t_{1}}+\left(\frac{d \beta_{t}}{d t}\right)_{t_{1}}\left(t_{2}-t_{1}\right)}\right)+\frac{\left(t_{2}-t_{1}\right)}{\gamma_{t_{1}}{ }^{2}}\right) \\
& \frac{1}{\beta_{t_{1}}+\left(\frac{d \beta_{t}}{d t}\right)_{t_{1}}\left(t_{2}-t_{1}\right)}=\frac{1}{\beta_{t_{1}}}+\left(\frac{d}{d X}\left(\frac{1}{X}\right)\right)_{X=\beta_{t_{1}}}\left(\frac{d \beta_{t}}{d t}\right)_{t_{1}}\left(t_{2}-t_{1}\right)=\frac{1}{\beta_{t_{1}}}+\left(\frac{-1}{X^{2}}\right)_{X=\beta_{t_{1}}}\left(\frac{d \beta_{t}}{d t}\right)_{t_{1}}\left(t_{2}-t_{1}\right) \\
& =\frac{1}{\beta_{t_{1}}}-\frac{1}{\beta_{t_{1}}{ }^{2}}\left(\frac{d \beta_{t}}{d t}\right)_{t_{1}}\left(t_{2}-t_{1}\right) \\
& c\left(t_{a_{\left(t_{2}\right)}, K_{1}^{*}}-t_{C\left(t_{1}\right)}^{*}\right)=\gamma_{t_{1}} \cdot c \cdot\left(\Delta t_{2}\left(1-\beta_{t_{1}}\left(\frac{1}{\beta_{t_{1}}}-\frac{1}{\beta_{t_{1}}{ }^{2}}\left(\frac{d \beta_{t}}{d t}\right)_{t_{1}}\left(t_{2}-t_{1}\right)\right)\right)+\frac{\left(t_{2}-t_{1}\right)}{\gamma_{t_{1}}{ }^{2}}\right) \\
& =\gamma_{t_{1}} \cdot c \cdot\left(\Delta t_{2}\left(\frac{1}{\beta_{t_{1}}}\left(\frac{d \beta_{t}}{d t}\right)_{t_{1}}\left(t_{2}-t_{1}\right)\right)+\frac{\left(t_{2}-t_{1}\right)}{\gamma_{t_{1}}{ }^{2}}\right) \\
& =\gamma_{t_{1}} \cdot c \cdot\left(\Delta t_{2} \frac{1}{\beta_{t_{1}}}\left(\frac{d \beta_{t}}{d t}\right)_{t_{1}}\left(t_{2}-t_{1}\right)+\frac{\left(t_{2}-t_{1}\right)}{\gamma_{t_{1}}{ }^{2}}\right) \\
& =c \cdot \frac{\left(t_{2}-t_{1}\right)}{\gamma_{t_{1}}}+\gamma_{t_{1}} \cdot c \cdot \Delta t_{2} \frac{1}{\beta_{t_{1}}}\left(\frac{d \beta_{t}}{d t}\right)_{t_{1}}\left(t_{2}-t_{1}\right) \\
& =c . \frac{\left(t_{2}-t_{1}\right)}{\gamma_{t_{1}}}+\gamma_{t_{1}} \cdot c \cdot\left(\Delta t_{1}+\left(t_{2}-t_{1}\right)\left(\frac{d}{d t} \Delta t_{1}\right)_{t_{1}}\right) \frac{1}{\beta_{t_{1}}}\left(\frac{d \beta_{t}}{d t}\right)_{t_{1}}\left(t_{2}-t_{1}\right)
\end{aligned}
$$




$$
c\left(t_{a_{\left(t_{2}\right)}, K_{1}^{*}}-t_{C\left(t_{1}\right)}^{*}\right)_{K * \text { not Galilean }}=c \cdot \frac{\left(t_{2}-t_{1}\right)}{\gamma_{t_{1}}}+\gamma_{t_{1}} \cdot \frac{c \cdot \Delta t_{1}}{\beta_{t_{1}}}\left(\frac{d \beta_{t}}{d t}\right)_{t_{1}}\left(t_{2}-t_{1}\right)
$$

But, since we use at each time a local Galilean frame, there are non acceleration for this frame (the condition for the use of Lorentz transformation): $\left(\frac{d \beta_{t}}{d t}\right)_{t_{1}, \text { Galilean }} \equiv 0$

$$
\begin{aligned}
& c\left(t_{a_{\left(t_{2}\right)}, K_{1}^{*}}-t_{C\left(t_{1}\right)}^{*}\right)=c \cdot \frac{\left(t_{2}-t_{1}\right)}{\gamma_{t_{1}}} \\
& x_{a_{\left(t_{2}\right)}, K_{1}^{*}}=\gamma_{t_{1}} \cdot\left(x_{a}\left(t_{2}+\Delta t_{2}\right)-x_{c}\left(t_{1}\right)-\beta_{t_{1}} \cdot\left(c .\left(t_{2}+\Delta t_{2}\right)-c \cdot t_{1}\right)\right) \\
& =\gamma_{t_{1}} \cdot\left(x_{a}\left(t_{1}+\left(t_{2}-t_{1}\right)+\Delta t_{2}\right)-x_{c}\left(t_{1}\right)-\beta_{t_{1}} \cdot\left(c \cdot \Delta t_{2}+c \cdot\left(t_{2}-t_{1}\right)\right)\right) \\
& =\gamma_{t_{1}} \cdot\left(x_{a}\left(t_{1}+\left(t_{2}-t_{1}\right)+\Delta t_{1}+\left(t_{2}-t_{1}\right)\left(\frac{d}{d t} \Delta t_{1}\right)_{t_{1}}\right)-x_{c}\left(t_{1}\right)-\beta_{t_{1}} \cdot c\left(\Delta t_{1}+\left(t_{2}-t_{1}\right)\left(\frac{d}{d t} \Delta t_{1}\right)_{t_{1}}\right)-\beta_{t_{1}} \cdot\left(c \cdot\left(t_{2}-t_{1}\right)\right)\right) \\
& =\gamma_{t_{1}} \cdot\left[x_{a}\left(t_{1}+\Delta t_{1}+\left(t_{2}-t_{1}\right)\left(1+\left(\frac{d}{d t} \Delta t_{1}\right) t_{t_{1}}\right)\right)-x_{c}\left(t_{1}\right)-\beta_{t_{1}} \cdot c\left(\Delta t_{1}+\left(t_{2}-t_{1}\right)\left(1+\left(\frac{d}{d t} \Delta t_{1}\right)\right)\right)\right] \\
& =\gamma_{t_{1}} \cdot\left[x_{a}\left(t_{1}+\Delta t_{1}\right)+\left(\frac{d}{d t} x_{a}\right)_{t_{1+\Delta t_{1}}} \cdot\left(t_{2}-t_{1}\right)\left(1+\left(\frac{d}{d t} \Delta t_{1}\right)_{t_{1}}\right)-x_{c}\left(t_{1}\right)-\beta_{t_{1}} \cdot c\left(\Delta t_{1}+\left(t_{2}-t_{1}\right)\left(1+\left(\frac{d}{d t} \Delta t_{1}\right)\right)\right)\right] \\
& =\gamma_{t_{1}} \cdot\left[x_{a}\left(t_{1}+\Delta t_{1}\right)-x_{c}\left(t_{1}\right)+V_{a}\left(t_{1}+\Delta t_{1}\right) \cdot\left(t_{2}-t_{1}\right)\left(1+\left(\frac{d}{d t} \Delta t_{1}\right)_{t_{1}}\right)-\beta_{t_{1}} \cdot c \Delta t_{1}-\beta_{t_{1}} \cdot c\left(t_{2}-t_{1}\right)\left(1+\left(\frac{d}{d t} \Delta t_{1}\right){ }_{t_{1}}\right)\right] \\
& =\gamma_{t_{1}} \cdot\left(c \frac{\Delta t_{1}}{\beta_{t_{1}}}-\beta_{t_{1}} \cdot c \Delta t_{1}+\left(t_{2}-t_{1}\right) \cdot\left(1+\left(\frac{d}{d t} \Delta t_{1}\right)_{t_{1}}\right)\left[V_{a}\left(t_{1}+\Delta t_{1}\right)-\beta_{t_{1}} \cdot c\right]\right) \\
& =\gamma_{t_{1}} \cdot\left(c \Delta t_{1}\left(\frac{1-\beta_{t_{1}}^{2}}{\beta_{t_{1}}}\right)+\left(t_{2}-t_{1}\right) \cdot\left(1+\left(\frac{d}{d t} \Delta t_{1}\right)_{t_{1}}\right)\left[V_{a}\left(t_{1}+\Delta t_{1}\right)-\beta_{t_{1}} \cdot c\right]\right) \\
& =\gamma_{t_{1}} \cdot\left(\frac{c \Delta t_{1}}{\gamma_{t_{1}}{ }^{2}} \frac{1}{\beta_{t_{1}}}+\left(t_{2}-t_{1}\right) \cdot\left(1+\left(\frac{d}{d t} \Delta t_{1}\right)_{t_{1}}\right)\left[V_{a}\left(t_{1}+\Delta t_{1}\right)-\beta_{t_{1}} \cdot c\right]\right) \\
& =\gamma_{t_{1}}\left(t_{2}-t_{1}\right) \cdot\left(1+\left(\frac{d}{d t} \Delta t_{1}\right)_{t_{1}}\right) \cdot c\left(\frac{V_{a}}{c}\left(t_{1}+\Delta t_{1}\right)-\beta_{t_{1}}\right) \\
& \text { Because } x_{a_{\left(t_{1}\right)}, K_{1}^{*}}=\frac{x_{a}\left(t_{1}+\Delta t_{1}\right)-x_{c}\left(t_{1}\right)}{\gamma_{t_{1}}}=\frac{c \Delta t_{1}}{\beta_{t_{1}} \cdot \gamma_{t_{1}}}
\end{aligned}
$$

We have:

$$
\begin{aligned}
x_{a_{\left(t_{2}\right)}, K_{1}^{*}}-x_{a_{\left(t_{1}\right)}, K_{1}^{*}}= & \gamma_{t_{1}}\left(t_{2}-t_{1}\right) \cdot\left(1+\left(\frac{d}{d t} \Delta t_{1}\right){ }_{t_{1}}\right) \cdot c\left(\frac{V_{a}}{c}\left(t_{1}+\Delta t_{1}\right)-\beta_{t_{1}}\right) \\
& c\left(t_{a_{\left(t_{2}\right), K_{1}^{*}}}-t_{C\left(t_{1}\right)}^{*}\right)=c \cdot \frac{\left(t_{2}-t_{1}\right)}{\gamma_{t_{1}}}
\end{aligned}
$$

Thus

$$
\begin{aligned}
\Rightarrow \mathrm{E}_{2}-\mathrm{E}_{1} & =\left(c\left(t_{a_{\left(t_{2}\right)}, K_{1}^{*}}-t_{C\left(t_{1}\right)}^{*}\right), x_{a_{\left(t_{2}\right)}, K_{1}^{*}}-x_{a_{\left(t_{1}\right)}, K_{1}^{*}}\right)_{\mathrm{K}_{1}^{*}} \\
& =\text { c. }\left(t_{2}-t_{1}\right)\left(\frac{1}{\gamma_{t_{1}}},\left(1+\left(\frac{d}{d t} \Delta t_{1}\right)_{t_{1}}\right) \cdot\left(\frac{V_{a}}{c}\left(t_{1}+\Delta t_{1}\right)-\beta_{t_{1}}\right)\right)_{\mathrm{K}_{1}^{*}}
\end{aligned}
$$


2.7.5. What is the expression of the speed in $\mathrm{K}$ and $\mathrm{K}^{*}$ and what are their relation (velocity addition formula)?

Using the expression above, we calculate different speed for different frame.

- Relative to the internal frame $K^{*}\left(t_{1}\right)$

$$
\begin{array}{r}
V_{a / K_{1}^{*}}\left(t_{1}^{*}\left(t_{1}\right)\right) \equiv V_{a / K_{1}^{*}}\left(\int_{0}^{t_{1}} \frac{d t}{\gamma_{t}}\right) \equiv \frac{x_{a_{\left(t_{2}\right)}, K_{1}^{*}}-x_{a_{\left(t_{1}\right), K_{1}^{*}}}}{t_{a_{\left(t_{2}\right)}, K_{1}^{*}}-t_{a_{\left(t_{1}\right)}, K_{1}^{*}}} \\
=\frac{\gamma_{t_{1}}\left(t_{2}-t_{1}\right) \cdot\left(1+\left(\frac{d}{d t} \Delta t_{1}\right)_{t_{1}}\right) \cdot c\left[\frac{V_{a / K}}{c}\left(t_{1}+\Delta t_{1}\right)-\beta_{t_{1}}\right]}{c \cdot \frac{\left(t_{2}-t_{1}\right)}{\gamma_{t_{1}}}} \\
\Leftrightarrow V_{a / K_{1}^{*}}\left(t_{1}^{*}\left(t_{1}\right)\right)=\gamma_{t_{1}}{ }^{2}\left(1+\left(\frac{d}{d t} \Delta t\right)_{t_{1}}\right) \cdot\left(V_{a / K}\left(t_{1}+\Delta t_{1}\right)-V_{C / K}\left(t_{1}\right)\right)
\end{array}
$$

But the "shift time" is:

$$
\begin{aligned}
& \Delta t_{1}=\frac{\beta_{t_{1}}}{c} \cdot\left(x_{a}\left(t_{1}+\Delta t_{1}\right)-x_{c}\left(t_{1}\right)\right) \\
& <=>\Delta t=\frac{\beta_{t}}{c} \cdot\left(x_{a}(t+\Delta t)-x_{c}(t)\right) \\
& =>\frac{d \Delta t}{d t}=\frac{\beta_{t}}{c} \cdot\left(\frac{d t+\Delta t}{d t}\left(\frac{d x_{a}}{d t}\right)_{(t+\Delta t)}-\frac{d}{d t} x_{c}(t)\right) \\
& <=>\frac{d \Delta t}{d t}=\frac{\beta_{t}}{c} \cdot\left(\left(1+\frac{d \Delta t}{d t}\right)\left(\frac{d x_{a}}{d t}\right)_{(t+\Delta t)}-\frac{d}{d t} x_{c}(t)\right) \\
& <=>\frac{d \Delta t}{d t}\left(1-\frac{\beta_{t}}{c}\left(\frac{d x_{a}}{d t}\right)_{(t+\Delta t)}\right)=\frac{\beta_{t}}{c} \cdot\left(\left(\frac{d x_{a}}{d t}\right)_{(t+\Delta t)}-\frac{d}{d t} x_{c}(t)\right) \\
& <=>\frac{d \Delta t}{d t}=\frac{\frac{\beta_{t}}{c} \cdot\left(\left(\frac{d x_{a}}{d t}\right)_{(t+\Delta t)}-\frac{d}{d t} x_{c}(t)\right)}{1-\frac{\beta_{t}}{c}\left(\frac{d x_{a}}{d t}\right)_{(t+\Delta t)}} \\
& =>1+\frac{d \Delta t}{d t}=\frac{1-\frac{\beta_{t}}{c}\left(\frac{d x_{a}}{d t}\right)_{(t+\Delta t)}+\frac{\beta_{t}}{c} \cdot\left(\left(\frac{d x_{a}}{d t}\right)_{(t+\Delta t)}-\frac{d}{d t} x_{c}(t)\right)}{1-\frac{\beta_{t}}{c}\left(\frac{d x_{a}}{d t}\right)_{(t+\Delta t)}} \\
& =\frac{1-\frac{\beta_{t}{ }^{2}}{c^{2}}}{1-\frac{\beta_{t}}{c}\left(\frac{d x_{a}}{d t}\right)_{(t+\Delta t)}}=\frac{1}{\gamma_{t_{1}}{ }^{2}} \frac{1}{1-\frac{\beta_{t}}{c}\left(\frac{d x_{a}}{d t}\right)_{(t+\Delta t)}} \\
& =>V_{a / K_{1}^{*}}\left(t_{1}^{*}\left(t_{1}\right)\right)=\gamma_{t_{1}}{ }^{2}\left(1+\left(\frac{d}{d t} \Delta t_{1}\right)_{t_{1}}\right) \cdot\left(V_{a / K}\left(t_{1}+\Delta t_{1}\right)-V_{C / K}\left(t_{1}\right)\right) \\
& =\frac{V_{a / K}\left(t_{1}+\Delta t_{1}\right)-V_{C / K}\left(t_{1}\right)}{1-\frac{V_{C / K}\left(t_{1}\right)}{c} \frac{V_{a / K}}{c}\left(t_{1}+\Delta t_{1}\right)}
\end{aligned}
$$




$$
\begin{aligned}
& =>V_{a / K_{1}^{*}}\left(t_{1}^{*}\left(t_{1}\right)\right)=\frac{V_{a / K}\left(t_{1}+\Delta t_{1}\right)-V_{C / K}\left(t_{1}\right)}{1-\frac{V_{C / K}\left(t_{1}\right)}{c} \frac{V_{a / K}\left(t_{1}+\Delta t_{1}\right)}{c}} \\
& \circ \Delta t_{1} \approx \Delta t_{1}{ }^{(1)}+\frac{\left[\frac{\beta_{t_{1} a_{a}}}{2}\left(t_{1}\right)\right]}{1-\beta_{t_{1}} \frac{V_{a}}{c}\left(t_{1}\right)} \Delta t_{1}{ }^{(1)^{2}} \\
& \text { ○ } \Delta t_{1}{ }^{(1)} \equiv \frac{\beta_{t_{1}}}{c} \cdot \frac{x_{a}\left(t_{1}\right)-x_{c}\left(t_{1}\right)}{1-\beta_{t_{1}} \frac{V_{a}}{c}\left(t_{1}\right)}
\end{aligned}
$$

We recover the Einstein-Poincaré formula when the system is closed to its center of mass $\left(\Delta t_{1} \approx\right.$ 0 ) or otherwise for particles without acceleration.

We have also

$$
\begin{gathered}
V_{a / K}\left(t_{1}+\Delta t_{1}\right)=V_{a / K_{1}^{*}}\left(t_{1}^{*}\left(t_{1}\right)\right)\left(1-\frac{V_{C / K}\left(t_{1}\right)}{c} \frac{V_{a / K}\left(t_{1}+\Delta t_{1}\right)}{c}\right)+V_{C / K}\left(t_{1}\right) \\
V_{a / K}\left(t_{1}+\Delta t_{1}\right)\left(1+\frac{V_{C / K}\left(t_{1}\right)}{c} \frac{V_{a / K_{1}^{*}}\left(t_{1}^{*}\left(t_{1}\right)\right)}{c}\right)=V_{a / K_{1}^{*}}\left(t_{1}^{*}\left(t_{1}\right)\right)+V_{C / K}\left(t_{1}\right) \\
=>V_{a / K}\left(t_{1}+\Delta t_{1}\right)=\frac{V_{a / K_{1}^{*}}\left(t_{1}^{*}\left(t_{1}\right)\right)+V_{C / K}\left(t_{1}\right)}{1+\frac{V_{C / K}\left(t_{1}\right)}{c} \frac{V_{a / K_{1}^{*}}\left(t_{1}^{*}\left(t_{1}\right)\right)}{c}}
\end{gathered}
$$

In the case of an accelerated particle in $\mathrm{K}$, we have:

$$
\begin{aligned}
\left(\frac{d x_{a}}{d t}\right)_{\left(t_{1}+\Delta t_{1}\right)} \approx\left(\frac{d x_{a}}{d t}\right)_{\left(t_{1}\right)}+\left(\frac{d^{2} x_{a}}{d t^{2}}\right)_{\left(t_{1}\right)} \Delta t_{1} \\
=>V_{a / K_{1}^{*}}\left(t_{1}^{*}\left(t_{1}\right)\right) \approx \frac{V_{a / K}\left(t_{1}\right)+\left(\frac{d^{2} x_{a}}{d t^{2}}\right)_{\left(t_{1}\right)} \Delta t_{1}-V_{C / K}\left(t_{1}\right)}{1-\frac{\beta_{t_{1}}}{c}\left(V_{a / K}\left(t_{1}\right)+\left(\frac{d^{2} x_{a}}{d t^{2}}\right)_{\left(t_{1}\right)} \Delta t_{1}\right)}
\end{aligned}
$$

We can also check that if one of the internal particles has the speed c, the apparent speed is again the invariant speed $\mathrm{c}$.

$$
V_{a / K_{1}^{*}}\left(t_{1}^{*}\left(t_{1}\right)\right)=\frac{c-V_{C}\left(t_{1}\right)}{1-\frac{\beta_{t_{1}}}{c} c}=c \frac{1-\beta_{t_{1}}}{1-\beta_{t_{1}}}=c
$$

- A second modified velocity addition formula

$$
\text { Since } \frac{x_{a_{\left(t_{2}\right)}, K_{1}^{*}-x_{a_{\left(t_{1}\right)}, K_{1}^{*}}}}{t_{2}-t_{1}}=\gamma_{t_{1}} \cdot\left(1+\left(\frac{d}{d t} \Delta t\right)_{t_{1}}\right) \cdot\left(V_{a}\left(t_{1}+\Delta t_{1}\right)-V_{C}\left(t_{1}\right)\right)
$$

With the same reasoning we have

$$
\frac{x_{a_{\left(t_{2}\right)}, K_{1}^{*}}-x_{a_{\left(t_{1}\right)}, K_{1}^{*}}}{t_{2}-t_{1}}=\frac{1}{\gamma_{t_{1}}} \frac{V_{a}\left(t_{1}+\Delta t_{1}\right)-V_{C}\left(t_{1}\right)}{1-\frac{\beta_{t_{1}}}{c} V_{a}\left(t_{1}+\Delta t_{1}\right)}
$$

With 
$\circ \Delta t_{1} \approx \Delta t_{1}{ }^{(1)}+\frac{\left[\frac{\beta_{t_{1}} a_{a}}{2}\left(t_{1}\right)\right]}{1-\beta_{t_{1}} \frac{V a}{c}\left(t_{1}\right)} \Delta t_{1}{ }^{(1)^{2}}$
$\circ \Delta t_{1}{ }^{(1)} \equiv \frac{\beta_{t_{1}}}{c} \cdot \frac{x_{a}\left(t_{1}\right)-x_{c}\left(t_{1}\right)}{1-\beta_{t_{1}} \frac{V a}{c}\left(t_{1}\right)}$

2.7.6. Conclusion about the proof

We can conclude that although during the proof we use a particular duration of time $d t_{1}=\gamma d t^{*}$, it is well defined as I try to convince the reader in this paragraph 2.6. We should carefully take care to the events implied by this way of reasoning. 


\section{Free field}

\subsection{The proof for a field}

Now, I will repeat the same method for a field theory (a scalar field $\varphi$ for simplify), and again:

The important point to keep in mind is that we are not considering the variation of the internal degree of freedom $\varphi^{*}$ :

- relative to the internal time $t^{*}$ of $\mathrm{K}^{*}: \frac{\partial \varphi^{*}}{\partial t^{*}}$;

- but instead relative to time $\mathbf{t}$ of $\mathrm{K}: \frac{\partial \varphi^{*}}{\partial t}$.

So without comments, we have successively:

$$
\begin{gathered}
S[\{\varphi(x, t)\}]=\frac{1}{c} \iiint \int \Lambda\left(\varphi, \frac{\partial \varphi}{\partial \boldsymbol{r}}, \frac{\partial \varphi}{\partial t}\right) d \Omega \\
=\frac{1}{c} \iiint \int \Lambda^{*}\left(\varphi^{*}, \frac{\partial \varphi^{*}}{\partial \boldsymbol{r}^{*}}, \frac{\partial \varphi^{*}}{\partial t^{*}}\right) d \Omega^{*}=\int\left[\iiint \Lambda^{*}\left(\varphi^{*}, \frac{\partial \varphi^{*}}{\partial \boldsymbol{r}^{*}}, \gamma\left(\boldsymbol{V}_{\mathrm{C}}\right) \frac{\partial \varphi^{*}}{\partial t}\right) d V^{*}\right] d t^{*} \\
=\int\left[\iiint \Lambda^{*}\left(\varphi^{*}, \frac{\partial \varphi^{*}}{\partial \boldsymbol{r}^{*}}, \gamma\left(V_{\mathrm{C}}\right) \frac{\partial \varphi^{*}}{\partial t}\right) d V^{*}\right] \frac{d t}{\gamma\left(\boldsymbol{V}_{\mathrm{C}}\right)} \\
=\int\left[\iint \frac{\Lambda^{*}\left(\varphi^{*}, \frac{\partial \varphi^{*}}{\partial \boldsymbol{r}^{*}}, \gamma\left(V_{\mathrm{C}}\right) \frac{\partial \varphi^{*}}{\partial t}\right)}{\gamma\left(\boldsymbol{V}_{\mathrm{C}}\right)} d V^{*}\right] d t
\end{gathered}
$$

Then we have:

$$
S\left[\left\{\varphi^{*}\left(x^{*}, t^{*}\right)\right\}, \boldsymbol{R}_{\mathbf{C}}(\boldsymbol{t})\right]=\int L^{\prime}\left[\left\{\varphi^{*}\right\},\left\{\frac{\partial \varphi^{*}}{\partial \boldsymbol{r}^{*}}\right\},\left\{\frac{\partial \varphi^{*}}{\partial t}\right\}, \boldsymbol{R}_{\mathbf{C}}, \boldsymbol{V}_{\mathbf{C}}\right] d t
$$

With

- $L^{\prime}\left[\left\{\varphi^{*}\right\},\left\{\frac{\partial \varphi^{*}}{\partial r^{*}}\right\},\left\{\frac{\partial \varphi^{*}}{\partial t}\right\}, \boldsymbol{R}_{\mathbf{C}}, \boldsymbol{V}_{\mathbf{C}}\right] \equiv \iiint \Lambda^{\prime *}\left(\varphi^{*}, \frac{\partial \varphi^{*}}{\partial r^{*}}, \frac{\partial \varphi^{*}}{\partial t}, \boldsymbol{R}_{\mathbf{C}}, \boldsymbol{V}_{\mathbf{C}}\right) d V^{*}$

- $\Lambda^{*}\left(\varphi^{*}, \frac{\partial \varphi^{*}}{\partial r^{*}}, \frac{\partial \varphi^{*}}{\partial t}, \boldsymbol{R}_{\mathrm{C}}, \boldsymbol{V}_{\mathrm{C}}\right) \equiv \frac{\Lambda^{*}\left(\varphi^{*}, \frac{\partial \varphi^{*}}{\partial r^{*}}, \gamma\left(\boldsymbol{V}_{\mathrm{C}}\right) \frac{\partial \varphi^{*}}{\partial t}\right)}{\gamma\left(\boldsymbol{V}_{\mathrm{C}}\right)}$

So we can calculate the 3-momentum as:

$$
\begin{gathered}
\boldsymbol{P}_{\boldsymbol{c}} \equiv \frac{\partial L^{\prime}}{\partial \boldsymbol{V}_{\mathbf{C}}}=\frac{\partial}{\partial \boldsymbol{V}_{\mathbf{C}}}\left[\frac{1}{\gamma\left(\boldsymbol{V}_{\mathbf{C}}\right)} \iiint \Lambda^{*}\left(\varphi^{*}, \frac{\partial \varphi^{*}}{\partial \boldsymbol{r}^{*}}, \gamma\left(\boldsymbol{V}_{\mathbf{C}}\right) \frac{\partial \varphi^{*}}{\partial t}\right) d V^{*}\right] \\
=\iiint \Lambda^{*}\left(\varphi^{*}, \frac{\partial \varphi^{*}}{\partial \boldsymbol{r}^{*}}, \gamma\left(\boldsymbol{V}_{\mathrm{C}}\right) \frac{\partial \varphi^{*}}{\partial t}\right) d V^{*} \frac{\partial}{\partial \boldsymbol{V}_{\mathbf{C}}} \frac{1}{\gamma\left(\boldsymbol{V}_{\mathrm{C}}\right)}+\frac{1}{\gamma\left(\boldsymbol{V}_{\mathbf{C}}\right)} \iiint \frac{\partial}{\partial \boldsymbol{V}_{\mathbf{C}}} \Lambda^{*}\left(\varphi^{*}, \frac{\partial \varphi^{*}}{\partial \boldsymbol{r}^{*}}, \gamma\left(\boldsymbol{V}_{\mathrm{C}}\right) \frac{\partial \varphi^{*}}{\partial t}\right) d V^{*}
\end{gathered}
$$




$$
\begin{aligned}
=\iiint \Lambda^{*}\left(\varphi^{*}, \frac{\partial \varphi^{*}}{\partial \boldsymbol{r}^{*}}, \gamma\left(\boldsymbol{V}_{\mathbf{C}}\right) \frac{\partial \varphi^{*}}{\partial t}\right) d V^{*}\left(-\gamma\left(\boldsymbol{V}_{\mathbf{C}}\right) \frac{\boldsymbol{V}_{\mathbf{C}}}{\boldsymbol{c}^{2}}\right) \\
+\frac{1}{\gamma\left(\boldsymbol{V}_{\mathbf{C}}\right)} \iiint \frac{\partial\left(\gamma\left(\boldsymbol{V}_{\mathbf{C}}\right) \frac{\partial \varphi^{*}}{\partial t}\right)}{\partial \boldsymbol{V}_{\mathbf{C}}} \frac{\partial}{\partial\left(\gamma\left(\boldsymbol{V}_{\mathbf{C}}\right) \frac{\partial \varphi^{*}}{\partial t}\right)} \Lambda^{*}\left(\varphi^{*}, \frac{\partial \varphi^{*}}{\partial \boldsymbol{r}^{*}}, \gamma\left(\boldsymbol{V}_{\mathbf{C}}\right) \frac{\partial \varphi^{*}}{\partial t}\right) d V^{*}
\end{aligned}
$$

$$
\begin{aligned}
& \text { But } \frac{\partial}{\partial V_{\mathrm{c}}} \frac{1}{\gamma\left(v_{\mathrm{C}}\right)}=-\gamma\left(\boldsymbol{V}_{\mathrm{C}}\right) \frac{V_{\mathrm{C}}}{c^{2}} ; \gamma\left(\boldsymbol{V}_{\mathrm{C}}\right) \frac{\partial \varphi^{*}}{\partial t}=\frac{\partial \varphi^{*}}{\partial t^{*}} \\
& \text { And } \frac{\partial\left(\gamma\left(V_{\mathrm{C}}\right) \frac{\partial \varphi^{*}}{\partial t}\right)}{\partial V_{\mathrm{C}}}=\frac{\partial \varphi^{*}}{\partial t} \frac{\partial \gamma\left(V_{\mathrm{C}}\right)}{\partial V_{\mathrm{C}}}=\frac{\partial \varphi^{*}}{\partial t} \frac{\partial \frac{1}{\sqrt{1-\frac{V_{\mathrm{C}}^{2}}{c^{2}}}}}{\partial V_{\mathrm{C}}}=\frac{\partial \varphi^{*}}{\partial t} \frac{-1}{2}\left(-2 \frac{V_{\mathrm{C}}}{c^{2}}\right) \frac{1}{\left(1-\frac{\gamma\left(v_{\mathrm{C}}\right)^{2}\left(\frac{d r_{\mathrm{a}}^{*}}{c^{2}}\right)^{2}}{c^{2}}\right)^{3 / 2}}=\frac{\partial \varphi^{*}}{\partial t} \frac{V_{\mathrm{C}}}{c^{2}} \gamma\left(V_{\mathrm{C}}\right)^{3} \\
& \boldsymbol{P}_{\boldsymbol{c}}=\iiint \Lambda^{*}\left(\varphi^{*}, \frac{\partial \varphi^{*}}{\partial \boldsymbol{r}^{*}}, \gamma\left(\boldsymbol{V}_{\mathbf{C}}\right) \frac{\partial \varphi^{*}}{\partial t}\right) d V^{*}\left(-\gamma\left(\boldsymbol{V}_{\mathbf{C}}\right) \frac{\boldsymbol{V}_{\mathbf{C}}}{\boldsymbol{c}^{2}}\right) \\
& +\frac{1}{\gamma\left(V_{\mathrm{C}}\right)} \iiint\left(\frac{\partial \varphi^{*}}{\partial t} \frac{V_{\mathrm{C}}}{c^{2}} \gamma\left(\boldsymbol{V}_{\mathrm{C}}\right)^{3}\right) \frac{\partial}{\partial\left(\frac{\partial \varphi^{*}}{\partial t^{*}}\right)} \Lambda^{*}\left(\varphi^{*}, \frac{\partial \varphi^{*}}{\partial \boldsymbol{r}^{*}}, \frac{\partial \varphi^{*}}{\partial t^{*}}\right) d V^{*} \\
& =\frac{\boldsymbol{V}_{\mathbf{C}}}{\boldsymbol{c}^{2}} \gamma\left(\boldsymbol{V}_{\mathbf{C}}\right) \iiint \Lambda^{*}\left(\varphi^{*}, \frac{\partial \varphi^{*}}{\partial \boldsymbol{r}^{*}}, \gamma\left(\boldsymbol{V}_{\mathrm{C}}\right) \frac{\partial \varphi^{*}}{\partial t}\right) d V^{*}(-1) \\
& +\iiint\left(\frac{\partial \varphi^{*}}{\partial t} \gamma\left(V_{\mathrm{C}}\right)\right) \frac{\partial}{\partial\left(\frac{\partial \varphi^{*}}{\partial t^{*}}\right)} \Lambda^{*}\left(\varphi^{*}, \frac{\partial \varphi^{*}}{\partial \boldsymbol{r}^{*}}, \frac{\partial \varphi^{*}}{\partial t^{*}}\right) d V^{*} \\
& =\frac{\boldsymbol{V}_{\mathbf{C}}}{\boldsymbol{c}^{2}} \gamma\left(\boldsymbol{V}_{\mathrm{C}}\right)\left[\iiint \Lambda^{*}\left(\varphi^{*}, \frac{\partial \varphi^{*}}{\partial \boldsymbol{r}^{*}}, \gamma\left(\boldsymbol{V}_{\mathbf{C}}\right) \frac{\partial \varphi^{*}}{\partial t}\right) d V^{*}(-1)+\iiint \frac{\partial \varphi^{*}}{\partial t^{*}} \frac{\partial}{\partial\left(\frac{\partial \varphi^{*}}{\partial t^{*}}\right)} \Lambda^{*}\left(\varphi^{*}, \frac{\partial \varphi^{*}}{\partial \boldsymbol{r}^{*}}, \frac{\partial \varphi^{*}}{\partial t^{*}}\right) d V^{*}\right] \\
& =\frac{\boldsymbol{V}_{\mathbf{C}}}{\boldsymbol{c}^{2}} \gamma\left(\boldsymbol{V}_{\mathrm{C}}\right) \iiint\left[\frac{\partial \varphi^{*}}{\partial t^{*}} \frac{\partial}{\partial\left(\frac{\partial \varphi^{*}}{\partial t^{*}}\right)} \Lambda^{*}-\Lambda^{*}\right] d V^{*}
\end{aligned}
$$

So we have again:

$$
\boldsymbol{P}_{\boldsymbol{c}}=\gamma \frac{E^{*}}{c^{2}} \boldsymbol{V}_{\mathbf{C}}
$$

where $E^{*} \equiv \iiint\left(\frac{\partial \varphi^{*}}{\partial t^{*}} \frac{\partial}{\partial\left(\frac{\partial \varphi^{*}}{\partial t^{*}}\right)} \Lambda^{*}-\Lambda^{*}\right) d V^{*}$ is the internal energy (associated to the hyperplane $t^{*}=$ ste)

And also: 


$$
M=M\left(t^{*}\right)=M\left(\int_{0}^{t} \frac{d t^{\prime}}{\gamma\left(t^{\prime}\right)}\right)=\frac{\iiint\left(\frac{\partial \varphi^{*}}{\partial t^{*}} \frac{\partial}{\partial\left(\frac{\partial \varphi^{*}}{\partial t^{*}}\right)} \Lambda^{*}-\Lambda^{*}\right) d V^{*}}{c^{2}}
$$

We see that we don't need to talk about closed system hypothesis or to have a 4 vector to demonstrate it (we don't even use the expression of any density Lagrangian).

We have to note, in the proof, the importance to freeze the right variable $\frac{\partial \varphi^{*}}{\partial t}$ (and not $\frac{\partial \varphi^{*}}{\partial t^{*}}$ ) in order to have the good expression.

\subsection{Momentum and energy for a field}

\subsubsection{Momentum}

We can also notice that $\boldsymbol{p}_{\varphi^{*}} \equiv \frac{\partial \varphi^{*}}{\partial \boldsymbol{r}^{*}} \frac{\partial \Lambda^{*}}{\partial \frac{\partial \varphi^{*}}{\partial t}}=\frac{\partial \varphi^{*}}{\partial \boldsymbol{r}^{*}} \frac{\partial \Lambda^{*}}{\partial\left(\frac{\partial \varphi^{*}}{\partial t^{*}}\right)}$, so $\boldsymbol{p}_{\varphi^{*}}=\frac{\partial \varphi^{*}}{\partial \boldsymbol{r}^{*}} \frac{\partial \Lambda^{*}}{\partial\left(\frac{\partial \varphi^{*}}{\partial t^{*}}\right)}$ as for material syste this is the same as the one we would have in the frame of the centre of mass $\mathrm{K}^{*}$.

More over the total momentum $\boldsymbol{P}_{\text {total }}$ associated to the Lagrangian $L^{\prime}\left[\left\{\varphi^{*}\right\},\left\{\frac{\partial \varphi^{*}}{\partial \boldsymbol{r}^{*}}\right\},\left\{\frac{\partial \varphi^{*}}{\partial t}\right\}, \boldsymbol{R}_{\mathrm{C}}, \boldsymbol{V}_{\mathrm{C}}\right]$ is $\boldsymbol{P}_{\text {total }}=\iiint \frac{\partial \varphi^{*}}{\partial \boldsymbol{r}^{*}} \frac{\partial \Lambda^{*}}{\partial \frac{\partial \varphi^{*}}{\partial t}} d V^{*}+\frac{\partial L^{\prime}}{\partial v_{\mathrm{c}}}=\iiint \boldsymbol{p}_{\varphi^{*}} d V^{*}+\boldsymbol{P}_{\boldsymbol{c}}=\boldsymbol{P}_{\boldsymbol{c}}$ since by definition of $\mathrm{K}^{*}: \iiint \boldsymbol{p}_{\varphi^{*}} d V^{*} \equiv 0$. As the material system above, we obtain as it should the total momentum is the one associated to the mass center.

Proof:

$\boldsymbol{p}_{\varphi^{*}} \equiv \frac{\partial \varphi^{*}}{\partial \boldsymbol{r}^{*}} \frac{\partial \Lambda^{*}}{\partial \frac{\partial \varphi^{*}}{\partial t}}=\frac{\partial \varphi^{*}}{\partial \boldsymbol{r}^{*}} \frac{\partial \frac{\Lambda^{*}\left(\varphi^{*}, \frac{\partial \varphi^{*}}{\partial \boldsymbol{r}^{*}}, \gamma \frac{\partial \varphi^{*}}{\partial t}, \boldsymbol{R}_{\mathbf{C}}, V_{\mathrm{C}}\right)}{\gamma}}{\partial \frac{\partial \varphi^{*}}{\partial t}}=\frac{\partial \varphi^{*}}{\partial \boldsymbol{r}^{*}}\left(\frac{\partial\left(\gamma \frac{\partial \varphi^{*}}{\partial t}\right)}{\partial \frac{\partial \varphi^{*}}{\partial t}}\right)\left(\frac{\partial \frac{\Lambda^{*}\left(\varphi^{*}, \frac{\partial \varphi^{*}}{\partial \boldsymbol{r}^{*}}, \gamma \frac{\partial \varphi^{*}}{\partial t}, \boldsymbol{R}_{\mathbf{C}}, V_{\mathrm{C}}\right)}{\gamma}}{\partial\left(\gamma \frac{\partial \varphi^{*}}{\partial t}\right)}\right)=$

$\frac{\partial \varphi^{*}}{\partial r^{*}}(\gamma)\left(\frac{1}{\gamma} \frac{\partial \Lambda^{*}\left(\varphi^{*}, \frac{\partial \varphi^{*}}{\partial r^{*}}, \frac{\partial \varphi^{*}}{\partial t}, \boldsymbol{R}_{\mathrm{C}}, \boldsymbol{V}_{\mathrm{C}}\right)}{\partial\left(\frac{\partial \varphi^{*}}{\partial t^{*}}\right)}\right)=\frac{\partial \varphi^{*}}{\partial \boldsymbol{r}^{*}} \frac{\partial \Lambda^{*}\left(\varphi^{*}, \frac{\partial \varphi^{*}}{\partial r^{*},}, \frac{\partial \varphi^{*}}{\partial t}, \boldsymbol{R}_{\mathrm{C}}, V_{\mathrm{C}}\right)}{\partial\left(\frac{\partial \varphi^{*}}{\partial t^{*}}\right)}=\frac{\partial \varphi^{*}}{\partial r^{*}} \frac{\partial \Lambda^{*}}{\partial\left(\frac{\partial \varphi^{*}}{\partial t^{*}}\right)}$, so $\boldsymbol{p}_{\varphi^{*}}=\frac{\partial \varphi^{*}}{\partial \boldsymbol{r}^{*}} \frac{\partial L^{*}}{\partial \frac{d r_{a}^{*}}{d t^{*}}}$

\subsubsection{Energy}

By definition the energy is: $E^{\prime} \equiv \iiint \frac{\partial \Lambda^{*}}{\partial \frac{\partial \varphi^{*}}{\partial t} \varphi^{*}} \partial t \quad d V^{*}+\frac{\partial L^{\prime}}{\partial V_{\mathbf{c}}} \boldsymbol{V}_{\boldsymbol{c}}-L^{\prime}$

We can re-express it as:

$$
\begin{aligned}
& E^{\prime}=\iiint \frac{\partial \Lambda^{*}}{\partial \frac{\partial \varphi^{*}}{\partial t}} \frac{\partial \varphi^{*}}{\partial t} d V^{*}+\boldsymbol{P}_{\boldsymbol{c}} \boldsymbol{V}_{\boldsymbol{c}}-\frac{L^{* \prime}}{\gamma} \text { since } L^{\prime}=\frac{L^{* \prime}}{\gamma} \\
& =\iiint \frac{\partial \Lambda^{*}}{\partial\left(\frac{\partial \varphi^{*}}{\partial t^{*}}\right)} \frac{\partial \varphi^{*}}{\partial t} d V^{*}+\left(\gamma \frac{E^{*}}{c^{2}} \cdot \boldsymbol{V}_{\boldsymbol{c}}\right) \boldsymbol{V}_{\boldsymbol{c}}-\frac{L^{* \prime}}{\gamma} \text { since } \boldsymbol{p}_{\varphi^{*}} \equiv \frac{\partial \Lambda^{*}}{\partial \frac{\partial \varphi^{*}}{\partial t}}=\frac{\partial \Lambda^{*}}{\partial\left(\frac{\partial \varphi^{*}}{\partial t^{*}}\right)}
\end{aligned}
$$




$$
\begin{aligned}
& =\iiint \frac{\partial \Lambda^{*}}{\partial\left(\frac{\partial \varphi^{*}}{\partial t^{*}}\right)} \frac{\partial \varphi^{*}}{\partial t} d V^{*}-\frac{L^{* \prime}}{\gamma}+\gamma \frac{E^{*}}{c^{2}} \cdot \boldsymbol{V}_{\boldsymbol{c}}{ }^{2} \\
& =\iiint \frac{\partial \Lambda^{*}}{\partial\left(\frac{\partial \varphi^{*}}{\partial t^{*}}\right)} \frac{\partial \varphi^{*}}{\gamma \partial t^{*}} d V^{*}-\frac{L^{* \prime}}{\gamma}+\gamma \frac{E^{*}}{c^{2}} \cdot \boldsymbol{V}_{\boldsymbol{c}}{ }^{2}=\left(\iiint \frac{\partial \Lambda^{*}}{\partial\left(\frac{\partial \varphi^{*}}{\partial t^{*}}\right)} \frac{\partial \varphi^{*}}{\partial t^{*}} d V^{*}-L^{* \prime}\right) \frac{1}{\gamma}+\gamma \frac{E^{*}}{c^{2}} \cdot \boldsymbol{V}_{\boldsymbol{c}}{ }^{2} \\
& =\frac{E^{*}}{\gamma}+\gamma \frac{E^{*}}{c^{2}} \cdot \boldsymbol{V}_{\boldsymbol{c}}{ }^{2}=\frac{E^{*}}{\gamma}+\gamma \frac{E^{*}}{c^{2}} \cdot \boldsymbol{V}_{\boldsymbol{c}}{ }^{2}=\frac{E^{*}+\gamma^{2} \frac{E^{*}}{c^{2}} \cdot \boldsymbol{V}_{\boldsymbol{c}}{ }^{2}}{\gamma}=E^{*} \frac{1+\gamma^{2} \cdot \beta^{2}}{\gamma}=E^{*} \frac{1+\frac{\beta^{2}}{1-\beta^{2}}}{\gamma} \\
& =\frac{1-\beta^{2}+\beta^{2}}{1-\beta^{2}} \\
& =E^{*} \frac{1}{\gamma}=E^{*} \frac{1-\beta^{2}}{\gamma}=E^{*} \frac{\gamma^{2}}{\gamma}=\gamma E^{*}
\end{aligned}
$$

So we have, as it should:

$$
E^{\prime}=E=\gamma E^{*}
$$

We can also conventionally note: $E=E^{*}+(\gamma-1) E^{*}$ where we observe, for a closed system ( $\mathrm{E}=\mathrm{cte})$, an exchange of Energy between the internal energy $E^{*}$ and the kinetic energy $(\gamma-1) E^{*}$, the one depending of the center of mass.

\subsection{The Euler-Lagrange equation for the internal field and the mass center}

The Euler-Lagrange equations in $\mathrm{K}$ are :

$$
\begin{aligned}
& \frac{d}{d t} \frac{\partial}{\partial \boldsymbol{V}_{\mathbf{C}}} L^{\prime}\left[\left\{\varphi^{*}\right\},\left\{\frac{\partial \varphi^{*}}{\partial \boldsymbol{r}^{*}}\right\}\right.\left.,\left\{\frac{\partial \varphi^{*}}{\partial t}\right\}, \boldsymbol{R}_{\mathrm{C}}, \boldsymbol{V}_{\mathbf{C}}\right]=\frac{\partial}{\partial \boldsymbol{R}_{\mathbf{C}}} L^{\prime}\left[\left\{\varphi^{*}\right\},\left\{\frac{\partial \varphi^{*}}{\partial \boldsymbol{r}^{*}}\right\},\left\{\frac{\partial \varphi^{*}}{\partial t}\right\}, \boldsymbol{R}_{\mathbf{C}}, \boldsymbol{V}_{\mathrm{C}}\right] \\
&<=>\frac{d}{d t}\left(\gamma\left(\boldsymbol{V}_{\mathbf{C}}\right) \frac{E^{*}}{c^{2}} \boldsymbol{V}_{\mathbf{C}}\right)=\frac{\partial}{\partial \boldsymbol{R}_{\mathbf{C}}} L^{\prime}\left[\left\{\varphi^{*}\right\},\left\{\frac{\partial \varphi^{*}}{\partial \boldsymbol{r}^{*}}\right\},\left\{\frac{\partial \varphi^{*}}{\partial t}\right\}, \boldsymbol{R}_{\mathrm{C}}, \boldsymbol{V}_{\mathbf{C}}\right]
\end{aligned}
$$

And we find in $\mathrm{K}^{*}$ :

$\frac{\partial}{\partial t}\left(\frac{\partial \Lambda^{\prime *}}{\partial \frac{\partial \varphi^{*}}{\partial t}}\right)+\frac{\partial}{\partial r^{*}}\left(\frac{\partial \Lambda^{\prime *}}{\partial \frac{\partial \varphi^{*}}{\partial r^{*}}}\right)=\frac{\partial \Lambda^{\prime *}}{\partial \varphi^{*}}=\frac{1}{\gamma\left(\boldsymbol{V}_{\mathbf{C}}\right)} \frac{\partial \Lambda^{*}}{\partial \varphi^{*}}$

With $\Lambda^{*}\left(\varphi^{*}, \frac{\partial \varphi^{*}}{\partial \boldsymbol{r}^{*}}, \frac{\partial \varphi^{*}}{\partial t}, \boldsymbol{R}_{\mathbf{C}}, \boldsymbol{V}_{\mathbf{C}}\right) \equiv \frac{\Lambda^{*}\left(\varphi^{*}, \frac{\partial \varphi^{*}}{\partial r^{*}}, \gamma\left(\boldsymbol{V}_{\mathbf{C}}\right) \frac{\partial \varphi^{*}}{\partial t}\right)}{\gamma\left(\boldsymbol{V}_{\mathbf{C}}\right)}$

That we can show is equivalent of the 4-dimensional equation in $\mathrm{K}^{*}$ :

$<=>\frac{\partial}{\partial x^{i}}\left(\frac{\partial \Lambda^{*}}{\partial \frac{\partial \varphi^{*}}{\partial x^{*}}}\right)=\frac{\partial \Lambda^{*}}{\partial \varphi^{*}}$

As above for the material system, we obtain the same equation that we should obtain for the dynamic in a $\mathrm{K}^{*}$ frame: the local Galilean frame. 


\section{Proof:}

We start from the general Lagrangian

$S[\{\varphi(x, t)\}]=\int\left[\iiint \Lambda^{*}\left(\varphi^{*}, \frac{\partial \varphi^{*}}{\partial \boldsymbol{r}^{*}}, \gamma \frac{\partial \varphi^{*}}{\partial t}\right) d V^{*}\right] \frac{d t}{\gamma}=\int\left[\iiint \Lambda^{* *}\left(\varphi^{*}, \frac{\partial \varphi^{*}}{\partial \boldsymbol{r}^{*}}, \frac{\partial \varphi^{*}}{\partial t}, \boldsymbol{R}_{\mathbf{C}}, \boldsymbol{V}_{\mathbf{C}}\right) d V^{*}\right] d t$

With $\Lambda^{\prime *}\left(\varphi^{*}, \frac{\partial \varphi^{*}}{\partial \boldsymbol{r}^{*}}, \frac{\partial \varphi^{*}}{\partial t}, \boldsymbol{R}_{\mathbf{C}}, \boldsymbol{V}_{\mathbf{C}}\right) \equiv \frac{\Lambda^{*}\left(\varphi^{*}, \frac{\partial \varphi^{*}}{\partial r^{*}}, \gamma \frac{\partial \varphi^{*}}{\partial t}\right)}{\gamma}$

The variation of the action gives:

$\delta S[\{\varphi(x, t)\}]=\int\left[\iint \frac{\partial \Lambda^{\prime *}}{\partial \varphi^{*}} \delta \varphi^{*}+\frac{\partial \Lambda^{\prime *}}{\partial \frac{\partial \varphi^{*}}{\partial \boldsymbol{r}^{*}}} \delta \frac{\partial \varphi^{*}}{\partial \boldsymbol{r}^{*}}+\frac{\partial \Lambda^{\prime *}}{\partial \frac{\partial \varphi^{*}}{\partial t}} \delta \frac{\partial \varphi^{*}}{\partial t}\right] d V^{*} d t$

$=\int\left[\iiint \frac{\partial \Lambda^{\prime *}}{\partial \varphi^{*}} \delta \varphi^{*}+\left\{\frac{\partial}{\partial \boldsymbol{r}^{*}}\left(\frac{\partial \Lambda^{\prime *}}{\partial \frac{\partial \varphi^{*}}{\partial \boldsymbol{r}^{*}}} \delta \varphi^{*}\right)-\frac{\partial}{\partial \boldsymbol{r}^{*}}\left(\frac{\partial \Lambda^{\prime *}}{\partial \frac{\partial \varphi^{*}}{\partial \boldsymbol{r}^{*}}}\right) \delta \varphi^{*}\right\}+\left\{\frac{\partial}{\partial t}\left(\frac{\partial \Lambda^{\prime *}}{\partial \frac{\partial \varphi^{*}}{\partial \boldsymbol{r}^{*}}} \delta \varphi^{*}\right)-\frac{\partial}{\partial t}\left(\frac{\partial \Lambda^{\prime *}}{\partial \frac{\partial \varphi^{*}}{\partial t}}\right) \delta \varphi^{*}\right\}\right] d V^{*} d t$

$=\int\left[\iint\left\{\frac{\partial}{\partial \boldsymbol{r}^{*}}\left(\frac{\partial \Lambda^{\prime *}}{\partial \frac{\partial \varphi^{*}}{\partial \boldsymbol{r}^{*}}} \delta \varphi^{*}\right)+\frac{\partial}{\partial t}\left(\frac{\partial \Lambda^{\prime *}}{\partial \frac{\partial \varphi^{*}}{\partial \boldsymbol{r}^{*}}} \delta \varphi^{*}\right)\right\}+\delta \varphi^{*}\left\{\frac{\partial \Lambda^{\prime *}}{\partial \varphi^{*}}-\frac{\partial}{\partial \boldsymbol{r}^{*}}\left(\frac{\partial \Lambda^{\prime *}}{\partial \frac{\partial \varphi^{*}}{\partial \boldsymbol{r}^{*}}}\right)-\frac{\partial}{\partial t}\left(\frac{\partial \Lambda^{\prime *}}{\partial \frac{\partial \varphi^{*}}{\partial t}}\right)\right\}\right] d V^{*} d t$

The least action principle tells us that

$$
\delta S[\{\varphi(x, t)\}]=0
$$

$$
\begin{aligned}
& =>\frac{\partial}{\partial t}\left(\frac{\partial \Lambda^{\prime *}}{\partial \frac{\partial \varphi^{*}}{\partial t}}\right)+\frac{\partial}{\partial \boldsymbol{r}^{*}}\left(\frac{\partial \Lambda^{\prime *}}{\partial \frac{\partial \varphi^{*}}{\partial \boldsymbol{r}^{*}}}\right)=\frac{\partial \Lambda^{\prime *}}{\partial \varphi^{*}}=\frac{1}{\gamma} \frac{\partial \Lambda^{*}}{\partial \varphi^{*}} \\
& <=>\gamma \frac{\partial}{\partial t}\left(\frac{\partial \Lambda^{\prime *}}{\partial \frac{\partial \varphi^{*}}{\partial t}}\right)+\gamma \frac{\partial}{\partial \boldsymbol{r}^{*}}\left(\frac{\partial \Lambda^{\prime *}}{\partial \frac{\partial \varphi^{*}}{\partial \boldsymbol{r}^{*}}}\right)=\frac{\partial \Lambda^{*}}{\partial \varphi^{*}} \\
& <=>\gamma \frac{\partial}{\partial t}\left(\frac{\partial \Lambda^{*}}{\partial\left(\frac{\partial \varphi^{*}}{\partial t^{*}}\right)}\right)+\gamma \frac{\partial}{\partial r^{*}}\left(\frac{\partial \Lambda^{\prime *}}{\partial \frac{\partial \varphi^{*}}{\partial r^{*}}}\right)=\frac{\partial \Lambda^{*}}{\partial \varphi^{*}} \text { since } \frac{\partial \Lambda^{*}}{\partial \frac{\partial \varphi^{*}}{\partial t}}=\frac{\partial \Lambda^{*}}{\partial\left(\frac{\partial \varphi^{*}}{\partial t^{*}}\right)}
\end{aligned}
$$

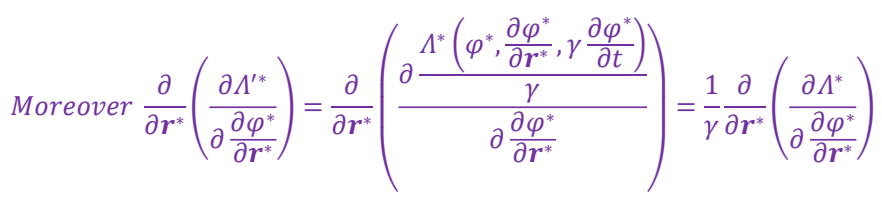

$$
\begin{aligned}
& \text { And } \gamma \frac{\partial}{\partial t}\left(\frac{\partial \Lambda^{*}}{\partial\left(\frac{\partial \varphi^{*}}{\partial t^{*}}\right)}\right)=\frac{\partial}{\partial t^{*}}\left(\frac{\partial \Lambda^{*}}{\partial\left(\frac{\partial \varphi^{*}}{\partial t^{*}}\right)}\right) \\
& \frac{\partial}{\partial t^{*}}\left(\frac{\partial \Lambda^{*}}{\partial\left(\frac{\partial \varphi^{*}}{\partial t^{*}}\right)}\right)+\frac{\partial}{\partial \boldsymbol{r}^{*}}\left(\frac{\partial \Lambda^{*}}{\partial \frac{\partial \varphi^{*}}{\partial \boldsymbol{r}^{*}}}\right)=\frac{\partial \Lambda^{*}}{\partial \varphi^{*}} \\
& <=>\frac{\partial}{\partial \boldsymbol{x}^{i^{*}}}\left(\frac{\partial \Lambda^{*}}{\partial \frac{\partial \varphi^{*}}{\partial \boldsymbol{x}^{*}}}\right)=\frac{\partial \Lambda^{*}}{\partial \varphi^{*}}
\end{aligned}
$$




\section{Example: Application to the Einsteinian gravitational field}

According to [1]:

$$
S\left[\left\{g_{i k}(x, t)\right\}\right]=\frac{-c^{3}}{16 \pi k} \iiint \int(R-2 \Lambda) \sqrt{-g} d \Omega
$$

Where $\mathrm{R}$ is the Ricci scalar.

$$
\delta S\left[\left\{g_{i k}(x, t)\right\}\right]=\frac{-c^{3}}{16 \pi k} \delta \iiint \int(R-2 \Lambda) \sqrt{-g} d \Omega=\frac{-c^{3}}{16 \pi k} \delta \iiint \int(G-2 \Lambda) \sqrt{-g} d \Omega
$$

With :

○ $G\left(g_{i k}, \frac{\partial g_{i k}}{\partial r}, \frac{\partial g_{i k}}{\partial t}\right)=g^{i k}\left(\Gamma_{i l}^{m} \Gamma_{k m}^{l}-\Gamma_{i k}^{l} \Gamma_{l m}^{m}\right)$

○ $\Lambda$ the Einstein-(Lemaître) cosmological constant

For linear transformation $\Gamma_{i l}^{m}$ behave like tensor, so $\mathrm{G}$ behaves as a scalar.

We consider a context where the space is Lorentzian to infinity. The (linear) Lorentz transformation means, in this case, a modification of the speed for the part of the of the observers (associated to the current frame) to infinity. The modification of the coordinate system for other observers is meanwhile not directly evident but allowed.

$$
\begin{gathered}
S\left[\left\{g_{i k}(x, t)\right\}\right]=\frac{-c^{3}}{16 \pi k} \iiint \int\left(G\left(g_{i k}, \frac{\partial g_{i k}}{\partial \boldsymbol{r}}, \frac{\partial g_{i k}}{\partial t}\right)-2 \Lambda\right) \sqrt{-g} d \Omega . \\
=\frac{-c^{3}}{16 \pi k} \iiint \int\left(G^{*}\left(g_{i k}{ }^{*}, \frac{\partial g_{i k}{ }^{*}}{\partial \boldsymbol{r}^{*}}, \frac{\partial g_{i k}{ }^{*}}{\partial t^{*}}\right)-2 \Lambda\right) \sqrt{-g^{*}} d \Omega^{*} \\
=\frac{-c^{4}}{16 \pi k} \int\left[\iiint\left(G^{*}\left(g_{i k}{ }^{*}, \frac{\partial g_{i k}{ }^{*}}{\partial \boldsymbol{r}^{*}}, \frac{\partial g_{i k}}{\partial t^{*}}\right)-2 \Lambda\right) \sqrt{-g^{*}} d V^{*}\right] d t^{*} \\
=\frac{-c^{4}}{16 \pi k} \int\left[\iiint\left(G^{*}\left(g_{i k}{ }^{*}, \frac{\partial g_{i k}{ }^{*}}{\partial \boldsymbol{r}^{*}}, \gamma\left(\boldsymbol{V}_{\mathbf{C}}\right) \frac{\partial g_{i k}{ }^{*}}{\partial t}\right)-2 \Lambda\right) \sqrt{-g^{*}} d V^{*}\right] \frac{d t}{\gamma\left(\boldsymbol{V}_{\mathbf{C}}\right)} \\
=\frac{-c^{4}}{16 \pi k} \int\left[\iiint \frac{\left(G^{*}\left(g_{i k}{ }^{*}, \frac{\partial g_{i k}}{\partial \boldsymbol{r}^{*}}, \gamma\left(\boldsymbol{V}_{\mathbf{C}}\right) \frac{\partial g_{i k}{ }^{*}}{\partial t}\right)-2 \Lambda\right) \sqrt{-g^{*}}}{\gamma\left(\boldsymbol{V}_{\mathbf{C}}\right)} d V^{*}\right] d t \\
=\frac{-c^{4}}{16 \pi k} \int\left[\iiint\left(\left(G^{*}-2 \Lambda^{\prime}\right) \sqrt{-g^{\prime *}}\right)\left(g_{i k}{ }^{*}, \frac{\partial g_{i k}^{*}}{\partial \boldsymbol{r}^{*}}, \frac{\partial g_{i k}{ }^{*}}{\partial t}, \boldsymbol{R}_{\mathbf{C}}, \boldsymbol{V}_{\mathbf{C}}\right) d V^{*}\right] d t
\end{gathered}
$$

Then we have 


$$
S\left[\left\{g_{i k}{ }^{*}\left(x^{*}, t^{*}\right)\right\}, \boldsymbol{R}_{\mathbf{C}}(\boldsymbol{t})\right]=\int L^{\prime}\left[\left\{g_{i k}^{*}\right\},\left\{\frac{\partial g_{i k}^{*}}{\partial \boldsymbol{r}^{*}}\right\},\left\{\frac{\partial g_{i k}^{*}}{\partial t}\right\}, \boldsymbol{R}_{\mathbf{C}}, \boldsymbol{V}_{\mathbf{C}}\right] d t
$$

With

- $L^{\prime}\left[\left\{g_{i k}^{*}\right\},\left\{\frac{\partial g_{i k}^{*}}{\partial r^{*}}\right\},\left\{\frac{\partial g_{i k}^{*}}{\partial t}\right\}, \boldsymbol{R}_{\mathbf{C}}, \boldsymbol{V}_{\mathbf{C}}\right] \equiv \frac{-c^{4}}{16 \pi k} \iiint\left(\left(G^{*}-2 \Lambda^{\prime}\right) \sqrt{-g^{\prime *}}\right)\left(g_{i k}{ }^{*}, \frac{\partial g_{i k}}{\partial \boldsymbol{r}^{*}}, \frac{\partial g_{i k}{ }^{*}}{\partial t}, \boldsymbol{R}_{\mathbf{C}}, \boldsymbol{V}_{\mathbf{C}}\right) d V^{*}$

- $\left(\left(G^{\prime *}-2 \Lambda^{\prime}\right) \sqrt{-g^{\prime *}}\right)\left(g_{i k}{ }^{*}, \frac{\partial g_{i k}{ }^{*}}{\partial \boldsymbol{r}^{*}}, \frac{\partial g_{i k^{*}}}{\partial t}, \boldsymbol{R}_{\mathbf{C}}, \boldsymbol{V}_{\mathbf{C}}\right) \equiv \frac{\left(\left(G^{*}-2 \Lambda\right) \sqrt{-g^{*}}\right)\left(g_{i k^{*}}, \frac{\partial g_{i k^{*}}}{\partial \boldsymbol{r}^{*}}, \gamma\left(\boldsymbol{V}_{\mathbf{C}}\right) \frac{\partial g_{i k}}{\partial t}\right)}{\gamma\left(\boldsymbol{V}_{\mathbf{C}}\right)}$

Repeating the same calculation for the scalar field we have (as explained in [1], the summation over the metric coefficients is really applied for the 10 coefficients which are independent):

$$
\boldsymbol{P}_{\boldsymbol{c}}=\gamma \frac{E^{*}}{c^{2}} \boldsymbol{V}_{\mathbf{C}}
$$

where $E^{*} \equiv \frac{-c^{4}}{16 \pi k} \iiint\left(\frac{\partial g_{i k}{ }^{*}}{\partial t^{*}} \frac{\partial\left(G^{*} \sqrt{-g^{*}}\right)}{\partial\left(\frac{\partial g_{i k}^{*}}{\partial t^{*}}\right)}-\left(G^{*}-2 \Lambda\right) \sqrt{-g^{*}}\right) d V^{*}$ is the internal energy

(associated to the hyperplane $t^{*}=c t e$ )

This is of course coherent with the 4-momentum of [1], paragraph 96.

We have therefore:

$$
M=M\left(t^{*}\right)=M\left(\int_{0}^{t} \frac{d t^{\prime}}{\gamma\left(t^{\prime}\right)}\right)=\frac{-c^{2}}{16 \pi k} \iiint\left(\frac{\partial g_{i k}}{\partial t^{*}} \frac{\partial\left(G^{*} \sqrt{-g^{*}}\right)}{\partial\left(\frac{\partial g_{i k}^{*}}{\partial t^{*}}\right)}-\left(G^{*}-2 \Lambda\right) \sqrt{-g^{*}}\right) d V^{*}
$$

The Euler-Lagrange equations:

- For the gravitational free particle of mass in $\mathrm{K}$ :

$\frac{d}{d t} \frac{\partial}{\partial \boldsymbol{V}_{\mathbf{C}}} L^{\prime}\left[\left\{g_{i k}^{*}\right\},\left\{\frac{\partial g_{i k}^{*}}{\partial \boldsymbol{r}^{*}}\right\},\left\{\frac{\partial g_{i k}^{*}}{\partial t}\right\}, \boldsymbol{R}_{\mathbf{C}}, \boldsymbol{V}_{\mathbf{C}}\right]=\frac{\partial}{\partial \boldsymbol{R}_{\mathbf{C}}} L^{\prime}\left[\left\{g_{i k}^{*}\right\},\left\{\frac{\partial g_{i k}^{*}}{\partial \boldsymbol{r}^{*}}\right\},\left\{\frac{\partial g_{i k}{ }^{*}}{\partial t}\right\}, \boldsymbol{R}_{\mathbf{C}}, \boldsymbol{V}_{\mathbf{C}}\right]$

$<=>\frac{d}{d t}\left(\gamma \frac{E^{*}}{c^{2}} \boldsymbol{V}_{\mathbf{C}}\right)=\frac{\partial}{\partial \boldsymbol{R}_{\mathbf{C}}} L^{\prime}\left[\left\{g_{i k}{ }^{*}\right\},\left\{\frac{\partial g_{i k}{ }^{*}}{\partial \boldsymbol{r}^{*}}\right\},\left\{\frac{\partial g_{i k}{ }^{*}}{\partial t}\right\}, \boldsymbol{R}_{\mathrm{C}}, \boldsymbol{V}_{\mathrm{C}}\right]$

- For the internal (Einsteinian) gravitational field in $\mathrm{K}^{*}([1])$ :

$\frac{\partial}{\partial t}\left(\frac{\partial\left(\left(G^{\prime *}-2 \Lambda^{\prime}\right) \sqrt{-g^{\prime *}}\right)}{\frac{\partial g_{i k}^{*}}{\partial t}}\right)+\frac{\partial}{\partial \boldsymbol{r}^{*}}\left(\frac{\partial\left(\left(G^{\prime *}-2 \Lambda^{\prime}\right) \sqrt{-g^{\prime *}}\right)}{\frac{\partial g_{i k}^{*}}{\partial \boldsymbol{r}^{*}}}\right)=\frac{\partial\left(\left(G^{\prime *}-2 \Lambda^{\prime}\right) \sqrt{-g^{\prime *}}\right)}{\partial g_{i k^{*}}}=\frac{1}{\gamma\left(\boldsymbol{V}_{\mathbf{C}}\right)} \frac{\partial\left(\left(G^{*}-2 \Lambda\right) \sqrt{-g^{*}}\right)}{\partial g_{i k^{*}}}$

With

$\left(\left(G^{*}-2 \Lambda^{\prime}\right) \sqrt{-g^{\prime *}}\right)\left(g_{i k}{ }^{*}, \frac{\partial g_{i k}{ }^{*}}{\partial \boldsymbol{r}^{*}}, \frac{\partial g_{i k^{*}}}{\partial t}, \boldsymbol{R}_{\mathbf{C}}, \boldsymbol{V}_{\mathbf{C}}\right) \equiv \frac{\left(\left(G^{*}-2 \Lambda\right) \sqrt{-g^{*}}\right)\left(g_{i k^{*}}, \frac{\partial g_{i k}{ }^{*}}{\partial \boldsymbol{r}^{*}}, \gamma\left(\boldsymbol{V}_{\mathbf{C}}\right) \frac{\partial g_{i k}}{\partial t}\right)}{\gamma\left(\boldsymbol{V}_{\mathbf{C}}\right)}$

Then we have equivalently 
$\frac{\partial}{\partial t}\left(\frac{\partial G^{\prime *} \sqrt{-g^{*}}}{\partial \frac{\partial g_{i k}^{*}}{\partial t}}\right)+\frac{\partial}{\partial \boldsymbol{r}^{*}}\left(\frac{\partial G^{\prime *} \sqrt{-g^{*}}}{\partial \frac{\partial g_{i k}^{*}}{\partial \boldsymbol{r}^{*}}}\right)=\frac{1}{\gamma\left(\boldsymbol{V}_{\mathbf{C}}\right)} \frac{\partial\left(G^{*}-2 \Lambda\right) \sqrt{-g^{*}}}{\partial g_{i k}{ }^{*}}$

$<=>\frac{\partial}{\partial x^{l^{*}}}\left(\frac{\partial G^{*} \sqrt{-g^{*}}}{\partial \frac{\partial g_{i k}^{*}}{\partial x^{l^{*}}}}\right)=\frac{\partial\left(G^{*}-2 \Lambda\right) \sqrt{-g^{*}}}{\partial g_{i k}{ }^{*}}$

$<=>R_{i k}{ }^{*}-\frac{1}{2} g_{i k}{ }^{*} \cdot R^{*}+g_{i k}{ }^{*} \cdot \Lambda=0<=>R_{i k}{ }^{*}=4 \Lambda$

○ And also $E^{\prime}=E=\gamma E^{*}$

Remark: We can observe the impact of the cosmological constant on the mass of every given volume $V^{*}$ studied which are increased/decreased (depending on the sign) by the value

$\Delta M_{\Lambda}=\frac{-c^{2}}{8 \pi k} \Lambda \iiint\left(\sqrt{-g^{*}}\right) d V^{*}$ (there is a divergence for an infinite space...). That is why we can say that the cosmological constant gives mass to the gravitation field (mentioned by Einstein in 1918 in a discussion with Schrödinger, cf. [26]). However it is not a kind of mass that is seen by gravitational waves, that is to say as a more conventional field theory. In the latter sense the gravitation has indeed no a mass ("the graviton has no mass").

Proof:

I only gives the proof for the equation of motion (But it is quasi totally the same as for a scalar field)

$$
\begin{array}{r}
S\left[\left\{g_{i k}(x, t)\right\}\right]=\int\left[\iiint \Lambda^{*}\left(g_{i k}{ }^{*}, \frac{\partial g_{i k}{ }^{*}}{\partial \boldsymbol{r}^{*}}, \gamma\left(\boldsymbol{V}_{\mathbf{C}}\right) \frac{\partial g_{i k}{ }^{*}}{\partial t}\right) d V^{*}\right] \frac{d t}{\gamma\left(\boldsymbol{V}_{\mathbf{C}}\right)} \\
=\int\left[\iiint \Lambda^{\prime *}\left(\varphi^{*}, \frac{\partial g_{i k}{ }^{*}}{\partial \boldsymbol{r}^{*}}, \frac{\partial g_{i k}{ }^{*}}{\partial t}, \boldsymbol{R}_{\mathbf{C}}, \boldsymbol{V}_{\mathbf{C}}\right) d V^{*}\right] d t
\end{array}
$$

With $\Lambda^{\prime *}\left(g_{i k}{ }^{*}, \frac{\partial g_{i k}{ }^{*}}{\partial \boldsymbol{r}^{*}}, \frac{\partial g_{i k}{ }^{*}}{\partial t}, \boldsymbol{R}_{\mathbf{C}}, \boldsymbol{V}_{\mathbf{C}}\right) \equiv \frac{\Lambda^{*}\left(g_{i k^{*}}, \frac{\partial g_{i k}{ }^{*}}{\partial r^{*}}, \gamma\left(\boldsymbol{V}_{\mathbf{C}}\right) \frac{\partial g_{i k}{ }^{*}}{\partial t}\right)}{\gamma\left(\boldsymbol{V}_{\mathbf{C}}\right)}=\frac{\left(G^{*}\left(g_{i k^{*}}, \frac{\partial g_{i k}{ }^{*}}{\partial \boldsymbol{r}^{*}} \gamma\left(\boldsymbol{V}_{\mathbf{C}}\right) \frac{\partial g_{i k}{ }^{*}}{\partial t}\right)-2 \Lambda\right) \sqrt{-g^{*}}}{\gamma\left(\boldsymbol{V}_{\mathbf{C}}\right)}$

The variation of the action ("in the field" of the center of mass) gives:

$$
\begin{aligned}
& \delta S\left[\left\{g_{i k}(x, t)\right\}\right]_{R_{\mathbf{C}}(t)}=\int\left[\iiint \frac{\partial \Lambda^{\prime *}}{\partial g_{i k}{ }^{*}} \delta g_{i k}{ }^{*}+\frac{\partial \Lambda^{\prime *}}{\partial \frac{\partial \varphi^{*}}{\partial \boldsymbol{r}^{*}}} \delta \frac{\partial g_{i k}{ }^{*}}{\partial \boldsymbol{r}^{*}}+\frac{\partial \Lambda^{\prime *}}{\partial \frac{\partial g_{i k}{ }^{*}}{\partial t}} \delta \frac{\partial g_{i k}{ }^{*}}{\partial t}\right] d V^{*} d t \\
& =\int\left[\iiint \frac{\partial \Lambda^{\prime *}}{\partial g_{i k^{*}}} \delta g_{i k^{*}}+\left\{\frac{\partial}{\partial \boldsymbol{r}^{*}}\left(\frac{\partial \Lambda^{\prime *}}{\partial \frac{\partial \varphi^{*}}{\partial \boldsymbol{r}^{*}}} \delta g_{i k}^{*}\right)-\frac{\partial}{\partial \boldsymbol{r}^{*}}\left(\frac{\partial \Lambda^{\prime *}}{\partial \frac{\partial \varphi^{*}}{\partial \boldsymbol{r}^{*}}}\right) \delta g_{i k^{*}}\right\}\right. \\
& \left.+\left\{\frac{\partial}{\partial t}\left(\frac{\partial \Lambda^{\prime *}}{\partial \frac{\partial g_{i k}^{*}}{\partial \boldsymbol{r}^{*}}} \delta g_{i k}{ }^{*}\right)-\frac{\partial}{\partial t}\left(\frac{\partial \Lambda^{\prime *}}{\partial \frac{\partial g_{i k}^{*}}{\partial t}}\right) \delta g_{i k}{ }^{*}\right\}\right] d V^{*} d t
\end{aligned}
$$




$$
\begin{aligned}
=\int\left[\int \int \left\{\frac{\partial}{\partial \boldsymbol{r}^{*}}(\right.\right. & \left.\left.\frac{\partial \Lambda^{\prime *}}{\partial \frac{\partial \varphi^{*}}{\partial \boldsymbol{r}^{*}}} \delta g_{i k}^{*}\right)+\frac{\partial}{\partial t}\left(\frac{\partial \Lambda^{\prime *}}{\partial \frac{\partial \varphi^{*}}{\partial \boldsymbol{r}^{*}}} \delta g_{i k}^{*}\right)\right\} \\
& \left.+\delta g_{i k}^{*}\left\{\frac{\partial \Lambda^{\prime *}}{\partial g_{i k^{*}}}-\frac{\partial}{\partial \boldsymbol{r}^{*}}\left(\frac{\partial \Lambda^{\prime *}}{\partial \frac{\partial g_{i k}^{*}}{\partial \boldsymbol{r}^{*}}}\right)-\frac{\partial}{\partial t}\left(\frac{\partial \Lambda^{\prime *}}{\partial \frac{\partial g_{i k}{ }^{*}}{\partial t}}\right)\right)\right] d V^{*} d t
\end{aligned}
$$

The least action principle tells us that

$$
\delta S\left[\left\{g_{i k}(x, t)\right\}\right]=0
$$

$=>\frac{\partial}{\partial t}\left(\frac{\partial \Lambda^{\prime *}}{\partial \frac{\partial g_{i k}{ }^{*}}{\partial t}}\right)+\frac{\partial}{\partial \boldsymbol{r}^{*}}\left(\frac{\partial \Lambda^{\prime *}}{\partial \frac{\partial g_{i k}{ }^{*}}{\partial \boldsymbol{r}^{*}}}\right)=\frac{\partial \Lambda^{\prime *}}{\partial g_{i k}{ }^{*}}=\frac{1}{\gamma\left(\boldsymbol{V}_{\mathbf{C}}\right)} \frac{\partial \Lambda^{*}}{\partial g_{i k}{ }^{*}}$

$$
<=>\gamma \frac{\partial}{\partial t}\left(\frac{\partial \Lambda^{\prime *}}{\partial \frac{\partial g_{i k}{ }^{*}}{\partial t}}\right)+\gamma \frac{\partial}{\partial \boldsymbol{r}^{*}}\left(\frac{\partial \Lambda^{\prime *}}{\partial \frac{\partial g_{i k}{ }^{*}}{\partial \boldsymbol{r}^{*}}}\right)=\frac{\partial \Lambda^{*}}{\partial g_{i k^{*}}}
$$

$$
<=>\gamma \frac{\partial}{\partial t}\left(\frac{\partial \Lambda^{*}}{\partial\left(\frac{\partial g_{i k^{*}}}{\partial t^{*}}\right)}\right)+\gamma \frac{\partial}{\partial r^{*}}\left(\frac{\partial \Lambda^{\prime *}}{\partial \frac{\partial g_{i k^{*}}}{\partial r^{*}}}\right)=\frac{\partial \Lambda^{*}}{\partial g_{i k^{*}}} \text { since } \frac{\partial \Lambda^{*}}{\partial \frac{\partial g_{i k^{*}}}{\partial t}}=\frac{\partial \Lambda^{*}}{\partial\left(\frac{\partial g_{i k^{*}}}{\partial t^{*}}\right)}
$$

$<=>\gamma \frac{\partial}{\partial t^{*}}\left(\frac{\partial \Lambda^{*}}{\partial\left(\frac{\partial g_{i k}^{*}}{\partial t^{*}}\right)}\right)+\gamma \frac{\partial}{\partial \boldsymbol{r}^{*}}\left(\frac{\partial \Lambda^{\prime *}}{\partial \frac{\partial g_{i k^{*}}}{\partial \boldsymbol{r}^{*}}}\right)=\frac{\partial \Lambda^{*}}{\partial g_{i k}{ }^{*}}$

$$
\text { Moreover } \frac{\partial}{\partial \boldsymbol{r}^{*}}\left(\frac{\partial \Lambda^{\prime *}}{\partial \frac{\partial g_{i k^{*}}}{\partial \boldsymbol{r}^{*}}}\right)=\frac{\partial}{\partial \boldsymbol{r}^{*}}\left(\frac{\left.\partial \frac{\Lambda^{*}\left(\varphi^{*}, \frac{\partial g_{i k}{ }^{*}}{\partial \boldsymbol{r}^{*}}, \gamma \frac{\partial g_{i k}{ }^{*}}{\partial t}\right)}{\gamma}\right)=\frac{1}{\gamma\left(\boldsymbol{V}_{\mathbf{C}}\right)} \frac{\partial}{\partial \boldsymbol{r}^{*}}\left(\frac{\partial \Lambda^{*}}{\partial \boldsymbol{r}^{*}}\right.}{\partial \frac{\partial g_{i k}}{\partial \boldsymbol{r}^{*}}}\right)
$$

And $\gamma\left(\boldsymbol{V}_{\mathbf{C}}\right) \frac{\partial}{\partial t}\left(\frac{\partial \Lambda^{*}}{\partial\left(\frac{\partial g_{i k^{*}}}{\partial t^{*}}\right)}\right)=\frac{\partial}{\partial t^{*}}\left(\frac{\partial \Lambda^{*}}{\partial\left(\frac{\partial g_{i k^{*}}}{\partial t^{*}}\right)}\right)$

$=>\frac{\partial}{\partial t^{*}}\left(\frac{\partial \Lambda^{*}}{\partial\left(\frac{\partial g_{i k}^{*}}{\partial t^{*}}\right)}\right)+\frac{\partial}{\partial \boldsymbol{r}^{*}}\left(\frac{\partial \Lambda^{*}}{\partial \frac{\partial g_{i k}^{*}}{\partial \boldsymbol{r}^{*}}}\right)=\frac{\partial \Lambda^{*}}{\partial g_{i k^{*}}}$ 


\section{Interaction between a field and a particle}

We consider the simplified action:

$$
S\left[\boldsymbol{r}_{\boldsymbol{a}}(t),\{\varphi(x, t)\}\right]=\int_{t_{1}}^{t_{2}}\left(\sum_{a}\left[-m_{a} \cdot c \frac{d s_{a}}{d t}-\frac{e_{a}}{c} \cdot \frac{d s_{a}}{d t} \varphi\left(\boldsymbol{r}_{\boldsymbol{a}}, t\right)\right]\right) d t+\frac{1}{c} \iiint \int \Lambda\left(\varphi, \frac{\partial \varphi}{\partial \boldsymbol{r}}, \frac{\partial \varphi}{\partial t}\right) d \Omega
$$

So we have also:

$$
\begin{aligned}
& S=\int_{t_{1}}^{t_{2}}\left(\sum_{a}\left[-\left(m_{a}+\frac{e_{a}}{c^{2}} \varphi\left(\boldsymbol{r}_{\boldsymbol{a}}, t\right)\right) \cdot c \cdot \frac{d s_{a}}{d t}\right]\right) d t+\frac{1}{c} \iiint \int \Lambda\left(\varphi, \frac{\partial \varphi}{\partial \boldsymbol{r}}, \frac{\partial \varphi}{\partial t}\right) d \Omega \\
& =\int_{t_{1}}^{t_{2}}\left(\sum_{a}\left[-\left(m_{a}+\frac{e_{a}}{c^{2}} \varphi^{K_{m}}\right) \cdot c^{2} \frac{1}{\gamma_{a}^{K_{m}}}\right]\right) \frac{d t}{\gamma\left(\boldsymbol{V}_{\mathbf{c}_{m}}\right)} \\
& +\int\left[\iiint \Lambda^{K_{\varphi}}\left(\varphi^{K_{\varphi}}, \frac{\partial \varphi^{K_{\varphi}}}{\partial r^{K_{\varphi}}}, \gamma\left(\boldsymbol{V}_{\mathbf{C}_{\varphi}}\right) \frac{\partial \varphi^{K_{\varphi}}}{\partial t}, \boldsymbol{R}_{\left.\left.\mathbf{C}_{\varphi}, \boldsymbol{V}_{\mathbf{C}_{\varphi}}\right) d V^{K_{\varphi}}\right]}\right] \frac{d t}{\gamma\left(\boldsymbol{V}_{\mathbf{C}_{\varphi}}\right)}\right.
\end{aligned}
$$

Where we have specified the quantities relative to:

- the frame $K_{\varphi}$ of the center of mass $\mathbf{C}_{\varphi}$ of the field $\varphi$;

- the frame $K_{m}$ of the center of mass $\mathbf{C}_{m}$ of the material system.

$$
\begin{aligned}
S\left[\left\{\boldsymbol{r}_{\boldsymbol{a}}^{K_{m}}\left(t^{K_{m}}\right)\right\},\right. & \left.\boldsymbol{R}_{\mathbf{C}_{m}}(t),\left\{\varphi^{K_{\varphi}}\left(x^{K_{\varphi}}, t^{K_{\varphi}}\right)\right\}, \boldsymbol{R}_{\mathbf{C}_{\varphi}}(\boldsymbol{t})\right] \\
& =\int_{t_{1}}^{t_{2}} L^{\prime}\left(\left\{\boldsymbol{r}_{\boldsymbol{a}}^{K_{m}}\right\},\left\{\frac{d \boldsymbol{r}_{\boldsymbol{a}}^{K_{m}}}{d t}\right\}, \boldsymbol{R}_{\mathbf{C}_{m}}, \boldsymbol{V}_{\mathbf{C}_{m}}, t\right) d t \\
& +\int L^{\prime}\left[\left\{\varphi^{K_{\varphi}}\right\},\left\{\frac{\partial \varphi^{K_{\varphi}}}{\partial \boldsymbol{r}^{K_{\varphi}}}\right\},\left\{\frac{\partial \varphi^{K_{\varphi}}}{\partial t}\right\}, \boldsymbol{R}_{\mathbf{C}_{\varphi}}, \boldsymbol{V}_{\mathbf{C}_{\varphi}}\right] d t
\end{aligned}
$$

So in this form, we can calculate the dynamic of the center of mass of one system and the other. We can see that each system is not free at all, but we have again:

$$
\begin{aligned}
& \boldsymbol{P}_{\mathbf{c}_{m}}=\gamma\left(\boldsymbol{V}_{\mathbf{c}_{m}}\right) \frac{E^{K_{m}}}{c^{2}} \boldsymbol{V}_{\mathbf{c}_{m}} \\
& \boldsymbol{P}_{\mathbf{c}_{\varphi}}=\gamma\left(\boldsymbol{V}_{\mathbf{c}_{\varphi}}\right) \frac{E^{K_{\varphi}}}{c^{2}} \boldsymbol{V}_{\mathbf{c}_{\varphi}}
\end{aligned}
$$

$$
\text { So } M_{m}=\frac{E^{K m}}{c^{2}}, M_{\varphi}=\frac{E^{K \varphi}}{c^{2}}
$$

With the same method we can consider any set of systems. 


\section{Does the mass of a body depend on the indeterminacy of the origin of energy?}

We have showed the generality of the Einstein law (without Energy-Momentum tensor). A question frequently come in mind when we derive this law is (see [1]) :

- the accordance between a characteristic quantity of an (apparent) particle, the mass M;

- and the a priori indeterminacy of the origin of the internal energy which is linked.

How to reconcile the 2 different aspects of theses quantities?

Moreover, in [1] it is stated that the mass sets the origin of the energy [scale] in Relativity, what does that mean?

\subsection{A free material system}

As above we start from the Least Action Principle:

$$
S\left[\left\{\boldsymbol{r}_{\boldsymbol{a}}(t)\right\}\right]=\int_{t_{1}}^{t_{2}} L\left(\left\{\boldsymbol{r}_{\boldsymbol{a}}\right\},\left\{\frac{d \boldsymbol{r}_{\boldsymbol{a}}}{d t}\right\}\right) d t
$$

And again:

$$
S\left[\left\{\boldsymbol{r}_{\boldsymbol{a}}^{*}\left(t^{*}\right), \boldsymbol{R}_{\boldsymbol{c}}(t)\right\}\right]=\int_{\left\{\boldsymbol{t}_{\boldsymbol{a}, \mathbf{1}}^{*}\right\}}^{\left\{\boldsymbol{t}_{\boldsymbol{a}, 2}^{*}\right\}} L^{*}\left(\left\{\boldsymbol{r}_{\boldsymbol{a}}^{*}\right\},\left\{\frac{d \boldsymbol{r}_{\boldsymbol{a}}^{*}}{d t^{*}}\right\}\right) d t^{*}
$$

Taking account $d t^{*}=\frac{d t}{\gamma(t)}$ and returnig to the Galilean frame $\mathrm{K}$ we have:

$$
S=\int_{\left\{\boldsymbol{t}_{\boldsymbol{a}, \mathbf{1}}^{*}\right\}}^{\left\{\boldsymbol{t}_{\boldsymbol{a}, 2}^{*}\right\}} L^{*}\left(\left\{\boldsymbol{r}_{\boldsymbol{a}}^{*}\right\},\left\{\frac{d t}{d t^{*}} \frac{d \boldsymbol{r}_{\boldsymbol{a}}^{*}}{d t}\right\}\right) \frac{d t^{*}}{d t} d t=\int_{t_{1}}^{t_{2}} \frac{L^{*}\left(\left\{\boldsymbol{r}_{\boldsymbol{a}}^{*}\right\},\left\{\gamma\left(\boldsymbol{V}_{\mathbf{C}}\right) \frac{d \boldsymbol{r}_{\boldsymbol{a}}^{*}}{d t}\right\}\right)}{\gamma\left(\boldsymbol{V}_{\mathbf{C}}\right)} d t
$$

We already know that $\boldsymbol{P}_{\boldsymbol{c}}=\frac{\partial}{\partial v_{\mathrm{C}}} \frac{L^{*}\left(\left\{r_{\boldsymbol{a}}^{*}\right\},\left\{\gamma\left(v_{\mathrm{C}}\right) \frac{\left.d r_{a}^{*}\right\}}{d t}\right\}\right)}{\gamma\left(\boldsymbol{v}_{\mathrm{C}}\right)}=\gamma\left(\boldsymbol{V}_{\mathrm{C}}\right) \frac{E^{*}}{c^{2}} \boldsymbol{V}_{\mathrm{C}}=\gamma\left(\boldsymbol{V}_{\mathbf{C}}\right) M \boldsymbol{V}_{\mathrm{C}}$

Thanks to the indeterminacy of the action we can also physically work with the equivalent action:

$$
S_{\text {modif }}\left[\left\{\boldsymbol{r}_{\boldsymbol{a}}(t)\right\}\right]=\int_{t_{1}}^{t_{2}} L_{\text {modif }}\left(\left\{\boldsymbol{r}_{\boldsymbol{a}}\right\},\left\{\frac{d \boldsymbol{r}_{\boldsymbol{a}}}{d t}\right\}\right) d t=\int_{t_{1}}^{t_{2}}\left(L\left(\left\{\boldsymbol{r}_{\boldsymbol{a}}\right\},\left\{\frac{d \boldsymbol{r}_{\boldsymbol{a}}}{d t}\right\}\right)+\frac{d f\left(t,\left\{\boldsymbol{r}_{\boldsymbol{a}}\right\}\right)}{d t}\right) d t
$$

Which gives now:

$$
\begin{aligned}
& S_{\text {modif }}\left[\left\{\boldsymbol{r}_{\boldsymbol{a}}^{*}\left(t^{*}\right), \boldsymbol{R}_{\boldsymbol{c}}(t)\right\}\right]=\int_{\left\{\boldsymbol{t}_{\boldsymbol{a}, \mathbf{1}}^{*}\right\}}^{\left\{\boldsymbol{t}_{\boldsymbol{a}, \boldsymbol{2}}^{*}\right\}}\left(L^{*}\left(\left\{\boldsymbol{r}_{\boldsymbol{a}}^{*}\right\},\left\{\frac{d \boldsymbol{r}_{\boldsymbol{a}}^{*}}{d t^{*}}\right\}\right)+\frac{d f\left(t^{*},\left\{\boldsymbol{r}_{\boldsymbol{a}}^{*}\right\}\right)}{d t^{*}}\right) d t^{*} \\
& =\int_{\left\{\boldsymbol{t}_{\boldsymbol{a}, \mathbf{1}}^{*}\right\}}^{\left\{\boldsymbol{t}_{\boldsymbol{a}, \boldsymbol{2}}^{*}\right\}}\left(L^{*}\left(\left\{\boldsymbol{r}_{\boldsymbol{a}}^{*}\right\},\left\{\frac{d \boldsymbol{r}_{\boldsymbol{a}}^{*}}{d t^{*}}\right\}\right)+\frac{d f\left(t^{*},\left\{\boldsymbol{r}_{\boldsymbol{a}}^{*}\right\}\right)}{d t^{*}}\right) \frac{d t^{*}}{d t} d t \\
& =\int_{t_{1}}^{t_{2}} \frac{L^{*}\left(\left\{\boldsymbol{r}_{\boldsymbol{a}}^{*}\right\},\left\{\frac{d \boldsymbol{r}_{\boldsymbol{a}}^{*}}{d t^{*}}\right\}\right)+\frac{d f\left(t^{*},\left\{\boldsymbol{r}_{\boldsymbol{a}}^{*}\right\}\right)}{d t^{*}}}{\gamma\left(\boldsymbol{V}_{\mathbf{C}}\right)} d t
\end{aligned}
$$

So we can work with the modified Lagrangian: 
$L_{\text {modif }}^{\prime}\left(\left\{\boldsymbol{r}_{\boldsymbol{a}}^{*}\right\},\left\{\frac{d \boldsymbol{r}_{\boldsymbol{a}}^{*}}{d t}\right\}, \boldsymbol{R}_{\mathbf{C}}, \boldsymbol{V}_{\mathbf{C}}\right)=\frac{L^{*}\left(\left\{\boldsymbol{r}_{\boldsymbol{a}}^{*}\right\},\left\{\frac{d \boldsymbol{r}_{\boldsymbol{a}}^{*}}{d t^{*}}\right\}\right)+\gamma\left(\boldsymbol{V}_{\mathbf{C}}\right) \frac{d f\left(t^{*},\left\{\boldsymbol{r}_{\boldsymbol{a}}^{*}\right\}\right)}{d t}}{\gamma\left(\boldsymbol{V}_{\mathbf{C}}\right)}$

$$
=\frac{L^{*}\left(\left\{\boldsymbol{r}_{\boldsymbol{a}}^{*}\right\},\left\{\frac{d \boldsymbol{r}_{\boldsymbol{a}}^{*}}{d t^{*}}\right\}\right)}{\gamma\left(\boldsymbol{V}_{\mathbf{C}}\right)}+\frac{d f\left(t^{*},\left\{\boldsymbol{r}_{\boldsymbol{a}}^{*}\right\}\right)}{d t}
$$

But $\quad \frac{d f\left(t^{*},\left\{r_{a}^{*}\right\}\right)}{d t}=\frac{\sum a \frac{\partial f\left(x_{\left.j, a^{*}\right)}\right.}{\partial x_{i, a^{*}}} d x_{i, a^{*}}}{d t}=\sum_{a} \frac{\partial f\left(t^{*},\left\{\boldsymbol{r}_{a}^{*}\right\}\right)}{c . \partial t^{*}} c \frac{d t^{*}}{d t}+\frac{\partial f\left(t^{*},\left\{\boldsymbol{r}_{a}^{*}\right\}\right)}{\partial \boldsymbol{r}_{\boldsymbol{a}}^{*}} \frac{d \boldsymbol{r}_{a}^{*}}{d t}=$

$$
\begin{gathered}
=\sum_{a} \frac{1}{\gamma} \frac{\partial f\left(t^{*},\left\{\boldsymbol{r}_{\boldsymbol{a}}^{*}\right\}\right)}{\partial t^{*}}+\frac{\partial f\left(t^{*},\left\{\boldsymbol{r}_{\boldsymbol{a}}^{*}\right\}\right)}{\partial \boldsymbol{r}_{\boldsymbol{a}}^{*}} \frac{d \boldsymbol{r}_{\boldsymbol{a}}^{*}}{d t} \\
=>\frac{\partial}{\partial \boldsymbol{V}_{\mathbf{C}}} \frac{d f\left(t^{*},\left\{\boldsymbol{r}_{\boldsymbol{a}}^{*}\right\}\right)}{d t}=\left(\sum_{a} \frac{\partial f\left(t^{*},\left\{\boldsymbol{r}_{\boldsymbol{a}}^{*}\right\}\right)}{\partial t^{*}}\right) \frac{\partial}{\partial \boldsymbol{v}_{\mathbf{c}}} \frac{1}{\gamma}=\left(\sum_{a} \frac{\partial f\left(t^{*},\left\{\boldsymbol{r}_{\boldsymbol{a}}^{*}\right\}\right)}{\partial t^{*}}\right)\left(-\gamma\left(\boldsymbol{V}_{\mathbf{C}}\right) \frac{\boldsymbol{V}_{\mathbf{C}}}{\boldsymbol{c}^{2}}\right)
\end{gathered}
$$

since $\frac{\partial}{\partial v_{\mathrm{c}}} \frac{1}{\gamma\left(v_{\mathrm{c}}\right)}=-\gamma\left(V_{\mathrm{C}}\right) \frac{v_{\mathrm{C}}}{c^{2}}$

We see that:

$$
\boldsymbol{P}_{\boldsymbol{c}}=\frac{\partial L_{\text {modif }}^{\prime}}{\partial \boldsymbol{v}_{\mathbf{C}}}=\frac{\partial L^{\prime}}{\partial \boldsymbol{v}_{\mathbf{C}}}-\frac{\gamma\left(\boldsymbol{V}_{\mathbf{C}}\right)\left(\sum_{a} \frac{\partial f\left(t^{*},\left\{\boldsymbol{r}_{\boldsymbol{a}}^{*}\right\}\right)}{\partial t^{*}}\right)}{c^{2}} \boldsymbol{V}_{\mathbf{C}}=\gamma\left(\boldsymbol{V}_{\mathbf{C}}\right)\left(M-\frac{1}{c^{2}} \sum_{a} \frac{\partial f\left(t^{*},\left\{\boldsymbol{r}_{\boldsymbol{a}}^{*}\right\}\right)}{\partial t^{*}}\right) \boldsymbol{V}_{\mathbf{C}}
$$

Hence, the mass is indeed a priori indeterminate as :

- the general mass has the form $M_{\text {modif }}=M-\frac{1}{c^{2}} \sum_{a} \frac{\partial f\left(t^{*},\left\{r_{r}^{*}\right\}\right)}{\partial t^{*}}$;

- with $f\left(t^{*},\left\{\boldsymbol{r}_{\boldsymbol{a}}^{*}\right\}\right)$ a function which can be freely chosen.

Yet, there is a point that we have neglected so far: the relativistic invariance.

We can always make the choice, permitted in Relativity, to consider only the expression in the Lagrangian which gives a relativistic invariant. This restrict us to choose $f\left(t^{*},\left\{\boldsymbol{r}_{\boldsymbol{a}}^{*}\right\}\right)=0$, as the only relativistic invariant associated to free a particle is the 4D line element $d s$.

$$
\left(M_{\text {modif }}\right)_{L_{a} d t \text { is chose relativistic invariant }}=M-\frac{1}{c^{2}} \sum_{a} \frac{\partial 0}{\partial t^{*}}=M
$$

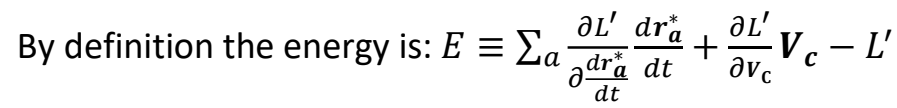

$$
\begin{aligned}
& E_{\text {modif }} \equiv \sum_{a} \frac{\partial L_{\text {modif }}{ }^{\prime}}{\partial \frac{d \boldsymbol{r}_{\boldsymbol{a}}^{*}}{d t}} \frac{d \boldsymbol{r}_{\boldsymbol{a}}^{*}}{d t}+\frac{\partial L_{\text {modif }}{ }^{\prime}}{\partial \boldsymbol{V}_{\mathrm{C}}} \boldsymbol{V}_{\boldsymbol{c}}-L_{\text {modif }}{ }^{\prime} \\
& =\sum_{a} \frac{\partial\left(\frac{L^{*}\left(\left\{\boldsymbol{r}_{a}^{*}\right\},\left\{\frac{d \boldsymbol{r}_{a}^{*}}{\left.d t^{*}\right\}}\right.\right.}{\gamma\left(\boldsymbol{V}_{\mathrm{C}}\right)}+\frac{d f\left(t^{*},\left\{\boldsymbol{r}_{a}^{*}\right\}\right)}{d t}\right)}{\partial \frac{d \boldsymbol{r}_{a}^{*}}{d t}} \frac{d \boldsymbol{r}_{a}^{*}}{d t}+\frac{\partial\left(\frac{L^{*}\left(\left\{\boldsymbol{r}_{a}^{*}\right\},\left\{\frac{d \boldsymbol{r}_{a}^{*}}{\left.d t^{*}\right\}}\right)\right.}{\gamma\left(\boldsymbol{V}_{\mathrm{C}}\right)}+\frac{d f\left(t^{*},\left\{\boldsymbol{r}_{a}^{*}\right\}\right)}{d t}\right)}{\partial \boldsymbol{V}_{\mathbf{C}}} \boldsymbol{V}_{\boldsymbol{c}}-L_{\text {modif }}{ }^{\prime}
\end{aligned}
$$




$$
\begin{aligned}
& =\sum_{a} \frac{\partial\left(\frac{L^{*}\left(\left\{\boldsymbol{r}_{a}^{*}\right\},\left\{\frac{d \boldsymbol{r}_{a}^{*}}{\left.d t^{*}\right\}}\right)\right.}{\gamma\left(\boldsymbol{V}_{\mathrm{c}}\right)}+\frac{d f\left(t^{*},\left\{\boldsymbol{r}_{a}^{*}\right\}\right)}{d t}\right)}{\partial \frac{d \boldsymbol{r}_{a}^{*}}{d t}} \frac{d \boldsymbol{r}_{a}^{*}}{d t}+\frac{\partial\left(\frac{L^{*}\left(\left\{\boldsymbol{r}_{a}^{*}\right\},\left\{\frac{d \boldsymbol{r}_{a}^{*}}{\left.d t^{*}\right\}}\right)\right.}{\gamma\left(\boldsymbol{V}_{\mathrm{C}}\right)}+\frac{d f\left(t^{*},\left\{\boldsymbol{r}_{a}^{*}\right\}\right)}{d t}\right)}{\partial \boldsymbol{V}_{\mathrm{C}}} \boldsymbol{V}_{\boldsymbol{c}}-\left(\frac{L^{*}\left(\left\{\boldsymbol{r}_{a}^{*}\right\},\left\{\frac{d \boldsymbol{r}_{a}^{*}}{d t^{*}}\right\}\right)}{\gamma\left(\boldsymbol{V}_{\mathrm{C}}\right)}+\frac{d f\left(t^{*},\left\{\boldsymbol{r}_{a}^{*}\right\}\right)}{d t}\right)
\end{aligned}
$$

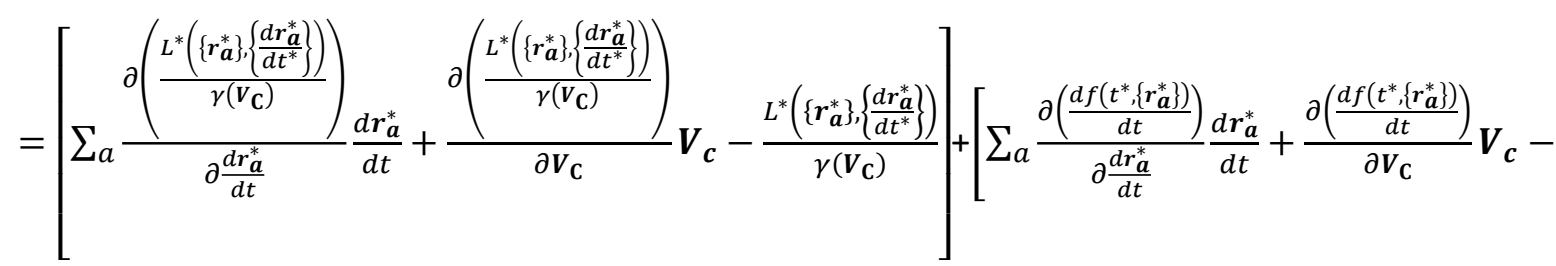

$$
\begin{aligned}
& \left.\frac{d f\left(t^{*},\left\{r_{a}^{*}\right\}\right)}{d t}\right] \\
& =\gamma E^{*}+\sum_{a} \frac{\partial\left(\frac{d f\left(t^{*},\left\{r_{a}^{*}\right\}\right)}{d t}\right)}{\partial \frac{d r_{a}^{*}}{d t}} \frac{d r_{a}^{*}}{d t}+\frac{\partial\left(\frac{d f\left(t^{*},\left\{r_{a}^{*}\right\}\right)}{d t}\right)}{\partial V_{\mathbf{C}}} \boldsymbol{V}_{\boldsymbol{c}}-\frac{d f\left(t^{*},\left\{r_{a}^{*}\right\}\right)}{d t} \\
& =\gamma E^{*}+\sum_{a}\left(\frac{\partial\left(\sum a_{\gamma} \frac{1 \partial f\left(t^{*},\left\{r_{a}^{*}\right\}\right)}{\partial t^{*}}+\frac{\partial f\left(t^{*},\left\{r_{a}^{*}\right\}\right) d r_{a}^{*}}{\partial t}\right)}{\partial \frac{d r_{a}^{*}}{d t}}\right) \frac{d r_{a}^{*}}{d t}+\frac{\partial\left(\sum_{a} \frac{1 \partial f\left(t^{*},\left\{r_{*}^{*}\right\}\right)}{\partial t^{*}}+\frac{\partial f\left(t^{*},\left\{*_{a}^{*}\right\}\right) d r_{a}^{*}}{\partial r_{a}^{*}} d t\right)}{\partial \boldsymbol{V}_{\mathrm{C}}} \boldsymbol{V}_{\boldsymbol{c}}-\frac{d f\left(t^{*},\left\{\boldsymbol{r}_{a}^{*}\right\}\right)}{d t} \\
& =\gamma E^{*}+\sum_{a} \frac{\partial f\left(t^{*},\left\{r_{a}^{*}\right\}\right)}{\partial r_{a}^{*}} \frac{d r_{a}^{*}}{d t}+\sum_{a} \frac{\partial f\left(t^{*},\left\{r_{a}^{*}\right\}\right)}{\partial t^{*}} \frac{\partial\left(\frac{1}{\gamma}\right)}{\partial V_{\mathbf{C}}} V_{c}-\frac{d f\left(t^{*},\left\{r_{a}^{*}\right\}\right)}{d t} \\
& =\gamma E^{*}+\sum_{a} \frac{\partial f\left(t^{*},\left\{\boldsymbol{r}_{\boldsymbol{a}}^{*}\right\}\right)}{\partial \boldsymbol{r}_{\boldsymbol{a}}^{*}} \frac{d \boldsymbol{r}_{a}^{*}}{d t}+\sum_{a} \frac{\partial f\left(t^{*},\left\{\boldsymbol{r}_{a}^{*}\right\}\right)}{\partial t^{*}}\left(-\gamma\left(\boldsymbol{V}_{\mathbf{C}}\right) \frac{\boldsymbol{V}_{\mathbf{c}}}{\boldsymbol{c}^{2}}\right) \boldsymbol{V}_{\boldsymbol{c}}-\frac{d f\left(t^{*},\left\{\boldsymbol{r}_{a}^{*}\right\}\right)}{d t} \\
& =\gamma E^{*}+\sum_{a} \frac{\partial f\left(t^{*},\left\{\boldsymbol{r}_{a}^{*}\right\}\right)}{\partial \boldsymbol{r}_{\boldsymbol{a}}^{*}} \frac{d \boldsymbol{r}_{a}^{*}}{d t}-\sum_{a} \frac{\partial f\left(t^{*},\left\{\boldsymbol{r}_{a}^{*}\right\}\right)}{\partial t^{*}} \gamma\left(\boldsymbol{V}_{\mathrm{C}}\right) \frac{\boldsymbol{V}_{\mathrm{c}}{ }^{2}}{c^{2}}-\left(\sum_{a} \frac{1}{\gamma} \frac{\partial f\left(t^{*},\left\{\boldsymbol{r}_{a}^{*}\right\}\right)}{\partial t^{*}}+\frac{\partial f\left(t^{*},\left\{\boldsymbol{r}_{a}^{*}\right\}\right)}{\partial \boldsymbol{r}_{\boldsymbol{a}}^{*}} \frac{d \boldsymbol{r}_{a}^{*}}{d t}\right) \\
& =\gamma E^{*}-\sum_{a} \frac{\partial f\left(t^{*},\left\{\boldsymbol{r}_{a}^{*}\right\}\right)}{\partial t^{*}} \gamma\left(\boldsymbol{V}_{\mathbf{C}}\right) \frac{\boldsymbol{V}_{\mathbf{C}}{ }^{2}}{c^{2}}-\left(\sum_{a} \frac{1}{\gamma} \frac{\partial f\left(t^{*},\left\{\boldsymbol{r}_{a}^{*}\right\}\right)}{\partial t^{*}}\right) \\
& =\gamma E^{*}-\sum_{a} \frac{\partial f\left(t^{*},\left\{\boldsymbol{r}_{a}^{*}\right\}\right)}{\partial t^{*}}\left[\frac{1+\gamma^{2} \beta^{2}}{\gamma}\right]=\gamma E^{*}-\sum_{a} \frac{\partial f\left(t^{*},\left\{\boldsymbol{r}_{a}^{*}\right\}\right)}{\partial t^{*}}\left[\frac{\frac{1-\beta^{2}+\beta^{2}}{1-\beta^{2}}}{\gamma}\right]=\gamma E^{*}-\gamma \sum_{a} \frac{\partial f\left(t^{*},\left\{\boldsymbol{r}_{a}^{*}\right\}\right)}{\partial t^{*}} \\
& E_{\text {modif }}=\gamma\left(E^{*}-\sum_{a} \frac{\partial f\left(t^{*},\left\{\boldsymbol{r}_{a}^{*}\right\}\right)}{\partial t^{*}}\right)
\end{aligned}
$$

But we know that $M_{\text {modif }}=M-\frac{1}{c^{2}} \sum_{a} \frac{\partial f\left(t^{*},\left\{r_{a}^{*}\right\}\right)}{\partial t^{*}}$

Thus we can also write

$$
E_{\text {modif }}=\gamma M_{\text {modif }} c^{2}
$$

We see that the origin of the Energy scale is the mass, even if the mass is modified by the indeterminacy of the Lagrangian.

It results also from that, the requirement to working only with relativistic invariant "Lagrangian" L.dt, sets the value of the mass (by implying $f\left(t^{*},\left\{\boldsymbol{r}_{\boldsymbol{a}}^{*}\right\}\right)=\frac{1}{c^{2}} \sum_{a} \frac{\partial f\left(t^{*},\left\{\boldsymbol{r}_{a}^{*}\right\}\right)}{\partial t^{*}}=0$ ) and consequently sets the origin of the scale of the total Energy, at a non arbitrary value. 
$\underline{\text { Relativistic invariance in conjunction with Lagrangian mechanic }}=>$

- $=>\frac{1}{c^{2}} \sum_{a} \frac{\partial f\left(t^{*},\left\{r_{a}^{*}\right\}\right)}{\partial t^{*}}=0$

- $=>E_{\text {modif }}=E \& M_{\text {modif }}=M$

- $\quad=>E=\gamma\left(\boldsymbol{V}_{\boldsymbol{c}}\right) M c^{2}$

- $\quad=>$ "The origin of the Energy scale is fixed by the mass [1] \& at a non arbitrary value":

If we put simply $\boldsymbol{V}_{\boldsymbol{c}}=\mathbf{0}$ we have $E_{0}=M c^{2}$.

Indeed, like Landau-Lifchitz [01] we can effectively say that in Special Relativity, the origin of the Energy scale is fixed by the mass (at least for free material system): If we put simply $\boldsymbol{V}_{\boldsymbol{c}}=\mathbf{0}$ we have $E_{0}=M c^{2}$.

Another point. In textbook we give easily to proof for the formula $E=\gamma\left(\boldsymbol{V}_{\boldsymbol{c}}\right) M c^{2}$. Starting from this :

- we compute what we call the rest energy $E_{0} \equiv(E)_{V_{c}=0}=M c^{2}$.

- Then we say "The mass is the Energy at rest", having actually in mind that this rest energy $E_{0}$

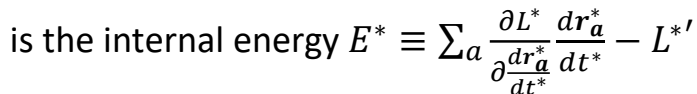

- But...where is the proof of $E_{0}=E^{*}$ ? Without an analysis à la Landau-Lifchitz [1] using the energy-momentum tensor this affirmation has no foundation. In this article I have given another equivalent proof and I think more easy to understand (Einstein himself was helped by Klein in order to improve his proof using the energy-momentum tensor, indeed he failed to give a totally general one, cf. [7], despite his intuitively convincing different demonstrations).

It is not at all a trivial statement as the way to prove it was not so easy, even if the elementary relativistic formula permit us indeed to guess it. But to guess is not to prove, that is to say to totally understand. 


\subsection{A material system in an external Electromagnetic field}

\section{- The momentum \& the mass for material system in an external Electromagnetic field}

Although the relativistic invariance gives a clear criteria to set the mass of a free material system, the same material system seen in external field (electromagnetic field, gravitational field) has its origin broken by other kind of invariance of physics law (in point of view of the least action principle): gauge invariance in Electromagnetism \& al, transformation of coordinate system in General Relativity.

In the case of electromagnetism the action of a particle in a given 4-potential is ([1]):

$$
\begin{gathered}
S\left[\left\{\boldsymbol{r}_{\boldsymbol{a}}(t)\right\}, t\right]=\int_{s_{1}}^{s_{2}} \sum_{a}\left[-m_{a} \cdot c d s_{a}-\frac{e_{a}}{c} \cdot A^{i}\left(x_{a i}\right) d x_{a i}\right] \\
=\int_{t_{1}}^{t_{2}} \sum_{a}\left[-m_{a} \cdot c \frac{d s_{a}}{d t}-\frac{e_{a}}{c} \cdot A^{i}\left(x_{a i}\right) \frac{d x_{a i}}{d t}\right] d t \\
=>L\left(\left\{\boldsymbol{r}_{\boldsymbol{a}}(t)\right\},\left\{\frac{d \boldsymbol{r}_{\boldsymbol{a}}}{d t}\right\}, t\right)=\sum_{a}\left[-m_{a} \cdot c \frac{d s_{a}}{d t}-\frac{e_{a}}{c} \cdot A^{i}\left(x_{a i}\right) \frac{d x_{a i}}{d t}\right]
\end{gathered}
$$

Like above, we have also:

$$
\begin{aligned}
& S\left[\left\{\boldsymbol{r}_{\boldsymbol{a}}^{*}(t)\right\}, \boldsymbol{R}_{\mathbf{C}}(t)\right]=\int_{t_{1}}^{t_{1}}\left(\frac{\sum_{a}\left[-m_{a} \cdot c \frac{d s_{a}{ }^{*}}{d t^{*}}-\frac{e_{a}}{c} \cdot A^{i^{*}}\left(x_{a i}^{*}\right) \frac{d x_{a i}^{*}}{d t^{*}}\right]}{\gamma\left(\boldsymbol{V}_{\mathbf{C}}\right)}\right) d t \\
= & L^{\prime}\left(\left\{\boldsymbol{r}_{\boldsymbol{a}}^{*}(t)\right\},\left\{\frac{d \boldsymbol{r}_{\boldsymbol{a}}^{*}}{d t}\right\}, \boldsymbol{R}_{\mathbf{C}}, \boldsymbol{V}_{\mathbf{C}}, t\right)=\frac{\sum_{a}\left[-m_{a} \cdot c \frac{d s_{a}{ }^{*}}{d t^{*}}-\frac{e_{a}}{c} \cdot A^{i^{*}}\left(x_{a i}^{*}\right) \frac{d x_{a i}^{*}}{d t^{*}}\right]}{\gamma\left(\boldsymbol{V}_{\mathbf{C}}\right)}
\end{aligned}
$$

If we develop the expression

$$
\begin{aligned}
& L^{\prime}\left(\left\{\boldsymbol{r}_{\boldsymbol{a}}^{*}(t)\right\},\left\{\frac{d \boldsymbol{r}_{\boldsymbol{a}}^{*}}{d t}\right\}, \boldsymbol{R}_{\mathrm{C}}, \boldsymbol{V}_{\mathrm{C}}, t\right)=\frac{\sum_{a}\left[-m_{a} \cdot c^{2} \frac{d \tau_{a}^{*}}{d t^{*}}-\frac{e_{a}}{c} \cdot\left(\varphi^{*}\left(\boldsymbol{r}_{\boldsymbol{a}}^{*}, t^{*}\right) c \frac{d t^{*}}{d t^{*}}-\boldsymbol{A}^{*}\left(\boldsymbol{r}_{\boldsymbol{a}}^{*}, t^{*}\right) \frac{d \boldsymbol{r}_{\boldsymbol{a}}^{*}}{d t^{*}}\right)\right]}{\gamma\left(\boldsymbol{V}_{\mathrm{C}}\right)} \\
= & \frac{\sum_{a}\left[-\frac{m_{a} \cdot c^{2}}{\gamma_{a}{ }^{*}}-\frac{e_{a}}{c} \cdot\left(\varphi^{*}\left(\boldsymbol{r}_{\boldsymbol{a}}^{*}, t^{*}\right) c-\boldsymbol{A}^{*} \cdot \gamma\left(\boldsymbol{V}_{\mathrm{C}}\right) \frac{d \boldsymbol{r}_{\boldsymbol{a}}^{*}}{d t}\right)\right]}{\gamma\left(\boldsymbol{V}_{\mathrm{C}}\right)} \\
= & \frac{\sum_{a}\left[-\left(\frac{m_{a} \cdot c^{2}}{\gamma_{a}^{*}}+e_{a} \cdot \varphi^{*}\left(\boldsymbol{r}_{\boldsymbol{a}}^{*}, t^{*}\right)\right)+\frac{e_{a}}{c} \boldsymbol{A}^{*}\left(\boldsymbol{r}_{\boldsymbol{a}}^{*}, t^{*}\right) \cdot \gamma\left(\boldsymbol{V}_{\mathrm{C}}\right) \frac{d \boldsymbol{r}_{\boldsymbol{a}}^{*}}{d t}\right]}{\gamma\left(\boldsymbol{V}_{\mathrm{C}}\right)} \\
= & -\frac{\sum_{a}\left[\frac{m_{a} \cdot c^{2}}{\gamma_{a}^{*}}+e_{a} \cdot \varphi^{*}\left(\boldsymbol{r}_{\boldsymbol{a}}^{*}, t^{*}\right)\right]}{\gamma\left(\boldsymbol{V}_{\mathrm{C}}\right)}+\sum_{a} \frac{e_{a}}{c} \boldsymbol{A}^{*}\left(\boldsymbol{r}_{\boldsymbol{a}}^{*}, t^{*}\right) \cdot \frac{d \boldsymbol{r}_{\boldsymbol{a}}^{*}}{d t}
\end{aligned}
$$




$$
\begin{gathered}
=>L^{\prime}\left(\left\{\boldsymbol{r}_{\boldsymbol{a}}^{*}(t)\right\},\left\{\frac{d \boldsymbol{r}_{\boldsymbol{a}}^{*}}{d t}\right\}, \boldsymbol{R}_{\mathbf{C}}, \boldsymbol{V}_{\mathbf{C}}, t\right)=-\frac{\sum_{a}\left[\frac{m_{a} \cdot c^{2}}{\gamma_{a}{ }^{*}}+e_{a} \cdot \varphi^{*}\left(\boldsymbol{r}_{\boldsymbol{a}}^{*}, t^{*}\right)\right]}{\gamma\left(\boldsymbol{V}_{\mathbf{C}}\right)}+\sum_{a} \frac{e_{a}}{c} \boldsymbol{A}^{*}\left(\boldsymbol{r}_{\boldsymbol{a}}^{*}, t^{*}\right) \cdot \frac{d \boldsymbol{r}_{\boldsymbol{a}}^{*}}{d t} \\
=>\boldsymbol{P}_{\boldsymbol{c}}=\frac{\partial L^{\prime}}{\partial \boldsymbol{V}_{\mathbf{C}}}=\gamma\left(\boldsymbol{V}_{\mathbf{C}}\right)\left(\frac{\sum_{a}\left[\gamma_{a}^{*} \cdot m_{a} \cdot c^{2}+e_{a} \cdot \varphi^{*}\left(\boldsymbol{r}_{\boldsymbol{a}}^{*}, t^{*}\right)\right]}{c^{2}}\right) \boldsymbol{V}_{\mathbf{C}}
\end{gathered}
$$

Which gives a mass:

$$
\begin{gathered}
M=\frac{\sum_{a}\left[\gamma_{a}^{*} \cdot m_{a} \cdot c^{2}+e_{a} \cdot \varphi^{*}\left(\boldsymbol{r}_{a}^{*}, t^{*}\right)\right]}{c^{2}}=M_{\text {free }}+M_{\text {interaction }} \\
\text { With } M_{\text {free }} \equiv \frac{\sum_{a} \gamma_{a}^{*} \cdot m_{a} \cdot c^{2}}{c^{2}}=\sum_{a} \gamma_{a}^{*} \cdot m_{a} \\
M_{\text {interaction }} \equiv \frac{\sum_{a}\left[e_{a} \cdot \varphi^{*}\left(\boldsymbol{r}_{a}^{*}, t^{*}\right)\right]}{c^{2}}
\end{gathered}
$$

The gauge invariance permits us to write with the equivalent physical action:

$$
L_{\text {modif }}\left(\left\{\boldsymbol{r}_{\boldsymbol{a}}(t)\right\},\left\{\frac{d \boldsymbol{r}_{\boldsymbol{a}}}{d t}\right\}, t\right)=\sum_{a}\left[-m_{a} \cdot c \frac{d s_{a}}{d t}-\frac{e_{a}}{c} \cdot\left[A^{i}\left(x_{a i}\right)+\frac{\partial f\left(x_{a j}\right)}{\partial x_{a i}}\right] \frac{d x_{a i}}{d t}\right]
$$

If we now take account the possibility to change of the gauge

$$
\begin{aligned}
& L_{\text {motif }}^{\prime}\left(\left\{\boldsymbol{r}_{\boldsymbol{a}}^{*}(t)\right\},\left\{\frac{d \boldsymbol{r}_{\boldsymbol{a}}^{*}}{d t}\right\}, \boldsymbol{R}_{\mathbf{C}}, \boldsymbol{V}_{\mathbf{C}}, t\right)=\frac{\sum_{a}\left[-m_{a} \cdot c \frac{d s_{a}{ }^{*}}{d t^{*}}-\frac{e_{a}}{c} \cdot A_{\text {motif }}{ }^{i^{*}}\left(x_{a j}^{*}\right) \frac{d x_{a i}^{*}}{d t^{*}}\right]}{\gamma\left(\boldsymbol{V}_{\mathbf{C}}\right)} \\
& =\frac{\sum_{a}\left[-m_{a} \cdot c \frac{d s_{a}{ }^{*}}{d t^{*}}-\frac{e_{a}}{c} \cdot\left[A^{i^{*}}\left(x_{a j}^{*}\right)+\frac{\partial f\left(x_{a j}^{*}\right)}{\partial x_{a i}^{*}}\right] \frac{d x_{a i}^{*}}{d t^{*}}\right]}{\gamma\left(\boldsymbol{V}_{\mathbf{c}}\right)} \\
& =\frac{\sum_{a}\left[\left(-m_{a} \cdot c \frac{d s_{a}^{*}}{d t^{*}}-\frac{e_{a}}{c} \cdot A^{i^{*}}\left(x_{a j}^{*}\right) \frac{d x_{a i}^{*}}{d t^{*}}\right)-\frac{e_{a}}{c} \cdot \frac{\partial f\left(x_{a j}^{*}\right)}{\partial x_{a i}^{*}} \frac{d x_{a i}^{*}}{d t^{*}}\right]}{\gamma\left(\boldsymbol{V}_{\mathbf{C}}\right)} \\
& =\frac{\sum_{a}\left[\left(-\left(\frac{m_{a} \cdot c^{2}}{\gamma_{a}{ }^{*}}+e_{a} \cdot \varphi^{*}\left(\boldsymbol{r}_{\boldsymbol{a}}^{*}, t^{*}\right)\right)+\frac{e_{a}}{c} \boldsymbol{A}^{*}\left(\boldsymbol{r}_{\boldsymbol{a}}^{*}, t^{*}\right) \cdot \gamma\left(\boldsymbol{V}_{\mathrm{C}}\right) \frac{d \boldsymbol{r}_{\boldsymbol{a}}^{*}}{d t}\right)-\frac{e_{a}}{c} \cdot \frac{\partial f\left(x_{a j}^{*}\right)}{\partial x_{a i}^{*}} \frac{d x_{a i}^{*}}{d t^{*}}\right]}{\gamma\left(\boldsymbol{V}_{\mathrm{C}}\right)} \\
& =-\frac{\sum_{a}\left[\frac{m_{a} \cdot c^{2}}{\gamma_{a}{ }^{*}}+e_{a} \cdot \varphi^{*}\left(\boldsymbol{r}_{\boldsymbol{a}}^{*}, t^{*}\right)\right]}{\gamma\left(\boldsymbol{V}_{\mathrm{C}}\right)}+\sum_{a} \frac{e_{a}}{c} \boldsymbol{A}^{*}\left(\boldsymbol{r}_{\boldsymbol{a}}^{*}, t^{*}\right) \frac{d \boldsymbol{r}_{\boldsymbol{a}}^{*}}{d t}-\frac{\sum_{a} \frac{e_{a}}{c} \cdot\left(\frac{\partial f\left(\boldsymbol{r}_{\boldsymbol{a}}^{*}, t^{*}\right)}{\partial t^{*}}-\frac{\partial f\left(\boldsymbol{r}_{\boldsymbol{a}}^{*}, t^{*}\right)}{\partial \boldsymbol{r}_{\boldsymbol{a}}^{*}} \frac{d \boldsymbol{r}_{\boldsymbol{a}}^{*}}{d t^{*}}\right)}{\gamma\left(\boldsymbol{V}_{\mathrm{C}}\right)} \\
& =-\frac{\sum_{a}\left[\frac{m_{a} \cdot c^{2}}{\gamma_{a}{ }^{*}}+e_{a} \cdot \varphi^{*}\left(\boldsymbol{r}_{\boldsymbol{a}}^{*}, t^{*}\right)+\frac{e_{a}}{c} \frac{\partial f\left(\boldsymbol{r}_{\boldsymbol{a}}^{*}, t^{*}\right)}{\partial t^{*}}\right]}{\gamma\left(\boldsymbol{V}_{\mathbf{C}}\right)}+\sum_{a} \frac{e_{a}}{c} \boldsymbol{A}^{*}\left(\boldsymbol{r}_{\boldsymbol{a}}^{*}, t^{*}\right) \frac{d \boldsymbol{r}_{\boldsymbol{a}}^{*}}{d t}+\sum_{a} \frac{e_{a}}{c} \frac{\partial f\left(\boldsymbol{r}_{\boldsymbol{a}}^{*}, t^{*}\right)}{\partial \boldsymbol{r}_{\boldsymbol{a}}^{*}} \frac{d \boldsymbol{r}_{\boldsymbol{a}}^{*}}{d t}
\end{aligned}
$$

Thus 


$$
\begin{gathered}
L_{\text {modif }}^{\prime}\left(\left\{\boldsymbol{r}_{\boldsymbol{a}}^{*}(t)\right\},\left\{\frac{d \boldsymbol{r}_{a}^{*}}{d t}\right\}, \boldsymbol{R}_{\mathbf{C}}, \boldsymbol{V}_{\mathrm{C}}, t\right)=-\frac{\sum_{a}\left[\frac{m_{a} \cdot c^{2}}{\gamma_{a}^{*}}+e_{a} \cdot \varphi^{*}\left(\boldsymbol{r}_{\boldsymbol{a}}^{*}, t^{*}\right)+\frac{e_{a}}{c} \frac{\partial f\left(\boldsymbol{r}_{\boldsymbol{a}}^{*}, t^{*}\right)}{\partial t^{*}}\right]}{\gamma\left(\boldsymbol{V}_{\mathbf{C}}\right)}+\sum_{a} \frac{e_{a}}{c} \boldsymbol{A}^{*}\left(\boldsymbol{r}_{a}^{*}, t^{*}\right) \frac{d \boldsymbol{r}_{a}^{*}}{d t}+\sum_{a} \frac{e_{a} \partial f\left(\boldsymbol{r}_{\boldsymbol{a}}^{*}, t^{*}\right)}{\partial \boldsymbol{r}_{\boldsymbol{a}}^{*}} \frac{d \boldsymbol{r}_{a}^{*}}{d t} \\
=>\boldsymbol{P}_{\boldsymbol{c}}=\frac{\partial L_{\text {modif }}^{\prime}}{\partial \boldsymbol{V}_{\mathbf{C}}}=\gamma\left(\boldsymbol{V}_{\mathbf{C}}\right)\left(\frac{\sum_{a}\left[\gamma_{a}^{*} \cdot m_{a} \cdot c^{2}+e_{a} \cdot \varphi^{*}\left(\boldsymbol{r}_{\boldsymbol{a}}^{*}, t^{*}\right)\right]+\frac{e_{a}}{c} \frac{\partial f\left(\boldsymbol{r}_{\boldsymbol{a}}^{*}, t^{*}\right)}{\partial t^{*}}}{c^{2}}\right) \boldsymbol{V}_{\mathbf{C}}
\end{gathered}
$$

Which gives a mass:

$$
\begin{gathered}
M_{\text {modif }}=\frac{\sum_{a}\left[\gamma_{a}{ }^{*} \cdot m_{a} \cdot c^{2}+e_{a} \cdot \varphi_{\text {modif }}{ }^{*}\left(\boldsymbol{r}_{\boldsymbol{a}}^{*}, t^{*}\right)\right]}{c^{2}}=\frac{\sum_{a}\left[\gamma_{a}{ }^{*} \cdot m_{a} \cdot c^{2}+e_{a} \cdot \varphi^{*}\left(\boldsymbol{r}_{\boldsymbol{a}}^{*}, t^{*}\right)+\frac{e_{a}}{c} \frac{\partial f\left(\boldsymbol{r}_{\boldsymbol{a}}^{*}, t^{*}\right)}{\partial t^{*}}\right]}{c^{2}} \\
=M_{\text {free }}+M_{\text {interaction,modif }} \\
=M_{\text {free }}+M_{\text {interaction }}+M_{\text {gauge }}
\end{gathered}
$$

With $M_{\text {free }} \equiv \frac{\sum_{a} \gamma_{a}^{*} \cdot m_{a} \cdot c^{2}}{c^{2}} ; M_{\text {interaction }} \equiv \frac{\sum_{a} e_{a} \cdot \varphi^{*}\left(\boldsymbol{r}_{a}^{*}, t^{*}\right)}{c^{2}} ; M_{\text {gauge }} \equiv \frac{\sum_{a}\left[\frac{e_{a} \partial f\left(r_{\boldsymbol{r}}^{*}, t^{*}\right)}{\partial t^{*}}\right]}{c^{2}}$

In this case we cannot evacuate the mass $M_{\text {gauge }}$ by using the relativistic invariance argument since the additive gauge term $\frac{\partial f\left(x_{a j}^{*}\right)}{\partial x_{a i}^{*}} d x_{a i}^{*}$ is an existing relativistic invariant. Indeed, contrary to the case of the free material system (where we cannot express the additive term due to the non-existence of other relativistic expression than the one for the line element $\mathrm{ds}$ ) the addidive gauge term is not a material term but a field term whose expression or existence is guaranteed by the field equations of electromagnetism: $f\left(x_{a j}^{*}\right)$ is a dynamical variable, a scalar field, already an invariant that does not need to be expressed via other invariant material terms.

The arbitrariness of the value of the mass is here of course not a physical problem since the least action principle ensures us that changing the mass in this way does not change the equations dynamics, at least in a observable way (=seen from the material points).

However, we see that the mass is in general affected by the chosen gauge...but:

- This is only proven here for the specific case where the system considered is in an external field;

- The value of the mass is well define for a free (material) system thanks to the relativistic invariant requirement.

Moreover,

$\circ$ by repeating a king of the same proof of the paragraph 96 of [1] in the context of the Einstein gravitation theory, where Landau-Lifchitz shows us that the 4-momentum can be well defined despite the impact of the coordinate system modification (in the case where the system is "free" and surrounded by a Galilean region),

- we can say that for a material system locally in interaction with an electromagnetic field, if the whole system (particles and electromagnetic field) is surrounded by an "empty" region (with no mass, no 4-electromagnetic potential and so no gauge term as $f\left(x_{a j}^{*}\right)$ ) the 4- 
momentum is well defined and so also the mass. Indeed, the modification of the coordinate system in the Landau reasoning can be replaced a priori by the modification of the electromagnetic gauge.

- The origin of the Energy scale for material system in an external Electromagnetic field

By definition the energy is:

$$
\begin{aligned}
& E^{\prime} \equiv \sum_{a} \frac{\partial L^{\prime}}{\partial \frac{d r_{a}^{*}}{d t}} \frac{d \boldsymbol{r}_{a}^{*}}{d t}+\frac{\partial L^{\prime}}{\partial \boldsymbol{v}_{\mathrm{c}}} \boldsymbol{V}_{\boldsymbol{c}}-L^{\prime} \\
& E_{\text {modif }} \equiv \sum_{a} \frac{\partial L_{\text {modif }}{ }^{\prime}}{\partial \frac{d \boldsymbol{r}_{\boldsymbol{a}}^{*}}{d t}} \frac{d \boldsymbol{r}_{\boldsymbol{a}}^{*}}{d t}+\frac{\partial L_{\text {modif }^{\prime}}}{\partial \boldsymbol{V}_{\mathbf{C}}} \boldsymbol{V}_{\boldsymbol{c}}-L_{\text {modif }}{ }^{\prime}
\end{aligned}
$$

with

$$
\begin{aligned}
& L_{\text {modif }}^{\prime}\left(\left\{\boldsymbol{r}_{a}^{*}(t)\right\},\left\{\frac{d \boldsymbol{r}_{a}^{*}}{d t}\right\}, \boldsymbol{R}_{\mathrm{C}}, \boldsymbol{V}_{\mathrm{C}}, t\right)=-\frac{\sum_{a}\left[\frac{m_{a} \cdot c^{2}}{\gamma_{a}^{*}}+e_{a} \cdot \varphi^{*}\left(\boldsymbol{r}_{a}^{*}, t^{*}\right)+\frac{e_{a}}{c} \frac{\partial f\left(\boldsymbol{r}_{a}^{*}, t^{*}\right)}{\partial t^{*}}\right]}{\gamma\left(\boldsymbol{V}_{\mathrm{C}}\right)}+\sum_{a} \frac{e_{a}}{c} \frac{d r_{a}^{*}}{d t}+\sum_{a} \frac{e_{a}}{c} \frac{\partial f\left(\boldsymbol{r}_{a}^{*}, t^{*}\right)}{\partial \boldsymbol{r}_{a}^{*}} \frac{d \boldsymbol{r}_{a}^{*}}{d t} \\
& =L^{\prime}\left(\left\{\boldsymbol{r}_{a}^{*}(t)\right\},\left\{\frac{d \boldsymbol{r}_{a}^{*}}{d t}\right\}, \boldsymbol{R}_{\mathrm{C}}, \boldsymbol{V}_{\mathrm{C}}, t\right)-\frac{\sum_{a}\left[\frac{e_{a}}{c} \frac{\partial f\left(\boldsymbol{r}_{a}^{*}, t^{*}\right)}{\partial t^{*}}\right]}{\gamma\left(\boldsymbol{V}_{\mathrm{C}}\right)}+\sum_{a} \frac{e_{a}}{c} \frac{\partial f\left(\boldsymbol{r}_{a}^{*}, t^{*}\right)}{\partial \boldsymbol{r}_{a}^{*}} \frac{d \boldsymbol{r}_{a}^{*}}{d t} \\
& E_{\text {modif }} \equiv \sum_{a} \frac{\partial\left(L^{\prime}\left(\left\{\boldsymbol{r}_{\boldsymbol{a}}^{*}(t)\right\},\left\{\frac{d \boldsymbol{r}_{a}^{*}}{d t}\right\}, \boldsymbol{R}_{\mathrm{C}}, \boldsymbol{V}_{\mathrm{C}}, t\right)-\frac{\sum_{a}\left[\frac{e_{a}}{c} \frac{\partial f\left(\boldsymbol{r}_{a}^{*}, t^{*}\right)}{\partial t^{*}}\right]}{\gamma\left(\boldsymbol{V}_{\mathrm{C}}\right)}+\sum_{a} \frac{e_{a}}{c} \frac{\partial f\left(\boldsymbol{r}_{a}^{*}, t^{*}\right)}{\partial \boldsymbol{r}_{a}^{*}} \frac{d \boldsymbol{r}_{a}^{*}}{d t}\right)}{\partial \frac{d \boldsymbol{r}_{a}^{*}}{d t}} \frac{d \boldsymbol{r}_{a}^{*}}{d t} \\
& +\left(\gamma\left(\boldsymbol{V}_{\mathrm{c}}\right)\left(\frac{\sum_{a}\left[\gamma_{a}^{*} \cdot m_{a} \cdot c^{2}+e_{a} \cdot \varphi^{*}\left(\boldsymbol{r}_{a}^{*}, t^{*}\right)\right]+\frac{e_{a}}{c} \frac{\partial f\left(\boldsymbol{r}_{a}^{*}, t^{*}\right)}{\partial t^{*}}}{c^{2}}\right) \boldsymbol{V}_{\mathbf{c}}\right) \boldsymbol{V}_{\boldsymbol{c}} \\
& -\left(L^{\prime}\left(\left\{\boldsymbol{r}_{\boldsymbol{a}}^{*}(t)\right\},\left\{\frac{d \boldsymbol{r}_{a}^{*}}{d t}\right\}, \boldsymbol{R}_{\mathrm{C}}, \boldsymbol{V}_{\mathrm{C}}, t\right)-\frac{\sum_{a}\left[\frac{e_{a}}{c} \frac{\partial f\left(\boldsymbol{r}_{a}^{*}, t^{*}\right)}{\partial t^{*}}\right]}{\gamma\left(\boldsymbol{V}_{\mathrm{C}}\right)}+\sum_{a} \frac{e_{a}}{c} \frac{\partial f\left(\boldsymbol{r}_{a}^{*}, t^{*}\right)}{\partial \boldsymbol{r}_{a}^{*}} \frac{d \boldsymbol{r}_{a}^{*}}{d t}\right)
\end{aligned}
$$

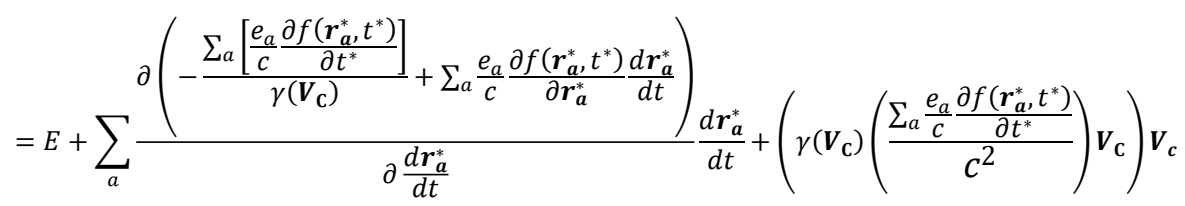

$$
\begin{aligned}
& -\left(-\frac{\sum_{a}\left[\frac{e_{a}}{c} \frac{\partial f\left(\boldsymbol{r}_{a}^{*}, t^{*}\right)}{\partial t^{*}}\right]}{\gamma\left(\boldsymbol{V}_{\mathrm{C}}\right)}+\sum_{a} \frac{e_{a}}{c} \frac{\partial f\left(\boldsymbol{r}_{a}^{*}, t^{*}\right)}{\partial \boldsymbol{r}_{a}^{*}} \frac{d \boldsymbol{r}_{a}^{*}}{d t}\right) \\
& =E+\sum_{a} \frac{e_{a}}{c} \frac{\partial f\left(\boldsymbol{r}_{a}^{*}, t^{*}\right)}{\partial \boldsymbol{r}_{a}^{*}} \frac{d \boldsymbol{r}_{a}^{*}}{d t}+\left(\gamma\left(\boldsymbol{V}_{\mathrm{C}}\right)\left(\frac{\sum_{a} \frac{e_{a}}{c} \frac{\partial f\left(\boldsymbol{r}_{a}^{*}, t^{*}\right)}{\partial t^{*}}}{c^{2}}\right) \boldsymbol{V}_{\mathbf{C}}\right) \boldsymbol{V}_{c}-\left(-\frac{\sum_{a}\left[\frac{e_{a}}{c} \frac{\partial f\left(\boldsymbol{r}_{a}^{*}, t^{*}\right)}{\partial t^{*}}\right]}{\gamma\left(\boldsymbol{V}_{\mathbf{C}}\right)}+\sum_{a} \frac{e_{a}}{c} \frac{\partial f\left(\boldsymbol{r}_{a}^{*}, t^{*}\right)}{\partial \boldsymbol{r}_{a}^{*}} \frac{d \boldsymbol{r}_{a}^{*}}{d t}\right) \\
& =E+\left(\sum_{a} \frac{e_{a}}{c} \frac{\partial f\left(\boldsymbol{r}_{a}^{*}, t^{*}\right)}{\partial t^{*}}\right)\left[\gamma\left(\boldsymbol{V}_{\mathbf{C}}\right) \beta^{2}+\frac{1}{\gamma\left(\boldsymbol{V}_{\mathbf{C}}\right)}\right]=E+\left(\sum_{a} \frac{e_{a}}{c} \frac{\partial f\left(\boldsymbol{r}_{a}^{*}, t^{*}\right)}{\partial t^{*}}\right)\left[\frac{\gamma\left(\boldsymbol{V}_{\mathbf{C}}\right)^{2} \beta^{2}+1}{\gamma\left(\boldsymbol{V}_{\mathbf{C}}\right)}\right]=E+\left(\sum_{a} \frac{e_{a}}{c} \frac{\partial f\left(\boldsymbol{r}_{a}^{*}, t^{*}\right)}{\partial t^{*}}\right) \gamma \\
& =\gamma\left(E^{*}+\sum_{a} \frac{e_{a}}{c} \frac{\partial f\left(r_{a}^{*}, t^{*}\right)}{\partial t^{*}}\right) \\
& =>E_{\text {modi }}=\gamma\left(E_{\text {free }}{ }^{*}+E_{\text {interaction }}{ }^{*}+\sum_{a} \frac{e_{a}}{c} \frac{\partial f\left(r_{a^{*}}^{*} t^{*}\right)}{\partial t^{*}}\right)
\end{aligned}
$$


But we know that

$$
M_{\text {modif }}=M_{\text {free }}+M_{\text {interaction }}+\frac{\sum_{a}\left[\frac{e_{a}}{c} \frac{\partial f\left(\boldsymbol{r}_{a}^{*}, t^{*}\right)}{\partial t^{*}}\right]}{c^{2}}
$$

Thus we can also write

$$
E_{\text {modif }}=\gamma M_{\text {modif }} c^{2}
$$

As above, the value of the mass sets the origin of the Energy scale even if:

o The system is in an external field;

$\circ$ And the gauge was modified.

We are again in accordance with Landau-Lifchitz [1].

If one decides to define the mass only for free system we have:

$$
E_{\text {modif }}=\gamma M c^{2}+\gamma\left(E_{\text {interaction }}{ }^{*}+\sum_{a} \frac{e_{a}}{c} \frac{\partial f\left(r_{a^{*}}^{*} t^{*}\right)}{\partial t^{*}}\right)
$$

In this case the origin of the Energy scale is:

$$
E_{\text {modif }, 0}=M c^{2}+E_{\text {interaction }}^{*}+\sum_{a} \frac{e_{a}}{c} \frac{\partial f\left(\boldsymbol{r}_{\boldsymbol{a}}^{*}, t^{*}\right)}{\partial t^{*}}
$$

We see that with this kind of definition of the mass, the origin of the Energy scale $E_{\text {modif }, 0}$ is no more the mass. So:

- Either :

- we set the origin of the Energy scale by the mass

$$
E_{\text {modif }, 0}=M c^{2}
$$

○ but we work with a mass dependent of the gauge (the most "natural" case):

$$
M=M_{\text {free }}+M_{\text {interaction }}+M_{\text {gauge }}
$$

- Either:

- we don't set the origin of the Energy scale by the mass:

$$
E_{\text {modif }, 0}=M c^{2}+E_{\text {interaction }}{ }^{*}+E_{\text {gauge }}
$$

O in order to keep a fixed value for the mass (the most arbitrary case but "stable"):

$$
M=M_{\text {free }}
$$




\subsection{A material system in a non-Minkowskian space-time (General Relativity)}

\section{- The momentum \& the mass for material system in a non-Minkowskian space-time}

The variability of the mass value appears also if we take account General Relativity. But, contrary of the Electromagnetism \& al, the gravitational field is always present. Indeed there is no sense to talk about space time without gravitation since we cannot put the metric equal to zero (even in Minkowsky space-time which is a particular gravitational field). This point is for me the fundamental idea of General Relativity, without that, any (directly) unobservable frame of reference would be considered as a possible cause for the acceleration of a body [22]: it would be a come-back to the "ugly" pre-Machian (as understood by Einstein) privileged frame of reference...

So in general the mass of a material system in General Relativity always depends on the gravitational field, as stated by Einstein in his first article on cosmology in 1917 [8]. Fundamentally, there is only a very particular case where we can define the mass in a systematic way in GR (independently to the context): the case where the system is infinitesimally small...since the Equivalence principle certifies us that the gravitation field is always sufficiently smooth to be sure to encounter in any infinitesimal space-time a quasi-Minskowskian space-time. However, in practice, we can also have a finite volume Minkowskian space-time ("Galilean domain") where we can fix the mass. But again, it is fundamentally accidental (=not at all necessary) in the spirit of GR.

$$
\begin{gathered}
S\left[\left\{\boldsymbol{r}_{\boldsymbol{a}}(t)\right\}, t\right]=\int_{s_{1}}^{s_{2}} \sum_{a}\left[-m_{a} \cdot c d s_{a}\right]=\int_{t_{1}}^{t_{2}} \sum_{a}\left[-m_{a} \cdot c \sqrt{g_{i k}\left(x_{a}^{j}\right) d x_{a}^{i} d x_{a}^{k}}\right] \\
=\int_{t_{1}}^{t_{2}} \sum_{a}\left[-m_{a} \cdot c \sqrt{\left.g_{i k}\left(x_{a}^{j}\right) \frac{d x_{a}^{i}}{d t} \frac{d x_{a}^{k}}{d t}\right] d t}\right. \\
=>L\left(\left\{\boldsymbol{r}_{\boldsymbol{a}}(t)\right\},\left\{\frac{d \boldsymbol{r}_{\boldsymbol{a}}^{*}}{d t}\right\}, t\right)=\sum_{a}\left[-m_{a} \cdot c \sqrt{g_{i k}\left(x_{a}^{j}\right) \frac{d x_{a}^{i}}{d t} \frac{d x_{a}^{k}}{d t}}\right]
\end{gathered}
$$

Like above, we have also:

$$
\begin{aligned}
& S\left[\left\{\boldsymbol{r}_{\boldsymbol{a}}^{*}(t)\right\}, \boldsymbol{R}_{\mathbf{C}}(t)\right]=\int_{t_{1}}^{t_{1}}\left(\frac{\sum_{a}\left[-m_{a} \cdot c \sqrt{g_{i k}^{*}\left(x_{a}^{j *}\right) \frac{d x_{a}^{i *}}{d t^{*}} \frac{d x_{a}^{k *}}{d t^{*}}}\right]}{\gamma\left(\boldsymbol{V}_{\mathbf{C}}\right)}\right) d t \\
= & L^{\prime}\left(\left\{\boldsymbol{r}_{\boldsymbol{a}}^{*}(t)\right\},\left\{\frac{d \boldsymbol{r}_{\boldsymbol{a}}^{*}}{d t}\right\}, \boldsymbol{R}_{\mathbf{C}}, \boldsymbol{V}_{\mathbf{C}}, t\right)=\frac{\sum_{a}\left[-m_{a} \cdot c \sqrt{g_{i k^{*}}\left(x_{a}^{j *}\right) \frac{d x_{a}^{i *}}{d t^{*}} \frac{d x_{a}^{k *}}{d t^{*}}}\right]}{\gamma\left(\boldsymbol{V}_{\mathbf{C}}\right)}
\end{aligned}
$$

If we develop the expression 


$$
\begin{aligned}
& L^{\prime}\left(\left\{\boldsymbol{r}_{\boldsymbol{a}}^{*}(t)\right\},\left\{\frac{d \boldsymbol{r}_{\boldsymbol{a}}^{*}}{d t}\right\}, \boldsymbol{R}_{\mathrm{C}}, \boldsymbol{V}_{\mathrm{C}}, t\right) \\
& =\frac{\sum_{a}\left[-m_{a} \cdot c \sqrt{\left.g_{00}{ }^{*}\left(\boldsymbol{r}_{\boldsymbol{a}}^{*}, t^{*}\right) c^{2}+2 g_{0 \alpha^{*}}\left(\boldsymbol{r}_{\boldsymbol{a}}^{*}, t^{*}\right) \frac{d x_{a}^{\alpha *}}{d t^{*}} c+g_{\alpha \beta}{ }^{*}\left(\boldsymbol{r}_{\boldsymbol{a}}^{*}, t^{*}\right) \frac{d x_{a}^{\alpha *}}{d t^{*}} \frac{d x_{a}^{\beta *}}{d t^{*}}\right]}\right.}{\gamma\left(\boldsymbol{V}_{\mathbf{c}}\right)} \\
& =\frac{\sum_{a}\left[-m_{a} \cdot c \sqrt{\left.g_{00}{ }^{*}\left(\boldsymbol{r}_{\boldsymbol{a}}^{*}, t^{*}\right) c^{2}+\gamma\left(\boldsymbol{V}_{\mathbf{C}}\right) 2 g_{0 \alpha^{*}}\left(\boldsymbol{r}_{\boldsymbol{a}}^{*}, t^{*}\right) \frac{d x_{a}^{\alpha *}}{d t} c+\gamma\left(\boldsymbol{V}_{\mathbf{C}}\right)^{2} g_{\alpha \beta}{ }^{*}\left(\boldsymbol{r}_{\boldsymbol{a}}^{*}, t^{*}\right) \frac{d x_{a}^{\alpha *}}{d t} \frac{d x_{a}^{\beta *}}{d t}\right]}\right.}{\gamma\left(\boldsymbol{V}_{\mathbf{C}}\right)} \\
& =\frac{\sum_{a}\left[-m_{a} \cdot c \sqrt{\left.g_{00}{ }^{*}\left(\boldsymbol{r}_{\boldsymbol{a}}^{*}, t^{*}\right) c^{2}+\gamma\left(\boldsymbol{V}_{\mathbf{C}}\right) 2 g_{0 \alpha^{*}}{ }^{*}\left(\boldsymbol{r}_{\boldsymbol{a}}^{*}, t^{*}\right) \frac{d x_{a}^{\alpha *} c}{d t}+\gamma\left(\boldsymbol{V}_{\mathbf{C}}\right)^{2} g_{\alpha \beta}{ }^{*}\left(\boldsymbol{r}_{\boldsymbol{a}}^{*}, t^{*}\right) \frac{d x_{a}^{\alpha *}}{d t} \frac{d x_{a}^{\beta *}}{d t}\right]}\right.}{\gamma\left(\boldsymbol{V}_{\mathbf{C}}\right)}
\end{aligned}
$$

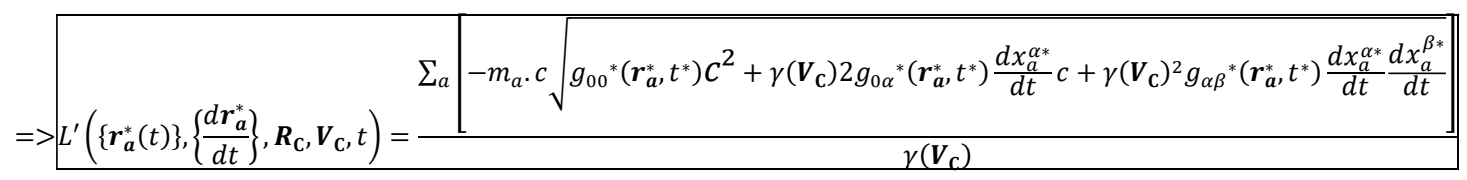

$$
\begin{aligned}
& \boldsymbol{P}_{\boldsymbol{c}}=\frac{\partial L^{\prime}}{\partial \boldsymbol{V}_{\mathbf{C}}}=\sum_{a}\left[-m_{a} \cdot c \frac{\partial}{\partial \boldsymbol{V}_{\mathbf{C}}} \sqrt{\left.\frac{g_{00}{ }^{*}\left(r_{a}^{*}, t^{*}\right)}{\gamma\left(\boldsymbol{V}_{\mathbf{C}}\right)^{2}} c^{2}+\frac{1}{\gamma\left(\boldsymbol{V}_{\mathrm{C}}\right)} 2 g_{0 \alpha}{ }^{*}\left(\boldsymbol{r}_{a}^{*}, t^{*}\right) \frac{d x_{a}^{\alpha *}}{d t} c+g_{\alpha \beta}{ }^{*}\left(\boldsymbol{r}_{a}^{*}, t^{*}\right) \frac{d x_{a}^{\alpha^{* *}}}{d t} \frac{d x_{a}^{\beta^{*}}}{d t}\right]}\right. \\
& =\sum_{a}\left[-m_{a} \cdot c \frac{1}{2} \frac{\left(g_{00}{ }^{*}\left(r_{a}^{*}, t^{*}\right) c^{2} \frac{\partial}{\partial \boldsymbol{V}_{\mathrm{C}}} \frac{1}{\gamma\left(\boldsymbol{V}_{\mathrm{C}}\right)^{2}}+2 g_{0 \alpha}{ }^{*}\left(\boldsymbol{r}_{a}^{*}, t^{*}\right) \frac{d x_{a}^{\alpha *}}{d t} c \frac{\partial}{\partial \boldsymbol{V}_{\mathrm{C}}} \frac{1}{\gamma\left(\boldsymbol{V}_{\mathrm{C}}\right)}\right)}{\sqrt{\frac{g_{00}{ }^{*}\left(r_{a}^{*}, t^{*}\right)}{\gamma\left(\boldsymbol{V}_{\mathrm{C}}\right)^{2}} c^{2}+\frac{1}{\gamma\left(\boldsymbol{V}_{\mathrm{C}}\right)} 2 g_{0 \alpha}{ }^{*}\left(\boldsymbol{r}_{a}^{*}, t^{*}\right) \frac{d x_{a}^{\alpha *}}{d t} c+g_{\alpha \beta}{ }^{*}\left(\boldsymbol{r}_{a}^{*}, t^{*}\right) \frac{d x_{a}^{\alpha *}}{d t} \frac{d x_{a}^{\beta *}}{d t}}}\right]
\end{aligned}
$$

- $\frac{\partial}{\partial V_{\mathrm{c}}} \frac{1}{\gamma\left(V_{\mathrm{c}}\right)}=\frac{\partial}{\partial v_{\mathrm{c}}} \sqrt{1-\frac{V_{\mathrm{c}}^{2}}{c^{2}}}=\frac{-\frac{1}{2} 2 \frac{v_{\mathrm{C}}}{c^{2}}}{\sqrt{1-\frac{V_{\mathrm{c}}^{2}}{c^{2}}}}=-\gamma\left(V_{\mathrm{C}}\right) \frac{V_{\mathrm{c}}}{c^{2}}$

- $\frac{\partial}{\partial V_{\mathrm{c}}} \frac{1}{\gamma\left(V_{\mathrm{C}}\right)^{2}}=\frac{\partial\left(1-\frac{V_{\mathrm{C}}{ }^{2}}{c^{2}}\right)}{\partial v_{\mathrm{C}}}=-2 \frac{V_{\mathrm{c}}}{c^{2}}$

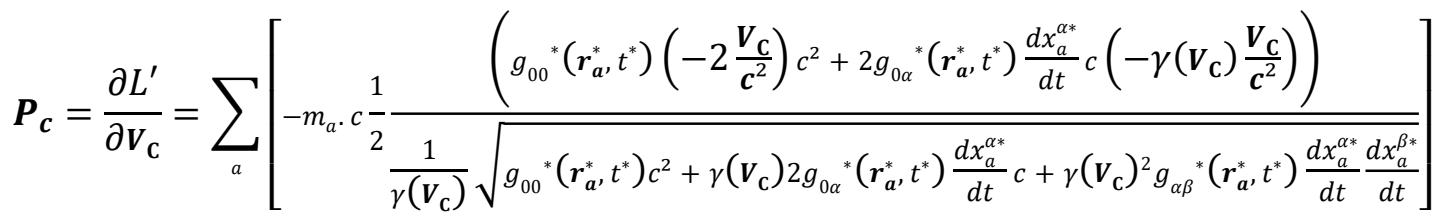

$$
\begin{aligned}
& =\frac{\boldsymbol{V}_{\mathbf{C}}}{c^{2}} \gamma\left(\boldsymbol{V}_{\mathrm{C}}\right) \sum_{a}\left[m_{a} \cdot c \frac{g_{00}{ }^{*}\left(\boldsymbol{r}_{a}^{*}, t^{*}\right) c^{2}+g_{0 \alpha}{ }^{*}\left(\boldsymbol{r}_{a}^{*}, t^{*}\right) \frac{d x_{a}^{\alpha *}}{d t} \gamma\left(\boldsymbol{V}_{\mathbf{C}}\right) c}{\sqrt{g_{00}{ }^{*}\left(\boldsymbol{r}_{a}^{*}, t^{*}\right) c^{2}+\gamma\left(\boldsymbol{V}_{\mathrm{C}}\right) 2 g_{0 \alpha}{ }^{*}\left(\boldsymbol{r}_{a}^{*}, t^{*}\right) \frac{d x_{a}^{\alpha *}}{d t} c+\gamma\left(\boldsymbol{V}_{\mathrm{C}}\right)^{2} g_{\alpha \beta}{ }^{*}\left(\boldsymbol{r}_{a}^{*}, t^{*}\right) \frac{d x_{a}^{\alpha *}}{d t} \frac{d x_{a}^{\beta *}}{d t}}}\right]
\end{aligned}
$$




$$
\begin{aligned}
& =\frac{\boldsymbol{V}_{\mathbf{C}}}{c^{2}} \gamma\left(\boldsymbol{V}_{\mathbf{C}}\right) \sum_{a}\left[m_{a} \cdot c \frac{g_{00}{ }^{*}\left(\boldsymbol{r}_{a^{*}}, t^{*}\right) c^{2}+g_{0 \alpha}{ }^{*}\left(\boldsymbol{r}_{\boldsymbol{a}^{*}}, t^{*}\right) \frac{d x_{a}^{\alpha *}}{d t} \gamma\left(\boldsymbol{V}_{\mathbf{C}}\right) c}{\frac{d s}{d t^{*}}}\right] \\
& =\frac{\boldsymbol{V}_{\mathbf{C}}}{c^{2}} \gamma\left(\boldsymbol{V}_{\mathbf{C}}\right) \sum_{a}\left[m_{a} \cdot c^{2} \frac{g_{00}{ }^{*}\left(\boldsymbol{r}_{a^{*}}^{*}, t^{*}\right) c \frac{d t^{*}}{d t^{*}}+g_{0 \alpha}{ }^{*}\left(\boldsymbol{r}_{a}^{*}, t^{*}\right) \frac{d x_{a}^{\alpha *}}{d t^{*}}}{\frac{d s}{d t^{*}}}\right] \\
& =\frac{\boldsymbol{V}_{\mathbf{C}}}{c^{2}} \gamma\left(\boldsymbol{V}_{\mathbf{C}}\right) \sum_{a}\left[m_{a} \cdot c^{2} \frac{g_{0 i}{ }^{*}\left(\boldsymbol{r}_{a}^{*}, t^{*}\right) \frac{d i_{a}^{i *}}{d t^{*}}}{\frac{d s}{d t^{*}}}\right]=\frac{\boldsymbol{V}_{\mathbf{C}}}{c^{2}} \gamma\left(\boldsymbol{V}_{\mathbf{C}}\right) \sum_{a}\left[m_{a} \cdot c^{2} g_{0 i}{ }^{*}\left(\boldsymbol{r}_{a}^{*}, t^{*}\right) \frac{d x_{a}^{i *}}{d s}\right] \\
& =\frac{\boldsymbol{V}_{\mathbf{C}}}{c^{2}} \gamma\left(\boldsymbol{V}_{\mathrm{C}}\right) \sum_{a} m_{a} \cdot c^{2} g_{0 i}{ }^{*}\left(\boldsymbol{r}_{a}^{*}, t^{*}\right) u_{a}^{i *} \\
& \boldsymbol{P}_{\boldsymbol{c}}=\frac{\partial L^{\prime}}{\partial \boldsymbol{V}_{\mathbf{C}}}=\gamma\left(\boldsymbol{V}_{\mathbf{C}}\right) \frac{E^{*}}{c^{2}} \boldsymbol{V}_{\mathbf{C}} \\
& \text { With } \\
& E^{*}=\sum_{a} E_{a}^{*}=\sum_{a} m_{a} \cdot c^{2} g_{0 i}{ }^{*}\left(\boldsymbol{r}_{\boldsymbol{a}}^{*}, t^{*}\right) u_{a}^{i *}
\end{aligned}
$$

According to Landau-Lifchitz in [1] in the paragraph 88:

○ if we take correctly account the a priori de-synchronization between the temporal coordinates of different points of a coordinate system in a stationary metric field in $\mathrm{K}^{*}$,

○ the expression $E_{a}^{*}=m_{a} \cdot c^{2} g_{0 i}{ }^{*}\left(\boldsymbol{r}_{\boldsymbol{a}}^{*}, t^{*}\right) u_{a}^{i *}$ can be also express also

$$
E_{a}^{*}=m_{a} \cdot c^{2} g_{0 i}{ }^{*}\left(\boldsymbol{r}_{a}^{*}, t^{*}\right) u_{a}^{i *}=\left(\frac{m_{a} \cdot c^{2} \sqrt{g_{00}{ }^{*}\left(\boldsymbol{r}_{a}^{*}\right)}}{\sqrt{1-\frac{1}{c^{2}}\left(\frac{d \boldsymbol{r}_{a}^{*}}{d \tau^{*}}\right)^{2}}}\right)_{d \tau^{*}=\frac{\sqrt{g_{00^{*}}}}{c}\left(d x_{0}{ }^{*}+\frac{g_{0 \alpha^{*}} * \alpha^{*}}{g_{00}} d x^{*}\right)}
$$

Which gives a mass:

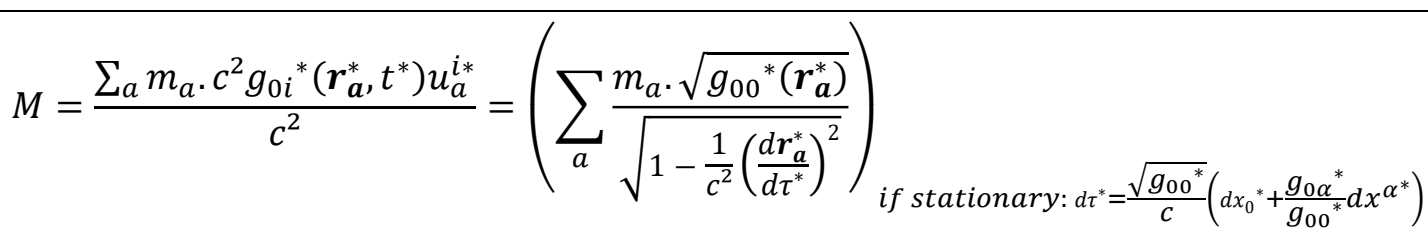

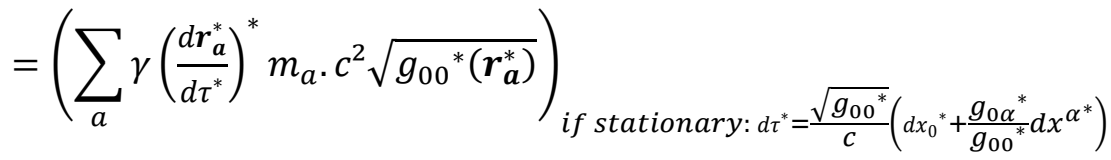




\section{- The origin of the Energy scale for material system in non-Minkowskian space-time}

By definition the energy is:

$$
E \equiv \sum_{a} \frac{\partial L^{\prime}}{\partial \frac{d \boldsymbol{r}_{\boldsymbol{a}}^{*}}{d t}} \frac{d \boldsymbol{r}_{a}^{*}}{d t}+\frac{\partial L^{\prime}}{\partial \boldsymbol{V}_{\mathbf{c}}} \boldsymbol{V}_{\boldsymbol{c}}-L^{\prime}
$$

$L^{\prime}\left(\left\{\boldsymbol{r}_{\boldsymbol{a}}^{*}(t)\right\},\left\{\frac{d \boldsymbol{r}_{\boldsymbol{a}}^{*}}{d t}\right\}, \boldsymbol{R}_{\mathbf{C}}, \boldsymbol{V}_{\mathbf{C}}, t\right)=\frac{\sum_{a}\left[-m_{a} \cdot c \sqrt{g_{i k}{ }^{*}\left(x_{a}^{j *}\right) \frac{d x_{a}^{i *}}{d t^{*}} \frac{d x_{a}^{k *}}{d t^{*}}}\right]}{\gamma\left(\boldsymbol{V}_{\mathbf{C}}\right)}$

$$
=\frac{\sum_{a}\left[-m_{a} \cdot c \sqrt{\left.g_{00}{ }^{*}\left(\boldsymbol{r}_{a}^{*}, t^{*}\right) c^{2}+\gamma\left(\boldsymbol{V}_{\mathrm{C}}\right) 2 g_{0 \alpha}{ }^{*}\left(\boldsymbol{r}_{a}^{*}, t^{*}\right) \frac{d x_{a}^{\alpha *}}{d t} c+\gamma\left(\boldsymbol{V}_{\mathrm{C}}\right)^{2} g_{\alpha \beta}{ }^{*}\left(\boldsymbol{r}_{a}^{*}, t^{*}\right) \frac{d x_{a}^{\alpha *}}{d t} \frac{d x_{a}^{\beta *}}{d t}\right]}\right.}{\gamma\left(\boldsymbol{V}_{\mathrm{C}}\right)}
$$

$$
E \equiv \sum_{a} \frac{\partial\left(\frac{\sum a\left[-m_{a} \cdot c \sqrt{\left.g_{i k}{ }^{*}\left(x_{a}^{j *}\right) \frac{d x_{a}^{i *} d x^{* *}}{d t^{*}}\right]}\right.}{\gamma\left(\boldsymbol{V}_{\mathbf{C}}\right)}\right)}{\partial \frac{d r_{a}^{*}}{d t}} \frac{d \boldsymbol{r}_{a}^{*}}{d t}+\left(\gamma\left(\boldsymbol{V}_{\mathbf{C}}\right) \frac{E^{*}}{c^{2}} \boldsymbol{V}_{\mathbf{C}}\right) \boldsymbol{V}_{\boldsymbol{c}}-\left(\frac{\sum\left[-m_{a} \cdot c \sqrt{\left.g_{i k}^{*}\left(x_{a}^{j *}\right) \frac{d x_{a}^{i *} \frac{d x_{a}^{k *}}{d t^{*}}}{d t^{*}}\right]}\right.}{\gamma\left(\boldsymbol{V}_{\mathbf{C}}\right)}\right)
$$

with

$$
\frac{\partial\left(\frac{\left[-m_{a} \cdot c \sqrt{\left.g_{i k}^{*}\left(x_{a}^{j *}\right) \frac{d x_{a}^{i *}}{d t^{*}} \frac{d x_{a}^{k *}}{d t^{*}}\right]}\right.}{\gamma\left(\boldsymbol{V}_{\mathbf{C}}\right)}\right)}{\partial \frac{d r_{a}^{*}}{d t}}
$$

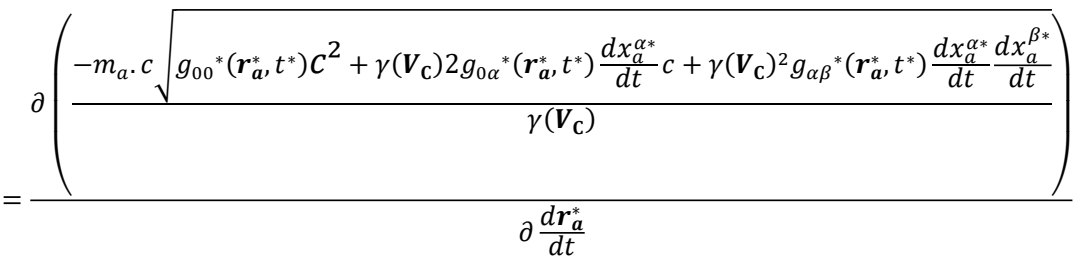

$=\frac{-m_{a} \cdot c}{\gamma\left(\boldsymbol{V}_{\mathbf{C}}\right)} \frac{\partial\left(\sqrt{g_{00}{ }^{*}\left(\boldsymbol{r}_{\boldsymbol{a}}^{*}, t^{*}\right) C^{2}+\gamma\left(\boldsymbol{V}_{\mathbf{C}}\right) 2 g_{0 \alpha}{ }^{*}\left(\boldsymbol{r}_{\boldsymbol{a}}^{*}, t^{*}\right) \frac{d x_{a}^{\alpha *}}{d t} c+\gamma\left(\boldsymbol{V}_{\mathbf{C}}\right)^{2} g_{\alpha \beta}{ }^{*}\left(\boldsymbol{r}_{\boldsymbol{a}}^{*}, t^{*}\right) \frac{d x_{a}^{\alpha *}}{d t} \frac{d x_{a}^{\beta *}}{d t}}\right)}{\partial \frac{d \boldsymbol{r}_{\boldsymbol{a}}^{*}}{d t}}$

with

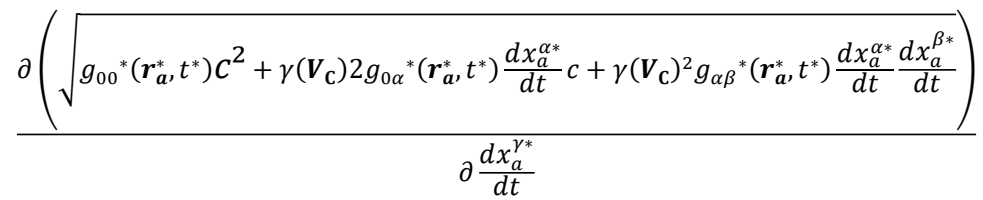

$=\frac{1}{2} \frac{\gamma\left(\boldsymbol{V}_{\mathbf{C}}\right) 2 g_{0 \alpha}{ }^{*}\left(\boldsymbol{r}_{\boldsymbol{a}}^{*}, t^{*}\right) \delta_{\alpha \gamma} c+2 \gamma\left(\boldsymbol{V}_{\mathbf{C}}\right)^{2} g_{\alpha \beta}{ }^{*}\left(\boldsymbol{r}_{\boldsymbol{a}}^{*}, t^{*}\right) \delta_{\alpha \gamma} \frac{d x_{a}^{\beta *}}{d t}}{\sqrt{g_{00}{ }^{*}\left(\boldsymbol{r}_{\boldsymbol{a}}^{*}, t^{*}\right) \boldsymbol{C}^{2}+\gamma\left(\boldsymbol{V}_{\mathbf{C}}\right) 2 g_{0 \alpha}{ }^{*}\left(\boldsymbol{r}_{\boldsymbol{a}}^{*}, t^{*}\right) \frac{d x_{a}^{\alpha *}}{d t} c+\gamma\left(\boldsymbol{V}_{\mathbf{C}}\right)^{2} g_{\alpha \beta}{ }^{*}\left(\boldsymbol{r}_{\boldsymbol{a}}^{*}, t^{*}\right) \frac{d x_{a}^{\alpha *}}{d t} \frac{d x_{a}^{\beta *}}{d t}}}$ 
$=\frac{\gamma\left(\boldsymbol{V}_{\mathbf{C}}\right) g_{0 \gamma}{ }^{*}\left(\boldsymbol{r}_{\boldsymbol{a}}^{*}, t^{*}\right) c+\gamma\left(\boldsymbol{V}_{\mathbf{C}}\right)^{2} g_{\gamma \beta}{ }^{*}\left(\boldsymbol{r}_{\boldsymbol{a}}^{*}, t^{*}\right) \frac{d x_{a}^{\beta *}}{d t}}{\frac{d s}{d t^{*}}}$

Then

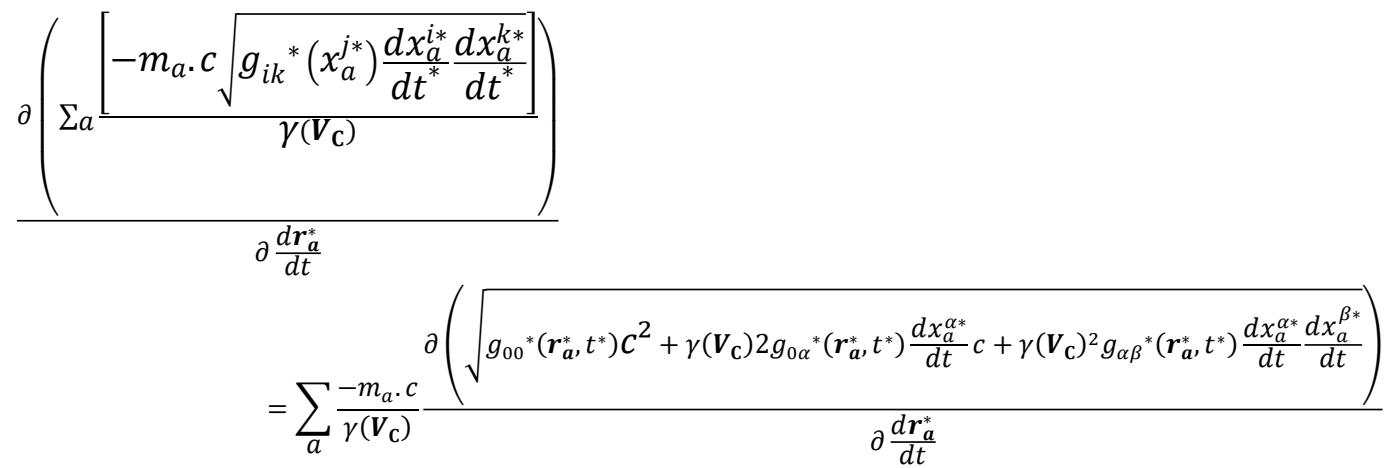

$=\sum_{a} \frac{-m_{a} \cdot c}{\gamma\left(\boldsymbol{V}_{\mathbf{C}}\right) \frac{d s}{d t^{*}}}\left(\begin{array}{l}\gamma\left(\boldsymbol{V}_{\mathbf{C}}\right) g_{01}{ }^{*}\left(\boldsymbol{r}_{\boldsymbol{a}}^{*}, t^{*}\right) c+\gamma\left(\boldsymbol{V}_{\mathbf{C}}\right)^{2} g_{1 \beta}{ }^{*}\left(\boldsymbol{r}_{\boldsymbol{a}}^{*}, t^{*}\right) \frac{d x_{a}^{\beta *}}{d t} \\ \gamma\left(\boldsymbol{V}_{\mathbf{C}}\right) g_{02}{ }^{*}\left(\boldsymbol{r}_{\boldsymbol{a}}^{*}, t^{*}\right) c+\gamma\left(\boldsymbol{V}_{\mathbf{C}}\right)^{2} g_{2 \beta^{*}}{ }^{*}\left(\boldsymbol{r}_{\boldsymbol{a}}^{*}, t^{*}\right) \frac{d x_{a}^{\beta *}}{d t} \\ \gamma\left(\boldsymbol{V}_{\mathbf{C}}\right) g_{03}{ }^{*}\left(\boldsymbol{r}_{\boldsymbol{a}}^{*}, t^{*}\right) c+\gamma\left(\boldsymbol{V}_{\mathbf{C}}\right)^{2} g_{3 \beta^{*}}{ }^{*}\left(\boldsymbol{r}_{\boldsymbol{a}}^{*}, t^{*}\right) \frac{d x_{a}^{\beta *}}{d t}\end{array}\right)$

Then

$$
\begin{gathered}
E=\sum_{a} \frac{-m_{a} \cdot c}{\gamma\left(\boldsymbol{V}_{\mathbf{C}}\right) \frac{d s}{d t^{*}}}\left(\gamma\left(\boldsymbol{V}_{\mathbf{C}}\right) g_{0 \alpha}{ }^{*}\left(\boldsymbol{r}_{a}^{*}, t^{*}\right) \frac{d x_{a}^{\alpha *}}{d t} c+\gamma\left(\boldsymbol{V}_{\mathbf{C}}\right)^{2} g_{\alpha \beta}{ }^{*}\left(\boldsymbol{r}_{a}^{*}, t^{*}\right) \frac{d x_{a}^{\beta *}}{d t} \frac{d x_{a}^{\alpha *}}{d t}\right)+\left(\gamma\left(\boldsymbol{V}_{\mathbf{C}}\right) \frac{E^{*}}{c^{2}} \boldsymbol{V}_{\mathbf{C}}\right) \boldsymbol{V}_{\boldsymbol{c}} \\
-\left(\frac{\sum_{a}\left[-m_{a} \cdot c \sqrt{g_{i k^{*}}\left(x_{a}^{j *}\right) \frac{d x_{a}^{i *}}{d t^{*}} \frac{d x_{a}^{k *}}{d t^{*}}}\right]}{\gamma\left(\boldsymbol{V}_{\mathbf{C}}\right)}\right)
\end{gathered}
$$

$=\sum_{a} \frac{-m_{a} \cdot c}{\gamma\left(\boldsymbol{V}_{\mathbf{C}}\right)}\left(g_{0 \alpha^{*}}{ }^{*}\left(\boldsymbol{r}_{\boldsymbol{a}}^{*}, t^{*}\right) \frac{d x_{a}^{\alpha *}}{d s} c+g_{\alpha \beta}{ }^{*}\left(\boldsymbol{r}_{\boldsymbol{a}}^{*}, t^{*}\right) \frac{d x_{a}^{\beta *}}{d t^{*}} \frac{d x_{a}^{\alpha *}}{d s}\right)+\left(\gamma\left(\boldsymbol{V}_{\mathbf{C}}\right) \frac{E^{*}}{c^{2}} \boldsymbol{V}_{\mathbf{C}}\right) \boldsymbol{V}_{\boldsymbol{c}}-\left(\frac{\sum_{a}\left[-m_{a} \cdot c \sqrt{g_{i k^{*}}\left(x_{a}^{j *}\right) \frac{d x_{a}^{i *}}{d t^{*}} \frac{d x_{a}^{k *}}{d t^{*}}}\right]}{\gamma\left(\boldsymbol{V}_{\mathbf{C}}\right)}\right)$

$=\sum_{a} \frac{-m_{a} \cdot c}{\gamma\left(\boldsymbol{V}_{\mathbf{C}}\right)}\left(g_{i \alpha}^{*}\left(\boldsymbol{r}_{\boldsymbol{a}}^{*}, t^{*}\right) \frac{d x_{a}^{\alpha *}}{d t^{*}} \frac{d x_{a}^{i *}}{d s}\right)+\gamma\left(\boldsymbol{V}_{\mathbf{C}}\right) E^{*} \beta^{2}-\left(\frac{\sum_{a}\left[-m_{a} \cdot c d s_{a}\right]}{\gamma\left(\boldsymbol{V}_{\mathbf{C}}\right) d t^{*}}\right)$

$=\sum_{a} \frac{-m_{a} \cdot c}{\gamma\left(\boldsymbol{V}_{\mathbf{C}}\right)}\left(g_{i k}^{*}\left(\boldsymbol{r}_{a}^{*}, t^{*}\right) \frac{d x_{a}^{k *}}{d t^{*}} \frac{d x_{a}^{i *}}{d s}-g_{i 0}{ }^{*}\left(\boldsymbol{r}_{\boldsymbol{a}}^{*}, t^{*}\right) \frac{d x_{a}^{i *}}{d s} \frac{d x_{a}^{0 *}}{d t^{*}}\right)+\gamma\left(\boldsymbol{V}_{\mathbf{C}}\right) E^{*} \beta^{2}-\left(\frac{\sum_{a}\left[-m_{a} \cdot c d s_{a}\right]}{\gamma\left(\boldsymbol{V}_{\mathbf{C}}\right) d t^{*}}\right)$

$=\sum_{a} \frac{-m_{a} \cdot c}{\gamma\left(\boldsymbol{V}_{\mathrm{C}}\right)}\left(\frac{d s}{d t^{*}}-u_{0}^{*}\left(\boldsymbol{r}_{a}^{*}, t^{*}\right) \frac{d x_{a}^{0 *}}{d t^{*}}\right)+\gamma\left(\boldsymbol{V}_{\mathbf{C}}\right) E^{*} \beta^{2}-\left(\frac{\sum_{a}\left[-m_{a} \cdot c d s\right]}{\gamma\left(\boldsymbol{V}_{\mathbf{C}}\right) d t^{*}}\right)$

$=\sum_{a} \frac{-m_{a} \cdot c}{\gamma\left(\boldsymbol{V}_{\mathbf{C}}\right)}\left(-u_{0}^{*}\left(\boldsymbol{r}_{\boldsymbol{a}}^{*}, t^{*}\right) \frac{d x_{a}^{0 *}}{d t^{*}}\right)+\gamma\left(\boldsymbol{V}_{\mathbf{C}}\right) E^{*} \beta^{2}$

$=\sum_{a} \frac{m_{a} \cdot c u_{0}^{*}\left(\boldsymbol{r}_{\boldsymbol{a}}^{*}, t^{*}\right)}{\gamma\left(\boldsymbol{V}_{\mathbf{C}}\right)}+\gamma\left(\boldsymbol{V}_{\mathbf{C}}\right) E^{*} \beta^{2}=\frac{E^{*}}{\gamma\left(\boldsymbol{V}_{\mathbf{C}}\right)}+\gamma\left(\boldsymbol{V}_{\mathbf{C}}\right) E^{*} \beta^{2}=\gamma\left(\boldsymbol{V}_{\mathbf{C}}\right) E^{*}\left(\frac{1}{\gamma\left(\boldsymbol{V}_{\mathbf{C}}\right)^{2}}+\beta^{2}\right)=\gamma\left(\boldsymbol{V}_{\mathbf{C}}\right) E^{*}\left(1-\beta^{2}+\beta^{2}\right)=\gamma\left(\boldsymbol{V}_{\mathbf{C}}\right) E^{*}$ 


$$
E=\gamma E^{*}
$$

With

$$
\begin{aligned}
E^{*}=\sum_{a} E_{a}^{*}= & \sum_{a} m_{a} \cdot c^{2} g_{0 i}{ }^{*}\left(\boldsymbol{r}_{\boldsymbol{a}}^{*}, t^{*}\right) u_{a}^{i *} \\
& =\left(\sum_{a} \gamma\left(\frac{d \boldsymbol{r}_{\boldsymbol{a}}^{*}}{d s_{a}}\right)^{*} m_{a} \cdot c^{2} \sqrt{g_{00}{ }^{*}\left(\boldsymbol{r}_{\boldsymbol{a}}^{*}\right)}\right)_{\text {if stationary: } d \tau^{*}=\frac{\sqrt{g_{00}^{*}}}{c}\left(d x_{0}{ }^{*}+\frac{g_{0 \alpha^{*}}}{g_{00}} d x^{\alpha^{*}}\right)}
\end{aligned}
$$

Thus we can also again write

$$
E=\gamma M c^{2}
$$

We see here that :

- as the Gauge field theory, if we change the coordinate system, $M$ is modified but not the role of the mass as again an origin of the Energy scale (accordance with Landau-Lifchitz [1]), which remain:

○ for every gravitational field;

- for every coordinate space-time.

- But contrary to the Gauge field theory, the mass is not a sum of :
- a free part;
○ plus an interacting part ;
$\circ$ plus an gauge part.

Indeed as the system is always and necessary in the space-time there is no way to separate it from the gravitational field: there is no free term relative to the gravitation, only interacting terms. This is in accordance with the fundamental and particular role of gravitation in physics. Otherwise any terms in the action would lose its signification due to the arbitrariness of the coordinate system: the truly role of the gravitation (via the metric) is the one of a filter which absorbs any effect of the coordinate transformation in the action.

Although the mass depends of the context (Einstein-Mach idea), in practice we work in a cosmological context where for quasi-every system, if we move sufficiently away from it, the spacetime tends to be approximatively Minkowskian. Thus, in paragraph 96 of [1], Landau-Lifchitz have given the proof that the 4-momentum of the whole system (particles with gravitation field), and so the mass, are independent of the frame of reference if the whole system studied is "free" (三in a outside Minkowskian gravitational field. So the total mass in an internal volume surround by a Galilean domain are well defined. It is again a "cosmological" accident in the RG spirit. 


\section{Why the Einstein law (the mass as internal energy) does not appear in Newtonian mechanics?} The crucial role of the Einstein non-universality of time law

If we put from the start of the theory the Newtonian law that time is universal, $d t=d t^{*}$, that is to say $\gamma\left(V_{\mathrm{c}}\right)=1$, we have:

$$
\boldsymbol{P}_{\boldsymbol{c}} \equiv \frac{\partial L^{\prime}}{\partial \boldsymbol{V}_{\mathbf{C}}}=\frac{\partial L^{\prime}\left(\left\{\boldsymbol{r}_{\boldsymbol{a}}^{*}\right\},\left\{\frac{d \boldsymbol{r}_{\boldsymbol{a}}^{*}}{d t}\right\}, \boldsymbol{R}_{\mathbf{C}}, \boldsymbol{V}_{\mathbf{C}}\right)}{\partial \boldsymbol{V}_{\mathbf{C}}}=\frac{\partial}{\partial \boldsymbol{v}_{\mathrm{C}}} \frac{L^{*}\left(\left\{\boldsymbol{r}_{\boldsymbol{a}}^{*}\right\},\left\{\gamma\left(\boldsymbol{V}_{\mathrm{C}}\right) \frac{d \boldsymbol{r}_{\boldsymbol{a}}^{*}}{d t}\right\}\right)}{\gamma\left(\boldsymbol{V}_{\mathrm{C}}\right)}=\frac{\partial}{\partial \boldsymbol{V}_{\mathrm{C}}} L^{*}\left(\left\{\boldsymbol{r}_{\boldsymbol{a}}^{*}\right\}, \frac{d \boldsymbol{r}_{\boldsymbol{a}}^{*}}{d t}\right)=0
$$

Which is of course wrong, so why can't we use the Lagrangian $L^{\prime}\left(\left\{\boldsymbol{r}_{\boldsymbol{a}}^{*}\right\},\left\{\frac{d \boldsymbol{r}_{a}^{*}}{d t}\right\}, \boldsymbol{R}_{\mathbf{C}}, \boldsymbol{V}_{\mathbf{C}}\right)$ ?

Actually this Lagrangian is correct...the problem is the passage to the limit:

- Einsteinian non universality of time $d t \neq d t^{*}$,

○ To the Newtonian universality of time $d t=d t^{*}$,

- Before the derivation and not after.

Indeed, if keep the non universality of time during the derivation process we have necessary directly

$$
\boldsymbol{P}_{c, \text { Einstein }}=\boldsymbol{V}_{\mathrm{C} \cdot} \cdot \gamma\left(\boldsymbol{V}_{\mathrm{C}}^{2}\right) \frac{E^{*}}{c^{2}}
$$

And then, the passage to the limit of universality of time gives

$$
\boldsymbol{P}_{c, \text { Newton }}=\lim _{\gamma\left(V_{\mathrm{c}}{ }^{2} \rightarrow 1\right.} \boldsymbol{V}_{\mathrm{C}} \cdot \gamma\left(\boldsymbol{V}_{\mathrm{C}}^{2}\right) \frac{E^{*}}{c^{2}}=\boldsymbol{V}_{\mathrm{C}} \cdot \frac{E^{*}}{c^{2}}
$$

Which keep the link between the mass and the internal energy in the Newtonian limit since the proportionality coefficient is again $\frac{E^{*}}{c^{2}}$.

Therefore, we are faced to a (famous) mathematical non equivalence (non-commutativity of derivative and the limit operation):

$$
\left[\frac{\partial\left(\lim _{\gamma\left(\boldsymbol{V}_{\mathbf{C}}\right) \rightarrow 1} L^{\prime}\right)}{\partial \boldsymbol{V}_{\mathbf{C}}}=\frac{\partial}{\partial \boldsymbol{V}_{\mathbf{C}}}\left(\lim _{\gamma\left(\boldsymbol{V}_{\mathbf{C}}\right) \rightarrow 1} \frac{L^{*}\left(\left\{\boldsymbol{r}_{\boldsymbol{a}}^{*}\right\},\left\{\gamma\left(\boldsymbol{V}_{\mathbf{C}}\right) \frac{d \boldsymbol{r}_{a}^{*}}{d t}\right\}\right)}{\gamma\left(\boldsymbol{V}_{\mathbf{C}}\right)}\right)\right] \neq\left[\lim _{\gamma\left(\boldsymbol{V}_{\mathbf{C}}\right) \rightarrow 1}\left(\frac{\partial L^{\prime}}{\partial \boldsymbol{V}_{\mathbf{C}}}\right)=\lim _{\gamma\left(\boldsymbol{V}_{\mathbf{C}}\right) \rightarrow 1}\left(\frac{\partial}{\partial \boldsymbol{V}_{\mathbf{C}}} \frac{\left.L^{*}\left(\left\{\boldsymbol{r}_{\boldsymbol{a}}^{*}\right\},\left\{\gamma\left(\boldsymbol{V}_{\mathbf{C}}\right) \frac{d \boldsymbol{r}_{a}^{*}}{d t}\right\}\right)\right)}{\gamma\left(\boldsymbol{V}_{\mathbf{C}}\right)}\right)\right]
$$

So, what is the procedure used in the Newtonian theory, and why does the procedure not show the connection between mass and internal energy?

As explained in Landau-Lifchitz [2]:

$\circ$ we start from a Lagrangian $L\left(\left\{\boldsymbol{r}_{\boldsymbol{a}}\right\},\left\{\frac{d \boldsymbol{r}_{\boldsymbol{a}}}{d t}\right\}, t\right)$

$\circ$ we pose the principle of the additivity of the Lagrangian for independent system (as in Einstein Special Relativity) (indeed without this there would be non-local behaviour)

$$
L\left(\left\{\boldsymbol{r}_{\boldsymbol{a}}\right\},\left\{\frac{d \boldsymbol{r}_{\boldsymbol{a}}}{d t}\right\}, t\right)=\sum L\left(\boldsymbol{r}_{\boldsymbol{a}}, \frac{d \boldsymbol{r}_{\boldsymbol{a}}}{d t}, t\right)
$$

○ we pose the principle of the homogeneity of space and for time

$$
L\left(\boldsymbol{r}_{\boldsymbol{a}}, \frac{d \boldsymbol{r}_{\boldsymbol{a}}}{d t}, t\right)=L\left(\frac{d \boldsymbol{r}_{\boldsymbol{a}}}{d t}\right)
$$


- we pose the principle of the isotropy of space

$$
L\left(\frac{d \boldsymbol{r}_{\boldsymbol{a}}}{d t}\right)=L\left(\left(\frac{d \boldsymbol{r}_{a}}{d t}\right)^{2}\right)
$$

0 we pose the principle of Galileo-Newtonian kinematics between Galilean frames K \& K'

$$
\left(\begin{array}{c}
t=t^{\prime} \text { (universality of time) } \\
x=x^{\prime}+V_{K^{\prime} / K} \cdot t^{\prime} \\
y=y^{\prime} \\
z=z^{\prime}
\end{array}\right) \text { which implies the additivity formula }\left(\frac{d \boldsymbol{r}_{\boldsymbol{a}}}{d t}=\frac{d \boldsymbol{r}_{\boldsymbol{a}}{ }^{\prime}}{d t}+\boldsymbol{V}_{\boldsymbol{K}^{\prime} / \boldsymbol{K}}\right)
$$

Therefore we can compute the momentum where $\mathrm{K}^{\prime}=\mathrm{K}^{*}$ and $\boldsymbol{V}_{\boldsymbol{K}^{\prime} / \boldsymbol{K}}=\boldsymbol{V}_{\boldsymbol{c}}$

$$
\begin{aligned}
& \boldsymbol{P}_{\boldsymbol{c}, \text { Newton }}=\frac{\partial}{\partial \boldsymbol{V}_{\mathbf{C}}} L\left(\left\{\boldsymbol{r}_{\boldsymbol{a}}\right\},\left\{\frac{d \boldsymbol{r}_{\boldsymbol{a}}}{d t}\right\}\right)=\frac{\partial}{\partial \boldsymbol{V}_{\mathbf{C}}} \sum L\left(\frac{d \boldsymbol{r}_{\boldsymbol{a}}{ }^{2}}{d t}\right)=\sum \frac{\partial}{\partial \boldsymbol{V}_{\mathbf{C}}} L\left(\left(\frac{d \boldsymbol{r}_{\boldsymbol{a}}^{*}}{d t}+\boldsymbol{V}_{\mathbf{C}}\right)^{2}\right) \\
& =\sum \frac{\partial\left(\frac{d \boldsymbol{r}_{\boldsymbol{a}}^{*}}{d t}+\boldsymbol{V}_{\mathbf{C}}\right)^{2}}{\partial \boldsymbol{V}_{\mathbf{C}}} \frac{\partial}{\partial \frac{d \boldsymbol{r}_{\boldsymbol{a}}{ }^{2}}{d t}} L\left(\frac{d \boldsymbol{r}_{\boldsymbol{a}}{ }^{2}}{d t}\right)=\sum 2\left(\frac{d \boldsymbol{r}_{\boldsymbol{a}}^{*}}{d t}+\boldsymbol{V}_{\mathbf{C}}\right) \frac{\partial}{\partial \frac{d \boldsymbol{r}_{\boldsymbol{a}}{ }^{2}}{d t}} L\left(\frac{d \boldsymbol{r}_{\boldsymbol{a}}{ }^{2}}{d t}\right) \\
& =\sum 2\left(\frac{d r_{a}^{*}}{d t}\right) \frac{\partial}{\partial \frac{d r_{a}^{2}}{d t}} L\left(\frac{d r_{a}^{2}}{d t}\right)+2 V_{\mathbf{C}} \sum \frac{\partial}{\partial \frac{d r_{a}{ }^{2}}{d t}} L\left(\frac{d r_{a}^{2}}{d t}\right)=\sum\left(\frac{d r_{a}^{*}}{d t}\right) \lambda_{a}+V_{\mathbf{C}} \sum \lambda_{a}
\end{aligned}
$$

- if we define $\mathrm{K}^{*}$ such that $\sum\left(\frac{d r_{a}^{*}}{d t}\right) \lambda_{a} \equiv 0$

- and the quantities $\lambda_{a}\left(\frac{d r_{a}}{d t}\right) \equiv 2 \frac{\partial}{\partial \frac{d r_{a}}{d t}} L\left(\frac{d r_{a}^{2}}{d t}\right)$ a priori not constant (the future mass).

In conclusion with first Galileo-Newtonian principles we have, without any other hypothesis:

- Homogeneity \& isotropy of space and homogeneity of time

- \& Kinematic Galileo-Newtonian (Galilean transformation )

- \& Additivity of the Lagrangian (for independent system)

$$
=>\boldsymbol{P}_{c, \text { Newton }}=\frac{\partial}{\partial \boldsymbol{v}_{\mathbf{C}}} L\left(\left\{\frac{d \boldsymbol{r}_{\boldsymbol{a}}}{d t}\right\}\right)=\left(\sum \lambda_{a}\right) \boldsymbol{v}_{\mathbf{C}}
$$

With

○ $\lambda_{a} \equiv 2 \frac{\partial}{\partial \frac{d r_{a}{ }^{2}}{d t}} L\left(\frac{d r_{a}^{2}}{d t}\right)$

$\circ\left(\sum\left(\frac{d r_{a}^{*}}{d t}\right) \lambda_{a} \equiv 0\right)_{\text {by defintion of } a K^{*}}$

In order to complete the mechanical description, we have to express the Lagrangian of a material point $L\left(\frac{d r_{a}^{2}}{d t}\right)$ more explicitly. For that we will call (following again [2]) another principle: the Galilean principle of Relativity which affirm that the mechanical law have to be the same for any Galilean frame $K^{\prime} K^{\prime}, K^{*} \ldots$ : The Lagrangian function $L$ have to be the same for any Galilean frame.

But, there is a problem, the only Galilean invariants are:

- The action $S\left[\left\{\boldsymbol{r}_{\boldsymbol{a}}^{*}\left(t^{*}\right)\right\}, \boldsymbol{R}_{\boldsymbol{c}}(t)\right]$ by construction (the quantity, not necessary the function!)

- The Newtonian time $d t=d t^{\prime}$

- And....nothing else 
We cannot construct an invariant with the basic kinematics quantities of a particle $\boldsymbol{r}_{\boldsymbol{a}}, \frac{d \boldsymbol{r}_{\boldsymbol{a}}}{d t}$ and so we cannot construct an invariant quantity $\left(\frac{d r_{a}{ }^{2}}{d t}\right) \cdot d t$.

So we are a priori blocked. In fact, the only possibility is to use the "Gauge invariance" associated to the least action principle,

- $\quad L\left(\frac{d \boldsymbol{r}_{a}{ }^{2}}{d t}\right)=L^{*}\left(\frac{d \boldsymbol{r}_{a}{ }^{2}}{d t}\right)+\frac{d f\left(\boldsymbol{r}_{a}^{*}, \boldsymbol{t}\right)}{d t}$

- that we complete by the Galilean principle of Relativity $L^{*}\left(\frac{d r_{a}{ }^{* 2}}{d t}\right)=L\left(\frac{d r_{a}^{* 2}}{d t}\right)$

(Remark: Einstein tells us that this Galilean principles contains also "the principle of GalileoNewtonian kinematics" defined above).

Thanks to this "Gauge invariance", we can make the following calculation:

$$
L\left(\frac{d \boldsymbol{r}_{\boldsymbol{a}}{ }^{2}}{d t}\right)=L\left(\left(\frac{d \boldsymbol{r}_{\boldsymbol{a}}^{*}}{d t}+\boldsymbol{v}_{\mathrm{C}}\right)^{2}\right)=L\left(\left(\frac{d \boldsymbol{r}_{\boldsymbol{a}}^{*}}{d t}\right)^{2}+\left(\boldsymbol{v}_{\mathrm{C}}\right)^{2}+2 \frac{d \boldsymbol{r}_{\boldsymbol{a}}^{*}}{d t} \boldsymbol{V}_{\mathrm{C}}\right)
$$

The expression, should be valid for any constant $\boldsymbol{V}_{\mathbf{C}}$, and so even for infinitesimal value $\boldsymbol{\varepsilon}$, then the following reasoning must be true:

$$
\begin{aligned}
& L\left(\frac{d \boldsymbol{r}_{\boldsymbol{a}}{ }^{2}}{d t}\right)=L\left(\left(\frac{d \boldsymbol{r}_{\boldsymbol{a}}^{*}}{d t}\right)^{2}+\boldsymbol{\varepsilon}^{2}+2 \frac{d \boldsymbol{r}_{\boldsymbol{a}}^{*}}{d t} \boldsymbol{\varepsilon}\right) \\
\approx & L\left(\frac{d \boldsymbol{r}_{\boldsymbol{a}}^{* 2}}{d t}\right)+\frac{d \boldsymbol{r}_{\boldsymbol{a}}^{*}}{d t} \varepsilon 2 \frac{\partial L}{\partial\left(\frac{d \boldsymbol{r}_{a}^{*}}{d t}\right)^{2}}\left(\left(\frac{d \boldsymbol{r}_{\boldsymbol{a}}^{*}}{d t}\right)^{2}\right) \\
= & L\left(\frac{d \boldsymbol{r}_{\boldsymbol{a}}^{* 2}}{d t}\right)+\frac{d \varepsilon \cdot \boldsymbol{r}_{\boldsymbol{a}}^{*}}{d t} 2 \frac{\partial L}{\partial\left(\frac{d \boldsymbol{r}_{\boldsymbol{a}}^{*}}{d t}\right)^{2}}\left(\left(\frac{d \boldsymbol{r}_{\boldsymbol{a}}^{*}}{d t}\right)^{2}\right) \\
= & L\left(\frac{d \boldsymbol{r}_{\boldsymbol{a}}^{* 2}}{d t}\right)+\lambda_{a} \frac{d \varepsilon \cdot \boldsymbol{r}_{a}^{*}}{d t} \text { with } \lambda_{a}=\lambda_{a}\left(\left(\frac{d \boldsymbol{r}_{\boldsymbol{a}}^{*}}{d t}\right)^{2}\right)
\end{aligned}
$$

Galilean Relativity principle $=>L\left(\frac{d r_{a}{ }^{2}}{d t}\right)=L^{*}\left(\frac{d r_{a}{ }^{2}}{d t}\right)=>L^{*}\left(\frac{d r_{a}{ }^{2}}{d t}\right)=L^{*}\left(\frac{d r_{a}{ }^{2}}{d t}\right)+\lambda_{a}{ }^{*} \frac{d \varepsilon . r_{a}}{d t}$

And the "Gauge invariance" $=>L^{*}\left(\frac{d \boldsymbol{r}_{a}{ }^{* 2}}{d t}\right)+\lambda_{a}{ }^{*} \frac{d \varepsilon \cdot \boldsymbol{r}_{a}{ }^{*}}{d t}=L^{*}\left(\frac{d \boldsymbol{r}_{a}{ }^{* 2}}{d t}\right)+\frac{d f\left(\boldsymbol{r}_{a}{ }^{*}\right)}{d t}$

$\Rightarrow>\frac{d f\left(\boldsymbol{r}_{a}{ }^{*}\right)}{d t}=\lambda_{a}{ }^{*} \frac{d \varepsilon \cdot \boldsymbol{r}_{\boldsymbol{a}}{ }^{*}}{d t}=\lambda_{a}{ }^{*} \frac{d g\left(\boldsymbol{r}_{\boldsymbol{a}}{ }^{*}\right)}{d t}=>\frac{\partial f\left(\boldsymbol{r}_{\boldsymbol{a}}{ }^{*}\right)}{\partial \boldsymbol{r}_{\boldsymbol{a}}{ }^{*}}=\lambda_{a}{ }^{*} \frac{\partial \varepsilon \boldsymbol{r}_{\boldsymbol{a}}{ }^{*}}{\partial \boldsymbol{r}_{\boldsymbol{a}}{ }^{*}}=>\lambda_{a}{ }^{*}=f\left(\boldsymbol{r}_{\boldsymbol{a}}{ }^{*}\right)$

But at the same time $\lambda_{a}{ }^{*}=\lambda_{a}{ }^{*}\left(\left(\frac{d \boldsymbol{r}_{a}{ }^{*}}{d t}\right)^{2}\right)$, then $\lambda_{a}{ }^{*}=$ cte, $\forall \boldsymbol{r}_{\boldsymbol{a}}{ }^{*} \forall \frac{d \boldsymbol{r}_{a}{ }^{*}}{d t} \forall t$

The constant Lagrangian characteristic coefficents $\lambda_{a}{ }^{*} \equiv 2 \frac{\partial}{\partial \frac{d r_{a}{ }^{*}}{d t}} L^{*}\left(\frac{d r_{a}{ }^{2}}{d t}\right)=2 \frac{\partial}{\partial \frac{d r_{a}^{2}}{d t}} L\left(\frac{d r_{a}{ }^{2}}{d t}\right)=\lambda_{a}$ are what we call the mass $m_{a}$ of a material point. 
It results from that, the expression of the momentum of the center of mass.

$$
\boldsymbol{P}_{c, \text { Newton }}=\left(\sum \lambda_{a}\right) \boldsymbol{v}_{\mathrm{C}}=\left(\sum m_{a}\right) \boldsymbol{v}_{\mathrm{C}}=M . \boldsymbol{v}_{\mathrm{C}}
$$

This result à la Landau [2] shows us:

- the crucial role playing by the indeterminacy of the Lagrangian (the "Gauge invariance");

- And no more the existence of an invariant L.dt in the action.

This is the complete opposite of the Einsteinian case where:

- the invariant of the action was used in the start of the reasoning;

$\circ$ and the indeterminacy of the Lagrangian was kept away.

Moreover the latter "Gauge invariance", when taken into account afterwards in Relativity, was responsible for the undesirable change in the expression for mass (although the link between mass and the origin of the energy scale still remains). The consequence of this Newtonian inversion of the role between "Gauge invariance" and action invariance has 2 impacts in the description of the Newtonian mechanics.

a. The loss of connexion between the mass and the origin of the energy scale Indeed, from above, we compute the Lagrangian and find:

$$
\begin{aligned}
& L\left(\left\{\boldsymbol{r}_{\boldsymbol{a}}\right\},\left\{\frac{d \boldsymbol{r}_{\boldsymbol{a}}}{d t}\right\}\right) \\
& =\sum \frac{1}{2} m_{a}\left(\frac{d \boldsymbol{r}_{\boldsymbol{a}}{ }^{2}}{d t}\right)=\sum \frac{1}{2} m_{a}\left(\frac{d \boldsymbol{r}_{\boldsymbol{a}}{ }^{*}}{d t}+\boldsymbol{V}_{\mathbf{C}}\right)^{2} \\
& =\sum_{2}^{1} m_{a}\left(\left(\frac{d \boldsymbol{r}_{\boldsymbol{a}}{ }^{*}}{d t}\right)^{2}+\boldsymbol{V}_{\mathbf{C}}{ }^{2}+2 \frac{d \boldsymbol{r}_{\boldsymbol{a}}{ }^{*}}{d t} \boldsymbol{V}_{\mathbf{C}}\right) \\
& =\sum \frac{1}{2} m_{a}\left(\frac{d \boldsymbol{r}_{\boldsymbol{a}}}{d t}\right)^{2}+\frac{1}{2} M \boldsymbol{V}_{\mathbf{C}}{ }^{2} \\
& =>L^{\prime}\left(\left\{\boldsymbol{r}_{\boldsymbol{a}}{ }^{*}\right\},\left\{\frac{d \boldsymbol{r}_{\boldsymbol{a}}{ }^{*}}{d t}\right\}, \boldsymbol{R}_{\mathbf{C}}, \boldsymbol{V}_{\mathbf{C}}\right)=\sum \frac{1}{2} m_{a}\left(\frac{d \boldsymbol{r}_{\boldsymbol{a}}}{d t}\right)^{2}+\frac{1}{2} M \boldsymbol{V}_{\mathbf{C}}{ }^{2}
\end{aligned}
$$

The resulting energy expression is:

$$
\begin{aligned}
E^{\prime} \equiv \sum_{a} \frac{\partial L^{\prime}}{\partial \frac{d r_{a}^{*}}{d t} \frac{d \boldsymbol{r}_{a}^{*}}{d t}+\frac{\partial L^{\prime}}{\partial \boldsymbol{V}_{\mathbf{C}}} \boldsymbol{V}_{\boldsymbol{c}}}-L^{\prime}=\sum_{a} m_{a}\left(\frac{d \boldsymbol{r}_{\boldsymbol{a}}^{*}}{d t}\right)^{2}+M \cdot \boldsymbol{V}_{\mathbf{C}} \boldsymbol{V}_{\boldsymbol{c}}-\sum \frac{1}{2} m_{a}\left(\frac{d \boldsymbol{r}_{\boldsymbol{a}}^{*}}{d t}\right)^{2}-\frac{1}{2} M \boldsymbol{V}_{\mathbf{C}}{ }^{2} \\
=>E^{\prime}=\sum \frac{1}{2} m_{a}\left(\frac{d \boldsymbol{r}_{\boldsymbol{a}}{ }^{*}}{d t}\right)^{2}+\frac{1}{2} M \boldsymbol{V}_{\mathbf{C}}{ }^{2}
\end{aligned}
$$

The mass no longer defines the origin of the energy scale.

b. the unnatural fixation of the origin of the energy scale by relativistic invariance

Since, we do not use a relativistic invariant quantity to express the Lagrangian, we obviously cannot use it to fix of the Lagrangian expression with relativist invariance. 
Thus the Lagrangian is only relative to a gauge, so this is also the case for the origin of the energy scale.

$$
L_{\text {modif }}^{\prime}\left(\left\{\boldsymbol{r}_{\boldsymbol{a}}{ }^{*}\right\},\left\{\frac{d \boldsymbol{r}_{\boldsymbol{a}}{ }^{*}}{d t}\right\}, \boldsymbol{R}_{\mathbf{C}}, \boldsymbol{V}_{\mathbf{C}}\right)=L^{\prime}\left(\left\{\boldsymbol{r}_{\boldsymbol{a}}{ }^{*}\right\},\left\{\frac{d \boldsymbol{r}_{\boldsymbol{a}}{ }^{*}}{d t}\right\}, \boldsymbol{R}_{\mathbf{C}}, \boldsymbol{V}_{\mathbf{C}}\right)+\sum_{a} \frac{d f\left(\boldsymbol{r}_{\boldsymbol{a}}{ }^{*}, t\right)}{d t}
$$

This indeterminacy "contaminates" that of the origin of the energy scale:

$$
\begin{aligned}
& =>E_{\text {modif }}^{\prime} \equiv \sum_{a} \frac{\partial L_{\text {modif }}^{\prime}}{\partial \frac{d \boldsymbol{r}_{\boldsymbol{a}}^{*}}{d t}} \frac{d \boldsymbol{r}_{\boldsymbol{a}}^{*}}{d t}+\frac{\partial L_{\text {modif }}^{\prime}}{\partial \boldsymbol{V}_{\mathbf{C}}} \boldsymbol{V}_{\boldsymbol{c}}-L_{\text {modif }}^{\prime} \\
& =\sum_{a} \frac{\partial\left(L^{\prime}\left(\left\{\boldsymbol{r}_{a}^{*}\right\},\left\{\frac{d \boldsymbol{r}_{a}^{*}}{d t}\right\}, \boldsymbol{R}_{\mathrm{C}}, \boldsymbol{V}_{\mathbf{C}}\right)+\frac{d f\left(\boldsymbol{r}_{a}^{*}, t\right)}{d t}\right)}{\partial \frac{d \boldsymbol{r}_{a}^{*}}{d t}} \frac{d \boldsymbol{r}_{a}^{*}}{d t}+M . \boldsymbol{V}_{\mathbf{C}} \boldsymbol{V}_{\boldsymbol{c}}-L^{\prime}\left(\left\{\boldsymbol{r}_{a}{ }^{*}\right\},\left\{\frac{d \boldsymbol{r}_{\boldsymbol{a}}{ }^{*}}{d t}\right\}, \boldsymbol{R}_{\mathbf{C}}, \boldsymbol{V}_{\mathbf{C}}\right)-\frac{d f\left(\boldsymbol{r}_{a}^{*}, t\right)}{d t} \\
& =\sum_{a} m_{a}\left(\frac{d \boldsymbol{r}_{a}{ }^{*}}{d t}\right)^{2}+\sum_{a} \frac{\partial f\left(\boldsymbol{r}_{a}{ }^{*}, t\right)}{\partial \frac{d \boldsymbol{r}_{a}^{*}}{d t}} \frac{d \boldsymbol{r}_{a}^{*}}{d t}+M \cdot \boldsymbol{V}_{\mathrm{C}} \boldsymbol{V}_{c}{ }^{2}-L^{\prime}\left(\left\{\boldsymbol{r}_{\boldsymbol{a}}{ }^{*}\right\},\left\{\frac{d \boldsymbol{r}_{a}{ }^{*}}{d t}\right\}, \boldsymbol{R}_{\mathrm{C}}, \boldsymbol{V}_{\mathrm{C}}\right)-\sum_{a} \frac{d f\left(\boldsymbol{r}_{a}{ }^{*}, t\right)}{d t} \\
& =E^{\prime}+\sum_{a} \frac{\partial f\left(\boldsymbol{r}_{a}{ }^{*}, t\right)}{\partial \frac{d \boldsymbol{r}_{a}^{*}}{d t}} \frac{d \boldsymbol{r}_{a}^{*}}{d t}-\sum_{a} \frac{d f\left(\boldsymbol{r}_{a}{ }^{*}, t\right)}{d t}=E^{\prime}-\sum_{a} \frac{\partial f\left(\boldsymbol{r}_{a}{ }^{*}, t\right)}{\partial t} \\
& E_{\text {modif }}^{\prime}=E^{\prime}-\sum_{a} \frac{\partial f\left(\boldsymbol{r}_{\boldsymbol{a}}{ }^{*}, t\right)}{\partial t}
\end{aligned}
$$

\section{Summary:}

Einstein's law (internal-)energy-mass equivalence law comes from Einstein's low of non-universality of time law by the fact it gives the existence of invariants in the action, this also has 2 other consequences, the fixation of the energy scale by the mass and the fixing of the value of the mass (in free system) by the natural demand of the Lagrangian L.dt invariant expression in the action (by saying that the "Gauge invariance" although allowed, is "not natural").

The Newtonian universality of time completely reverses the situation. There are no more sufficiently invariant quantities, this obliges us to use the "unnatural Gauge invariance" of the action, which hides Einstein's law and suppress the role of the mass as the origin of the energy scale. Moreover, this necessary use of "gauge invariance" does not allow us to talk about (and even think of) a natural invariant L.dt expression which was so necessary to define the origin of the energy scale in a "natural" way.

Worst, this frequent use of "gauge invariance" in Newtonian mechanic has accustomed us to consider that the energy scale has "no natural" fixed value (in free system). Therefore when Einstein discovered-invented the Special Relativity in 1905 and the mass-(Internal-)energy equivalence, he was again conditioned by this habit and so hesitated to set a fixed value of the origin of the energy scale by the mass. He waited several years before fixing it (cf. [7] or the original Einstein's articles like [15] where he talked about difference of energy, instead of the "absolute" energy). But he set the origin of the energy scale via an intuition of the naturalness rather by than a Lagrangian explanation. Lagrangian point of view was (at that time) not at all the only formal expression of physic laws, it was surely not as mature as today (Noether Theorem, Schwarzchild electromagnetic Lagrangian, EinsteinHilbert gravitational action, Standard Model action etc, and even in the future quantum mechanics as Feynman showed us) and so a priori not the unique convincing road to physics. I suppose that, if he was more confident on the general validity of it (not evident a priori), he would have proposed probably a Lagrangian proof, even if he used it after many times in Special and General Relativity and on its others modifications of his theory. 


\section{The energy-momentum tensor and the mass as a scalar}

A simple Lorentz transformation, shows that the 3-momentum is actually the one associated to the 4-vector defined above $P^{i}\left(K^{*}\right)=\frac{1}{c} \iiint \int_{\text {space-tim }} T^{i k} \delta\left(n_{l m} x^{l} x^{m}\right) \cdot d \eta_{k}\left(K^{*}\right) d^{4} x$.

Thus, among all the 4-momentum $P^{i}(K), P^{i}\left(K^{\prime}\right), P^{i}\left(K^{*}\right) \ldots$ the Lagrangian method selects $P^{i}\left(K^{*}\right)$.

Moreover, thanks to this association we can naturally affirm that the mass, and so the internal energy, is a scalar: this is the well known norm of the 4-momentum. 


\section{Conclusion}

We have a way to demonstrate the famous Einstein formula $\mathrm{E}^{*}=\mathrm{Mc}^{2}$ directly from an appropriate Lagrangian function selecting the correct variables.

Instead of $L\left(\left\{\boldsymbol{r}_{\boldsymbol{a}}\right\},\left\{\frac{d \boldsymbol{r}_{\boldsymbol{a}}}{d t}\right\}\right)$, we use $L^{\prime}\left(\left\{\boldsymbol{r}_{\boldsymbol{a}}^{*}\right\},\left\{\frac{d \boldsymbol{r}_{a}^{*}}{d t}\right\}, \boldsymbol{R}_{\mathbf{C}}, \boldsymbol{V}_{\mathbf{C}}\right)=\frac{L^{*}\left(\left\{\boldsymbol{r}_{\boldsymbol{a}}^{*}\right\},\left\{\gamma\left(\boldsymbol{V}_{\mathbf{C}}\right) \frac{d \boldsymbol{r}_{a}^{*}}{d t}\right\}\right)}{\gamma\left(\boldsymbol{V}_{\mathbf{C}}\right)}$.

Instead of $L\left[\{\varphi\},\left\{\frac{\partial \varphi}{\partial r}\right\},\left\{\frac{\partial \varphi}{\partial t}\right\}\right]$, we use $L^{\prime}\left[\left\{\varphi^{*}\right\},\left\{\frac{\partial \varphi^{*}}{\partial r^{*}}\right\},\left\{\frac{\partial \varphi^{*}}{\partial t}\right\}, \boldsymbol{R}_{\mathbf{C}}, \boldsymbol{V}_{\mathbf{C}}\right] \equiv \frac{\iiint \Lambda^{*}\left(\varphi^{*}, \frac{\partial \varphi^{*}}{\partial r^{*}}, \gamma\left(V_{\mathrm{C}}\right) \frac{\partial \varphi^{*}}{\partial t}\right) d V^{*}}{\gamma\left(V_{\mathrm{C}}\right)}$.

In the two cases we've calculated directly that $\boldsymbol{P}_{\boldsymbol{c}} \equiv \frac{\partial L^{\prime}}{\partial \boldsymbol{V}_{\mathbf{C}}}=\gamma \frac{E^{*}}{c^{2}} \boldsymbol{V}_{\mathbf{C}}$

In this article, we also showed:

- The strong link with this law and the time dilation formula that highlight the crucial role of the Einstein's requirement of non-universality of time;

- A discussion on the meaning of the new set of variables chosen with an amusing modified velocity addition formula that does not contradict the of Einstein-Poincaré one;

- A discussion of the origin of the energy scale and the link to mass as stated by LandauLifchitz;

- Why in Newtonian mechanic Einstein's law is hidden;

- I also add some elements for a Hamiltonian analysis and a discussion about the model of electron that allows the formalism to be applied to a concrete example. 
- Hamiltonian map

The 4-momentum is $\quad P^{i}\left(K^{*}\right)=(M c, P)=\left(\gamma \frac{E^{*}}{c}, \gamma \frac{E^{*}}{c^{2}} \boldsymbol{V}_{\mathbf{C}}\right)$

Then

$$
\begin{gathered}
\left\|P^{i}\left(K^{*}\right)\right\|^{2}=\left(\frac{E}{c}\right)^{2}-\boldsymbol{P}^{2}=\left(\gamma \frac{E^{*}}{c}\right)^{2}-\left(\gamma \frac{E^{*}}{c^{2}} \boldsymbol{V}_{\mathbf{C}}\right)^{2}=\left(\gamma \frac{E^{*}}{c}\right)^{2}\left(1-\left(\frac{\boldsymbol{V}_{\mathbf{C}}}{1}\right)^{2}\right)=\left(\frac{E^{*}}{c}\right)^{2} \\
=>\left(\frac{E^{*}}{c}\right)^{2}=\left(\frac{E}{c}\right)^{2}-\boldsymbol{P}^{2} \\
<=>E^{2}=E^{* 2}+c^{2} \boldsymbol{P}^{2} \\
<=>E=\sqrt{E^{* 2}+c^{2} \boldsymbol{P}^{2}}
\end{gathered}
$$

Having also $E^{*}=\sum_{a} E_{a}^{*}=\sum_{a} \sqrt{\left(m_{a} c^{2}\right)^{2}+c^{2} \boldsymbol{P}_{\boldsymbol{a}}^{* 2}}$

$$
=>E=\sqrt{\left(\sum_{a} \sqrt{\left(m_{a} c^{2}\right)^{2}+c^{2} \boldsymbol{P}_{\boldsymbol{a}}^{* 2}}\right)^{2}+c^{2} \boldsymbol{P}^{2}}
$$

Thus the Hamiltonian map $H:\left(\left\{\boldsymbol{r}_{\boldsymbol{a}}^{*}\right\},\left\{\boldsymbol{P}_{\boldsymbol{a}}{ }^{*}\right\}, \boldsymbol{R}_{\mathbf{C}}, \boldsymbol{P}\right) \rightarrow H\left(\left\{\boldsymbol{r}_{\boldsymbol{a}}^{*}\right\},\left\{\boldsymbol{P}_{\boldsymbol{a}}{ }^{*}\right\}, \boldsymbol{R}_{\mathbf{C}}, \boldsymbol{P}\right) \equiv E$ is

$$
\begin{aligned}
H\left(\left\{\boldsymbol{r}_{\boldsymbol{a}}^{*}\right\},\left\{\boldsymbol{P}_{\boldsymbol{a}}^{*}\right\}, \boldsymbol{R}_{\mathbf{C}}, \boldsymbol{P}\right)= & \sqrt{H^{*}\left(\left\{\boldsymbol{r}_{\boldsymbol{a}}^{*}\right\},\left\{\boldsymbol{P}_{\boldsymbol{a}}^{*}\right\}\right)^{2}+c^{2} \boldsymbol{P}^{2}} \\
& \text { with } \\
H^{*}\left(\left\{\boldsymbol{r}_{\boldsymbol{a}}^{*}\right\},\left\{\boldsymbol{P}_{\boldsymbol{a}}^{*}\right\}\right)= & \sum_{a} \sqrt{\left(m_{a} c^{2}\right)^{2}+c^{2} \boldsymbol{P}_{\boldsymbol{a}}^{* 2}}
\end{aligned}
$$

With $H^{*}:\left(\left\{\boldsymbol{r}_{\boldsymbol{a}}^{*}\right\},\left\{\boldsymbol{P}_{\boldsymbol{a}}^{*}\right\}, \boldsymbol{R}_{\mathbf{C}}, \boldsymbol{P}\right) \rightarrow H^{*}\left(\left\{\boldsymbol{r}_{\boldsymbol{a}}^{*}\right\},\left\{\boldsymbol{P}_{\boldsymbol{a}}^{*}\right\}, \boldsymbol{R}_{\mathbf{C}}, \boldsymbol{P}\right) \equiv E^{*}$

I give below with evident notation 3 kinds of approximation:

- $H_{\{a\} \text { Newtonian }}\left(\left\{\boldsymbol{r}_{\boldsymbol{a}}^{*}\right\},\left\{\boldsymbol{P}_{\boldsymbol{a}}^{*}\right\}, \boldsymbol{R}_{\mathbf{C}}, \boldsymbol{P}\right)=\sqrt{H^{*}\left(\left\{\boldsymbol{r}_{\boldsymbol{a}}^{*}\right\},\left\{\boldsymbol{P}_{\boldsymbol{a}}^{*}\right\}\right)^{2}+c^{2} \boldsymbol{P}^{2}}$

with

$$
\begin{aligned}
H^{*}\left(\left\{\boldsymbol{r}_{\boldsymbol{a}}^{*}\right\},\left\{\boldsymbol{P}_{\boldsymbol{a}}^{*}\right\}\right) & =\sum_{a} \sqrt{\left(m_{a} c^{2}\right)^{2}+c^{2} \boldsymbol{P}_{\boldsymbol{a}}^{* 2}}=\sum_{a}\left(m_{a} c^{2}\right) \sqrt{1+\frac{c^{2} \boldsymbol{P}_{\boldsymbol{a}}^{* 2}}{\left(m_{a} c^{2}\right)^{2}}} \\
& \approx \sum_{a} m_{a} c^{2}\left(1+\frac{1}{2} \frac{c^{2} \boldsymbol{P}_{\boldsymbol{a}}{ }^{2}}{\left(m_{a} c^{2}\right)^{2}}\right)=M_{\Sigma} c^{2}+\sum_{a} \frac{\boldsymbol{P}_{\boldsymbol{a}}^{* 2}}{2 m_{a}}
\end{aligned}
$$




$$
\begin{aligned}
& =>H_{\{a\} \text { Newtonian }}\left(\left\{\boldsymbol{r}_{\boldsymbol{a}}^{*}\right\},\left\{\boldsymbol{P}_{\boldsymbol{a}}^{*}\right\}, \boldsymbol{R}_{\mathbf{C}}, \boldsymbol{P}\right)=\sqrt{\left(M_{\Sigma} c^{2}+\sum_{a} \frac{\boldsymbol{P}_{\boldsymbol{a}}{ }^{* 2}}{2 m_{a}}\right)^{2}+c^{2} \boldsymbol{P}^{2}} \\
& =\sqrt{\left(M_{\Sigma} c^{2}\right)^{2}\left(1+\frac{1}{M_{\Sigma} c^{2}} \sum_{a} \frac{\boldsymbol{P}_{\boldsymbol{a}}{ }^{2}}{2 m_{a}}\right)^{2}+c^{2} \boldsymbol{P}^{2}} \\
& \approx \sqrt{\left(M_{\Sigma} c^{2}\right)^{2}\left(1+\frac{2}{M_{\Sigma} c^{2}} \sum_{a} \frac{\boldsymbol{P}_{\boldsymbol{a}}^{* 2}}{2 m_{a}}\right)+c^{2} \boldsymbol{P}^{2}} \\
& =\sqrt{\left(M_{\Sigma} c^{2}\right)^{2}+c^{2} \sum_{a}\left(\frac{M_{\Sigma}}{m_{a}}\right) \boldsymbol{P}_{\boldsymbol{a}}^{* 2}+c^{2} \boldsymbol{P}^{2}}=\sqrt{\left(M_{\Sigma} c^{2}\right)^{2}+c^{2} \boldsymbol{P}^{2}} \sqrt{1+\frac{c^{2} \sum_{a}\left(\frac{M_{\Sigma}}{m_{a}}\right) \boldsymbol{P}_{\boldsymbol{a}}^{* 2}}{\left(M_{\Sigma} c^{2}\right)^{2}+c^{2} \boldsymbol{P}^{2}}} \\
& \approx \sqrt{\left(M_{\Sigma} c^{2}\right)^{2}+c^{2} \boldsymbol{P}^{2}}\left(1+\frac{1}{2} \frac{c^{2} \sum_{a}\left(\frac{M_{\Sigma}}{m_{a}}\right) \boldsymbol{P}_{\boldsymbol{a}}^{* 2}}{\left(M_{\Sigma} c^{2}\right)^{2}+c^{2} \boldsymbol{P}^{2}}\right)=\sqrt{\left(M_{\Sigma} c^{2}\right)^{2}+c^{2} \boldsymbol{P}^{2}}+\frac{1}{\sqrt{1+\frac{c^{2} \boldsymbol{P}^{2}}{\left(M_{\Sigma} c^{2}\right)^{2}}}} \sum_{a}\left(\frac{\boldsymbol{P}_{a}^{* 2}}{2 m_{a}}\right) \\
& =>H_{\{a\} \text { Newtonian }}\left(\left\{\boldsymbol{r}_{\boldsymbol{a}}^{*}\right\},\left\{\boldsymbol{P}_{\boldsymbol{a}}^{*}\right\}, \boldsymbol{R}_{\mathbf{C}}, \boldsymbol{P}\right) \approx \sqrt{\left(M_{\Sigma} c^{2}\right)^{2}+c^{2} \boldsymbol{P}^{2}}+\frac{1}{\sqrt{1+\frac{c^{2} \boldsymbol{P}^{2}}{\left(M_{\Sigma} c^{2}\right)^{2}}}} \sum_{a}\left(\frac{\boldsymbol{P}_{\boldsymbol{a}}{ }^{* 2}}{2 m_{a}}\right) \\
& \text { with } M_{\Sigma} \equiv \sum_{a} m_{a}
\end{aligned}
$$

We have also

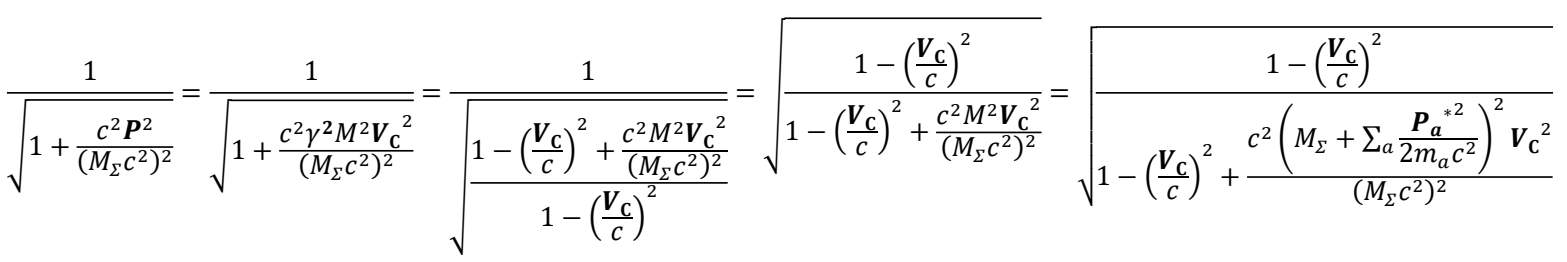

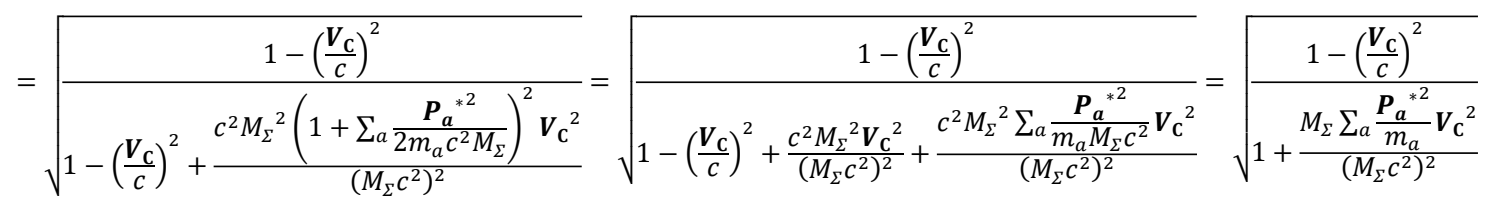

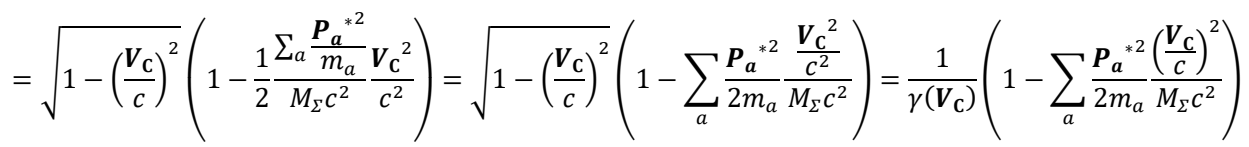

$$
\begin{aligned}
& =>H_{\{a\} \text { Newtonian }}\left(\left\{\boldsymbol{r}_{\boldsymbol{a}}^{*}\right\},\left\{\boldsymbol{P}_{\boldsymbol{a}}^{*}\right\}, \boldsymbol{R}_{\mathbf{C}}, \boldsymbol{P}\right) \\
& =\sqrt{\left(M_{\Sigma} c^{2}\right)^{2}+c^{2} \boldsymbol{P}^{2}}+\frac{1}{\gamma\left(\boldsymbol{V}_{\mathbf{C}}\right)}\left(1-\sum_{a} \frac{\boldsymbol{P}_{\boldsymbol{a}}{ }^{2}}{2 m_{a}} \frac{\left(\frac{\boldsymbol{V}_{\mathbf{C}}}{c}\right)^{2}}{M_{\Sigma} c^{2}}\right) \sum_{a}\left(\frac{\boldsymbol{P}_{\boldsymbol{a}}{ }^{2}}{2 m_{a}}\right) \\
& \approx \sqrt{\left(M_{\Sigma} c^{2}\right)^{2}+c^{2} \boldsymbol{P}^{2}}+\frac{1}{\gamma\left(\boldsymbol{V}_{\mathbf{C}}\right)} \sum_{a}\left(\frac{\boldsymbol{P}_{\boldsymbol{a}}{ }^{2}}{2 m_{a}}\right)
\end{aligned}
$$




$$
=>H_{\{a\} \text { Newtonian }}\left(\left\{\boldsymbol{r}_{\boldsymbol{a}}^{*}\right\},\left\{\boldsymbol{P}_{\boldsymbol{a}}{ }^{*}\right\}, \boldsymbol{R}_{\mathbf{C}}, \boldsymbol{P}\right) \approx \sqrt{\left(M_{\Sigma} c^{2}\right)^{2}+c^{2} \boldsymbol{P}^{2}}+\frac{1}{\gamma\left(\boldsymbol{V}_{\mathbf{C}}\right)} \sum_{a}\left(\frac{\boldsymbol{P}_{\boldsymbol{a}}{ }^{* 2}}{2 m_{a}}\right)
$$

This result is surprisingly for the second term $\frac{1}{\gamma\left(V_{\mathbf{C}}\right)} \sum_{a}\left(\frac{\boldsymbol{P}_{a}^{* 2}}{2 m_{a}}\right)$ because the dilation of time factor $\gamma\left(\boldsymbol{V}_{\mathbf{C}}\right)$ divides the internal ("kinematic") energy instead of multiplying it as in the relation $\mathrm{E}=\gamma \mathrm{E}^{*}$.

$$
\begin{aligned}
& \text { - } H_{\text {Newtonian }}\left(\left\{\boldsymbol{r}_{\boldsymbol{a}}^{*}\right\},\left\{\boldsymbol{P}_{\boldsymbol{a}}{ }^{*}\right\}, \boldsymbol{R}_{\mathbf{C}}, \boldsymbol{P}\right) \approx\left(M_{\Sigma} c^{2}\right)\left[1+\frac{1}{2} \frac{c^{2}}{\left(M_{\Sigma} c^{2}\right)^{2}}\left(\sum_{a}\left(\frac{M_{\Sigma}}{m_{a}}\right) \boldsymbol{P}_{\boldsymbol{a}}{ }^{* 2}+\boldsymbol{P}^{2}\right)\right] \\
& =M_{\Sigma} c^{2}+\frac{1}{2} \frac{1}{M_{\Sigma}}\left(\sum_{a}\left(\frac{M_{\Sigma}}{m_{a}}\right) \boldsymbol{P}_{\boldsymbol{a}}{ }^{2}+\boldsymbol{P}^{2}\right)=M_{\Sigma} c^{2}+\sum_{a}\left(\frac{\boldsymbol{P}_{\boldsymbol{a}}{ }^{2}}{2 m_{a}}\right)+\frac{\boldsymbol{P}^{2}}{2 M_{\Sigma}} \\
& =>H_{\text {Newtonian }}\left(\left\{\boldsymbol{r}_{\boldsymbol{a}}^{*}\right\},\left\{\boldsymbol{P}_{\boldsymbol{a}}{ }^{*}\right\}, \boldsymbol{R}_{\mathbf{C}}, \boldsymbol{P}\right) \approx M_{\Sigma} c^{2}+\sum_{a} \frac{\boldsymbol{P}_{\boldsymbol{a}}{ }^{* 2}}{2 m_{a}}+\frac{\boldsymbol{P}^{2}}{2 M_{\Sigma}} \\
& =\sqrt{\left[\sum_{a} \sqrt{\left(m_{a} c^{2}\right)^{2}+c^{2} \boldsymbol{P}_{\boldsymbol{a}}^{* 2}}\right]^{2}+c^{2} \boldsymbol{P}^{2}} \\
& =\left[\sum_{a} \sqrt{\left(m_{a} c^{2}\right)^{2}+c^{2} \boldsymbol{P}_{\boldsymbol{a}}{ }^{* 2}}\right] \sqrt{1+\frac{c^{2} \boldsymbol{P}^{2}}{\left[\sum_{a} \sqrt{\left(m_{a} c^{2}\right)^{2}+c^{2} \boldsymbol{P}_{\boldsymbol{a}}{ }^{2}}\right]^{2}}} \\
& =\left[\sum_{a} \sqrt{\left(m_{a} c^{2}\right)^{2}+c^{2} \boldsymbol{P}_{\boldsymbol{a}}{ }^{2}}\right]\left(1+\frac{1}{2} \frac{c^{2} \boldsymbol{P}^{2}}{\left[\sum_{a} \sqrt{\left(m_{a} c^{2}\right)^{2}+c^{2} \boldsymbol{P}_{\boldsymbol{a}}{ }^{2}}\right]^{2}}\right) \\
& =>H_{C, \text { Newtonian }}\left(\left\{\boldsymbol{r}_{\boldsymbol{a}}^{*}\right\},\left\{\boldsymbol{P}_{\boldsymbol{a}}{ }^{*}\right\}, \boldsymbol{R}_{\mathbf{C}}, \boldsymbol{P}\right) \approx \sum_{a} \sqrt{\left(m_{a} c^{2}\right)^{2}+c^{2} \boldsymbol{P}_{\boldsymbol{a}}{ }^{* 2}}+\frac{1}{2} \frac{c^{2} \boldsymbol{P}^{2}}{\sum_{a} \sqrt{\left(m_{a} c^{2}\right)^{2}+c^{2} \boldsymbol{P}_{\boldsymbol{a}}{ }^{* 2}}}
\end{aligned}
$$

\section{- Hamiltonian equations}

We can verify if the form of the Hamiltonian verifies the Hamilton equation:

With

$$
\begin{aligned}
\frac{\partial H}{\partial \boldsymbol{P}_{\boldsymbol{a} \prime}^{*}}\left(\left\{\boldsymbol{r}_{\boldsymbol{a}}^{*}\right\},\left\{\boldsymbol{P}_{\boldsymbol{a}}^{*}\right\}, \boldsymbol{R}_{\mathbf{C}}, \boldsymbol{P}\right)=\frac{\partial}{\partial \boldsymbol{P}_{\boldsymbol{a}}^{*}} \sqrt{H^{*}\left(\left\{\boldsymbol{r}_{\boldsymbol{a}}^{*}\right\},\left\{\boldsymbol{P}_{\boldsymbol{a}}^{*}\right\}\right)^{2}+c^{2} \boldsymbol{P}^{2}}= \\
=H^{*}\left(\left\{\boldsymbol{r}_{\boldsymbol{a}}^{*}\right\},\left\{\boldsymbol{P}_{\boldsymbol{a}}^{*}\right\}\right) \frac{\partial H^{*}\left(\left\{\boldsymbol{r}_{\boldsymbol{a}}^{*}\right\},\left\{\boldsymbol{P}_{\boldsymbol{a}}^{*}\right\}\right)}{\partial \boldsymbol{P}_{\boldsymbol{a} \prime}^{*}} \frac{1}{H\left(\left\{\boldsymbol{r}_{\boldsymbol{a}}^{*}\right\},\left\{\boldsymbol{P}_{\boldsymbol{a}}^{*}\right\}, \boldsymbol{R}_{\mathbf{C}}, \boldsymbol{P}\right)}
\end{aligned}
$$

$$
\begin{aligned}
\frac{\partial H^{*}\left(\left\{\boldsymbol{r}_{\boldsymbol{a}}^{*}\right\},\left\{\boldsymbol{P}_{\boldsymbol{a}}{ }^{*}\right\}\right)}{\partial \boldsymbol{P}_{\boldsymbol{a}^{\prime}}^{*}} & =\sum_{a} \frac{\partial}{\partial \boldsymbol{P}_{\boldsymbol{a}}^{*}} \sqrt{\left(m_{a} c^{2}\right)^{2}+c^{2} \boldsymbol{P}_{\boldsymbol{a}}{ }^{* 2}}=\sum_{a} c^{2} \boldsymbol{P}_{\boldsymbol{a}}{ }^{*} \frac{\partial \boldsymbol{P}_{\boldsymbol{a}}{ }^{*}}{\partial \boldsymbol{P}_{\boldsymbol{a}^{\prime}}^{*}} \frac{1}{H_{a}^{*}\left(\left\{\boldsymbol{r}_{\boldsymbol{a}}^{*}\right\},\left\{\boldsymbol{P}_{\boldsymbol{a}}{ }^{*}\right\}\right)} \\
= & \sum_{a} c^{2} \delta_{a a^{\prime}} \frac{\boldsymbol{P}_{\boldsymbol{a}}{ }^{*}}{\sqrt{\left(m_{a} c^{2}\right)^{2}+c^{2} \boldsymbol{P}_{\boldsymbol{a}}{ }^{* 2}}}=\frac{c^{2} \boldsymbol{P}_{\boldsymbol{a}^{\prime}}{ }^{*}}{\sqrt{\left.\left(m_{\boldsymbol{a}}{ }^{2}\right)^{2}\right)^{2}+c^{2} \boldsymbol{P}_{\boldsymbol{a}^{\prime}}{ }^{* 2}}}=\frac{c^{2} \boldsymbol{P}_{\boldsymbol{a}}{ }^{*}}{H_{a^{\prime}}^{*}\left(\boldsymbol{r}_{\boldsymbol{a}^{\prime}}^{*} \boldsymbol{P}_{\boldsymbol{a}^{\prime}}{ }^{*}\right)}
\end{aligned}
$$

Then 


$$
\begin{aligned}
& \frac{\partial H}{\partial \boldsymbol{P}_{\boldsymbol{a}}^{*}}\left(\left\{\boldsymbol{r}_{\boldsymbol{a}}^{*}\right\},\left\{\boldsymbol{P}_{\boldsymbol{a}}^{*}\right\}, \boldsymbol{R}_{\mathbf{C}}, \boldsymbol{P}\right)= \frac{c^{2} \boldsymbol{P}_{\boldsymbol{a}}^{*}}{H_{a}^{*}\left(\boldsymbol{r}_{\boldsymbol{a}}^{*}, \boldsymbol{P}_{\boldsymbol{a}}{ }^{*}\right)} \frac{H^{*}\left(\left\{\boldsymbol{r}_{\boldsymbol{a}}^{*}\right\},\left\{\boldsymbol{P}_{\boldsymbol{a}}{ }^{*}\right\}\right)}{H\left(\left\{\boldsymbol{r}_{\boldsymbol{a}}^{*}\right\},\left\{\boldsymbol{P}_{\boldsymbol{a}}{ }^{*}\right\}, \boldsymbol{R}_{\mathbf{C}}, \boldsymbol{P}\right)} \\
&= \frac{c^{2} \boldsymbol{P}_{\boldsymbol{a}}^{*} \cdot H^{*}\left(\left\{\boldsymbol{r}_{\boldsymbol{a}}^{*}\right\},\left\{\boldsymbol{P}_{\boldsymbol{a}}{ }^{*}\right\}\right)}{H\left(\left\{\boldsymbol{r}_{\boldsymbol{a}}^{*}\right\},\left\{\boldsymbol{P}_{\boldsymbol{a}}^{*}\right\}, \boldsymbol{R}_{\mathbf{C}}, \boldsymbol{P}\right) \cdot H_{a}^{*}\left(\boldsymbol{r}_{\boldsymbol{a}}^{*}, \boldsymbol{P}_{\boldsymbol{a}}{ }^{*}\right)} \\
&=>c^{2} \frac{\boldsymbol{P}_{\boldsymbol{a}}^{*}}{\gamma \cdot E_{a}^{*}}=\frac{\partial H}{\partial \boldsymbol{P}_{\boldsymbol{a}}^{*}}\left(\left\{\boldsymbol{r}_{\boldsymbol{a}}^{*}\right\},\left\{\boldsymbol{P}_{\boldsymbol{a}}^{*}\right\}, \boldsymbol{R}_{\mathbf{C}}, \boldsymbol{P}\right)
\end{aligned}
$$

But as for the center of mass we can write

$\boldsymbol{P}_{\boldsymbol{a}}^{*}=\frac{E_{a}^{*}}{c^{2}} \frac{d \boldsymbol{r}_{a}^{*}}{d t^{*}}$ with $E_{a}^{*}=\gamma_{a}^{*} E_{a}^{K_{a}^{*}}$

Where $E_{a}^{K_{a}^{*}}$ is the internal energy of the particle "a" in its own center of mass. This internal energy is equal to its mass only when the particle is free (as for the global center of mass).

$$
\left(E_{a}^{K_{a}^{*}}\right)_{a \text { is free }}=m_{a} c^{2}
$$

Then we can write in general

$$
\begin{gathered}
c^{2} \frac{\frac{E_{a}^{*}}{c^{2}} \frac{d \boldsymbol{r}_{\boldsymbol{a}}^{*}}{d t^{*}}}{\gamma \cdot E_{a}^{*}}=\frac{\partial H}{\partial \boldsymbol{P}_{\boldsymbol{a}}^{*}}\left(\left\{\boldsymbol{r}_{\boldsymbol{a}}^{*}\right\},\left\{\boldsymbol{P}_{\boldsymbol{a}}{ }^{*}\right\}, \boldsymbol{R}_{\mathbf{C}}, \boldsymbol{P}\right) \\
\frac{1}{\gamma} \frac{d \boldsymbol{r}_{\boldsymbol{a}}^{*}}{d t^{*}}=\frac{d \boldsymbol{r}_{\boldsymbol{a}}^{*}}{d t}=\frac{\partial H}{\partial \boldsymbol{P}_{\boldsymbol{a}}^{*}}\left(\left\{\boldsymbol{r}_{\boldsymbol{a}}^{*}\right\},\left\{\boldsymbol{P}_{\boldsymbol{a}}^{*}\right\}, \boldsymbol{R}_{\mathbf{C}}, \boldsymbol{P}\right) \\
=>\frac{d \boldsymbol{r}_{\boldsymbol{a}}^{*}}{d t}=\frac{\partial H}{\partial \boldsymbol{P}_{\boldsymbol{a}}^{*}}\left(\left\{\boldsymbol{r}_{\boldsymbol{a}}^{*}\right\},\left\{\boldsymbol{P}_{\boldsymbol{a}}^{*}\right\}, \boldsymbol{R}_{\mathbf{C}}, \boldsymbol{P}\right)
\end{gathered}
$$

This is again coherent with a first Hamiltonian equation.

$$
\begin{aligned}
& \frac{\partial H}{\partial \boldsymbol{r}_{\boldsymbol{a}}^{*}}\left(\left\{\boldsymbol{r}_{\boldsymbol{a}}^{*}\right\},\left\{\boldsymbol{P}_{\boldsymbol{a}}^{*}\right\}, \boldsymbol{R}_{\mathbf{C}}, \boldsymbol{P}\right)=\frac{\partial}{\partial \boldsymbol{r}_{\boldsymbol{a}}^{*}} \sqrt{H^{*}\left(\left\{\boldsymbol{r}_{\boldsymbol{a}}^{*}\right\},\left\{\boldsymbol{P}_{\boldsymbol{a}}^{*}\right\}\right)^{2}+c^{2} \boldsymbol{P}^{2}}= \\
& =\frac{H^{*}\left(\left\{\boldsymbol{r}_{\boldsymbol{a}}^{*}\right\},\left\{\boldsymbol{P}_{\boldsymbol{a}}^{*}\right\}\right)}{H\left(\left\{\boldsymbol{r}_{\boldsymbol{a}}^{*}\right\},\left\{\boldsymbol{P}_{\boldsymbol{a}}^{*}\right\}, \boldsymbol{R}_{\mathbf{C}}, \boldsymbol{P}\right)} \frac{\partial H^{*}\left(\left\{\boldsymbol{r}_{\boldsymbol{a}}^{*}\right\},\left\{\boldsymbol{P}_{\boldsymbol{a}}^{*}\right\}\right)}{\partial \boldsymbol{r}_{\boldsymbol{a} \prime}^{*}}=-\frac{H^{*}\left(\left\{\boldsymbol{r}_{\boldsymbol{a}}^{*}\right\},\left\{\boldsymbol{P}_{\boldsymbol{a}}^{*}\right\}\right)}{H\left(\left\{\boldsymbol{r}_{\boldsymbol{a}}^{*}\right\},\left\{\boldsymbol{P}_{\boldsymbol{a}}^{*}\right\}, \boldsymbol{R}_{\mathbf{C}}, \boldsymbol{P}\right)} \frac{d \boldsymbol{P}_{\boldsymbol{a}}{ }^{*}}{d t^{*}} \\
& \text { Since } \frac{\partial H^{*}\left(\left\{\boldsymbol{r}_{\boldsymbol{a}}^{*}\right\},\left\{\boldsymbol{P}_{\boldsymbol{a}}^{*}\right\}\right)}{\partial \boldsymbol{r}_{\boldsymbol{a}^{\prime}}}=\frac{\partial\left[\sum_{\boldsymbol{a}} \boldsymbol{P}_{\boldsymbol{a}}{ }^{*} \frac{d \boldsymbol{r}_{\boldsymbol{a}}^{*}}{d t}-L^{*}\right]}{\partial \boldsymbol{r}_{\boldsymbol{a}^{\prime}}^{*}}=-\frac{\partial L^{*}}{\partial \boldsymbol{r}_{\boldsymbol{a}^{\prime}}^{*}}=-\frac{d \boldsymbol{P}_{\boldsymbol{a}}{ }^{*}}{d t^{*}} \\
& \frac{\partial H}{\partial \boldsymbol{r}_{\boldsymbol{a}}^{*}}\left(\left\{\boldsymbol{r}_{\boldsymbol{a}}^{*}\right\},\left\{\boldsymbol{P}_{\boldsymbol{a}}{ }^{*}\right\}, \boldsymbol{R}_{\mathbf{C}}, \boldsymbol{P}\right)=-\frac{E^{*}}{\gamma E^{*}} \frac{d \boldsymbol{P}_{\boldsymbol{a}}{ }^{*}}{d t^{*}}=-\frac{1}{\gamma} \frac{d \boldsymbol{P}_{\boldsymbol{a}}{ }^{*}}{d t^{*}}=-\frac{d \boldsymbol{P}_{\boldsymbol{a}}{ }^{*}}{d t} \\
& =>\frac{d \boldsymbol{P}_{\boldsymbol{a}}^{*}}{d t}=-\frac{\partial H}{\partial \boldsymbol{r}_{\boldsymbol{a}}^{*}}\left(\left\{\boldsymbol{r}_{\boldsymbol{a}}^{*}\right\},\left\{\boldsymbol{P}_{\boldsymbol{a}}^{*}\right\}, \boldsymbol{R}_{\mathbf{C}}, \boldsymbol{P}\right)
\end{aligned}
$$

This is again consistent with a second Hamiltonian equation.

We then see that for the particular variables chosen in the Lagrangian analysis $\left(\frac{d r_{a}^{*}}{d t}, \boldsymbol{r}_{a}^{*}\right)$ we find what we should expect for a Hamiltonian analysis with the variable $\left(\boldsymbol{P}_{\boldsymbol{a}}{ }^{*}, \boldsymbol{r}_{\boldsymbol{a}}^{*}\right)$, that is to say the Hamiltonian equation.

For the center of mass we have obviously

$$
\begin{gathered}
\frac{\partial H}{\partial \boldsymbol{P}}\left(\left\{\boldsymbol{r}_{\boldsymbol{a}}^{*}\right\},\left\{\boldsymbol{P}_{\boldsymbol{a}}^{*}\right\}, \boldsymbol{R}_{\mathbf{C}}, \boldsymbol{P}\right)=\frac{\partial}{\partial \boldsymbol{P}} \sqrt{H^{*}\left(\left\{\boldsymbol{r}_{\boldsymbol{a}}^{*}\right\},\left\{\boldsymbol{P}_{\boldsymbol{a}}^{*}\right\}\right)^{2}+c^{2} \boldsymbol{P}^{2}}=\frac{c^{2} \boldsymbol{P}}{H\left(\left\{\boldsymbol{r}_{\boldsymbol{a}}^{*}\right\},\left\{\boldsymbol{P}_{\boldsymbol{a}}^{*}\right\}, \boldsymbol{R}_{\mathbf{C}}, \boldsymbol{P}\right)} \\
=\frac{c^{2} \frac{E}{c^{2}} \boldsymbol{V}_{\mathbf{C}}}{E}=\boldsymbol{V}_{\mathbf{C}}
\end{gathered}
$$




$$
=>\boldsymbol{V}_{\mathbf{C}}=\frac{\partial H}{\partial \boldsymbol{P}}\left(\left\{\boldsymbol{r}_{\boldsymbol{a}}^{*}\right\},\left\{\boldsymbol{P}_{\boldsymbol{a}}^{*}\right\}, \boldsymbol{R}_{\mathbf{C}}, \boldsymbol{P}\right)
$$

The second equation, as already showed (cf. [2]):

$$
\frac{d \boldsymbol{P}}{d t}=\frac{\partial \sum_{\boldsymbol{a}} \boldsymbol{P}_{\boldsymbol{a}}{ }^{*} \frac{d \boldsymbol{r}_{\boldsymbol{a}}^{*}}{d t}+\boldsymbol{P} \boldsymbol{V}_{\mathbf{C}}-H^{\prime}}{\partial \boldsymbol{R}_{\mathbf{C}}}=-\frac{\partial H^{\prime}}{\partial \boldsymbol{R}_{\mathbf{C}}}\left(\left\{\boldsymbol{r}_{\boldsymbol{a}}^{*}\right\},\left\{\boldsymbol{P}_{\boldsymbol{a}}^{*}\right\}, \boldsymbol{R}_{\mathbf{C}}, \boldsymbol{P}\right)
$$

We see that if we want to quantize any system in parallel with its center of mass, we should choose the quantum operator associated to the corresponding canonical couples of classical variables:

- $\left\{\left(\boldsymbol{r}_{\boldsymbol{a}}^{*}, \boldsymbol{P}_{\boldsymbol{a}}{ }^{*}\right)\right\},\left(\boldsymbol{R}_{\mathbf{C}}, \boldsymbol{P}\right)$ for a system of particles

- $\left\{\left(\varphi^{*}, \frac{\partial \varphi^{*}}{\partial \boldsymbol{r}^{*}}\right)\right\},\left(\boldsymbol{R}_{\mathbf{C}}, \boldsymbol{P}\right)$ for a field (scalar for example)

- etc.. 


\subsection{Application: the electromagnetic model of the electron [3], [9 ], [23]-[25]}

Just before and during the construction of the Special Relativity, some theoretical physicists used an electromagnetic model of the electron in order to untangle the ball of wool constituted by different to date of physical theories and experiments about the electrodynamics (and the optic) of moving bodies. The model of electron was used by notably Lorentz and improving by Poincaré (who shares with Einstein the privilege to have realized the last step of the discovery/invention of Special Relativity, both (very different) way of thinking have their own charm) which is interesting to use at least to treat classically the interaction between matter and electromagnetic field without divergence. The latter appears indeed for a material point as showed in [1]. One can always (in a classical universe with matter and electromagnetism living in a static Minkowskian space-time) physically replace a material point by a continuum if one always works for dimensions infinitely larger than the dimension of the continuum. The interest is to have a clear mathematic expression for the mass, even if the model is actually fundamentally wrong (but the ugly last point is here "sufficiently" hidden).

My interest in using this model is to see how a complex system behaves with the particular choice of variables and so thus to see the influence of the dynamics of the center of mass on the internal dynamics, in particular the mass behaviour itself. The model I decided to use is slightly different from the one used by Poincare since I want to maintain the mass of the continuum without let all the mass to the electromagnetic energy field (as Poincaré \& Lorentz \& others have done).

I will present the first attempt of the electron model which is unstable and then the one used by Poincaré with his internal "pressure".

The electron model is:

- a continuum spherical surface in its rest frame $K^{*}$ characterized by a surface density of mass $\sigma$;

0 the speed of all the material points of the continuum are radial (at an instant $t$ )

$\circ$ and the mass distribution is spherical $\mathrm{K}^{*}$ (at an instant $\mathrm{t}$ ).

We assume that, the internal spherical behaviour is maintained during motion, although according to [9], this model is in fact unstable.

The Lagrangian is

$$
\begin{aligned}
& L\left(\left\{\boldsymbol{r}_{\boldsymbol{a}}(t)\right\},\left\{\frac{d \boldsymbol{r}_{\boldsymbol{a}}}{d t}\right\}, t\right)=\sum_{a}\left[-m_{a} \cdot c \frac{d s_{a}}{d t}-\frac{e_{a}}{c} \cdot A^{i}\left(x_{a i}\right) \frac{d x_{a i}}{d t}\right] \\
& =>L^{\prime}\left(\left\{\boldsymbol{r}_{\boldsymbol{a}}^{*}(t)\right\},\left\{\frac{d \boldsymbol{r}_{a}^{*}}{d t}\right\}, \boldsymbol{R}_{\mathbf{C}}, \boldsymbol{V}_{\mathbf{C}}, t\right)=-\frac{\sum a\left[\frac{m_{a} \cdot c^{2}}{\gamma a^{*}}+e_{a} \cdot \varphi^{*}\left(\boldsymbol{r}_{\boldsymbol{a}}^{*}, t^{*}\right)\right]}{\gamma\left(\boldsymbol{V}_{\mathbf{C}}\right)}+\sum_{a} \frac{e_{a}}{c} \boldsymbol{A}^{*}\left(\boldsymbol{r}_{\boldsymbol{a}}^{*}, t^{*}\right) \cdot \frac{d \boldsymbol{r}_{a}^{*}}{d t} \\
& =-\frac{\sum a\left[\frac{m_{a} \cdot c^{2}}{\gamma a^{*}}+e_{a} \cdot \varphi^{*}\left(r_{\boldsymbol{a}}^{*}, t^{*}\right)\right]}{\gamma\left(\boldsymbol{V}_{\mathbf{C}}\right)} \text { via the isotropy hypothesis } \\
& =-\frac{\iint\left[\frac{\sigma_{m}^{*} \cdot c^{2}}{\gamma^{*}}+\sigma_{e}^{*} \cdot \varphi^{*}\left(\boldsymbol{r}^{*}, t^{*}\right)\right] d s^{*}}{\gamma\left(\boldsymbol{V}_{\mathbf{C}}\right)} \text { since we have a continuum }
\end{aligned}
$$


$=-\frac{\left[\frac{\sigma_{m}^{*} \cdot c^{2}}{\gamma^{*}}+\sigma_{e}^{*} \cdot \varphi^{*}\left(\boldsymbol{r}^{*}, t^{*}\right)\right] S^{*}}{\gamma\left(\boldsymbol{V}_{\mathbf{C}}\right)}$ via the isotropy of the speed in $\mathrm{K}^{*}$

$=-\frac{\frac{M_{\Sigma} \cdot c^{2}}{\gamma^{*}}+e \cdot \varphi^{*}\left(r^{*}, t^{*}\right)}{\gamma\left(V_{\mathbf{C}}\right)}$ since the additive mass $M_{\Sigma}=\sigma_{m}^{*} S^{*}$ and the charge $\mathrm{e}=\sigma_{e}^{*} S^{*}$ are relativistic invariants and the distribution of material points is spherical.

We have $e . \varphi^{*}\left(r^{*}, t^{*}\right)=E_{e m}^{*}\left(r^{*}\right)=E_{e m, e q}^{*} \frac{r_{e q}^{*}}{r^{*}}$ where $E_{e m}^{*}\left(r^{*}\right)$ is the electromagnetic energy and the quantities with index "eq" are the associated quantities for an eventual equilibrium point.

We have also $\gamma^{*}=\gamma^{*}\left(\frac{d r^{*}}{d t^{*}}\right)=\frac{1}{\sqrt{1-\frac{1}{c^{2}}\left(\frac{d r^{*}}{d t^{*}}\right)^{2}}}=\gamma^{*}\left(\gamma\left(\boldsymbol{V}_{\mathbf{C}}\right) \frac{d r^{*}}{d t}\right)=\frac{1}{\sqrt{1-\frac{\gamma\left(V_{\mathbf{C}}\right)^{2}}{c^{2}}\left(\frac{d r^{*}}{d t}\right)^{2}}}=\gamma^{*}\left(\boldsymbol{V}_{\mathbf{C}}, \frac{d r^{*}}{d t}\right)$

$=>L^{\prime}\left(\left\{\boldsymbol{r}_{\boldsymbol{a}}^{*}(t)\right\},\left\{\frac{d \boldsymbol{r}_{a}^{*}}{d t}\right\}, \boldsymbol{R}_{\mathbf{C}}, \boldsymbol{V}_{\mathbf{C}}, t\right)=L^{\prime}\left(r^{*}, \frac{d r^{*}}{d t}, \boldsymbol{R}_{\mathbf{C}}, \boldsymbol{V}_{\mathbf{C}}, t\right)=-\frac{1}{\gamma\left(\boldsymbol{V}_{\mathbf{C}}\right)}\left(\frac{M_{\Sigma} c^{2}}{\gamma^{*}\left(\boldsymbol{V}_{\mathbf{C}}, \frac{d r^{*}}{d t}\right)}+E_{e m, e q}^{*} \frac{r_{e q}^{*}}{r^{*}}\right)$

with

$\boldsymbol{P}_{\boldsymbol{c}}=\frac{\partial L^{\prime}}{\partial \boldsymbol{v}_{\mathrm{C}}}=\gamma\left(\boldsymbol{v}_{\mathrm{C}}\right)\left(\frac{\sum_{a}\left[\gamma_{a}{ }^{*} \cdot m_{a} \cdot c^{2}+e_{a} \cdot \varphi^{*}\left(\boldsymbol{r}_{\boldsymbol{a}}^{*}, t^{*}\right)\right]}{c^{2}}\right) \boldsymbol{v}_{\mathrm{C}}=\gamma\left(\boldsymbol{v}_{\mathrm{C}}\right)\left(\gamma^{*} \cdot M_{\Sigma}+\frac{E_{e m, e q}^{*}}{c^{2}} \frac{r_{e q}^{*}}{r^{*}}\right) \boldsymbol{v}_{\mathrm{C}}$

$=>\boldsymbol{P}_{\boldsymbol{c}}=\gamma\left(\boldsymbol{V}_{\mathrm{C}}\right) M \boldsymbol{v}_{\mathrm{C}}$

With $M=\gamma^{*} \cdot M_{\Sigma}+\frac{E_{e m, e q}^{*}}{c^{2}} \frac{r_{e q}^{*}}{r^{*}}$

And $\gamma^{*}=\gamma^{*}\left(\boldsymbol{V}_{\mathbf{C}}, \frac{d r^{*}}{d t}\right)=\frac{1}{1-\frac{\gamma\left(V_{\mathbf{C}}\right)^{2}}{c^{2}}\left(\frac{d r^{*}}{d t}\right)^{2}}$

We see that the mass is $\left(\right.$ modulo $c^{2}$ ) the sum of the total internal free energy $\gamma^{*} . M_{\Sigma}$ with the electromagnetic energy $\frac{E_{e m, e q}^{*}}{c^{2}} \frac{r_{e q}^{*}}{r^{*}}$ (a potential energy).

Moreover, the value of the mass depends of the "external" dynamics of the center of mass.

The relativistic dynamic is:

$$
\begin{gathered}
\frac{d}{d t}\left(\gamma\left(\boldsymbol{V}_{\mathbf{C}}\right) \frac{E^{*}}{c^{2}} \boldsymbol{V}_{\mathbf{C}}\right)=\frac{\partial}{\partial \boldsymbol{R}_{\mathbf{C}}} L^{\prime}\left(r^{*}, \frac{d r^{*}}{d t}, \boldsymbol{R}_{\mathbf{C}}, \boldsymbol{V}_{\mathbf{C}}, t\right) \\
\frac{d}{d t}\left(\gamma_{a}^{*} m_{a} \cdot \frac{d \boldsymbol{r}_{\boldsymbol{a}}^{*}}{d t^{*}}\right)=\frac{1}{\gamma\left(\boldsymbol{V}_{\mathbf{C}}\right)} \frac{\partial}{\partial \boldsymbol{r}_{\boldsymbol{a}}^{*}} L^{*}\left(\left\{\boldsymbol{r}_{\boldsymbol{a}}^{*}\right\},\left\{\gamma\left(\boldsymbol{V}_{\mathbf{C}}\right) \frac{d \boldsymbol{r}_{\boldsymbol{a}}^{*}}{d t}\right\}\right) \\
=>\frac{d}{d t}\left(\gamma^{*} M_{\Sigma} \cdot \frac{d r^{*}}{d t^{*}}\right)=\frac{-1}{\gamma\left(\boldsymbol{v}_{\mathbf{C}}\right)} \frac{\partial}{\partial r^{*}}\left(\gamma^{*}\left(\boldsymbol{V}_{\mathbf{C}} \frac{d r^{*}}{d t}\right) \cdot M_{\Sigma} c^{2}+E_{e m, e q}^{*} \frac{r_{e q}^{*}}{r^{*}}\right)=\frac{1}{\gamma\left(\boldsymbol{v}_{\mathbf{C}}\right)} E_{e m, e q}^{*} \frac{r_{e q}^{*}}{r^{* 2}} \\
=>\frac{d}{d t}\left(\gamma\left(\boldsymbol{V}_{\mathbf{C}}\right) \cdot \gamma^{*} M_{\Sigma} \cdot \frac{d r^{*}}{d t}\right)=\frac{1}{\gamma\left(\boldsymbol{v}_{\mathrm{C}}\right)} E_{e m, e q}^{*} \frac{r_{e q}^{*}}{r^{* 2}}
\end{gathered}
$$

One can see that this model is internally radially unstable since there is only a repulsive term. 
In order to improve he model we can add to it a truncated cosmological constant [9] which is null everywhere but not into the spherical electron.

The new Lagrangian is ([9] \& [3])

$$
L^{\prime}\left(r^{*}, \frac{d r^{*}}{d t}, \boldsymbol{R}_{\mathbf{C}}, \boldsymbol{V}_{\mathbf{C}}, t\right)=-\frac{1}{\gamma\left(\boldsymbol{V}_{\mathbf{C}}\right)}\left(\frac{M_{\Sigma} c^{2}}{\gamma^{*}\left(\boldsymbol{V}_{\mathbf{C}}, \frac{d r^{*}}{d t}\right)}+E_{e m, e q}^{*} \frac{r_{e q}^{*}}{r^{*}}-\frac{c^{4}}{8 \pi k} \iiint \Lambda_{P} \cdot \theta\left(R^{*}-r^{*}\right) \sqrt{-g^{*}} d^{3} \boldsymbol{R}^{*}\right)
$$

With

$$
\begin{aligned}
\theta\left(R^{*}-r^{*}\right) & \equiv 1 \text { for } R^{*} \leq r^{*} \\
& \equiv 0 \operatorname{for}^{*}>r^{*}
\end{aligned}
$$

But the space-time is Minkowskian and the electron is spherical in $\mathrm{K}^{*}$. Then

$$
L^{\prime}\left(r^{*}, \frac{d r^{*}}{d t}, \boldsymbol{R}_{\mathbf{C}}, \boldsymbol{V}_{\mathbf{C}}, t\right)=-\frac{1}{\gamma\left(\boldsymbol{V}_{\mathbf{C}}\right)}\left(\frac{M_{\Sigma} c^{2}}{\gamma^{*}\left(\boldsymbol{V}_{\mathbf{C}}, \frac{d r^{*}}{d t}\right)}+E_{e m, e q}^{*} \frac{r_{e q}^{*}}{r^{*}}-\frac{c^{4}}{8 \pi k} \frac{4}{3} \pi \Lambda_{P} \cdot r^{* 3}\right)
$$

\section{A false problem}

We can remark that this Lagrangian naively suggests that the interaction terms acts instantaneously which would be inconsistent with Relativity. But actually the interaction terms come from fields that acts just exactly at the points where the material points are located, that is to say on the sphere and not at the center of the sphere.

\section{A digression towards some intriguing Uniform-Energy-Region}

The cosmological term in the Lagrangian is not the one used by Einstein since it is not applied to the whole space-time. This is very surprising for me since the general famous theorem (Lovelock) established that the cosmological term à la Einstein (in addition to the Ricci term) is the only one allowed in General Relativity in order to respect the general requirement of this theory: second order equation for dynamics and invariance of physical laws for any transformation of coordinates. A natural question is why the addition of the Poincaré term is authorized in Relativity ? In a more intuitive reasoning (which allows to reveal the solution): saying that a cosmological term applied only to a given fixed region seems to contradict the epistemological views of General Relativity [22] saying in particular that any effect of a phenomenon has to be caused by a direct measurable cause. This direct measurable cause has to be a physical phenomenon, governed by dynamical equations, which interact with other fields and matters (that is why reference frame must not be allowed to influence phenomena via inertial forces, the equivalence principle permitting precisely to make the latter dynamic by unifying them with the dynamical field of gravity). The solution to my problem is therefore that the boundary of the region, where the Poincaré pressure term is applied, is dynamically coupled with the distribution of the material system localized in the region. This has an interesting consequence: General Relativity allows a priori the existence of an arbitrary number $\mathrm{n}$ of deformed closed surfaces surrounding internal regions, of volume $\iiint \theta\left(\left\|\boldsymbol{R}_{n}\right\|-\left\|\boldsymbol{r}_{n}\right\|\right) \sqrt{-g} \cdot d^{3} \boldsymbol{R}_{n}$, each containing a "Constant-cosmological" term $\Lambda_{n}$ with an arbitrary value. This in the condition that 
all these borders are dynamical coupled with a border variable $\boldsymbol{r}_{\boldsymbol{n}}$. Explicitly, General Relativity permits an action as

$$
\begin{array}{r}
S\left[\left\{g_{i k}(x, t)\right\},\left\{r_{n}(t)\right\}\right] \\
=\frac{-c^{3}}{16 \pi k} \iiint \int\left(R-2 \Lambda_{E}\right) \sqrt{-g} d \Omega+\sum_{n} \frac{c^{4}}{8 \pi k} \iiint \int \Lambda_{n} \cdot \theta\left(\left\|\boldsymbol{R}_{n}\right\|-\left\|\boldsymbol{r}_{n}\right\|\right) \sqrt{-g} \cdot d^{3} \boldsymbol{R}_{\boldsymbol{n}} d t+S\left[\left\{r_{n}(t), \ldots\right\}\right]
\end{array}
$$

Thus, Lovelock theorem applied, as it should, to a free gravitation field and the other "cosmological" terms are not affected by it since they necessitate the use of other dynamical variables.

Of course, although permit, the other cosmological terms are not very "natural" because we have to add them arbitrary by hand. However they are not more "unnatural" than the complex topologies already often used and a priori allowed. If one accepts such new terms we must therefore complete the action with another part implying the dynamic of a 2D membrane for which every point behaves as a material point, each providing a "ds" term in the action. Hence, this membrane is sensitive to (as it should) the gravitation field (and a priori only to it) and is deformed by it. We can imagine a space time bathed by these Uniform-Energy-Regions. The problem of this kind of Uniform-Energy-Regions is the instabilities of their shape since they behave internally like a dynamical min-de-sitter (or antide-sitter) universe and not like a wiser Einstein-static one. Another problem that comes in mind is the possible appearance of gravitational singularities when 2 free point of the same surface (or even several surface) meet at the same point during their "free" movement (but this problem can be maybe cured by a quantum "bandage"). In spite of all these oddities, it is important to keep in mind (surely already known, perhaps by Dirac) all the mathematical possibilities permited by the standard paradigm of physics, which is still today partly constituted by classical General Relativity. After a reading of the Jean Pierre Luminet's book [17], it seems that these speculations look a bit like the concept of gravastars which were conceptually invented in 2001 by Mazur \& Mottola: Is "the Uniform-Energy-Regions" the same speculative concept as gravastars ? Is the gravastar the rebirth of the old Lorentz-Poincaré electron in an astrophysical domain? One of the differences would be that the Uniform-Energy-Regions is put by hand as one can put by hand a cosmological constant or the existence of some material points instead of being the result of a dynamical collapse of an existing massive star.

\section{Returning to our initial problem}

$$
L^{\prime}\left(r^{*}, \frac{d r^{*}}{d t}, \boldsymbol{R}_{\mathbf{C}}, \boldsymbol{V}_{\mathbf{C}}, t\right)=-\frac{1}{\gamma\left(\boldsymbol{V}_{\mathbf{C}}\right)}\left(\frac{M_{\Sigma} c^{2}}{\gamma^{*}\left(\boldsymbol{V}_{\mathbf{C}}, \frac{d r^{*}}{d t}\right)}+E_{e m, e q}^{*} \frac{r_{e q}^{*}}{r^{*}}-\frac{c^{4}}{2 k} \frac{1}{3} \Lambda_{P} \cdot r^{* 3}\right)
$$

Which gives

$$
\begin{aligned}
\boldsymbol{P}_{\boldsymbol{c}}=\frac{\partial L^{\prime}}{\partial \boldsymbol{v}_{\mathbf{C}}}=\gamma\left(\boldsymbol{V}_{\mathbf{C}}\right) & \left(\frac{\sum_{a}\left[\gamma_{a}^{*} \cdot m_{a} \cdot c^{2}+e_{a} \cdot \varphi^{*}\left(\boldsymbol{r}_{\boldsymbol{a}}^{*}, t^{*}\right)\right]}{c^{2}}\right) \boldsymbol{v}_{\mathbf{C}} \\
= & \gamma\left(\boldsymbol{v}_{\mathbf{C}}\right)\left(\gamma^{*} \cdot M_{\Sigma}+\frac{E_{e m, e q}^{*}}{c^{2}} \frac{r_{e q}^{*}}{r^{*}}-\frac{c^{2}}{2 k} \frac{1}{3} \Lambda_{P} \cdot r^{* 3}\right) \boldsymbol{v}_{\mathbf{C}}
\end{aligned}
$$


$\Rightarrow>\boldsymbol{P}_{\boldsymbol{c}}=\gamma\left(\boldsymbol{V}_{\mathrm{C}}\right) M \boldsymbol{v}_{\mathrm{C}}$

With $M=\gamma^{*} \cdot M_{\Sigma}+\frac{E_{e m, e q}^{*}}{c^{2}} \frac{r_{e q}^{*}}{r^{*}}-\frac{c^{2}}{6 k} \Lambda_{P} \cdot r^{* 3}$

And $\gamma^{*}=\gamma^{*}\left(\boldsymbol{V}_{\mathbf{C}}, \frac{d r^{*}}{d t}\right)=\frac{1}{\sqrt{1-\frac{\gamma\left(V_{\mathbf{C}}\right)^{2}}{c^{2}}\left(\frac{d r^{*}}{d t}\right)^{2}}}$

We see that the mass is (modulo $c^{2}$ ) the sum of the total internal free energy with the electromagnetic energy (which behaves as a potential energy) and with the pressure-Poincaré energy.

Moreover, the value of the mass depends of the "external" dynamics of the center of mass.

The relativistic dynamic for the internal part is now:

$$
\begin{aligned}
& \frac{d}{d t}\left(\gamma_{a}^{*} m_{a} \cdot \frac{d \boldsymbol{r}_{\boldsymbol{a}}^{*}}{d t^{*}}\right)=\frac{1}{\gamma\left(\boldsymbol{V}_{\mathrm{C}}\right)} \frac{\partial}{\partial \boldsymbol{r}_{\boldsymbol{a}}^{*}} L^{*}\left(\left\{\boldsymbol{r}_{\boldsymbol{a}}^{*}\right\},\left\{\gamma\left(\boldsymbol{v}_{\mathbf{c}}\right) \frac{d \boldsymbol{r}_{\boldsymbol{a}}^{*}}{d t}\right\}\right) \\
& =>\frac{d}{d t}\left(\gamma\left(\boldsymbol{V}_{\mathrm{C}}\right) \cdot \gamma^{*} M_{\Sigma} \cdot \frac{d r^{*}}{d t}\right)=\frac{-1}{\gamma\left(v_{\mathrm{C}}\right)} \frac{\partial}{\partial r^{*}}\left(\gamma^{*}\left(\boldsymbol{V}_{\mathbf{C}}, \frac{d r^{*}}{d t}\right) \cdot M_{\Sigma} c^{2}+E_{e m, e q}^{*} \frac{r_{e q}^{*}}{r^{*}}-\frac{c^{4}}{6 k} \Lambda_{P} \cdot r^{* 3}\right) \\
& =\frac{1}{\gamma\left(V_{\mathrm{C}}\right)} E_{e m, e q}^{*} \frac{r_{e q}^{*}}{r^{* 2}}+\frac{1}{\gamma\left(V_{\mathrm{C}}\right)} \frac{c^{4}}{2 k} \Lambda_{P} \cdot r^{* 2} \\
& =>\frac{d}{d t}\left(\gamma\left(V_{\mathrm{C}}\right) \cdot \gamma^{*} M_{\Sigma} \cdot \frac{d r^{*}}{d t}\right)=\frac{1}{\gamma\left(v_{\mathrm{C}}\right)} E_{e m, e q}^{*} \frac{r_{e q}^{*}}{r^{* 2}}+\frac{1}{\gamma\left(v_{\mathrm{C}}\right)} \frac{c^{4}}{2 k} \Lambda_{P} \cdot r^{* 2}
\end{aligned}
$$

In addition to the repulsive Coulomb term, the Poincaré term add a pressure force

$$
\begin{aligned}
& S^{*} \cdot p=\frac{1}{\gamma\left(v_{\mathrm{c}}\right)} \frac{c^{4}}{2 k} \Lambda_{P} \cdot r^{* 2} \\
& \Rightarrow p=\frac{\frac{1 c^{4}}{\gamma\left(v_{\mathrm{c}}\right) 2 k} \Lambda_{P} \cdot r^{* 2}}{4 \pi r^{* 2}}=\frac{1}{\gamma\left(v_{\mathrm{c}}\right)} \frac{c^{4}}{8 \pi k} \Lambda_{P}
\end{aligned}
$$

In order to stabilize the sphere, we put $\Lambda_{P}=-\left|\Lambda_{P}\right|$

Hence we have

$$
\frac{d}{d t}\left(\gamma\left(\boldsymbol{V}_{\mathbf{C}}\right) \cdot \gamma^{*} M_{\Sigma} \cdot \frac{d r^{*}}{d t}\right)=\frac{1}{\gamma\left(\boldsymbol{V}_{C}\right)} E_{e m, e q}^{*} \frac{r_{e q}^{*}}{r^{* 2}}-\frac{1}{\gamma\left(\boldsymbol{V}_{C}\right)} \frac{c^{4}}{2 k}\left|\Lambda_{P}\right| \cdot r^{* 2}
$$

The internal equilibrium is realized when (we put by definition $r^{*}=r_{e q}^{*}$ ):

$$
\begin{gathered}
\left(\frac{1}{\gamma\left(\boldsymbol{V}_{\mathrm{C}}\right)} E_{e m, e q}^{*} \frac{r_{e q}^{*}}{r^{* 2}}=\frac{1}{\gamma\left(\boldsymbol{V}_{\mathrm{C}}\right)} \frac{c^{4}}{2 k}\left|\Lambda_{P}\right| \cdot r^{* 2}\right)_{r^{*}=r_{e q}^{*}} \\
<=>E_{e m, e q}^{*}=\frac{c^{4}}{2 k}\left|\Lambda_{P}\right| \cdot r_{e q}^{* 3} \\
<=>r_{e q}^{* 3}=2 \frac{k}{\left|\Lambda_{P}\right| c^{4}} E_{e m, e q}^{*}
\end{gathered}
$$


Moreover

$$
\begin{aligned}
& =>\frac{d}{d t}\left(\gamma\left(V_{\mathrm{C}}\right) \cdot \gamma^{*} M_{\Sigma} \cdot \frac{d r^{*}}{d t}\right)=\frac{1}{\gamma\left(v_{\mathrm{c}}\right)} E_{e m, e q}^{*} \frac{r_{e q}^{*}}{r^{* 2}}-\frac{1}{\gamma\left(v_{\mathrm{c}}\right)}\left(\frac{c^{4}}{2 k}\left|\Lambda_{P}\right|\right) \cdot r^{* 2}=\frac{1}{\gamma\left(v_{\mathrm{C}}\right)} E_{e m, e q}^{*} \frac{r_{e q}^{*}}{r^{* 2}}-\frac{1}{\gamma\left(v_{\mathrm{c}}\right)}\left(\frac{E_{e m, e q}^{*}}{r_{e q}^{*}}\right) \cdot r^{* 2} \\
& =>\frac{d}{d t}\left(\gamma\left(V_{\mathrm{C}}\right) \cdot \gamma^{*} M_{\Sigma} \cdot \frac{d r^{*}}{d t}\right)=\frac{1}{\gamma\left(V_{\mathrm{C}}\right)} E_{e m, e q}^{*}\left(\frac{r_{e q}^{*}}{r^{* 2}}-\frac{r^{* 2}}{r_{e q}^{* 3}}\right) \\
& M=\gamma^{*} \cdot M_{\Sigma}+\frac{E_{e m, e q}^{*}}{c^{2}} \frac{r_{e q}^{*}}{r^{*}}-\frac{c^{2}}{6 k} \Lambda_{P} \cdot r^{* 3}=\gamma^{*} \cdot M_{\Sigma}+\frac{E_{e m, e q}^{*}}{c^{2}} \frac{r_{e q}^{*}}{r^{*}}+\frac{1}{3}\left(\frac{c^{2}}{2 k}\left|\Lambda_{P}\right|\right) \cdot r^{* 3} \\
& =\gamma^{*} \cdot M_{\Sigma}+\frac{E_{e m, e q}^{*}}{c^{2}} \frac{r_{e q}^{*}}{r^{*}}+\frac{1}{3}\left(\frac{E_{e m, e q}^{*}}{c^{2} r_{e q}^{* 3}}\right) \cdot r^{* 3}=\gamma^{*} \cdot M_{\Sigma}+\frac{E_{e m, e q}^{*}}{c^{2}}\left(\frac{r_{e q}^{*}}{r^{*}}+\frac{1}{3} \frac{r^{* 3}}{r_{e q}^{* 3}}\right) \\
& =>M=\gamma^{*} \cdot M_{\Sigma}+\frac{E_{e m, e q}^{*}}{c^{2}}\left(\frac{r_{e q}^{*}}{r^{*}}+\frac{1}{3} \frac{r^{* 3}}{r_{e q}^{* 3}}\right)
\end{aligned}
$$

The mass is a function $M=M\left(r^{*}, \frac{d r^{*}}{d t}, V_{\mathbf{C}}\right)$

For the equilibrium point we have the well known result

$$
M_{e q}=\gamma^{*} \cdot M_{\Sigma}+\frac{4}{3} \frac{E_{e m, e q}^{*}}{c^{2}}=\left(M_{\Sigma}+\frac{4}{3} \frac{E_{e m, e q}^{*}}{c^{2}}\right)_{i f \frac{d r^{*}}{d t}=0}
$$

Hence, as already many time said, for example in [3] \& [9], the a priori astonishing factor mass $\frac{4}{3}$ is due to the necessity of a confining term (Poincaré term) which add an energical contribution.

$$
M_{e q}=\left(\gamma^{*} \cdot M_{\Sigma}\right)_{\text {Material points }}+\left(\frac{E_{e m, e q}^{*}}{c^{2}}\right)_{\text {Electromagnetic field }}+\left(\frac{1}{3} \frac{E_{e m, e q}^{*}}{c^{2}}\right)_{\text {Poincaré confinment }}
$$

The internal equation of motion of the sphere in second order approximation

We clarify the following factor

$$
\begin{aligned}
\gamma^{*}=\gamma^{*}\left(\boldsymbol{V}_{\mathbf{C}}, \frac{d r^{*}}{d t}\right) & =\frac{1}{\sqrt{1-\frac{\gamma\left(\boldsymbol{V}_{\mathbf{C}}\right)^{2}}{c^{2}}\left(\frac{d r^{*}}{d t}\right)^{2}}}=\frac{1}{\sqrt{\frac{1-\frac{\left(\boldsymbol{V}_{\mathbf{C}}\right)^{2}}{c^{2}}-\frac{1}{c^{2}}\left(\frac{d r^{*}}{d t}\right)^{2}}{1-\frac{\left(\boldsymbol{V}_{\mathbf{C}}\right)^{2}}{c^{2}}}}=\sqrt{\frac{1-\frac{\left(\boldsymbol{V}_{\mathbf{C}}\right)^{2}}{c^{2}}}{1-\frac{\left(\boldsymbol{V}_{\mathbf{C}}\right)^{2}}{c^{2}}-\frac{1}{c^{2}}\left(\frac{d r^{*}}{d t}\right)^{2}}}} \\
& \approx\left(1-\frac{1}{2} \frac{\left(\boldsymbol{V}_{\mathbf{C}}\right)^{2}}{c^{2}}\right)\left(1+\frac{1}{2} \frac{\left(\boldsymbol{V}_{\mathbf{C}}\right)^{2}}{c^{2}}+\frac{1}{2} \frac{1}{c^{2}}\left(\frac{d r^{*}}{d t}\right)^{2}\right) \\
& =\left(1+\frac{1}{2} \frac{\boldsymbol{V}_{\mathbf{C}}{ }^{2}}{c^{2}}+\frac{1}{2} \frac{1}{c^{2}}\left(\frac{d r^{*}}{d t}\right)^{2}-\frac{1}{2} \frac{\boldsymbol{V}_{\mathbf{C}}{ }^{2}}{c^{2}}\left(1+\frac{1}{2} \frac{\boldsymbol{V}_{\mathbf{C}}{ }^{2}}{c^{2}}+\frac{1}{2} \frac{1}{c^{2}}\left(\frac{d r^{*}}{d t}\right)^{2}\right)\right) \\
& =1+\frac{1}{2} \frac{\boldsymbol{V}_{\mathbf{C}}{ }^{2}}{c^{2}}+\frac{1}{2} \frac{1}{c^{2}}\left(\frac{d r^{*}}{d t}\right)^{2}-\frac{1}{2} \frac{\boldsymbol{V}_{\mathbf{C}}{ }^{2}}{c^{2}}-\frac{1}{4} \frac{\boldsymbol{V}_{\mathbf{C}}{ }^{2}}{c^{2}} \frac{\boldsymbol{V}_{\mathbf{C}}{ }^{2}}{c^{2}}-\frac{1}{4} \frac{\boldsymbol{V}_{\mathbf{C}}{ }^{2}}{c^{2}} \frac{1}{c^{2}}\left(\frac{d r^{*}}{d t}\right)^{2}
\end{aligned}
$$




$$
\begin{gathered}
=>\gamma^{*}=1+\frac{1}{2} \frac{1}{c^{2}}\left(\frac{d r^{*}}{d t}\right)^{2}-\frac{1}{4} \frac{\left(\boldsymbol{V}_{\mathbf{C}}\right)^{4}}{c^{4}}-\frac{1}{4} \frac{\left(\boldsymbol{V}_{\mathbf{C}}\right)^{2}}{c^{2}} \frac{1}{c^{2}}\left(\frac{d r^{*}}{d t}\right)^{2} \\
=>\gamma^{*}=1+\frac{1}{2} \frac{1}{c^{2}}\left(\frac{d r^{*}}{d t}\right)^{2}+\theta\left(\frac{v^{4}}{c^{4}}\right)
\end{gathered}
$$

Then

$$
\begin{aligned}
& \frac{d}{d t}\left(\gamma\left(\boldsymbol{V}_{\mathbf{C}}\right) \cdot \gamma^{*} M_{\Sigma} \cdot \frac{d r^{*}}{d t}\right)=\frac{1}{\gamma\left(\boldsymbol{V}_{\mathbf{C}}\right)} E_{e m, e q}^{*}\left(\frac{r_{e q}^{*}}{r^{* 2}}-\frac{r^{* 2}}{r_{e q}^{* 3}}\right) \\
& <=>\frac{d}{d t}\left(\gamma\left(\boldsymbol{V}_{\mathbf{C}}\right)\left(1+\frac{1}{2} \frac{1}{c^{2}}\left(\frac{d r^{*}}{d t}\right)^{2}\right) M_{\Sigma} \frac{d r^{*}}{d t}\right) \approx \frac{1}{\gamma\left(\boldsymbol{V}_{\mathbf{C}}\right)} E_{e m, e q}^{*}\left(\frac{r_{e q}^{*}}{r^{* 2}}-\frac{r^{* 2}}{r_{e q}^{* 3}}\right) \\
& <=>\frac{d}{d t}\left(\gamma\left(\boldsymbol{V}_{\mathbf{C}}\right)\right) \gamma^{*} M_{\Sigma} \frac{d r^{*}}{d t}+\frac{d}{d t}\left(\left(1+\frac{1}{2} \frac{1}{c^{2}}\left(\frac{d r^{*}}{d t}\right)^{2}\right)\right) \gamma\left(\boldsymbol{V}_{\mathbf{C}}\right) M_{\Sigma} \frac{d r^{*}}{d t}+\frac{d}{d t}\left(\frac{d r^{*}}{d t}\right) \gamma\left(\boldsymbol{V}_{\mathbf{C}}\right) \gamma^{*} M_{\Sigma}=\frac{1}{\gamma\left(\boldsymbol{V}_{\mathbf{C}}\right)} E_{e m, e q}^{*}\left(\frac{r_{e q}^{*}}{r^{* 2}}-\frac{r^{* 2}}{r_{e q}^{* 3}}\right) \\
& <=>\frac{d}{d t}\left(\gamma\left(\boldsymbol{V}_{\mathbf{C}}\right)\right) \gamma^{*} M_{\Sigma} \frac{d r^{*}}{d t}+\frac{1}{c^{2}} \frac{d r^{*}}{d t} \frac{d^{2} r^{*}}{d t^{2}} \gamma\left(\boldsymbol{V}_{\mathbf{C}}\right) M_{\Sigma} \frac{d r^{*}}{d t}+\frac{d^{2} r^{*}}{d t^{2}} \gamma\left(\boldsymbol{V}_{\mathbf{C}}\right) \gamma^{*} M_{\Sigma}=\frac{1}{\gamma\left(\boldsymbol{V}_{\mathbf{C}}\right)} E_{e m, e q}^{*}\left(\frac{r_{e q}^{*}}{r^{* 2}}-\frac{r^{* 2}}{r_{e q}^{*}}\right) \\
& <=>\frac{d}{d t}\left(\gamma\left(\boldsymbol{V}_{\mathbf{C}}\right)\right) \frac{\gamma^{*}}{\gamma\left(\boldsymbol{V}_{\mathbf{C}}\right) \gamma^{*}} M_{\Sigma} \frac{d r^{*}}{d t}+\frac{1}{c^{2}} \frac{d r^{*}}{d t} \frac{d^{2} r^{*}}{d t^{2}} \frac{\gamma\left(\boldsymbol{V}_{\mathbf{C}}\right)}{\gamma\left(\boldsymbol{V}_{\mathbf{C}}\right) \gamma^{*}} M_{\Sigma} \frac{d r^{*}}{d t}+M_{\Sigma} \frac{d^{2} r^{*}}{d t^{2}}=\frac{1}{\gamma\left(\boldsymbol{V}_{\mathbf{C}}\right) \gamma\left(\boldsymbol{V}_{\mathbf{C}}\right) \gamma^{*}} E_{e m, e q}^{*}\left(\frac{r_{e q}^{*}}{r^{* 2}}-\frac{r^{* 2}}{r_{e q}^{* 3}}\right) \\
& <=>\left(\frac{1}{c^{2}} \frac{d r^{*}}{d t} \frac{1}{\gamma^{*}} M_{\Sigma} \frac{d r^{*}}{d t}+M_{\Sigma}\right) \frac{d^{2} r^{*}}{d t^{2}}=\frac{1}{\gamma\left(\boldsymbol{V}_{\mathbf{C}}\right) \gamma\left(\boldsymbol{V}_{\mathbf{C}}\right) \gamma^{*}} E_{e m, e q}^{*}\left(\frac{r_{e q}^{*}}{r^{* 2}}-\frac{r^{* 2}}{r_{e q}^{*}}\right)-\frac{d \ln \left(\gamma\left(\boldsymbol{V}_{\mathbf{C}}\right)\right)}{d t} M_{\Sigma} \frac{d r^{*}}{d t} \\
& <=>\left(1+\frac{1}{c^{2}}\left(\frac{d r^{*}}{d t}\right)^{2}\right) M_{\Sigma} \frac{d^{2} r^{*}}{d t^{2}} \approx \frac{1}{\gamma\left(\boldsymbol{V}_{\mathbf{C}}\right)^{2} \gamma^{*}} E_{e m, e q}^{*}\left(\frac{r_{e q}^{*}}{r^{* 2}}-\frac{r^{* 2}}{r_{e q}^{*}}\right)-M_{\Sigma} \frac{d \ln \left(\gamma\left(\boldsymbol{V}_{\mathbf{C}}\right)\right)}{d t} \frac{d r^{*}}{d t} \\
& \frac{d \ln \left(\gamma\left(\boldsymbol{V}_{\mathbf{C}}\right)\right)}{d t}=\frac{1}{\gamma\left(\boldsymbol{V}_{\mathbf{C}}\right)} \frac{d \gamma\left(\boldsymbol{V}_{\mathbf{C}}\right)}{d t}=\frac{1}{\gamma\left(\boldsymbol{V}_{\mathbf{C}}\right)} \frac{d\left(1-\left(\frac{\boldsymbol{V}_{\mathbf{C}}}{c}\right)^{2}\right)^{-1 / 2}}{d t}=\frac{1}{\gamma\left(\boldsymbol{V}_{\mathbf{C}}\right)} \frac{-1}{2}\left(1-\left(\frac{\boldsymbol{V}_{\mathbf{C}}}{c}\right)^{2}\right)^{-3 / 2} \frac{d\left(1-\left(\frac{\boldsymbol{V}_{\mathbf{C}}}{c}\right)^{2}\right)}{d t} \\
& =\frac{1}{\gamma\left(\boldsymbol{V}_{\mathbf{C}}\right)} \frac{-1}{2} \gamma\left(\boldsymbol{V}_{\mathbf{C}}\right)^{3}\left(-2\left(\frac{1}{c^{2}}\right) \boldsymbol{V}_{\mathbf{C}} \boldsymbol{a}_{\mathbf{C}}\right)=\frac{1}{c^{2}} \gamma\left(\boldsymbol{V}_{\mathbf{C}}\right)^{2} \boldsymbol{V}_{\mathbf{C}} \boldsymbol{a}_{\mathbf{C}} \\
& =>\left(1+\frac{1}{c^{2}}\left(\frac{d r^{*}}{d t}\right)^{2}\right) M_{\Sigma} \frac{d^{2} r^{*}}{d t^{2}} \approx \frac{1}{\gamma\left(\boldsymbol{V}_{\mathbf{C}}\right)^{2} \gamma^{*}} E_{e m, e q}^{*}\left(\frac{r_{e q}^{*}}{r^{* 2}}-\frac{r^{* 2}}{r_{e q}^{* 3}}\right)-\frac{M_{\Sigma}}{c^{2}} \gamma\left(\boldsymbol{V}_{\mathbf{C}}\right)^{2} \boldsymbol{V}_{\mathbf{C}} \boldsymbol{a}_{\mathbf{C}} \frac{d r^{*}}{d t} \\
& <=>M_{\Sigma} \frac{d^{2} r^{*}}{d t^{2}} \approx \frac{1}{\gamma\left(\boldsymbol{V}_{\mathbf{C}}\right)^{2} \gamma^{*}}\left(1-\frac{1}{c^{2}}\left(\frac{d r^{*}}{d t}\right)^{2}\right) E_{e m, e q}^{*}\left(\frac{r_{e q}^{*}}{r^{* 2}}-\frac{r^{* 2}}{r_{e q}^{*}}\right)-\frac{M_{\Sigma}}{c^{2}}\left(1-\frac{1}{c^{2}}\left(\frac{d r^{*}}{d t}\right)^{2}\right) \gamma\left(\boldsymbol{V}_{\mathbf{C}}\right)^{2} \boldsymbol{V}_{\mathbf{C}} \boldsymbol{a}_{\mathbf{C}} \frac{d r^{*}}{d t} \\
& M_{\Sigma} \frac{d^{2} r^{*}}{d t^{2}}=-\alpha \cdot \frac{d r^{*}}{d t}+\vartheta \cdot E_{e m, e q}^{*}\left(\frac{r_{e q}^{*}}{r^{* 2}}-\frac{r^{* 2}}{r_{e q}^{* 3}}\right) \\
& \text { - } \alpha=\alpha\left(\boldsymbol{V}_{\mathbf{C}}, \frac{d r^{*}}{d t}\right)=\frac{M_{\Sigma}}{c^{2}}\left(1-\frac{1}{c^{2}}\left(\frac{d r^{*}}{d t}\right)^{2}\right) \gamma\left(\boldsymbol{V}_{\mathbf{C}}\right)^{2} \boldsymbol{V}_{\mathbf{C}} \boldsymbol{a}_{\mathbf{C}} \\
& \text { - } \vartheta=\vartheta\left(\boldsymbol{V}_{\mathbf{C}}, \frac{d r^{*}}{d t}\right)=\frac{1}{\gamma\left(\boldsymbol{V}_{\mathbf{C}}\right)^{2} \gamma^{*}}\left(1-\frac{1}{c^{2}}\left(\frac{d r^{*}}{d t}\right)^{2}\right)
\end{aligned}
$$

With this effective Newtonian form one can interpret more intuitively the internal relativistic equation where we notice: 
- a viscous term $-\alpha \cdot \frac{d r^{*}}{d t}$ with a coefficient $\alpha$ proportional to $\boldsymbol{V}_{\mathrm{C}} \cdot \boldsymbol{a}_{\mathrm{C}}$, in accordance with the conservation of the energy (exchange between internal energy and the kinetic energy)

- a factor $\vartheta$ affecting the repulsive Coulombian force and the pressure force

We see that in general there is a coupling between the external dynamic and the internal dynamic. But this coupling is clearly due to the relativistic regime: outside this regime, the external dynamic does not affect the internal dynamic (since $K^{*}$ is a local Galilean frame, there are no inertial forces).

The case of a non-relativistic internal dynamic $\left(\frac{1}{c} \frac{d r^{*}}{d t} \approx 0\right)$ gives:

- $\quad \alpha=\alpha\left(\boldsymbol{V}_{\mathbf{C}}, \frac{d r^{*}}{d t}\right) \approx \frac{M_{\Sigma}}{c^{2}} \gamma\left(\boldsymbol{V}_{\mathbf{C}}\right)^{2} \boldsymbol{V}_{\mathbf{C}} \boldsymbol{a}_{\mathbf{C}}=\frac{M_{\Sigma}}{c^{2}} \frac{\boldsymbol{V}_{\mathrm{C}} \boldsymbol{a}_{\mathbf{C}}}{1-\frac{\boldsymbol{V}_{\mathbf{C}}{ }^{2}}{c^{2}}}$

- $\vartheta=\vartheta\left(\boldsymbol{V}_{\mathbf{C}}, \frac{d r^{*}}{d t}\right)=\frac{1}{\gamma\left(\boldsymbol{V}_{\mathrm{C}}\right)^{2}}=1-\frac{\boldsymbol{V}_{\mathbf{C}}{ }^{2}}{c^{2}}$

$\Rightarrow>\alpha \frac{d r^{*}}{d t}=\frac{M_{\Sigma}}{c^{2}} \frac{V_{\mathrm{C}} a_{\mathrm{C}}}{1-\frac{V_{\mathrm{C}}}{c^{2}}} \frac{d r^{*}}{d t}=K \cdot \frac{1}{c} \frac{d r^{*}}{d t} \approx 0$

$$
=>\left(M_{\Sigma} \frac{d^{2} r^{*}}{d t^{2}} \approx \frac{1}{\gamma\left(v_{\mathrm{c}}\right)^{2}} E_{e m, e q}^{*}\left(\frac{r_{e q}^{*}}{r^{* 2}}-\frac{r^{* 2}}{r_{e q}^{*}}\right)\right)_{\text {None-relativistic internal dynamic }}
$$

To simplify the dynamics, I will assume that the system is close to the equilibrium point. Then we can Taylor the function $f\left(r^{*}\right) \equiv \frac{r_{e q}^{*}}{r^{* 2}}-\frac{r^{* 2}}{r_{e q}^{*}}$ near this point.

$$
\begin{gathered}
f\left(r^{*}\right) \approx f\left(r_{e q}^{*}\right)+\frac{d f}{d r^{*}}\left(r_{e q}^{*}\right) \cdot\left(r^{*}-r_{e q}^{*}\right)=\left(\frac{r_{e q}^{*}}{r^{* 2}}-\frac{r^{* 2}}{r_{e q}^{* 3}}\right)\left(r_{e q}^{*}\right)+\left(-2 \frac{r_{e q}^{*}}{r^{* 3}}-2 \frac{r^{*}}{r_{e q}^{*} 3}\right)\left(r_{e q}^{*}\right) \cdot\left(r^{*}-r_{e q}^{*}\right) \\
=(0)-\frac{4}{r_{e q}^{* 2}} \cdot\left(r^{*}-r_{e q}^{*}\right) \\
=>M_{\Sigma} \frac{d^{2} r^{*}}{d t^{2}} \approx-\frac{4}{r_{e q}^{*}} \frac{1}{\gamma\left(v_{c}\right)^{2}} E_{e m, e q}^{*}\left(r^{*}-r_{e q}^{*}\right)
\end{gathered}
$$

If the speed of the center of mass varies sufficiently slowly (adiabatically), we have as desired the case of an effective oscillator around a center of mass velocity $\boldsymbol{V}_{\mathbf{C}}{ }^{2}$ :

$$
M_{\Sigma} \frac{d^{2} r^{*}}{d t^{2}} \approx-k_{V_{C^{*}}}\left(r^{*}-r_{e q}^{*}\right)
$$

With $k_{V_{\mathbf{C}}} \equiv \frac{4}{r_{e q}^{*}} \frac{1}{\gamma\left(V_{\mathbf{C}}\right)^{2}} E_{e m, e q}^{*}$

Taking into account $r_{e q}^{*}{ }^{3}=2 \frac{k}{\left|\Lambda_{P}\right| c^{4}} E_{e m, e q}^{*}$, the pulsation of the oscillator is then:

$$
\begin{aligned}
& \omega_{\boldsymbol{V}_{\mathbf{C}}}=\sqrt{\frac{k_{\boldsymbol{V}_{\mathbf{C}}}}{M_{\Sigma}}} \approx \frac{2}{r_{e q}^{*}} \sqrt{E_{e m, e q}^{*}} \frac{1}{\gamma\left(\boldsymbol{V}_{\mathbf{C}}\right)}=\frac{2 \sqrt{E_{e m, e q}^{*}}}{\left(2 \frac{k}{\left|\Lambda_{P}\right| c^{4}} E_{e m, e q}^{*}\right)^{1 / 3}} \frac{1}{\gamma\left(\boldsymbol{V}_{\mathrm{C}}\right)} \\
& =2^{2 / 3}\left(\frac{c^{4}\left|\Lambda_{P}\right|}{k}\right)^{1 / 3} E_{e m, e q}^{*} 1 / 6\left(1-\frac{1}{2} \frac{\boldsymbol{V}_{\mathbf{C}}{ }^{2}}{c^{2}}\right)
\end{aligned}
$$




$$
\omega_{V_{\mathbf{C}}} \approx \frac{\omega_{0}}{\gamma\left(V_{\mathbf{C}}\right)}=\omega_{0}+\Delta \omega_{V_{\mathbf{C}}}
$$

- $\omega_{0} \approx 2^{2 / 3}\left(\frac{c^{4}\left|\Lambda_{P}\right|}{k}\right)^{1 / 3} E_{e m, e q}^{*} 1 / 6$

- $\Delta \omega_{V_{\mathrm{C}}} \approx-\frac{1}{2} \frac{V_{\mathrm{C}}^{2}}{c^{2}} \omega_{0}$

Hence,

- if the internal system has Newtonian dynamics ;

- if the velocity of the center of mass is not negligible relative to the Einstein constant c ([5']);

- and if the speed of the center of mass varies sufficiently slowly with respect to the internal dynamics,

Then the internal oscillator sees its frequency $\omega_{V_{\mathbf{C}}}$ decreas to the value:

$$
-\Delta \omega_{V_{\mathbf{C}}} \approx \frac{1}{2} \frac{\boldsymbol{V}_{\mathbf{C}}{ }^{2}}{c^{2}} \omega_{0}
$$

A complex system whose center of mass moves at a sufficiently high speed affects the internal dynamics of the system.

The dynamic effect is actually a kinematic one

This, a priori dynamics effect, is actually rather a kinematic one, Einstein's law of time dilation:

$$
d t=\gamma\left(\boldsymbol{V}_{\mathbf{C}}\right) d t^{*}<=>\frac{1}{d t} \approx \frac{1}{\gamma\left(\boldsymbol{V}_{\mathbf{C}}\right)} \frac{1}{d t^{*}}
$$

We see in fact that the internal dynamic is frozen by the time dilation.

Indeed, we can re-express the internal dynamics in term of internal time $t^{*}$ by using the fact that:

$$
\begin{aligned}
& \frac{d^{2}}{d t^{2}}=\frac{d}{d t}\left(\frac{d}{d t}\right)=\frac{1}{\gamma\left(\boldsymbol{V}_{\mathbf{C}}\right)} \frac{d}{d t^{*}}\left(\frac{1}{\gamma\left(\boldsymbol{V}_{\mathbf{C}}\right)} \frac{d}{d t^{*}}\right) \\
& =\frac{1}{\gamma\left(\boldsymbol{V}_{\mathbf{C}}\right)}\left[\frac{d \gamma\left(\boldsymbol{V}_{\mathbf{C}}\right)^{-1}}{d t^{*}}\left(\frac{d}{d t^{*}}\right)+\frac{1}{\gamma\left(\boldsymbol{V}_{\mathbf{C}}\right)} \frac{d}{d t^{*}}\left(\frac{d}{d t^{*}}\right)\right] \\
& =\frac{1}{\gamma\left(\boldsymbol{V}_{\mathbf{C}}\right)}\left[\frac{d t}{d t^{*}} \frac{d \gamma\left(\boldsymbol{V}_{\mathbf{C}}\right)^{-1}}{d t}\left(\frac{d}{d t^{*}}\right)+\frac{1}{\gamma\left(\boldsymbol{V}_{\mathbf{C}}\right)} \frac{d}{d t^{*}}\left(\frac{d}{d t^{*}}\right)\right] \\
& =\frac{1}{\gamma\left(\boldsymbol{V}_{\mathbf{C}}\right)}\left[\gamma\left(\boldsymbol{V}_{\mathbf{C}}\right) \frac{d\left(1-\frac{\boldsymbol{V}_{\mathbf{C}}^{2}}{c^{2}}\right)^{1 / 2}}{d t}\left(\frac{d}{d t^{*}}\right)+\frac{1}{\gamma\left(\boldsymbol{V}_{\mathbf{C}}\right)} \frac{d}{d t^{*}}\left(\frac{d}{d t^{*}}\right)\right] \\
& =\frac{1}{\gamma\left(\boldsymbol{V}_{\mathbf{C}}\right)}\left[\gamma\left(\boldsymbol{V}_{\mathbf{C}}\right) \frac{1}{2}\left(-2 \frac{\boldsymbol{V}_{\mathbf{C}}}{c^{2}}\right) \boldsymbol{a}_{\mathbf{C}}\left(1-\frac{\boldsymbol{V}_{\mathbf{C}}{ }^{2}}{c^{2}}\right)^{-1 / 2}\left(\frac{d}{d t^{*}}\right)+\frac{1}{\gamma\left(\boldsymbol{V}_{\mathbf{C}}\right)} \frac{d}{d t^{*}}\left(\frac{d}{d t^{*}}\right)\right] \\
& =\frac{1}{\gamma\left(\boldsymbol{V}_{\mathbf{C}}\right)}\left[-\gamma\left(\boldsymbol{V}_{\mathbf{C}}\right)^{2} \frac{\boldsymbol{V}_{\mathbf{C}} \cdot \boldsymbol{a}_{\mathbf{C}}}{c^{2}}\left(\frac{d}{d t^{*}}\right)+\frac{1}{\gamma\left(\boldsymbol{V}_{\mathbf{C}}\right)} \frac{d}{d t^{*}}\left(\frac{d}{d t^{*}}\right)\right] \\
& =-\gamma\left(\boldsymbol{V}_{\mathbf{C}}\right) \frac{\boldsymbol{V}_{\mathbf{C} \cdot} \cdot \boldsymbol{a}_{\mathbf{C}}}{c^{2}}\left(\frac{d}{d t^{*}}\right)+\frac{1}{\gamma\left(\boldsymbol{V}_{\mathbf{C}}\right)^{2}} \frac{d}{d t^{*}}\left(\frac{d}{d t^{*}}\right) \\
& =>\frac{d^{2}}{d t^{2}}=-\gamma\left(\boldsymbol{V}_{\mathbf{C}}\right) \frac{\boldsymbol{V}_{\mathbf{C} \cdot} \boldsymbol{a}_{\mathbf{C}}}{c^{2}}\left(\frac{d}{d t^{*}}\right)+\frac{1}{\gamma\left(\boldsymbol{V}_{\mathbf{C}}\right)^{2}} \frac{d}{d t^{*}}\left(\frac{d}{d t^{*}}\right)
\end{aligned}
$$

Since we know that

$$
M_{\Sigma} \frac{d^{2} r^{*}}{d t^{2}} \approx \frac{1}{\gamma\left(\boldsymbol{V}_{\mathbf{C}}\right)^{2} \gamma^{*}}\left(1-\frac{1}{c^{2}}\left(\frac{d r^{*}}{d t}\right)^{2}\right) E_{e m, e q}^{*}\left(\frac{r_{e q}^{*}}{r^{* 2}}-\frac{r^{* 2}}{r_{e q}^{*}}\right)-\frac{M_{\Sigma}}{c^{2}}\left(1-\frac{1}{c^{2}}\left(\frac{d r^{*}}{d t}\right)^{2}\right) \gamma\left(\boldsymbol{V}_{\mathbf{C}}\right)^{2} \boldsymbol{V}_{\mathbf{C}} \boldsymbol{a}_{\mathbf{C}} \frac{d r^{*}}{d t}
$$


Then we have

$$
\begin{aligned}
& M_{\Sigma}\left(-\gamma\left(\boldsymbol{V}_{\mathbf{C}}\right) \frac{\boldsymbol{V}_{\mathbf{C}} \cdot \boldsymbol{a}_{\mathbf{C}}}{c^{2}}\left(\frac{d}{d t^{*}}\right)+\frac{1}{\gamma\left(\boldsymbol{V}_{\mathbf{C}}\right)^{2}} \frac{d}{d t^{*}}\left(\frac{d}{d t^{*}}\right)\right) r^{*} \\
& \approx \frac{1}{\gamma\left(\boldsymbol{V}_{\mathbf{C}}\right)^{2} \gamma^{*}}\left(1-\frac{1}{c^{2}}\left(\frac{d r^{*}}{d t}\right)^{2}\right) E_{e m, e q}^{*}\left(\frac{r_{e q}^{*}}{r^{* 2}}-\frac{r^{* 2}}{r_{e q}^{* 3}}\right)-\frac{M_{\Sigma}}{c^{2}}\left(1-\frac{1}{c^{2}}\left(\frac{d r^{*}}{d t}\right)^{2}\right) \gamma\left(\boldsymbol{V}_{\mathbf{C}}\right) \cdot \boldsymbol{V}_{\mathbf{C}} \boldsymbol{a}_{\mathbf{C}} \frac{d r^{*}}{d t^{*}} \\
& <=>M_{\Sigma}\left(-\gamma\left(\boldsymbol{V}_{\mathbf{C}}\right) \frac{\boldsymbol{V}_{\mathbf{C}} \cdot \boldsymbol{a}_{\mathbf{C}}}{c^{2}}\left(\frac{d r^{*}}{d t^{*}}\right)+\frac{1}{\gamma\left(\boldsymbol{V}_{\mathbf{C}}\right)^{2}} \frac{d}{d t^{*}}\left(\frac{d r^{*}}{d t^{*}}\right)\right) \\
& \approx \frac{1}{\gamma\left(\boldsymbol{V}_{\mathbf{C}}\right)^{2} \gamma^{*}}\left(1-\frac{1}{c^{2}}\left(\frac{d r^{*}}{d t}\right)^{2}\right) E_{e m, e q}^{*}\left(\frac{r_{e q}^{*}}{r^{* 2}}-\frac{r^{* 2}}{r_{e q}^{*}{ }^{3}}\right)-\frac{M_{\Sigma}}{c^{2}}\left(1-\frac{1}{c^{2}}\left(\frac{d r^{*}}{d t}\right)^{2}\right) \gamma\left(\boldsymbol{V}_{\mathbf{C}}\right) \cdot \boldsymbol{V}_{\mathbf{C}} \boldsymbol{a}_{\mathbf{C}} \frac{d r^{*}}{d t^{*}} \\
& <=>M_{\Sigma} \frac{1}{\gamma\left(\boldsymbol{V}_{\mathbf{C}}\right)^{2}} \frac{d}{d t^{*}}\left(\frac{d r^{*}}{d t^{*}}\right) \\
& \approx \frac{1}{\gamma\left(\boldsymbol{V}_{\mathbf{C}}\right)^{2} \gamma^{*}}\left(1-\frac{1}{c^{2}}\left(\frac{d r^{*}}{d t}\right)^{2}\right) E_{e m, e q}^{*}\left(\frac{r_{e q}^{*}}{r^{* 2}}-\frac{r^{* 2}}{r_{e q}^{* 3}}\right)-\frac{M_{\Sigma}}{c^{2}}\left[\left(1-\frac{1}{c^{2}}\left(\frac{d r^{*}}{d t}\right)^{2}\right)-1\right] \gamma\left(\boldsymbol{V}_{\mathbf{C}}\right) \boldsymbol{V}_{\mathbf{C}} \boldsymbol{a}_{\mathbf{C}} \frac{d r^{*}}{d t^{*}} \\
& <=>M_{\Sigma} \frac{d}{d t^{*}}\left(\frac{d r^{*}}{d t^{*}}\right) \approx \frac{1}{\gamma^{*}}\left(1-\frac{1}{c^{2}}\left(\frac{d r^{*}}{d t}\right)^{2}\right) E_{e m, e q}^{*}\left(\frac{r_{e q}^{*}}{r^{* 2}}-\frac{r^{* 2}}{r_{e q}^{*}{ }^{3}}\right)+\frac{M_{\Sigma}}{c^{2}} \frac{1}{c^{2}}\left(\frac{d r^{*}}{d t}\right)^{2} \gamma\left(\boldsymbol{V}_{\mathbf{C}}\right) \boldsymbol{V}_{\mathbf{C}} \boldsymbol{a}_{\mathbf{C}} \frac{d r^{*}}{d t^{*}} \\
& <=>M_{\Sigma} \frac{d}{d t^{*}}\left(\frac{d r^{*}}{d t^{*}}\right) \approx \frac{1}{\gamma^{*}}\left(1-\frac{1}{c^{2}}\left(\frac{d r^{*}}{d t}\right)^{2}\right) E_{e m, e q}^{*}\left(\frac{r_{e q}^{*}}{r^{* 2}}-\frac{r^{* 2}}{r_{e q}^{*}}\right)+\frac{M_{\Sigma}}{c^{2}} \frac{1}{c^{2}}\left(\frac{d r^{*}}{d t^{*}}\right)^{2} \frac{\boldsymbol{V}_{\mathbf{C}} \boldsymbol{a}_{\mathbf{C}}}{\gamma\left(\boldsymbol{V}_{\mathbf{C}}\right)} \frac{d r^{*}}{d t^{*}}
\end{aligned}
$$

$$
=>M_{\Sigma} \frac{d}{d t^{*}}\left(\frac{d r^{*}}{d t^{*}}\right)=\alpha \cdot \frac{d r^{*}}{d t^{*}}+\vartheta \cdot E_{e m, e q}^{*}\left(\frac{r_{e q}^{*}}{r^{* 2}}-\frac{r^{* 2}}{r_{e q}^{*}}\right)
$$

- $\alpha=\alpha\left(\boldsymbol{V}_{\mathbf{C}}, \frac{d r^{*}}{d t}\right)=\frac{M_{\Sigma}}{c^{2}} \frac{1}{c^{2}}\left(\frac{d r^{*}}{d t^{*}}\right)^{2} \frac{V_{\mathbf{C}} a_{\mathrm{C}}}{\gamma\left(\boldsymbol{V}_{\mathrm{C}}\right)}$

- $\vartheta=\vartheta\left(V_{\mathbf{C}}, \frac{d r^{*}}{d t}\right)=\frac{1}{r^{*}}\left(1-\frac{1}{c^{2}}\left(\frac{d r^{*}}{d t}\right)^{2}\right)$

\section{Digression on a (apparent ?) paradox}

The viscous term is a little embarrassing for me (Is there an error of sign or worse? I let the reader answer) because it shows a capture of the kinetic energy of the center of mass by the internal oscillator when the center of mass is accelerated. This is the complete opposite, at first sight, of the expected behaviour as seen above from the reference frame $\mathrm{K}$ with time $\mathrm{t}$. This seems to contradict the energy conservation law.

But in the case where the whole system is isolated, we know that the center of mass and the total energy has to be constant, so the viscous term disappears and there is no contradiction.

However, let's take this result seriously. One can imagine an external field acting on the whole system in a such way that the field is considered as totally homogenous from the point of view of the internal particles but inhomogeneous from the one of the center of mass. Then the external field accelerates the electron without "touching" the internal part. This results in an acceleration of the center of mass. As we have shown above, this has the effect of injecting energy from the center of mass to the internal energy, and thus increasing the mass. The consequence of this is that any acceleration of our electron increases its mass: "acceleration generates matter". This remind me of some claims readen in more sophisticated and general physics about creation of matter during "early" Big Bang phase of (more or less) speculative inflation. Our model, although less general (and physically false!), shows how one can understand pedagogically (=simple and explicit) this kind of behaviour in Special Relativity without field theory (if there are not errors of signs...).

To finish this digression, we can interpret the equation in the follows. From the point of view of internal dynamics, an acceleration of the center of mass increases the speed of the oscillator. But from the point of view of the observer $\mathrm{K}$, it is 
the opposite. As the difference between the 2 point of view comes from the dilation of time, we can say that when we increase the speed of the center of mass, in the point of view of $K$, the dilation of time increases sufficiently strongly to contrebalance the internal acceleration (seen from $\mathrm{K}^{*}$ ) and actually provide the result of an decelerated oscillator seen in $\mathrm{K}$. If there is no sign error (or worse), I found this result quite interesting as an apparent paradox in Special Relaticity.

Returning to our Like above, Once again, to simplify the dynamics, I will assume that

0 if the internal system has Newtonian dynamics ;

- the system is close to the equilibrium point.

$\circ$ the speed of the center of mass varies slowly (adiabatically):

$$
=>M_{\Sigma} \frac{d}{d t^{*}}\left(\frac{d r^{*}}{d t^{*}}\right) \approx-k_{\mathbf{0}} \cdot\left(r^{*}-r_{e q}^{*}\right)
$$

With $k_{\mathbf{0}} \equiv \frac{4}{r_{e q}^{*}{ }^{2}} E_{e m, e q}^{*}$

Then

$$
M_{\Sigma} \frac{d^{2} r^{*}}{d t^{2}} \approx-\frac{4}{r_{e q}^{* 2}} \frac{1}{\gamma\left(V_{\mathbf{C}}\right)^{2}} \frac{E_{e m, e q}^{*}}{M_{\Sigma}}\left(r^{*}-r_{e q}^{*}\right)<=>M_{\Sigma} \frac{d^{2} r^{*}}{d t^{*^{2}}} \approx-\frac{4}{r_{e q}^{* 2}} \frac{E_{e m, e q}^{*}}{M_{\Sigma}}\left(r^{*}-r_{e q}^{*}\right)
$$

In this form, we see that the dynamics is fully expressed in terms of variables in $\mathrm{K}^{*}$ without the dilation of time factor.

In this expression, the Hooke force is now characterized by $k_{\mathbf{0}}=\frac{4}{r_{e q}^{*}} \frac{E_{e m, e q}^{*}}{M_{\Sigma}}=\gamma\left(\boldsymbol{V}_{\mathbf{C}}\right)^{2} k_{\boldsymbol{V}_{\mathbf{C}}}$

We recover the expression above $\omega_{\mathbf{0}}=\sqrt{\frac{k_{0}}{M_{\Sigma}}}=\sqrt{\gamma\left(\boldsymbol{V}_{\mathbf{C}}\right)^{2} \frac{k_{V_{\mathbf{C}}}}{M_{\Sigma}}}=\gamma\left(\boldsymbol{V}_{\mathbf{C}}\right) \sqrt{\frac{k_{V_{\mathbf{C}}}}{M_{\Sigma}}}=\gamma\left(\boldsymbol{V}_{\mathbf{C}}\right) \omega_{\boldsymbol{V}_{\mathbf{C}}}$

Thus, the Newtonian oscillator is seen frozen à la Einstein by our new weird choice of variables that express the dynamics relative to the time $t$. This indicates naturally that the observer is in the reference frame $\mathrm{K}$.

Anticipating a quantum treatment that might be based on the new choice of variable, one can affirm that the quantum characteristics of the inner part (seen from $\mathrm{K}$ ) would be:

- The quantum of energy $\Delta E=\hbar \omega_{V_{\mathbf{C}}}=\hbar \frac{\omega_{0}}{\gamma\left(\boldsymbol{V}_{\mathrm{C}}\right)} \approx \hbar \omega_{\mathbf{0}}+\hbar \Delta \omega_{\boldsymbol{V}_{\mathbf{C}}}$;

- The zero point energy $E_{0}=\frac{1}{2} \hbar \omega_{\boldsymbol{V}_{\mathbf{C}}}=\frac{1}{2} \hbar \frac{\omega_{0}}{\gamma\left(\boldsymbol{V}_{\mathbf{C}}\right)} \approx \frac{1}{2} \hbar \omega_{\mathbf{0}}+\frac{1}{2} \hbar \Delta \omega_{\boldsymbol{V}_{\mathbf{C}}}$.

The "external relativistic dynamic" affects, by the relativity of time, the quantum of energy and the lowest energy ("zero point energy") by "renormalizing" them in a kinematic way.

Then if we measure this quantum and this zero point energy among a macroscopic number of such free complex "electrons" we naturally obtain the average values (thanks to the Equirepartition theorem):

- $\langle\Delta E\rangle=\hbar\left\langle\omega_{V_{\mathbf{C}}}\right\rangle=\hbar \omega_{0}\left\langle\frac{1}{\gamma\left(V_{\mathbf{C}}\right)}\right\rangle \approx \hbar \omega_{0}\left(1-\frac{1}{M c^{2}}\left\langle\frac{1}{2} M{V_{\mathrm{C}}}^{2}\right\rangle\right)=\hbar \omega_{\mathbf{0}}\left(1-\frac{3}{2} \frac{k_{B} T}{M c^{2}}\right)$;

- $\left\langle E_{0}\right\rangle=\frac{1}{2} \hbar\left\langle\omega_{V_{\mathrm{C}}}\right\rangle=\frac{1}{2} \hbar \omega_{0}\left\langle\frac{1}{\gamma\left(V_{\mathrm{C}}\right)}\right\rangle=\frac{1}{2} \hbar \omega_{0}\left(1-\frac{3}{2} \frac{k_{B} T}{M c^{2}}\right)$

And, if all the electrons are each in harmonic-Hooke interaction with a center of force, then the quantum characteristics measured are:

- $\langle\Delta E\rangle=\hbar\left\langle\omega_{V_{\mathrm{C}}}\right\rangle \approx \hbar \omega_{\mathbf{0}}\left(1-3 \frac{k_{B} T}{M c^{2}}\right)$;

- $\left\langle E_{0}\right\rangle \approx \frac{1}{2} \hbar \omega_{0}\left(1-3 \frac{k_{B} T}{M c^{2}}\right)$ 
Hence, from the point of view of an observer looking at a collection of moving "electron", the dilation of time, expressed by the thermal energy effect, decrease (a little) theses quantum characteristics of that particular electron. Because of the universality of the cause (Einstein dilation of time), this effect must also be true for other kind of material Newtonian oscillators (atoms, molecules...). It will be interesting to see the effect of the dilation of time for the quantum version of "particles" constituted, this time, only by fields (electromagnetism, gravitation fields...) which can be considered (at least for the first when it is free and the second for a weak field regime) as an infinity of oscillators, but this time the last system will be relativistic.

A question: For a wave packet travelling at the constant speed c, can we say that all the zero-point energy would be infinitely dilated and therefore "renormalized" (if internal energy has a meaning for a such system, a priori not but an explicit passage to the limit would be interesting to see) ?

\section{The mass}

In this situation the mass is now

$$
M=\gamma^{*} \cdot M_{\Sigma}+\frac{E_{e m, e q}^{*}}{c^{2}}\left(\frac{r_{e q}^{*}}{r^{*}}+\frac{1}{3} \frac{r^{* 3}}{r_{e q}^{* 3}}\right) \approx\left(1+\frac{1}{2} \frac{1}{c^{2}}\left(\frac{d r^{*}}{d t}\right)^{2}\right) \cdot M_{\Sigma}+\frac{E_{e m, e q}^{*}}{c^{2}} g\left(r^{*}\right)
$$

With the function $g\left(r^{*}\right) \equiv \frac{r_{e q}^{*}}{r^{*}}+\frac{1}{3} \frac{r^{* 3}}{r_{e q}^{*}}$

Taking account that for the equilibrium point $\left(\frac{d g}{d r^{*}}\right)_{r_{e q}^{*}}=0$, since the mass is an internal energy near the equilibrium point, we have

$$
\begin{gathered}
g\left(r^{*}\right)=g\left(r_{e q}^{*}\right)+\frac{1}{2}\left(\frac{d^{2} g}{d r^{* 2}}\right)_{r_{e q}^{*}}\left(r^{*}-r_{e q}^{*}\right)^{2}=\frac{4}{3}+\frac{1}{2}\left(\frac{d}{d r^{*}}\left[\frac{d}{d r^{*}}\left(\frac{r_{e q}^{*}}{r^{*}}+\frac{1}{3} \frac{r^{* 3}}{r_{e q}^{* 3}}\right)\right]\right)_{r_{e q}^{*}}\left(r^{*}-r_{e q}^{*}\right)^{2} \\
=\frac{4}{3}+\frac{1}{2}\left(\frac{d}{d r^{*}}\left(-\frac{r_{e q}^{*}}{r^{* 2}}+\frac{r^{* 2}}{r_{e q}^{* 3}}\right)\right)_{r_{e q}^{*}}\left(r^{*}-r_{e q}^{*}\right)^{2}=\frac{4}{3}+\frac{1}{2}\left(2 \frac{r_{e q}^{*}}{r^{* 3}}+2 \frac{r^{*}}{r_{e q}^{* 3}}\right)_{r_{e q}^{*}}\left(r^{*}-r_{e q}^{*}\right)^{2} \\
=\frac{4}{3}+\frac{1}{2}\left(2 \frac{1}{r_{e q}^{* 2}}+2 \frac{1}{r_{e q}^{* 2}}\right)\left(r^{*}-r_{e q}^{*}\right)^{2}=\frac{4}{3}+\frac{2}{r_{e q}^{* 2}}\left(r^{*}-r_{e q}^{*}\right)^{2} \\
=>M \approx\left(1+\frac{1}{2} \frac{1}{c^{2}}\left(\frac{d r^{*}}{d t}\right)^{2}\right) \cdot M_{\Sigma}+\frac{E_{e m, e q}^{*}}{c^{2}}\left[\frac{4}{3}+\frac{2}{r_{e q}^{* 2}}\left(r^{*}-r_{e q}^{*}\right)^{2}\right] \\
=\left(1+\frac{1}{2} \frac{1}{c^{2}}\left(\frac{d r^{*}}{d t}\right)^{2}\right) \cdot M_{\Sigma}+\frac{E_{e m, e q}^{*}}{c^{2}}\left[\frac{4}{3}+\frac{2}{r_{e q}^{* 2}}\left(r^{*}-r_{e q}^{*}\right)^{2}\right]
\end{gathered}
$$

\begin{tabular}{|c} 
With $=>M \approx M_{e q}+\frac{1}{2} \frac{1}{c^{2}}\left(\frac{d r^{*}}{d t}\right)^{2} \cdot M_{\Sigma}+\frac{E_{e m, e q}^{*}}{c^{2}} 2 \frac{\left(r^{*}-r_{e q}^{*}\right)^{2}}{r_{e q}^{*}}$ \\
$M_{e q}=M_{\Sigma}+\frac{4}{3} \frac{E_{e m, e q}^{*}}{c^{2}}$ \\
\hline
\end{tabular}


The mass is a function $M=M\left(r^{*}, \frac{d r^{*}}{d t}, V_{\mathbf{C}}\right)$.

The dynamic of the center of mass

and

$$
\frac{d}{d t}\left(\gamma\left(\boldsymbol{V}_{\mathbf{C}}\right) M \boldsymbol{V}_{\mathbf{C}}\right)=M \frac{d}{d t}\left(\gamma\left(\boldsymbol{V}_{\mathbf{C}}\right) \boldsymbol{V}_{\mathbf{C}}\right)+\left(\gamma\left(\boldsymbol{V}_{\mathbf{C}}\right) \boldsymbol{V}_{\mathbf{C}}\right) \frac{d M}{d t}
$$

$$
\begin{aligned}
& \frac{d M}{d t}=\frac{d}{d t}\left(M_{e q}+\frac{1}{2} \frac{1}{c^{2}}\left(\frac{d r^{*}}{d t}\right)^{2} \cdot M_{\Sigma}+\frac{E_{e m, e q}^{*}}{c^{2}} 2 \frac{\left(r^{*}-r_{e q}^{*}\right)^{2}}{r_{e q}^{* 2}}\right) \\
& =\frac{1}{c^{2}} \frac{d r^{*}}{d t} \frac{d^{2} r^{*}}{d t^{2}} \cdot M_{\Sigma}+\frac{E_{e m, e q}^{*}}{c^{2}} 4 \frac{\left(r^{*}-r_{e q}^{*}\right)}{r_{e q}^{*}} \frac{d r^{*}}{d t} \\
& \text { But } \frac{d^{2} r^{*}}{d t^{2}} \approx-\left(1-\frac{V_{\mathrm{C}}{ }^{2}}{c^{2}}\right) 4 \frac{E_{e m, e q}^{*}}{M_{\Sigma}} \frac{\left(r^{*}-r_{e q}^{*}\right)}{r_{e q}^{*}} \\
& =>\frac{d M}{d t}=\frac{d r^{*}}{d t}\left(-\frac{1}{c^{2}}\left(1-\frac{\boldsymbol{V}_{\mathbf{C}}{ }^{2}}{c^{2}}\right) 4 E_{e m, e q}^{*} \frac{\left(r^{*}-r_{e q}^{*}\right)}{r_{e q}^{*}}\right)+\frac{E_{e m, e q}^{*}}{c^{2}} 4 \frac{\left(r^{*}-r_{e q}^{*}\right)}{r_{e q}^{*}} \frac{d r^{*}}{d t} \\
& =\frac{1}{c^{2}} \frac{d r^{*}}{d t} \frac{\boldsymbol{V}_{\mathbf{C}}{ }^{2}}{c^{2}} 4 E_{e m, e q}^{*} \frac{\left(r^{*}-r_{e q}^{*}\right)}{r_{e q}^{* 2}} \\
& \text { But } \frac{d}{d t}\left(\gamma\left(\boldsymbol{V}_{\mathbf{C}}\right) M \boldsymbol{V}_{\mathbf{C}}\right)=\frac{\partial}{\partial \boldsymbol{R}_{\mathbf{C}}} L^{\prime}\left(r^{*}, \frac{d r^{*}}{d t}, \boldsymbol{R}_{\mathbf{C}}, \boldsymbol{V}_{\mathbf{C}}, t\right) \\
& =>M \frac{d}{d t}\left(\gamma\left(\boldsymbol{V}_{\mathbf{C}}\right) \boldsymbol{V}_{\mathbf{C}}\right)+\left(\gamma\left(\boldsymbol{V}_{\mathbf{C}}\right) \boldsymbol{V}_{\mathbf{C}}\right) \frac{d M}{d t}=\frac{\partial}{\partial \boldsymbol{R}_{\mathbf{C}}} L^{\prime}\left(r^{*}, \frac{d r^{*}}{d t}, \boldsymbol{R}_{\mathbf{C}}, \boldsymbol{V}_{\mathbf{C}}, t\right)
\end{aligned}
$$

Then

$$
M \frac{d}{d t}\left(\gamma\left(\boldsymbol{V}_{\mathbf{C}}\right) \boldsymbol{V}_{\mathbf{C}}\right)=\frac{\partial}{\partial \boldsymbol{R}_{\mathbf{C}}} L^{\prime}\left(r^{*}, \frac{d r^{*}}{d t}, \boldsymbol{R}_{\mathbf{C}}, \boldsymbol{V}_{\mathbf{C}}, t\right)-\alpha \cdot \boldsymbol{V}_{\mathbf{C}}
$$

with

- $\quad \alpha=\gamma\left(\boldsymbol{V}_{\mathbf{C}}\right) \frac{d M}{d t}=\frac{d M}{d t^{*}}$

- $\frac{d M}{d t}=\left(\frac{V_{\mathrm{C}}{ }^{2}}{c^{2}}\right)\left(\frac{4}{r_{e q}^{*}} \frac{E_{e m, e q}^{*}}{c^{2}}\left(r^{*}-r_{e q}^{*}\right) \frac{d r^{*}}{d t}\right)$

The internal dynamics influence the dynamics of the center of mass. This coupling is not due to an eventual relativistic behaviour of the internal dynamics but especially to the relativistic behaviour of the center of mass itself. Indeed, we see that in the Newtonian limit $\left(\frac{V_{\mathrm{C}}{ }^{2}}{c^{2}}=0\right)$, there is no longer a viscous term where the coupling appears. This coupling is of course due to an exchange between the internal energy $M$ and that of the center of mass. This variation of the internal energy modifies the inertia and then acts on the speed for a given momentum.

We can for example write:

$$
\frac{d M}{d t}=\left(\frac{\boldsymbol{V}_{\mathbf{C}}{ }^{2}}{c^{2}}\right)\left(\frac{4}{r_{e q}^{* 2}} \frac{E_{e m, e q}^{*}}{c^{2}}\left(r^{*}-r_{e q}^{*}\right) \frac{d r^{*}}{d t}\right)=\left(\frac{\boldsymbol{V}_{\mathbf{C}}{ }^{2}}{c^{2}}\right)\left(\gamma\left(\boldsymbol{V}_{\mathbf{C}}\right)^{2} k_{\boldsymbol{V}_{\mathbf{C}}}\left(r^{*}-r_{e q}^{*}\right) \frac{d r^{*}}{d t}\right)
$$




$$
=\left(\frac{\boldsymbol{V}_{\mathbf{C}}^{2}}{c^{2}}\right) \gamma\left(\boldsymbol{V}_{\mathbf{C}}\right)^{2} \cdot F \frac{d r^{*}}{d t} \propto F \frac{d r^{*}}{d t}=P
$$

With

$\circ \quad k_{V_{\mathrm{C}}} \equiv \frac{4}{r_{e q}^{*}} \frac{1}{\gamma\left(V_{\mathrm{C}}\right)^{2}} E_{e m, e q}^{*}$

○ The force $F \equiv M_{\Sigma} \frac{d^{2} r^{*}}{d t^{2}} \approx-\frac{4}{r_{e q}^{*}} \frac{1}{\gamma\left(v_{\mathrm{c}}\right)^{2}} \frac{E_{e m, e q}^{*}}{M_{\Sigma}}\left(r^{*}-r_{e q}^{*}\right)=-\frac{4}{r_{e q}^{*}{ }^{2}} \frac{1}{\gamma\left(v_{\mathrm{c}}\right)^{2}} \frac{E_{e m, e q}^{*}}{M_{\Sigma}}\left(r^{*}-r_{e q}^{*}\right)$

The power $\mathrm{P}=F \frac{d r^{*}}{d t}$

When the electron absorb energy $(P>0)$, then the viscous force opposes to the movement of the center of mass $(\alpha>0)$. 


\subsection{Hamiltonian analysis: Hamilton-Jacobi equation(an attempt) for a material system free}

As for [1] and [2], we start from the norm equation:

$$
\left(\frac{E^{*}}{c}\right)^{2}=\left(\frac{E}{c}\right)^{2}-P^{2}
$$

We have to express the different quantities in term of the action. For that, I search the expression of the action as the function of coordinates : that is to say the action resulting from the injection of the equation of motion in its variation. I need the 2 expressions below in term of coordinate:

$\circ$ By Mixing internal and external degree of freedom $\left(\left\{\boldsymbol{r}_{\boldsymbol{a}}^{*}\right\}, \boldsymbol{R}_{\boldsymbol{c}}, t\right)$

$\circ$ And only using internal degree of freedom $\left(\left\{\boldsymbol{r}_{\boldsymbol{a}}^{*}\right\}, t^{*}\right)$

- $S\left(\left\{\boldsymbol{r}_{\boldsymbol{a}}^{*}\right\}, \boldsymbol{R}_{\boldsymbol{c}}, t\right) \equiv\left\{S\left[\left\{\boldsymbol{r}_{\boldsymbol{a}}^{*}\left(t^{*}\right)\right\}, \boldsymbol{R}_{\boldsymbol{c}}, t\right]\right\}_{\text {real trajectory }}=\int_{t_{1}}^{t,\left\{\boldsymbol{r}_{\boldsymbol{a}}^{*}\right\}, \boldsymbol{R}_{\boldsymbol{c}}} \frac{L^{*}}{\gamma} \cdot d t=\int_{t_{1}}^{t,\left\{\boldsymbol{r}_{\boldsymbol{a}}^{*}\right\}, \boldsymbol{R}_{\boldsymbol{c}}} L^{\prime} \cdot d t$ $=>\delta S\left(\left\{\boldsymbol{r}_{\boldsymbol{a}}^{*}\right\}, \boldsymbol{R}_{\boldsymbol{c}}, t\right)$

$$
\begin{aligned}
& =\int_{t_{1}}^{t,\left\{\boldsymbol{r}_{\boldsymbol{a}}^{*}\right\}, \boldsymbol{R}_{\boldsymbol{c}}}\left(\sum_{a} \frac{\partial L^{\prime}}{\partial \boldsymbol{r}_{\boldsymbol{a}}^{*}} \delta \boldsymbol{r}_{\boldsymbol{a}}^{*}+\sum_{a} \frac{\partial L^{\prime}}{\partial \frac{d \boldsymbol{r}_{\boldsymbol{a}}^{*}}{d t}} \delta \frac{d \boldsymbol{r}_{\boldsymbol{a}}^{*}}{d t}+\frac{\partial L^{\prime}}{\partial \boldsymbol{R}_{\mathbf{C}}} \delta \boldsymbol{R}_{\mathbf{C}}+\frac{\partial L^{\prime}}{\partial \boldsymbol{V}_{\mathbf{C}}} \delta \boldsymbol{V}_{\mathrm{C}}+\frac{\partial L^{\prime}}{\partial t} \delta t\right) d t \\
& =\int_{t_{1}}^{t,\left\{\boldsymbol{r}_{\boldsymbol{a}}^{*}\right\}, \boldsymbol{R}_{\boldsymbol{c}}}\left(\sum_{a} \frac{\partial L^{\prime}}{\partial \boldsymbol{r}_{\boldsymbol{a}}^{*}} \delta \boldsymbol{r}_{\boldsymbol{a}}^{*}+\frac{d}{d t}\left(\sum_{a} \frac{\partial L^{\prime}}{\partial \frac{d \boldsymbol{r}_{\boldsymbol{a}}^{*}}{d t}} \delta \boldsymbol{r}_{\boldsymbol{a}}^{*}-\delta \boldsymbol{r}_{\boldsymbol{a}}^{*} \frac{d}{d t} \sum_{a} \frac{\partial L^{\prime}}{\partial \frac{d \boldsymbol{r}_{\boldsymbol{a}}^{*}}{d t}}\right)+\frac{\partial L^{\prime}}{\partial \boldsymbol{R}_{\mathrm{C}}} \delta \boldsymbol{R}_{\mathrm{C}}\right. \\
& \left.+\left(\frac{d}{d t}\left(\frac{\partial L^{\prime}}{\partial v_{\mathbf{C}}} \delta \boldsymbol{R}_{\mathbf{C}}\right)-\delta \boldsymbol{R}_{\mathbf{C}}\left(\frac{d}{d t} \frac{\partial L^{\prime}}{\partial v_{\mathbf{C}}}\right)\right)+\frac{\partial L^{\prime}}{\partial t} \delta t\right) d t \\
& =\int_{t_{1}}^{t,\left\{\boldsymbol{r}_{\boldsymbol{a}}^{*}\right\}, \boldsymbol{R}_{\boldsymbol{c}}}\left\{\sum_{a}\left(\frac{\partial L^{\prime}}{\partial \boldsymbol{r}_{\boldsymbol{a}}^{*}}-\frac{d}{d t} \frac{\partial L^{\prime}}{\partial \frac{d \boldsymbol{r}_{\boldsymbol{a}}^{*}}{d t}}\right) \delta \boldsymbol{r}_{\boldsymbol{a}}^{*}+\left(\frac{\partial L^{\prime}}{\partial \boldsymbol{R}_{\mathrm{C}}}-\frac{d}{d t} \frac{\partial L^{\prime}}{\partial v_{\mathrm{C}}}\right) \delta \boldsymbol{R}_{\mathrm{C}}+\frac{\partial L^{\prime}}{\partial t} \delta t\right. \\
& \left.+\frac{d}{d t}\left(\sum_{a} \frac{\partial L^{\prime}}{\partial \frac{d \boldsymbol{r}_{a}^{*}}{d t}} \delta \boldsymbol{r}_{\boldsymbol{a}}^{*}+\frac{\partial L^{\prime}}{\partial \boldsymbol{v}_{\mathbf{C}}} \delta \boldsymbol{R}_{\mathbf{C}}\right)\right\} d t \\
& =>\delta S\left(\left\{\boldsymbol{r}_{\boldsymbol{a}}^{*}\right\}, \boldsymbol{R}_{\boldsymbol{c}}, t\right)=\int_{t_{1}}^{t,\left\{\delta \boldsymbol{r}_{\boldsymbol{a}}^{*}\right\}, \delta \boldsymbol{R}_{\mathrm{C}}} d\left(\sum_{a} \frac{\partial L^{\prime}}{\partial \frac{d \boldsymbol{r}_{\boldsymbol{a}}^{*}}{d t}} \delta \boldsymbol{r}_{\boldsymbol{a}}^{*}+\frac{\partial L^{\prime}}{\partial \boldsymbol{V}_{\mathrm{C}}} \delta \boldsymbol{R}_{\mathrm{C}}\right)
\end{aligned}
$$

Since

- $\left(\frac{\partial L^{\prime}}{\partial r_{a}^{*}}-\frac{d}{d t} \frac{\partial L}{\partial \frac{d r_{a}^{*}}{d t}}=0\right)_{\text {for a real trajectory }}$

- $\left(\frac{\partial L}{\partial \boldsymbol{R}_{\mathrm{C}}}-\frac{d}{d t} \frac{\partial L^{\prime}}{\partial V_{\mathrm{C}}}=0\right)_{\text {for a real trajectory }}$

Then we have the following result 


$$
\begin{gathered}
=>d S\left(\left\{\boldsymbol{r}_{\boldsymbol{a}}^{*}\right\}, \boldsymbol{R}_{\boldsymbol{c}}, t\right)=\sum_{a}\left(\frac{\partial S}{\partial \boldsymbol{r}_{\boldsymbol{a}}^{*}} d \boldsymbol{r}_{\boldsymbol{a}}^{*}\right)+\frac{\partial S}{\partial \boldsymbol{R}_{\mathrm{C}}} d \boldsymbol{R}_{\mathbf{C}}+\frac{\partial S}{\partial t} d t \\
=\sum_{a} \frac{\partial L^{\prime}}{\partial \frac{d \boldsymbol{r}_{\boldsymbol{a}}^{*}}{d t}} d \boldsymbol{r}_{\boldsymbol{a}}^{*}+\frac{\partial L^{\prime}}{\partial \boldsymbol{v}_{\mathrm{C}}} d \boldsymbol{R}_{\mathbf{C}}+\frac{\partial S}{\partial t} d t \\
=\sum_{a} \boldsymbol{P}_{\boldsymbol{a}}^{*} d \boldsymbol{r}_{\boldsymbol{a}}^{*}+\boldsymbol{P} d \boldsymbol{R}_{\mathbf{C}}+\frac{\partial S}{\partial t} d t
\end{gathered}
$$

Then

- $\boldsymbol{P}_{\boldsymbol{a}}^{*} \equiv \frac{\partial L^{\prime}}{\partial \frac{d r_{a}^{*}}{d t}}=\frac{\partial S}{\partial \boldsymbol{r}_{\boldsymbol{a}}^{*}}$

- $\quad \boldsymbol{P} \equiv \frac{\partial L^{\prime}}{\partial v_{\mathrm{C}}}=\frac{\partial S}{\partial R_{\mathrm{C}}}$

- $L^{\prime} \equiv \frac{d S}{d t}\left(\left\{\boldsymbol{r}_{\boldsymbol{a}}^{*}\right\}, \boldsymbol{R}_{\boldsymbol{c}}, t\right)$

- $H\left(\left\{\boldsymbol{r}_{\boldsymbol{a}}^{*}\right\},\left\{\boldsymbol{P}_{\boldsymbol{a}}^{*}\right\}, \boldsymbol{R}_{\boldsymbol{c}}, \boldsymbol{V}_{\boldsymbol{c}}\right) \equiv \sum_{a} \boldsymbol{P}_{\boldsymbol{a}}^{*} \frac{d \boldsymbol{r}_{\boldsymbol{a}}^{*}}{d t}+\boldsymbol{P} \boldsymbol{V}_{\boldsymbol{c}}-L^{\prime}=\frac{\partial S}{\partial t}$

- $S\left(\left\{\boldsymbol{r}_{\boldsymbol{a}}^{*}\right\}, \boldsymbol{R}_{\boldsymbol{c}}, t\right) \equiv\left\{S\left[\left\{\boldsymbol{r}_{\boldsymbol{a}}^{*}\left(t^{*}\right)\right\}, \boldsymbol{R}_{\boldsymbol{c}}(t)\right]\right\}_{\text {real trajectory }}$

- $S\left(\left\{\boldsymbol{r}_{\boldsymbol{a}}^{*}\right\}, t^{*}\right)=\left\{S\left[\left\{\boldsymbol{r}_{\boldsymbol{a}}^{*}\left(t^{*}\right), t^{*}(t)\right\}\right]\right\}_{\text {real trajectory }}=\int_{t_{1}^{*}}^{t^{*},\left\{\boldsymbol{r}_{\boldsymbol{a}}^{*}\right\}} L^{*} d t^{*}$

$$
\begin{aligned}
& \delta S\left(\left\{\boldsymbol{r}_{\boldsymbol{a}}^{*}\right\}, t^{*}\right)=\left\{\delta S\left[\left\{\boldsymbol{r}_{\boldsymbol{a}}^{*}\left(t^{*}\right)\right\}, t^{*}(t)\right]\right\}_{\text {real trajectory }}=\int_{t_{1}^{*}}^{t^{*},\left\{\boldsymbol{r}_{\boldsymbol{a}}^{*}\right\}} \delta\left(L^{*} d t^{*}\right) \\
& =\int_{t_{1}^{*}}^{t^{*},\left\{\boldsymbol{r}_{\boldsymbol{a}}^{*}\right\}} \delta_{t^{*}}\left(L^{*} d t^{*}\right)+\delta_{\left\{\boldsymbol{r}_{\boldsymbol{a}}^{*}\right\}}\left(L^{*} d t^{*}\right)+\delta_{\left\{\frac{d \boldsymbol{r}_{\boldsymbol{a}}^{*}}{d t^{*}}\left(L^{*} d t^{*}\right)+\delta_{t}\left(L^{*} d t^{*}\right)\right.} \\
& =\int_{t_{1}^{*}}^{t^{*},\left\{\boldsymbol{r}_{\boldsymbol{a}}^{*}\right\}}\left\{L^{*} \delta_{t^{*}}\left(d t^{*}\right)+d t^{*} \delta_{t^{*}}\left(L^{*}\right)+\left(\sum_{a} \frac{\partial L^{*}}{\partial \boldsymbol{r}_{\boldsymbol{a}}^{*}} \delta \boldsymbol{r}_{\boldsymbol{a}}^{*}\right) d t^{*}+\left(\sum_{a} \frac{\partial L^{*}}{\left.\left.\partial \frac{d \boldsymbol{r}_{\boldsymbol{a}}^{*}}{d t^{*}} \delta \frac{d \boldsymbol{r}_{\boldsymbol{a}}^{*}}{d t^{*}}\right) d t^{*}\right\}}\right.\right.
\end{aligned}
$$

But $d t^{*}$ is variable:

$$
\begin{gathered}
\delta \frac{d \boldsymbol{r}_{\boldsymbol{a}}^{*}}{d t^{*}}=\delta\left(\frac{d \boldsymbol{r}_{\boldsymbol{a}}^{*}}{d t^{*}}\right)=\frac{\delta\left(d \boldsymbol{r}_{\boldsymbol{a}}^{*}\right)}{d t^{*}}+d \boldsymbol{r}_{\boldsymbol{a}}^{*} \delta\left(\frac{1}{d t^{*}}\right)=\frac{d \delta \boldsymbol{r}_{\boldsymbol{a}}^{*}}{d t^{*}}+d \boldsymbol{r}_{\boldsymbol{a}}^{*}\left(\frac{-\delta d t^{*}}{d t^{* 2}}\right)=\frac{d \delta \boldsymbol{r}_{\boldsymbol{a}}^{*}}{d t^{*}}-d \boldsymbol{r}_{\boldsymbol{a}}^{*}\left(\frac{d \delta t^{*}}{d t^{* 2}}\right) \\
=\frac{d \delta \boldsymbol{r}_{\boldsymbol{a}}^{*}}{d t^{*}}-\frac{d \boldsymbol{r}_{\boldsymbol{a}}^{*}}{d t^{*}}\left(\frac{d \delta t^{*}}{d t^{*}}\right) \\
\delta S\left(\left\{\boldsymbol{r}_{\boldsymbol{a}}^{*}\right\}, t^{*}\right)=\int_{t_{1}^{*}}^{\left.t^{*}, r_{\boldsymbol{a}}^{*}\right\}}\left\{d\left(L^{*} \delta t^{*}\right)-\delta t^{*} d L^{*}+d t^{*} \delta_{t^{*}}\left(L^{*}\right)+\left(\sum_{a} \frac{\partial L^{*}}{\partial \boldsymbol{r}_{\boldsymbol{a}}^{*}} \delta \boldsymbol{r}_{\boldsymbol{a}}^{*}\right) d t^{*}\right. \\
+\left(\sum_{a} \frac{\partial L^{*}}{\left.\left.\partial \frac{d \boldsymbol{r}_{\boldsymbol{a}}^{*}}{d t^{*}}\left(\frac{d \delta \boldsymbol{r}_{\boldsymbol{a}}^{*}}{d d t^{*}}-\frac{d \boldsymbol{r}_{\boldsymbol{a}}^{*}}{d t^{*}}\left(\frac{d \delta t^{*}}{d t^{*}}\right)\right)\right) d t^{*}\right\}}\right.
\end{gathered}
$$




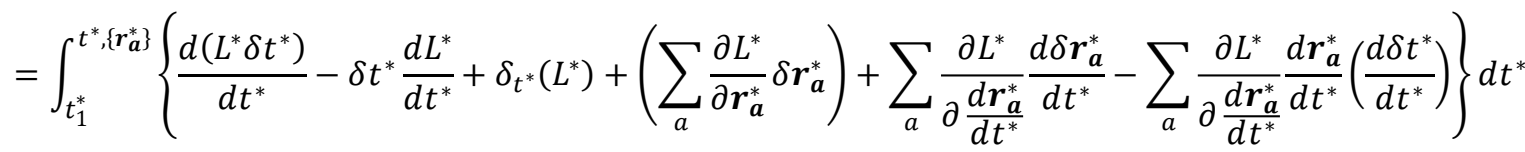

$$
\begin{aligned}
& =\int_{t_{1}^{*}}^{t^{*},\left\{\boldsymbol{r}_{\boldsymbol{a}}^{*}\right\}}\left\{\frac{d\left(L^{*} \delta t^{*}\right)}{d t^{*}}-\delta t^{*} \frac{d L^{*}}{d t^{*}}+\delta_{t^{*}}\left(L^{*}\right)+\left(\sum_{a} \frac{\partial L^{*}}{\partial \boldsymbol{r}_{\boldsymbol{a}}^{*}} \delta \boldsymbol{r}_{\boldsymbol{a}}^{*}\right)\right. \\
& +\left(\frac{d}{d t^{*}}\left(\sum_{a} \frac{\partial L^{*}}{\partial \frac{d \boldsymbol{r}_{\boldsymbol{a}}^{*}}{d t^{*}}} \delta \boldsymbol{r}_{\boldsymbol{a}}^{*}\right)-\sum_{a} \delta \boldsymbol{r}_{\boldsymbol{a}}^{*} \frac{d}{d t^{*}}\left(\frac{\partial L^{*}}{\partial \frac{d \boldsymbol{r}_{\boldsymbol{a}}^{*}}{d t^{*}}}\right)\right)
\end{aligned}
$$

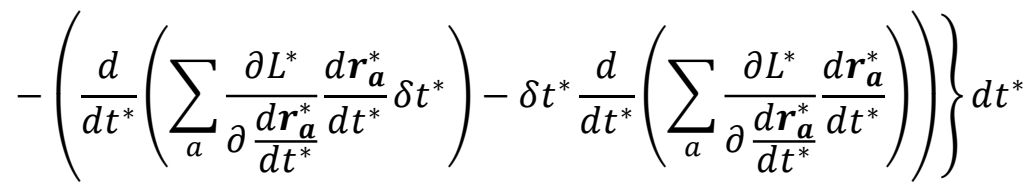

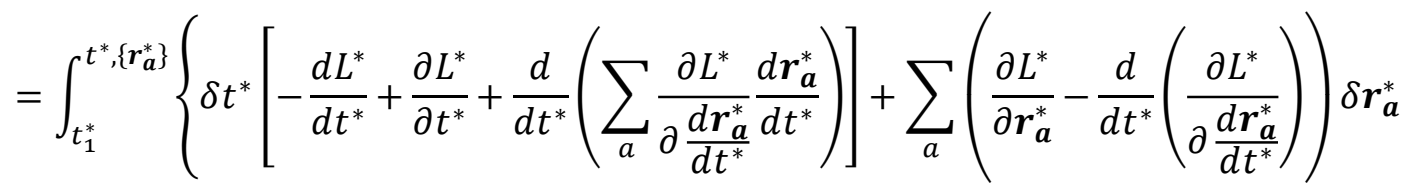

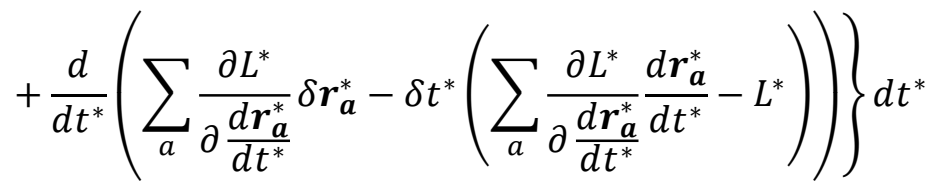

$$
\begin{aligned}
& \text { - }\left(\frac{d}{d t^{*}}\left(\frac{\partial L^{*}}{\partial \frac{d r_{a}^{*}}{d t^{*}} \frac{d r_{a}^{*}}{d t^{*}}}\right)-\frac{d L^{*}}{d t^{*}}=-\frac{\partial L^{*}}{\partial t^{*}}\right)_{\text {for a real trajectory }} \\
& \text { - }\left(\frac{d}{d t^{*}}\left(\frac{\partial L^{*}}{\partial \frac{d r_{a}^{*}}{d t^{*}}}\right)=\frac{\partial L^{*}}{\partial r_{a}^{*}}\right)_{\text {for a real trajectory }}
\end{aligned}
$$

Then we have the following result

$$
\begin{gathered}
=>d S\left(\left\{\boldsymbol{r}_{\boldsymbol{a}}^{*}\right\}, t^{*}\right)=\sum_{a} \frac{\partial S}{\partial \boldsymbol{r}_{\boldsymbol{a}}^{*}} d \boldsymbol{r}_{\boldsymbol{a}}^{*}+\frac{\partial S}{\partial t^{*}} d t^{*} \\
=\sum_{a} \frac{\partial L^{*}}{\partial \frac{d \boldsymbol{r}_{\boldsymbol{a}}^{*}}{d t^{*}}} d \boldsymbol{r}_{\boldsymbol{a}}^{*}-\left(\sum_{a} \frac{\partial L^{*}}{\partial \frac{d \boldsymbol{r}_{\boldsymbol{a}}^{*}}{d t^{*}}} \frac{d \boldsymbol{r}_{\boldsymbol{a}}^{*}}{d t^{*}}-L^{*}\right) d t^{*} \\
=\sum_{a} \boldsymbol{P}_{\boldsymbol{a}}^{*} d \boldsymbol{r}_{\boldsymbol{a}}^{*}-E^{*} d t^{*}
\end{gathered}
$$

Then

- $\boldsymbol{P}_{\boldsymbol{a}}^{*} \equiv \frac{\partial L^{\prime}}{\partial \frac{d r_{a}^{*}}{d t}}=\frac{\partial L^{*}}{\partial \frac{d r_{a}^{*}}{d t^{*}}}=\frac{\partial S}{\partial r_{\boldsymbol{a}}^{*}}$

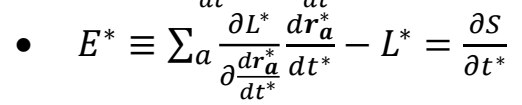

- $L^{*} \equiv \frac{d S}{d t^{*}}\left(\left\{\boldsymbol{r}_{\boldsymbol{a}}^{*}\right\}, t^{*}, t\right)$

- $H^{*}\left(\left\{\boldsymbol{r}_{\boldsymbol{a}}^{*}\right\},\left\{\boldsymbol{P}_{\boldsymbol{a}}^{*}\right\}\right) \equiv \sum_{a} \boldsymbol{P}_{\boldsymbol{a}}^{*} \frac{d \boldsymbol{r}_{\boldsymbol{a}}^{*}}{d t}-L^{*}=\frac{\partial S}{\partial t^{*}}$

- $S\left(\left\{\boldsymbol{r}_{\boldsymbol{a}}^{*}\right\}, t^{*}\right) \equiv\left\{S\left[\left\{\boldsymbol{r}_{\boldsymbol{a}}^{*}\left(t^{*}\right)\right\}, t^{*}\right]\right\}_{\text {real trajectory }}$ 


$$
\left(\frac{E^{*}}{c}\right)^{2}=\left(\frac{E}{c}\right)^{2}-\boldsymbol{P}^{2}
$$

We have the first equation

$$
\frac{1}{c^{2}}\left(\frac{\partial S}{\partial t^{*}}\right)_{t^{*},\left\{\boldsymbol{r}_{a}^{*}\right\}}^{2}=\frac{1}{c^{2}}\left(\frac{\partial S}{\partial t}\right)_{\left\{\boldsymbol{r}_{a}^{*}\right\}, \boldsymbol{R}_{c}, t}^{2}-\left(\frac{\partial S}{\partial \boldsymbol{R}_{\boldsymbol{c}}}\right)_{\left\{\boldsymbol{r}_{a}^{*}, \boldsymbol{R}_{c}, t\right.}^{2}
$$

The expression uses the same quantity $\mathrm{S}$ but expressed as 2 functions of different variables.

We can also express the equation in term of internal position

$$
\begin{aligned}
& E^{*}=\sum_{a} E_{a}^{*} \\
& \text { With }\left(\frac{m_{a} c^{2}}{c}\right)^{2}=\left(\frac{E_{a}^{*}}{c}\right)^{2}-\boldsymbol{P}_{\boldsymbol{a}}^{* 2} \\
& =>E_{a}^{*}=\sqrt{\left(m_{a} c^{2}\right)^{2}+c^{2} \boldsymbol{P}_{\boldsymbol{a}}^{* 2}} \\
& =>E^{*}=\sum_{a} \sqrt{\left(m_{a} c^{2}\right)^{2}+c^{2} \boldsymbol{P}_{\boldsymbol{a}}^{* 2}} \\
& =>\sqrt{\left(\sum_{a} \sqrt{\left(\frac{m_{a} c^{2}}{c}\right)^{2}+\left(\frac{\partial S}{\partial \boldsymbol{r}_{\boldsymbol{a}}^{*}}\right)_{t^{*},\left\{\boldsymbol{r}_{\boldsymbol{a}}^{*}\right\}}^{2}}\right)^{2}=\frac{1}{c^{2}}\left(\frac{\partial S}{\partial t}\right)_{\left\{\boldsymbol{r}_{\boldsymbol{a}}^{*}\right\}, \boldsymbol{R}_{\boldsymbol{c}}, t}^{2}-\left(\frac{\partial S}{\partial \boldsymbol{R}_{\boldsymbol{c}}}\right)_{\left\{\boldsymbol{r}_{\boldsymbol{a}}^{*}\right\} \boldsymbol{R}_{\boldsymbol{c}}, t}^{2}}
\end{aligned}
$$

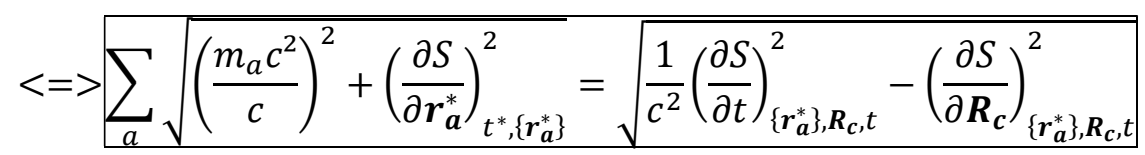

This equation is pretty complicated. To develop again the analysis, a quantum version (à la Schrödinger) using the action as the phase of a wave function would be interesting to obtain (with the internal degree of freedom and the center of mass as variables). Here we see that is seems not very straightforward (or not possible?). 


\subsection{Annex calculation}

We want to draw the $\mathrm{K}^{*}$ axis seen by $\mathrm{K}$, that is to say the different axis in function of the $\mathrm{x}$ axis.

$$
\left\{\begin{array}{l}
c . t-c . t_{i}=\gamma_{t_{i}} \cdot\left(\mathrm{c}\left(t_{K_{i}^{*}}-t_{C\left(t_{i}\right)}^{*}\right)+\beta_{t_{i}} \cdot x_{K_{i}^{*}}\right) \\
x-x_{c}\left(t_{i}\right)=\gamma_{t_{i}} \cdot\left(x_{K_{i}^{*}}+\beta_{t_{i}} \cdot\left(t_{K_{i}^{*}}-t_{C\left(t_{i}\right)}^{*}\right)\right)
\end{array}\right.
$$

○ In K, the equation of a static point in $\mathrm{K}^{*}\left(x_{K_{i}^{*}}=\right.$ cte $)$ in function of $\mathrm{x}$, that is to say c. $t_{\left(x_{K_{i}^{*}}=c t e\right)}(x)$ is

$$
\begin{aligned}
& \text { c. } t-c . t_{i}=\gamma_{t_{i}} \cdot\left(c\left(t_{K_{i}^{*}}-t_{C\left(t_{i}\right)}^{*}\right)+\beta_{t_{i}} \cdot x_{K_{i}^{*}}\right)=\gamma_{t_{i}} \cdot\left(\frac{x-x_{c}\left(t_{i}\right)}{\beta_{t_{i}} \cdot \gamma_{t_{i}}}-\frac{x_{K_{i}^{*}}}{\beta_{t_{i}}}+\beta_{t_{i}} \cdot x_{K_{i}^{*}}\right) \\
& =\frac{x-x_{c}\left(t_{i}\right)}{\beta_{t_{i}}}-x_{K_{i}^{*}} \cdot \gamma_{t_{i}} \frac{1}{\beta_{t_{i}}}\left(1-\beta_{t_{i}}{ }^{2} \cdot\right)=\frac{x-x_{c}\left(t_{i}\right)}{\beta_{t_{i}}}-\frac{x_{K_{i}^{*}}}{\gamma_{t_{i}} \cdot \beta_{t_{i}}} \\
& \text { c. } t=c . t_{i}+\frac{x-x_{c}\left(t_{i}\right)}{\beta_{t_{i}}}-\frac{x_{K_{i}^{*}}}{\gamma_{t_{i}} \cdot \beta_{t_{i}}} \\
& =>c \cdot t_{\left(x_{K_{i}^{*}}^{*}=K\right)}(x)=c \cdot t_{i}+\frac{x-x_{c}\left(t_{i}\right)}{\beta_{t_{i}}}-\frac{K}{\gamma_{t_{i}} \cdot \beta_{t_{i}}} \text { at time } \mathrm{t}=t_{i}
\end{aligned}
$$

So the equation of $x_{K_{i}^{*}=0}$ is $\quad$ c. $t_{\left(x_{K_{i}^{*}}=0\right)}(x)=c \cdot t_{i}+\frac{x-x_{c}\left(t_{i}\right)}{\beta_{t_{i}}}$ at time $\mathrm{t}=t_{i}$

Between $x_{c}\left(t_{1}\right)$ and $x_{c}\left(t_{2}\right)$, the variation is at should:

$c . t_{\left(x_{K_{1}^{*}}=0\right)}\left(x_{c}\left(t_{2}\right)\right)-c \cdot t_{\left(x_{K_{i}^{*}}^{*}=0\right)}\left(x_{c}\left(t_{1}\right)\right)=\frac{x_{c}\left(t_{2}\right)-x_{c}\left(t_{1}\right)}{\beta_{t_{1}}}=\frac{V_{c}\left(t_{1}\right) \cdot\left(t_{2}-t_{1}\right)}{\beta_{t_{1}}}=c \cdot\left(t_{2}-t_{1}\right)$

- In K, the equation of $\left(\mathrm{t}^{*}=\mathrm{cte}\right)$ in function of $\mathrm{x}$, that is to say $c . t_{\left(t^{*}=c t e\right)}(x)$ is

$$
\begin{aligned}
& \left\{\begin{array}{l}
c . t-c . t_{i}=\gamma_{t_{i}} \cdot\left(\mathrm{c}\left(t_{K_{i}^{*}}-t_{C\left(t_{i}\right)}^{*}\right)+\beta_{t_{i}} \cdot x_{K_{i}^{*}}\right) \\
x-x_{c}\left(t_{i}\right)=\gamma_{t_{i}} \cdot\left(x_{K_{i}^{*}}+\beta_{t_{i}} \cdot\left(t_{K_{i}^{*}}-t_{C\left(t_{i}\right)}^{*}\right)\right)
\end{array}\right. \\
& \text { c. } t-c . t_{i}=\gamma_{t_{i}} \cdot\left(\mathrm{c}\left(t_{K_{i}^{*}}-t_{C\left(t_{i}\right)}^{*}\right)+\beta_{t_{i}} \cdot x_{K_{i}^{*}}\right) \\
& =\gamma_{t_{i}} \cdot\left(\mathrm{c}\left(t_{K_{i}^{*}}-t_{C\left(t_{i}\right)}^{*}\right)+\beta_{t_{i} \cdot}\left(\frac{x-x_{c}\left(t_{i}\right)}{\gamma_{t_{i}}}-\beta_{t_{i}} \cdot \mathrm{c}\left(t_{K_{i}^{*}}-t_{C\left(t_{i}\right)}^{*}\right)\right)\right) \\
& \text { c.t }-c . t_{i}=\gamma_{t_{i}} \cdot\left(\mathrm{c}\left(t_{K_{i}^{*}}-t_{C\left(t_{i}\right)}^{*}\right)+\beta_{t_{i}} \cdot \frac{x-x_{c}\left(t_{i}\right)}{\gamma_{t_{i}}}-\beta_{t_{i}}{ }^{2} \cdot \mathrm{c}\left(t_{K_{i}^{*}}-t_{C\left(t_{i}\right)}^{*}\right)\right) \\
& =\gamma_{t_{i}}\left(\left(1-\beta_{t_{i}}^{2}\right) \mathrm{c}\left(t_{K_{i}^{*}}-t_{C\left(t_{i}\right)}^{*}\right)+\beta_{t_{i}} \frac{x-x_{c}\left(t_{i}\right)}{\gamma_{t_{i}}}\right)=\frac{\mathrm{c}\left(t_{K_{i}^{*}}-t_{C\left(t_{i}\right)}^{*}\right)}{\gamma_{t_{i}}}+\beta_{t_{i^{*}}}\left(x-x_{c}\left(t_{i}\right)\right) \\
& \text { c. } t=c . t_{i}+\beta_{t_{i}} \cdot\left(x-x_{c}\left(t_{i}\right)\right)+\frac{\mathrm{c}\left(t_{K_{i}^{*}}-t_{C\left(t_{i}\right)}^{*}\right)}{\gamma_{t_{i}}}
\end{aligned}
$$




$$
\Rightarrow c \cdot t_{\left(c t_{K_{i}^{*}}=K\right)}(x)=c \cdot t_{i}+\beta_{t_{i}} \cdot\left(x-x_{c}\left(t_{i}\right)\right)+\frac{c\left(K-t_{C\left(t_{i}\right)}^{*}\right)}{\gamma_{t_{i}}}
$$

And in particular

$$
c . t_{\left(c t_{K_{i}^{*}}=t_{C\left(t_{i}\right)}^{*}\right)}(x)=c \cdot t_{i}+\beta_{t_{i} \cdot}\left(x-x_{c}\left(t_{i}\right)\right)
$$




\section{References}

[1] L. Landau and E. Lifshitz, "Théorie des Champs", section 8, 29, 5th edition, Mir-Ellipses, Moscow (1994)

[2] L. Landau and E. Lifshitz, “Mécanique”, section 44, 5th edition, Mir-Ellipses, Moscow (1994)

[3] Michel Janssen, Matthew Mecklenburg, "Electromagnetic Models of the Electron and the Transition from Classical to Relativistic Mechanics ", in V. F. Hendricks, et.al., Interactions: Mathematics, Physics and Philosophy, Dordrecht, Springer, 65-134 (2007)

[4] Wang, Changbiao, "Von Laue's Theorem and Its Applications", in Canadian Journal of Physics, vol. 93, issue $12,1470-1476$ (2015)

[5] Yvan Simon, « Relativité restreinte », pp130-155.VUIBERT, Paris (2004)

[5’] José Philippe Pérez, "Relativité et invariance », Fondements et applications, $2^{\text {ème }}$ édition, Dunod, Paris , Paris $(1999,2005)$

[6] Dominique Lambert, "Le Triomphe d'un chanoine " , in "Lemaître, Le père du Big Bang - Les génies de la science $\mathrm{N}^{\circ} 30$ ", (Février 2007)

[7] Eugene Hecht, "How Einstein confirmed $E_{0}=m^{22 ", ~ i n ~ A m e r i c a n ~ J o u r n a l ~ o f ~ P h y s i c s ~ 79, ~} 591$ (2011)

[8] Einstein, " AKosmologische Betrachtungen zur allgemeinen Relativitätstheorie». Sitz. König. Preuss. Akad. 142-152. Available in English translation as"Cosmological considerations on the general theory of Relativity", in (Lorentz et al.1952): 175-188.(1917)

[9] Thibault Damour. Poincaré, the Dynamics of the Electron, and Relativity. Comptes Rendus Physique, 2017, 18, pp.551-562. 〈10.1016/i.crhy.2017.10.006). 〈hal-01669824〉

[10] Hans C. Ohanian, Einstein's $E=m c^{2}$ mistakes https://arxiv.org/ftp/arxiv/papers/0805/0805.1400.pdf

[11] Hans $C$. Ohanian, Klein's theorem and the proof of $E_{0}=m c^{2}$ American Journal of Physics 80, 1067 (2012); https://doi.org/10.1119/1.4748812

[12] L B Okun, The Einstein formula: $\mathrm{E}_{0}=\mathrm{mc}^{2} .:$ "Isn't the Lord laughing?" https://doi.org/10.1142/97898128141280029

[13] Jean Pierre-Luminet, Le destin de I'univers I, Chapitre VIII, Les gravasters, Folio Essais

[14] Albert Einstein, Oeuvres choisies, Relativités I, Relativité Restreinte et Générale, Sources du savoir Seuil,CNRS

[15] Albert Einstein “Ist die Tr̈ agheit eines K'orpers von seinemEnergieinhalt abh"angig?"Ann. Phys. (Leipzig) 18 [323] 639-641 (1905) [Translated into English: "Does the inertia of a body depend on its energy content?", in CPAETVol. 2, doc. 24 
[16] Albert Einstein A "Das Prinzip von der Erhaltung der Schwerpunktsbewegung und dieTr̈ agheit der Energie" Ann. Phys. (Leipzig) 20 [325] 627 - 633 (1906) [Translatedinto English: "The principle of conservation of motion of the center of gravity and the inertia of energy", in CPAET Vol. 2, doc. 35

[17] Albert Einstein "Uber das Relativit atsprinzip und die aus demselben gezogene Fol-gerungen" Jahrbuch Radioaktivit" at Elektron. 4411 - 462 (1907) [Translated into English: "On the relativity principle and the conclusions drawn from it", inCPAET Vol. 2, doc. 47;

[18] Albert Einstein "Manuscript on the special theory of relativity (1912 - 1914)", inCPAET Vol. 4, doc. 1

[19] Albert Einstein Vier Vorlesungen "'Uber Relativit" atstheorie, Gehalten im Mai, 1921,an der Universit at Princeton" (Braunschweig, Germany: Friedrich Vieweg undSohn, 1922) [Translated into English: "Four lectures on thetheory of relativity, held at Princeton University in May 1921, in CPAET Vol. 7, doc. 71

[20] Albert Einstein, "Elementary derivation of the equivalence of mass and energy" Bull.Am. Math. Soc. $41223-230$ (1935)

[21] Albert Einstein, "Elementary derivation of the equivalence of mass and energy" Tech-nion J. 516 (1946)

[22] Einstein A, Die Grundlage der allgemeinen Relativitätstheorie. (The foundation of the General Theory of Relativity).Annalen derPhysik, 49, 769-822 (also published separately as Leipzig: Teubner) [CPAE 6,283-339.]

[23] Henri Poincaré, Sur la dynamique de l'électron, 1906, Rendiconti del Circolo matematico di Palermo 21: 129-176, Adunanza del 23 luglio 1905. Stampato il 14-16 dicembre 1905

[24] Christian Bracco,Poincaré et la Nouvelle Mécanique : de la dynamique de l'électron (1905) aux Dernières pensées (1912) https://www.canalu.tv/video/cerimes/poincare_et_la_nouvelle_mecanique_de_la_dynamique_de_I_electron_1905_au x_dernieres_pensees_1912.10002

[25] Christian Bracco, Jean-Pierre Provost, De l'électromagnétisme à la mécanique : Le rôle de l'action dans le Mémoire de Poincaré de 1905, Dans Revue d'histoire des sciences 2009/2 (Tome 62), pages 457 à 493

[26] Cormac O'Raifeartaigh, Historical and Philosophical Aspects of the Einstein World, https://arxiv.org/abs/1906.08678 